\title{
Do I Get a Say In This? \\ Environmental Sound Composition, the Phonograph and Intentionality
}

\author{
By
}

Thomas Edward Voyce

\begin{abstract}
A thesis
submitted to the Victoria University of Wellington in fulfilment of the requirements for the degree of Doctor of Philosophy
\end{abstract}




\section{Abstract}

Environmental sound composition, a term I employ to describe all forms of electroacoustic works in which the core materials are abstracted from real environments through technology, has been practiced in a variety of forms for more than 50 years. A tension exists between environmental sound composition and western art music, one that continues to make this marriage uncomfortable. In short, the use of mimetic materials in environmental sound composition does not fit the prescriptions of formalism, an ideology that electroacoustic composition inherited from western art music. Though attempts have been made to lessen this tension (Emmerson, 1986 and Smalley, 1996), an underlying anxiety persists in environmental sound composition, as the twin legacy of Pierre Schaeffer's ideas concerning musique concrète and the concerns of acoustic ecology, a movement championed by soundscape composers (Westerkamp, 2002), continues to influence the genre.

Recently there has emerged an increasing resistance to the didactic ideology of soundscape theory in particular, as exemplified by Lopez (1997), Ingold (2007) and Kelman (2010). However, soundscape theory continues to influence the production of environmental sound composition, as composers seek to align themselves with such concerns, or place themselves in opposition to them. In my view, the tension between formalism and mimesis has resulted in a widespread fixation on poietic intent in environmental sound composition. As a result, composers have tried to dictate how their works should be heard, while ignoring the complexity of listener response. While a number of fresh perspectives have arisen in recent years looking at environmental sound composition methodology and the role of esthetic analysis in such works, including Voegelin (2010) and Lane and Carlyle (2013), a rigorous investigation into the roles of intentionality, technology and hermeneutic analysis in the production and reception of environmental sound composition remains absent. 
My thesis explores the nature of the phonograph (an audio recording) and phonography (the act of recording) in broad terms, and then with specific attention to environmental sound composition. Various recording genres and phonograph types associated with these genres are identified, while the attitudes of composers towards technology and the ontological nature of their works are investigated. This approach is applied in making a critical assessment of environmental sound composition, exposing the specificity of the rift between poietic intention and esthetic reception. I argue for a hermeneutic evaluation of the phonograph on similar terms as those set out by Roland Barthes in Camera Lucida (1981). In examining the temporal dimensions of the phonograph, along with its formal and affective traits, my research aims to elevate the phonograph from the role of a passive bearer of composer intentionality, to that of a primary contributor to the listening experience. With this aim in mind, I present a portfolio of creative works as a second volume to this thesis, born of the ideas discussed herein, which explore the nature of the phonograph, its temporalities, the site specific aspects of phonography and compositional intervention with the phonograph. I will refer to my works throughout this thesis, detailing how I have incorporated my theoretical concerns into my compositional practice, especially in chapter four, five and six. 


\section{Acknowledgements}

As so many $\mathrm{PhD}$ candidates are quick to recognise, focused research can only be made possible through the generous support of many people, a number of whom may be unaware of the impact their contribution has made. This particular research project has been supported, directly and indirectly, by more people than I can name, but I would like to take this opportunity to thank the ones whose support has allowed this research to happen at all.

To begin, I would like to thank my supervisor, Dr. Dugal McKinnon, who has guided me at postgraduate level for almost 10 years. Without his attention, ear, and inexhaustible knowledge for all things sound art, this project simply would not have happened. To my fiancée Jina, whose patience, encouragement and belief in my ability to multitask has known no bounds, thank you. To my parents, whose cries of "is it finished yet?" have spurred me on, especially in these last six months, thank you. To my mother-in-law Me Li, who allowed me to finish this thesis by looking after my daughter during the final months, thank you. I would also like to thank my friends and colleagues at Rhombus Productions Limited, especially Koa Williams and Simon Rycroft, for their ongoing support throughout this $\mathrm{PhD}$, as time dedicated to this research has meant time away from our collaborative projects.

I am also greatly indebted to the wider community known as the New Zealand School of Music, especially the composition administration staff (Fiona Steedman in particular), who have assisted me along the way both as a postgraduate student and as a teacher. To my students, thank you for your interest in my research, it has been very encouraging to know that others (some of you anyway) are interested in these ideas as much as I am.

Finally, I would like to dedicate this thesis to my daughter, Maya. She has watched her father disappear to work after dinner for many years, and the 
conclusion of this project means a few less nights away from home, and a bit more time spent on her homework, not mine. 


\section{Contents}

\section{Volume I}

ABSTRACT

CHAPTER 1:

FROM THE PHONOGRAPH-AS-DOCUMENT TO THE PHONOGRAPH-AS-WORK

CHAPTER 2:

THE PHONOGRAPH-AS-WORK

CHAPTER 3:

92

INTENTIONALITY, ENVIRONMENTAL LISTENING AND FIELD RECORDING

CHAPTER 4:

SOUNDSCAPE COMPOSITION, SEMIOLOGY, INTENTION AND RECEPTION

CHAPTER 5:

AURAL AND VisUAL EXPERIENCE: PHONOGRAPHY, FILM AND PHOTOGRAPHY 
CHAPTER 6:

STUDIUM, PUNCTUM, ANAMNESIS: TOWARD AN ONTOLOGY OF THE PHONOGRAPH

\section{Volume II}

PORTFOLIO OF WORKS 


\section{Introduction}

Environmental sound composition is a genre of acousmatic music that has captured my imagination for more than 20 years, though it was not until I reached university that I discovered it had a name, and that it was part of broader genre of music. As a teenager, I would record just about anything with my father's Sony Dictaphone: conversations, crowds at sports games, my friends and I drumming on our knees in the empty WWII gun emplacements that overlook Wellington Harbour. I have boxes of such recordings, some on cassette, some on DAT, a few on minidisc. I now have hard drives full of recordings. I have taken my recorder with me on many international excursions. My desire to seek out new environments to record has not waned at all and is something I have practiced with enthusiasm for many years, though initially I did not give much consideration as to why. It has always seemed a natural activity, albeit an uncommon one. I simply enjoyed the process of recording and playing back events.

When I was 17, I went to university to study composition. Sometime over the next year, I became aware of the term soundscape composition. I immediately thought I had discovered something significant - this was a genre that related directly to the kind of recordings I had been making for a number of years. I felt that soundscape composition provided a framework within which I could legitimately and comfortably extend the activity I had long been practicing. Or was it? Acoustic ecology seemed to be a central concern of the genre, and while I could empathise with such concerns, it occurred to me that my engagement with environmental sound was not born of a desire to fix it, to teach others about it, or to connect with others through it on any level whatsoever. My engagement with the environment was deeply personal, and my use of field recording in acousmatic works equally so.

Nevertheless, I persevered with some electroacoustic composition papers. Where digital processing was concerned, I was as much fascinated by the 
potential for processed field recordings as I was by the recordings themselves, and my electroacoustic works during this time reflected that. I would layer processed recordings with unprocessed ones to create interesting hybrid environments that were part 'real' and part fantasy. I would sometimes move from processed recordings to unprocessed recordings within a piece to explore narrative ideas, but mostly I was just content to hear environmental sound recorded, as I always had. The grades I received for my work were not fantastic. Not only did my works not fit with the concerns of soundscape composition, but apparently they did not fit with the formal concerns of electroacoustic composition either. I found myself caught between two theoretical perspectives without understanding how I got to be in that place, or why I felt a pressure to accommodate the concerns of one or other ideology. I left university without becoming a soundscape composer, or an electroacoustic composer. I also left feeling bemused as to why my experience of the combination of technology and environmental sound, a mix with which I had always felt comfortable, had failed to find a home in my university music department. Still, I kept recording.

My early experience at university highlights a series of issues that form the fundamental concerns of this thesis. In preparation for this research I asked myself a number of questions: what are the aspects of western art music and soundscape composition ideologies that have had such enduring influence over environmental sound composition? What exactly is soundscape composition, and how do its composers understand recording technology to operate in regard to their concerns? What are the differences between situated experience and nonsite experience where environments and recordings of them are concerned? What exactly is a recording - the phonograph - and are there different types of these? And most importantly, why is there such a division between what is intended by a composer and what is experienced by a listener where environmental sound composition is concerned, and is there a way to address this division that recognises the experience of the listener? This particular question, born of my earlier experiences at university, has given rise to another significant question: is there another form of mediatised experience that can 
assist in a listener-focused evaluation of environmental sound composition and the phonograph?

I discovered that a great deal of these questions were yet unanswered. Though there has been a renewed interest in environmental sound composition in recent years, witnessed not least of all in the increasing number of compositions produced and the flurry of books and papers written on the subject (many of which are cited below), no one has adequately assessed the ideas I feel directly inform environmental sound composition, particularly the notions of composer and listener intentionality, where environmental materials are involved, and the divide between intention and reception at the level of hermeneutic analysis. I also feel the specificity of the phonograph, its core features and how it can be appreciated, beyond its assumed role as a delivery format for a composer's compositional concerns, remains largely unexamined. This thesis attempts to pull these threads together, as I will now detail.

Chapter one adopts a broad perspective of the phonograph, as I look to distinguish various phonograph types. I engage primarily with the writings of Edison (1878/2012), Katz (2004), Ashby (2010), Goehr (1994), Gould (1967/2004) and Stravinsky (1936/2012), while extending the ideas of Dellaira (1995) in particular. From the outset, I instate two fundamental ontological features of the phonograph, understood as 'documentary' and 'abstraction' respectively, while setting up a continuum of phonograph types: from the phonograph-as-document to the phonograph-as-work. I look at notions of what 'the work' is, especially with regard to concepts prevalent in classical music. I then look at how subtle changes in production methodologies, particularly the splice, alter the phonograph at an ontological level. Travelling along the continuum towards the phonograph-as-work, I examine how various recording techniques can produce a series of temporal relationships between the listener and the event(s) recorded, exploring the recording practices of classical music, punk, pop, reggae and hip hop in particular. The value of this chapter is to both lay the groundwork for future discussions of the phonograph and its features in subsequent chapters, and also to reveal commonalities between various 
recording genres. In revealing such similarities between recording genres, a dialogue regarding the phonograph opens up between genres with seemingly opposed aesthetics as far as technology is concerned. These connections are made throughout the thesis.

Chapter two explores the phonograph-as-work within the genre of electroacoustic composition. Regarding the relationship of the phonograph and the work within electroacoustic music, I examine the writings of Eimert (1957), Stockhausen (Tannenbaum, 1987), Schaeffer $(1966,2012)$ and Chion (2009). I then look to Ferrari (Drott, 2009; Warburton, 1998), for a discussion of environmental sound within the context of musique concrète, and follow with a discussion of 'mimesis', that is, real word sounds and references, in electroacoustic composition (Emmerson, 1986). I conclude the chapter with a discussion of Emmerson, and the way in which the phonograph-as-work functions within his influential 'language grid' (1986, p. 17-39). This chapter provides a means to explore not only attitudes towards the phonograph within electroacoustic composition, but also a means to discuss the role of mimesis in electroacoustic composition. This particular discussion has significance to both the discussion of environmental sound composition in chapter four, and also the discussion of intentionality, the subject of chapter three, as the notions of formalism and how to deal with mimetic materials are revealed to influence the intentional perspectives of several important figures, especially Cage and Schaeffer.

Chapter three explores the notion of intentionality, as a way to examine the similarities and differences between listening practices, especially in-situ listening and phonographic listening. Such an examination reveals the ways in which environmental sound composers, though they are not alone in this, believe the phonograph functions in relation to their poietic intentions (a discussion of which is extended in chapter four). Beginning in this chapter, I also begin to discuss the ideas presented in relation to my own compositions (those found in Volume II). This chapter engages with the idea of Husserlian intentionality through the writings of McIntyre and Smith (1989) and Russell (2006). It also 
explores the contribution of Russolo (1913), Ruttmann (1930) and Cage among others, in the development of the 'musicalisation' of the sonic environment within western art music (Kahn, 1999). Further to this, a discussion of situated listening experience is made through the writings of Fisher (1998), Schaeffer (2012), Voegelin (2010) and Ingold (2007). Particular attention is paid to the differences between situated listening and phonographic listening. This is examined through the observations of Toop (2004) and Ihde (2007), with commentary about field recording practices and what the phonograph affords a listener from Winderen and Kubisch (Carlyle and Lane, 2013). The chapter concludes with a discussion of the phonograph's core features (documentary and abstraction) as they are thought to function in the practice of field recording. In doing so I look to Schafer's notion of 'schizophonia' (1977) and Feld's related notion of 'schismogenesis' (1994), with further commentary from Lopez (1997). This chapter promotes the notion of listener intentionality; that is, a phenomenological understanding of listening that distinguishes the listener's perspective from that of the composer, as a crucial factor in field recording practices. I discuss how intentionality operates in the shaping of composers' perspectives surrounding the nature of the phonograph and what it is thought to offer a listener.

Chapter four extends the idea of intentionality into the world of soundscape composition, looking specifically at how it defines soundscape composition (Truax, 2001) and acoustic ecology (Schafer, 1977) as well as the marriage of the two (Westerkamp, 2002). I then assess criticisms of the genre, including those made by Cusack (2000), Kelman (2010), and Ingold (2007). I also revisit the notions of schizophonia and schismogenesis, extending them into the discussion of soundscape composition, with particular emphasis on the paradox schizophonia presents to the educational aspirations of the genre. Having established that soundscape composition does indeed aspire to educate listeners through composition, I turn to semiology through the ideas of Nattiez (1990) and Smalley (1996), to examine the way in which soundscape composition and the phonograph are thought to operate in the transmission of a work's meaning to a listener. I then explore the ways in which the pedagogical dimensions of 
soundscape composition might operate, primarily through the writings of Rancière (1991, 2009). Here I also discuss the role dramaturgy (Weale, 2005), that is, the contextual information that accompanies an electroacoustic piece, plays in intention and reception. Having explored the poietic processes of soundscape composition from intentional, semiological and educational perspectives, I conclude that soundscape composers believe the phonograph offers a listener a great deal more than it can, and that listener intentionality struggles to meet the demands of composer intention.

In chapter five, I look to promote the listener (not the composer) to the position of fundamental determiner of the phonograph's affective power and in doing so I explore other mediatised experiences, notably photography and film, for points of comparison. I explore aspects of the nominalist work (Goehr, 1992) within a brief discussion of photography before moving on to a discussion of film, and particularly the concept of the audiovisual contract (Chion 1994), as well as notions of temporality, presence and the temporal span of aural awareness (Ihde, 2007). I then pursue a comparison of silent film and the phonograph, followed by discussions of 2D and 3D cinema in comparison to phonography, recounting examples by Wenders (2015) and Herzog (2010), and the various ways in which visual and aural experience differ when attending to these formats. I also explore the role of aural architecture (Blesser and Salter, 2007) in aural experience, and how this contributes to a sense of presence and reality in phonographic replay. With a final note from Barthes (1981), I examine the role of protensity in phonography and film, that is, the ability for past events to reach forward to the present, and how these two mediums differ as a result of differences between aural and visual experience, concluding that photography provides the phonograph with the most useful points of comparison. This chapter has a number of functions. It explores the notion of temporality in aural experience as developed throughout the thesis, and provides a context for the final chapter, which involves a hermeneutic analysis of the phonograph in Barthesian terms. 
In chapter six, I explore ideas laid out in Barthes' Camera Lucida (1981), with critique from Rancière (2013), and apply these ideas to the phonograph. In particular, I explore the notions of adventure, studium and punctum, and how these might apply to the appreciation of the phonograph. I also outline how soundscape composition fails to elicit the desired response from listeners within the context of this hermeneutic model. I extend Barthes' ideas to include the idea of technological puncta, and the role of sonic signatures in such an appreciation. I also detail additional features that the phonograph presents, particularly the phenomenon of anamnesis (Augoyard and Torgue, 2006) and its role in phonographic experience. This chapter contains many examples from my own experiences of listening to phonographs, which constitutes a personal account of listener response. In doing so, I reveal the nature of temporality in listener experience, especially the perception of the present, the perception of the past event, and the memories of others and events that the phonograph evokes. This final chapter serves to highlight the role of the listener in phonographic appreciation, by emphasising the very personal nature of the individual's intentional perspective.

The second volume of this thesis constitutes an appraisal of the works found in my portfolio of compositions. This appraisal, perhaps best understood as extended programme notes to accompany the listening experience, is designed to provide insight into my compositional processes, exposing relationships between my practiced-based research and the ideas examined in this thesis. The portfolio of works was indeed developed alongside this research, and as such, it is important for the reader/listener to gain a sense of how the creative component of this submission relates to my critical and theoretical research. I am well aware that in providing the reader/listener access to my poietic intentions, I am in some ways detracting from an uninhibited reader response, the very kind of engagement this thesis argues for. However, it is not my intention to dictate how a reader/listener should engage with my work, rather I hope that by providing some insight to my methodology, they might be able to determine the extent to which my compositions address the fundamental concerns of this thesis, without detracting from their personal engagement with 
the phonographs they audition. With this in mind, it is my strong preference that the compositions contained within this second volume are auditioned before the thesis and the accompanying programme notes.

Returning now to my first experiences at university as a young environmental sound composer, this research goes a long way towards accounting for the feeling of bemusement I experienced. It would appear that many composers, environmental sound composers and otherwise, struggle to accept that composer intentionality is not the primary focus of listener engagement with the phonograph, and attempting to promote composer intentionality above all else can have undesired results. I argue that given the wealth of opposition to soundscape composition (Lopez, Kelman, Cusack, Toop et al.) and the attempts by some composers to gain a greater audience for electroacoustic music (Landy, Weale), such a focus on poietic intent may be turning people away from works they might otherwise enjoy. This thesis begins with an appraisal of different phonograph types. By the end, it is my hope that these distinctions, coupled with a greater understanding of what the phonograph can and cannot offer, results in an appreciation of not only the specificity of the phonograph, but also the complex and deeply engaging experience of phonographic listening. Given all this my primary question is a simple but difficult one: do we really need to tell people how to listen? 


\section{1}

From the Phonograph-as-Document to the Phonograph-as-Work

\section{Use of the term 'phonograph'}

As first mentioned in the abstract of this thesis, I have elected to employ the term 'phonograph' to describe an audio recording, as opposed to the more commonly used term 'phonogram'. While the suffix - graph (derived from Greek, meaning "drawing" or "writing") is more commonly used to describe a device, and the suffix -gram ("drawn" or "written") is used to describe the product of such a device, this is not always the case and the photograph is a notable example. Though my use of the term 'phonograph' has some potential for confusion (as I will discuss shortly, Edison's famous recording and playback device from 1877 was known as the phonograph), I nevertheless prefer the suffix -graph overgram, in the context of this thesis, for two reasons. The first is that it aligns my terminology with the related practice of photography. Just as a photograph is the product of photography, a phonograph is here considered a product of phonography (the act of recording). A comparison of phonography with photography is made in chapter five and in greater detail throughout chapter six, and as such, the alignment of terminology between these practices assists this comparison.

The second reason for using 'phonograph' is that the suffix -gram has, to my mind, an air of reduction; reifying a recording as a static object. A 'phonogram' is distinct from its "writing" and also its playback. In other words, it is an 
unactualised (non-aural) entity. As such, we might consider a phonogram to be a 12 " record, sitting on a shelf, or a digital audio file, stored in a hard drive. In my view, an audio recording implicitly implies its production in a way that other grams do not. A telegram, for example, does not implicitly reference the technology that produced it (the telegraph), in part because an intermediate stage of decoding is required to produce it. Once the electronic signals of the telegraph are decoded, a telegram is much like any other written word (a letter, a book); it stands alone from its production. Where listener experience is concerned, an audio recording cannot be separated from its production, nor can it be separated from its reproduction. In this way an audio recording has three unique aspects that the term 'phonogram' does not adequately account for: the production of the recording, the unactualised recording stored in a medium, and its reproduction through playback technology. For this reason, and in my view the term 'phonograph', with its implicit reference to the writing (and re-writing) of sound waves, better captures the essence of phonographic experience.

\section{'Documentary' and the phonograph}

The power of audio technology to capture and reproduce past events has given rise to a pervasive reading of such technology: one in which the phonograph is understood as a type of 'document'. In many respects, the power of the phonograph (the device) to record the past is the most immediate and easily understood usage, as Edison himself identified upon hearing the first reproductions of his invention in 1877. Writing in the North American Review the following year, Edison confessed he was 'in a somewhat chaotic condition of mind' regarding the possibilities of his invention (Edison, 1878/2012, p. 29), though it appears that the majority of his proposed uses for his phonograph were underscored by this key observation. In the same publication, Edison detailed some of the applications he felt his phonograph could excel at:

1) Dictation

2) Books (readings of books already written for the blind and for those "whose hands may be otherwise employed") 
3) Education (specifically, elocution)

4) Music

5) Family Record (in Edison's description, not unlike family photographic portraiture)

6) Phonographic Books (literary works specifically for the phonograph)

7) Musical Boxes, Toys, etc., (Edison notes that should the audio quality of his phonograph never improve, at the least his phonograph can give the music box "the voice and words of the human songstress")

8) Toys (dolls, animals and locomotives "may be supplied with their natural and characteristic sounds")

9) Clocks (with a human voice and time-related messages)

10) Advertising

11) Speech and Other Utterances (Edison expresses the desire to preserve presidential speeches for playback on holidays)

12) Recording Telephone Conversations (1878/2012, p. 34-36)

As evidenced in his list, he felt his phonograph could be used in either the fields of dictation and education, or for products with a more commercially driven focus, as demonstrated by his ideas for toys, clocks and books. It seems that Edison immediately identified that the primary power of his invention lay in its ability to reproduce sound waves from the past in the present, which amounts to a technical appreciation of the apparatus (hardly surprising given he invented it). Jonathan Sterne (2003) uses the term 'tympanic' to describe the technical function of diaphragms in sound reproduction, further noting 'it is still impossible to think of a configuration of technologies that makes sense as sound reproduction without either microphones or speakers' (p.34-35). I concur with this observation, further suggesting that the technical function of diaphragms at both ends of the transduction chain amounts to a core feature of audio recording technology; that is, an ontological feature of the phonograph. This is an idea I will discuss throughout this thesis.

It should be noted that understanding the term 'documentary' as describing the result of the phonograph's ability to reproduce a past event is a very simplistic 
view. The issues of what is being recorded, the role of the engineer, and the impact of technology in this complex relationship deeply affect a listener's understanding of 'the past event', and therefore understanding the phonograph as a 'document' is far more complex than it might first appear. These intersections between technology, intentionality and 'the work' will be discussed throughout this thesis, beginning here in with a discussion of phonographic genres, particularly classical music recording and popular music recording, to illustrate the types of documents that are commonly produced, and how these relationships operate within them. Before doing so, it is important to acknowledge two important features of all phonograph types, as these features help to clarify aspects of the phonograph that are poietically driven, those that are a matter of composer intentionality, and those which are ontologically distinct, bound to the phonograph itself.

The first feature, as alluded to above, acknowledges a simplified technical understanding of the phonograph, one in which the sound waves of a past reality are reproduced in the present. This technical and temporal feature lies at the heart of all phonograph types. Sound waves modify an apparatus of the recording medium, be it a diaphragm, an engraving stylus, the state of magnet or a digital device in the past, and the same sound waves, though coloured through transduction, storage methods and reproduction technologies, move a diaphragm in the present. It is this technologically mediated relationship between past and present that gives the phonograph its perceived documentary power. I employ the term phonograph-as-document to describe this particular understanding of a phonograph. However, this essential feature is also present in phonographs that have been produced by composers who seek to collapse the distance between the past reality and present audition; that is, to mute this feature by removing a sense of the past altogether. This condition is perhaps best observed in the phonographs of musique concrète (see chapter two). It should be noted that regardless of this intention, an immutable technical and temporal relationship between the production of the phonograph and its replay persists. 
The second feature of the phonograph to indicate before beginning a discussion of phonograph types is related to the first feature. Just as all phonographs connect the past and present through the temporal displacement of sound waves, so to do to they exhibit a discrete relationship; that is, the past event and phonograph itself are ontologically distinct: the phonograph is abstracted from the event. The nature of this abstraction is discussed in chapter three, but it is important to note here that the event and the recording itself (the phonograph) are not the same thing. Here I propose that all phonographic types feature abstraction at this level, and it is this abstraction that brings about the notion of authorship in the phonograph. As such, all phonographs are in some way composed, regardless of the intentions of those making the recordings. I use the term phonograph-as-work to describe this understanding of the phonograph. Composers of the phonograph-as-work strongly promote abstraction, as witnessed in the practice of electroacoustic composition, a genre discussed in chapter two, while composers of the phonograph-as-document, including the recording engineers of classical music for example, hold a more complex position regarding authorship, due largely to the popular conception of the work as nominalist (Goehr, 1992), and the perceived role of the engineer as one whose actions support this notion.

Finally, before embarking on a discussion of the phonograph-as-document to the phonograph-as-work continuum, it must be emphasised that the ontologies described above are universal; that is, in every phonograph there is an element of documentary (phonograph-as-document), just as there is element of authorship (phonograph-as-work), and as such, the continuum presented between the two is useful chiefly from a poietic perspective, as a way to reveal the intentionality of those creating recordings. Though the continuum is primarily a device of intentional investigation, along the way, we encounter subtle shifts in the ontology of the phonograph, born of the technical processes of those creating them. As I will explore, these ontological modifications help to position works along this spectrum. As such, the purpose of presenting a continuum of phonograph types is to highlight common perspectives of the phonograph from a variety of perspectives, while revealing these ontological 
distinctions. It should also be noted that the reproduction phase of phonographic experience is largely absent from the discussion that follows. This is not to say that reproduction is considered of little consequence to phonographic experience (quite the opposite is true), but rather I suggest that despite the obvious importance of playback technology, listeners are able to attend to the formative aspects of the phonograph regardless of which playback technology is employed (an idea discussed in chapter six), and it is to these formative aspects of the phonograph that I will now turn.

\section{Classical music recordings: the phonograph-as-document}

In Capturing Sound (2004), Mark Katz discusses in some detail a commonly held belief in early twentieth-century America. The perception was that 'classical music was a powerful cultural and moral force to which American's sadly lacked exposure, and that technology, perhaps more than any other agent, could foster positive social change' $(2004$, p. 49). The idea that audio recordings have the power to morally enlighten is a widely observed theme outside of classical music, especially in the genre of soundscape composition (see chapter four). Katz goes on to outline the many socio-political reasons for the promotion of classical music recordings over other genres, including its power to grant white Americans cultural parity with their European counterparts, as well as the perception of many white and black Americans that classical music could elevate the social standing of black Americans (p. 52). I would like to consider the ramifications of this early emphasis on classical music recordings on the development of our understanding of the phonograph. To do this, I will now turn to the views of those creating the recordings in order to reveal the complicated relationships between recording technology, performance, authorship, and the work.

Igor Stravinsky's compositional output stretched from the late 1900s until the late 1960s. He began recording his own compositions (as conductor) in the 1920s, not long after the electrification of audio recording, and by the end of his career, the quality of recording technology had reached a very high standard, not 
far from the kind of 'fidelity' modern digital technology offers. The enormous advances of recording technology during his career, along with his own engagement with it as composer and conductor, makes Stravinsky's perspective on classical music recordings an important one. Not only is Stravinsky's perspective important given his close relationship with the evolution of recording technology, but also his perspective is representative of the wider culture of classical music. ${ }^{1}$ It should be noted that Stravinsky was not only interested in recording technology for its ability to capture live performance. In 1930, he wrote of his desire for other composers to create phonograph-specific music, revealing an appreciation of the potential of recording technology beyond the established phonograph-as-document paradigm. ${ }^{2}$ Here Stravinsky has likely identified the second essential feature of the phonograph I have described previously: its ontological distinction from that which is recorded. His call for phonograph-specific music suggests that this distinction is something to be exploited. However, where classical music recordings are concerned, Stravinsky mutes the creative potential of the phonograph, instead promoting the phonograph as a tool for documentary alone, with a dictation-like function similar to that which Edison envisaged in 1877.

In Stravinsky's 1936 autobiography, he recounts the early recordings of his compositions made for Columbia. Of these recordings, which he conducted, Stravinsky writes:

These records, very successful from a technical point of view, have the
importance of documents which can serve as guides to all the executants of
my music. Unfortunately, very few conductors avail themselves of them.
Some do not even enquire whether such records exist. Doubtless their
dignity prevents others from consulting them, especially since if once they
knew the record they could not with a clear conscious conduct as they liked. (Stravinsky 1936/2012, p. 107)

\footnotetext{
1 Arved Ashby's Absolute Music, Mechanical Reproduction (2010) and Mark Katz' Capturing Sounds (2004),

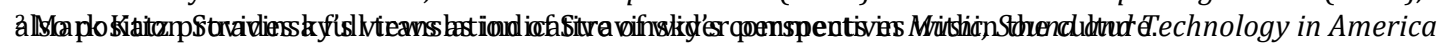
(2012, p. 113)
} 
This statement reveals a great deal. To start with, Stravinsky clearly thinks of his recordings as documents; that is to say, he appreciates the same essential feature of the phonograph that I have previously identified as ontological. This is certainly not an uncommon view, although there are some unique aspects to his perspective. He states the recording is a 'guide' to the 'executants' of 'my music'. To begin, the recording is termed a 'guide', which amounts to, as Arved Ashby writes, 'a function of his published compositional text' (2010, p. 201). In this way, his recording is like a score, or rather, it holds the same status as the score in relation to his compositions. His reading of recording as score-like is confirmed by Stravinsky's desire for other conductors to consult his recordings in order to gain an understanding of how they should be performed. This leads to an important distinction: Stravinsky sees his role in these recordings as that of composer, not conductor. Only his recordings can be viewed as true documents of his works. Other conductors ("executants") are making inadequate renditions of his compositions because they do not know Stravinsky's purportedly definitive recordings. It follows then that Stravinsky understands recordings of compositions as different kinds of documents depending on the status of the conductor: his recordings are score-like documents of his compositions, while other conductors create documents of (inferior) performances of his compositions.

Stravinsky also suggests that many conductors assume some authorship of the work: "'have you heard my fifth, my Eighth?" - that is a phrase that has become quite usual in the mouths of these gentlemen' (1936/2012, p. 108). Here we see that Stravinsky, to some degree, holds what Lydia Goehr (1992) describes as a 'nominalist' view of the work, a term I will discuss shortly. As the comment above attests, Stravinsky believes that such conductors do not have access to the composition on the same level as the composer. Further to this, it appears that Stravinsky understands his own recordings act to support the integrity of his compositions while others degrade it, a position I will discuss shortly. This is an important evaluation, as these nominalist views of the work persist in a great deal of recordings and productions, both classical and otherwise. It is an understanding that causes some tension in practices (not least of all 
environmental sound composition) in which the phonograph is afforded a higher creative function than simply that of phonograph-as-document. For the moment, however, I will consider established approaches to the work-concept in specific relation to classical music.

\section{The nominalist work}

As Goehr writes in The Imaginary Museum of Musical Works (1992), a common reading of the work is that it is abstract in form. Though many composers may consider musical works as abstract in a general sense, Goehr's account of the abstract work provides a level of specificity that is useful to explore, as these readings help to further define the possible relationships between the work and the phonograph where composer intentionality is concerned. Of these readings Goehr details three conceptions: the Platonist view, The Aristotelian view, and the Ideal view, each of which she considers as existing under the umbrella of the nominalist view. Central to this conception of the work is that it is both universal and singular. Of the Platonist view, she writes:

In one of its articulations, musical works are argued, contrary to common sense, to be universals - perhaps even natural kinds - constituted by structures of sounds. They lack spatio-temporal properties and exist everlastingly. They exist long before any compositional activity has taken place and long after they perhaps have been forgotten. They exist even if no performances or score-copies have been produced. To compose a work is less to create a kind, than it is to discover one. (1992, p. 14)

In Goehr's discussion, Jerrold Levinson's modification of this view holds the work dependent upon 'human intentional act for them to come into existence, and performances and scores for them to stay in existence' (1992, p. 15). The work itself however, remains distinct from such dependents, aligning Levinson's definition with the Platonist view in positioning the work as abstract. The Aristotelian view posits that the existence of a work is dependent upon its performance and indistinct from such a performance. Like the Platonist view, the work exists in the abstract, but only during and as a result of performance. In this 
sense, an Aristotelian view holds that a distinct work exists as 'unactualized or unsubstantiated potential' (p. 16). The Idealist view, perhaps the most widely held yet unexamined reading of the work, holds that:

\footnotetext{
Works are...ideas formed in the mind of composers. These ideas, once formed, find objectified expression through score copies and are, thereby, made publicly accessible. (1992, p. 18)
}

On this view, works only exist in the mind of the composer; therefore scorecopies are not creations, but limited actualisations. In this way, a score only grants limited access to the work. I argue that Stravinsky holds a nominalist view of the work, possibly Idealist in nature, as he believes his scores are inadequate guides for other executants. In Stravinsky's view, a composer-conducted performance allows greater access to the work, and presumably, a composersanctioned recording of this performance does also. Regardless of Stravinsky's exact position in the nominalist spectrum, he positions the work in relation to himself: he alone has privileged access to it. The inadequacies presented by his score, by the performance, and by the recording itself, can be attended to and refined by Stravinsky in order to achieve the closest possible rendering of his work in relation to the work. Stravinsky's disparaging comments regarding the tendency of conductors to assume a level of authorship of works they conduct, certainly attests to this perspective. Where the composer is not the executant of the performance, it follows that such a performance acts as both an actualisation and a distortion of the work, as a conductor only has access to the score, itself a limited actualisation of the work. A recording of such a performance might then be understood as a further distortion and actualisation of the work, though, in the case of Stravinsky, it is unclear whether he considered a recording to have any relationship to the work beyond that of a guide. Regardless of the specificity of this relationship, for Stravinsky, we might conclude that a non-composer conducted and sanctioned recording of the work is three-times removed from the work itself, as both the score and the performance stand between the two. This would hold true for all three nominalist positions. 


\section{The Analytical work}

Goehr notes a fourth conception of the work dubbed the Analytical approach, which holds that the work has no form of abstract existence. While this view is not as widely held by composers of western art music of the nominalist approach, it is nonetheless an important view, and as I will discuss later in this chapter it has relevance to other recording genres:

\footnotetext{
Here one moves away from the vertical relation between a work and its performances, a relation obtaining between an abstractum and its concreta. One considers, instead, the horizontal relations obtaining between performances and score-copies. (1992, p. 17)
}

The Analytical view can be seen to take emphasis away from the composer/work relationship, shifting focus instead to the work and its actualisations. We might consider many works of the latter half of the $20^{\text {th }}$ century to more closely align with the Analytical perspective, in that chance procedure and improvisation in particular began to feature more strongly in compositions. ${ }^{3}$ Though many of these works may not be widely associated with nominalism (Umberto Eco's The Open Work (1989) provides a popular alternative reading of such works), they nevertheless may be classified as Analytical in nature. Moving further still from the composer/work relationship, we might argue a work that exists entirely between its concrete form and the listener, with limited or no recourse to the composer. Roland Barthes makes such an argument in his essay 'Death of the Author' (1967) in the context of reader-response theory, discussion of which is central to chapter six. For now, however, I will posit that both abstract and concrete readings of the work are important to the discussion of the phonograph in general terms, but it is nominalism in particular that can be seen to influence the production of classical music recordings, insofar as the phonograph is considered distinct from the work itself, often functioning as an actualisation of the work, similar to that of a score. Nominalism can also be seen to inform the

\footnotetext{
3 The chance procedures employed by John Cage in Music of Changes (1951), or the performer-focused and improvisatory nature of many Minimalist works, including Terry Riley's In C (1964) are exemplary of alternative readings to nominalism that can be understood as Analytical in nature.
} 
ideologies and practices of many early composers of electroacoustic composition (the subject of chapter two), and as such, nominalism is significant to the discussion of the both the phonograph-as-document, and the phonograph-aswork.

Having established that a popular understanding of the work in classical music is nominalist in conception, it follows then that, like a performance and a score, a good recording is required to have a degree of fidelity to the work. The desire for 'technological transparency', a notion in which the presence of the phonograph itself is muted in favour of the event it captures, is strong within the classical music recording community for this reason. As such, classical music recordings typically present the phonograph-as-document type. Barry Truax conceives of technological transparency as the "black box" model of electroacoustics:

The signal enters and exits from the box via the transduction processes which converts the energy from acoustic to electrical forms and back again... The aim of the system is to achieve "fidelity" between the input and output signals, that is, to make them indistinguishable, at least ideally. (2001, p. 8)

This idealised view is deeply problematic, as Truax rightly identifies:

Perfect fidelity, of course, is technically impossible to achieve because every stage of the signal transfer process inevitably adds noise or distortion to the signal, however slight. (p. 8-9)

Truax identifies a very important notion: recording technology imparts its 'sonic signatures' to the phonograph, and such a process is inherent in all recordings. ${ }^{4}$ It should be emphasised that the 'sonic signatures' of technology are apparent in each of the three aspects the phonograph: its production, its storage medium and the replay technology employed. Though Truax conceives of such signatures as 'noise or distortion', which, in the context of "fidelity" may be appropriate, the

\footnotetext{
4 The term 'sonic signatures' and research into its meaning is becoming more widespread. The Sonic Signatures Symposium at Aalborg University in April 2014 is testament to this growth. See http://www.sss.musik.aau.dk/ (accessed 16 September 2015)
} 
sonic signatures of recording technology are far more complex than such a conception suggests, and, as I will explore in chapter six, these signatures constitute a rich vein of listener engagement well beyond the negative connotations the terms 'noise' and 'distortion' encapsulate. For now, it should be emphasised that perfect fidelity, though impossible, is the goal of many classical recording engineers in pursuit of the closest representation of the work.

In order to achieve the closest fidelity to a work, composers, ensembles and engineers have often opted for single take, single room recordings of classical ensembles, usually referred to as live recordings. Historically, this particular focus grew from the technical limitations of early audio recording. Prior to multitrack magnetic tape, orchestral works had to be recorded without edits or overdubs, typically using only a few microphones at most. Though many composers and recording engineers have embraced technology since the 1950s, ${ }^{5}$ many strictly acoustic works continue to be recorded in single takes in a single room, using a main stereo pair of microphones to capture an appropriate balance of ensemble and room acoustic. Today, such a approach might be considered "purist", indeed many ensembles are no longer recorded in this way, especially in the area of film music, ${ }^{6}$ but where classical composition is concerned, this focus on the performance, the performance space, and the desire to capture it in one span of time, remains common. Manufacturers of recording technology designed for classical music often use the terms such as 'clean' and 'transparent' to describe the performance of their equipment. ${ }^{7}$ It should be noted that 'transparency' encapsulates two ideas where classical music recordings are concerned. As mentioned, the aim of phonographic transparency in classical music recording is to grant the best possible access to the nominalist work. It

\footnotetext{
5 The use of amplified instruments, overdubs and added artificial reverb are some the techniques used; George Crumb's 'Black Angels' (1973) and the Kronos Quartet's recording of it (1990) are exemplary of such techniques

${ }^{6}$ John Neil, the Head of Sound for Peter Jackson's film The Hobbit: An Unexpected Journey (2012) recounted using more than 40 microphones for the capture of the New Zealand Symphony Orchestra during a lecture I attended in 2014.

7 By way of example, DPA Microphones, a company highly regarded by classical engineers for their 4006A model microphone, boasts 'clean, natural and precise reproduction...[with] high sensitivity, low noise-floor and extremely linear frequency response...ideal for A-B stereo pairs for symphonic concert hall recordings'. This language is not unusual for professional microphone companies, and regardless of the microphone's actual performance, it shows that these are values held by their customers: http://www.dpamicrophones.com/en/products.aspx?c=Item\&category=123\&item=24512 site accessed 12 December, 2014
} 
does this by muting the sonic signatures ('noise', distortion') of the technology employed, while promoting a sense of realism (spatial, timbral and dynamic fidelity to the source), the second kind of transparency. The focus on realism in recording, like the muting of the technology's sonic signatures, is designed to bring the listener as close to the work as possible. Many classical music recording techniques are designed to make a listener feel as if they are in the room with the ensemble. Techniques including Alan Blumlein's stereophonic microphone arrays developed in the 1930s, and binaural 'dummy head' microphone systems, were developed with this goal in mind. More recently, ambisonic recording techniques have gained popularity in both classical music and film music recording, perhaps as a result of the proliferation of surround sound in home theatre systems. In this way, we can still observe the desire for transparent documents within the genre. There are some instances in which the notion of transparency and purity in classical music recordings are challenged. This was most pronounced with the advent of magnetic tape, a format which introduced the technique of splicing; an editing technique in which the phonograph moves from one recorded performance to another, usually maintaining the illusion that the two (or more) performances are in fact one continuous take. As Brian Eno notes:

The move to tape was very important, because as soon as something's on tape, it becomes a substance which is malleable and mutable and cuttable and reversible in ways that disks aren't. (Eno 2004, p. 128)

Where the nominalist work is concerned, we might conclude that editing takes a recording yet another, fourth, step away from the work itself, which is where the opposition to editing finds its roots. The question must be asked: does editing a performance distort the performance, thereby further distancing a recording from the work? Or does editing, in instances where the edits are undetected by the listener, actually bring the recording closer to the work? For Glenn Gould, the inconspicuous edit, rather than introducing distortion, allows greater fidelity between the performance and the score. Furthermore, where the performer is 
also the editor, their privileged relationship with the score can be further strengthened:

\footnotetext{
When the performer makes use of this post-performance editorial decision, his role is no longer compartmentalized. In a quest for perfection, he sets aside the hazards and compromises of his trade. As an interpreter, as a gobetween serving both audience and composer, the performer has always been, after all, someone with a specialist's knowledge about the realization or actualization of notated sound symbols. It is, then, perfectly consistent with such experience that he should assume something of an editorial role. (1966/2004, p. 118)
}

Regardless of whether Gould adhered to an abstract (nominalist) or concrete (analytical) conception of the work (for post-performance splicing can be seen to strengthen both conceptions), the inclusion of the splice affects our understanding of phonograph-as-document from an ontological perspective, as the resultant phonograph can no longer be understood as a recording of a singular event, though a listener may be unaware of this modification. In instances where one recording is spliced with another, the question of what is being documented immediately arises. When splicing is present, the phonograph-as-document changes at a technical level; no longer does the loudspeaker reproduce the sound waves of a previous time, but it reproduces sound waves of previous times: there are now multiple temporal relationships between the past and present. In this way a single take recording might be considered a reproduction of a sonic event, while a spliced recording represents a synthesis of sonic events. In my view, the splice, that is, a temporal difference between the framed event and the recording, represents a subtle, often undetected, yet substantial modification in a phonograph's ontology: though the essential documentary feature remains, insofar as all materials in a recording have been captured in the past, 'the past' is no longer a singularity, though it may be perceived as such by a listener unaware that a splice has occurred.

The second thing a splice produces is the unequivocal emergence of a kind of new performer. Glenn Gould, in splicing together his performances of Bach's 
Fugue in A Minor (as he discusses in his 1966 article 'The Prospects of Recording"), believes post-performance splicing to be a function of his total recorded performance. In other words, as an executant of Bach's work, Gould uses recording technology to render a performance (albeit a 'simulation' of one as Baudrillard [1994] would have it), as close to Bach's score as possible. I suggest that Gould's use of the splice is certainly an aspect of his performance, but even more than this, the splice begins to align his role with that of a composer, as his authorship of the phonograph becomes more acute. To call an editor of classical music a composer is unusual, as this term is often reserved for the person who 'writes' the instrumental work. The classical music editor's role on most occasions is considered one of support: to make a recording sound transparent, in service to the nominalist work. This is also true of the classical music engineer. Where phonograph-specific works are presented, such as those of the electroacoustic music tradition (see chapter two), the editor can hardly be viewed as anything other than a composer. As such, though the editors of classical recordings and the composers of electroacoustic music are widely considered to hold different relationships to their respective works, they both exhibit a degree of authorship where the phonograph itself is concerned. A common difference between the two is that the electroacoustic composer may wish their authorship to be perceived by a listener, while a classical music engineer may not. From a listener's perspective, the presence of the engineer typically becomes more pronounced the more explicit their manipulation of the recording becomes. The more explicit their manipulation becomes, the further we move along the continuum towards the phonograph-as-work.

By way of summary, I suggest that in classical music, the wide-spread and enduring perceptions of the work and the role of recording have a specific relationship, which amounts to a class or genre of recording which, as I will demonstrate, permeates other recording practices to varying levels. To begin with, classical music embraces the concept of the phonograph-as-document as the dominant reading of its function. This is related to the enduring nominalist understanding of the work as discussed by Goehr (1992). In essence, the classical recording offers access to the work by providing access to the 
performance, and this is considered its primary value. The performance is either understood as an actualisation of the work (to varying degrees according to Stravinsky), or the work itself (where an abstract reading of the work is rejected in favour of an analytical approach). Regardless of which reading is adopted, transparent access to the performance is desirable, to the extent where even subtle, undetectable editing may be considered a degradation of the phonograph's perceived primary function, understood as a type of aural document of a singular event. This is a matter of authenticity, and classical "purists" might argue that an edited performance is a kind of counterfeit document. As mentioned above, regardless of whether manipulation is detected or not, it is my belief that splicing amounts to a significant modification in the documentary aspect of the phonograph's ontology. If we establish that temporal multiplicity in a recording can exist as a result of the splice, regardless of their perception at audition, we can then assert that such a condition represents a subtle ontological distinction to phonographs which represent a singular temporal frame.

As mentioned earlier in this chapter, the continuum from phonograph-asdocument to phonograph-as-work is largely relevant from a poietic perspective, insofar as 'document' and 'work' are essential features of all phonograph types. Where the splice in classical music recordings is concerned, however, a production method designed to strengthen the documentary aspect of the phonograph at an experiential (esthetic) level, by providing the highest possible fidelity to the score through the best possible 'performance', actually changes the phonograph at an ontological level. Put another way, although classical music recording engineers attempt to present the phonograph as a realistic and transparent document of a performance, in part through the concealment of abstraction through its 'hi-fidelity' recording techniques, it uses further abstraction (the splice) to achieve its goals. As such, though the recording may sound like a live recording at an experiential level, it is in fact further abstracted at an ontological level than a true live recording. For this reason, the splice represents a step away from the single take, single room recording, into a 'pseudo-document'. The term is borrowed from Michael Dellaira (1995) and 
denotes a phonograph that, though composed of temporally distinct performances, is made to sound live. The splice announces the transition from the live recording (phonograph-as-document) to the pseudo-document, itself the first step along the continuum to the phonograph-as-work. I will now turn to the genre of popular music recording, of which there are many subgenres, as the territory within which to explore these various document types.

\section{Popular Music recordings}

Before further exploring the pseudo-document within the realm of popular music, it is important to consider what popular music might be, and the role of technology within it. To begin, it must be emphasised that popular music (in general terms) has an interesting and complex relationship with recording technology. At times, popular music recording, as a genre, shares the same kinds of relationships between technology and the work as those presented by classical music recording. This reading is one of many. More often a complex mix of composition, performance and production can be observed in popular music, with the respective roles of composer, performer and producer intermingling, often inseparably from one another. As such, the space between the phonographas-document and the phonograph-as-work is a rich and varied space, within which popular music recording genres often freely traverse.

Of course, defining 'popular music' is a difficult task, which I cannot undertake here. For the purposes of this thesis I will broadly define popular music recordings as those that use the phonograph as a way to engage with the audience, and where concerns regarding the nature of the work and how it should be approached are secondary concerns to this dynamic, reflexive engagement. In contrast, classical music recording is fundamentally tied to the nominalist work concept, and thus such audience reflexivity through recording is a secondary concern of the genre, if it is a concern at all. While in some instances popular music engages with the 'live recording' format of classical music, it should be noted that this approach is generally decreasing, due in part to the to the growth of smaller home studios equipped with inexpensive computers and 
software, and the diminishing number of large, commercial studios as a result. ${ }^{8}$ The rise of the small home studio is economically and technologically driven; it is much more affordable to produce music using relatively inexpensive and compact technology at home than it is to use large studios, studios that are designed to record multiple instrumentalists and large ensembles at once, often with expensive and sizable hardware. An increasing number of popular music producers are turning to software-based instruments for their pieces, including ensembles too large to record in smaller spaces such as orchestras and large drum kits. In classical music, though software is often used as a tool in the creation of scores, it is unusual for software instruments to be recorded in place of a real performer; classical music recordings continue to strive to offer transparent access to a live performance and a composer's work, maintaining the long-established hierarchy of the work, the composer and the audience. As such, classical music recordings have almost exclusively utilised the live-recording format, especially for larger ensembles, where producers of popular music recordings have adapted to the changing technological and commercial environment.

In the case of some popular music, such as the manufactured girl and boy bands of the UK, USA, and Korea, the establishment of a reflexive relationship with the audience is the only principle of its production. This is perhaps most clearly seen in music designed for mass sale, where tempo, instrumentation, duration and production techniques are largely informed by what is currently selling, or what is thought likely to sell. Large record companies often hire songwriters and production teams to produce recordings for their singers on the basis that these individuals have "their finger on the pulse". Television variety shows including XFactor and American Idol take this model to an extreme, insofar as the audiences vote for their favourite artists, with the artists often recording the songs that

\footnotetext{
${ }^{8}$ Andrew Leyshon's paper "The Software Slump: digital music, the democratisation of technology and the decline of the recording studio sector within the musical economy" (2009), explores the decline of large studios in these terms.
} 
made them winners. ${ }^{9}$ Other forms of popular music, even those perceived as vehemently opposed to commercially-focused practices, nevertheless respond to their audiences in a similar manner. For example, many punk music recordings, much like classical music recordings, are understood as a way to grant an audience access to a performance, though in the case of punk, a 'DIY' 'lo-fidelity' recording is often considered preferable to the 'hi-fidelity' standards of many classical music recordings. In many cases, much like classical music recordings, punk recordings require appropriate access to performance; that is, a commitment to accurately present the qualities of a punk sound, yet unlike classical music, punk is adverse to the assumed transparency of hi-fidelity; distortion, imbalance, extreme dynamics and noise are often hallmarks of authenticity for a critical punk audience. Recordings engineered by Steve Albini, a divisive figure in Chicago's punk and hardcore scene since the early 1980s, are exemplary of this duality:

On the Jesus Lizard albums Albini recorded, singer David Yow sounds like a kidnap victim trying to howl through the duct tape over his mouth; the effect is horrific. The recordings were both very basic and very exacting: Albini used few special effects; got an aggressive, often violent guitar sound; and made sure the rhythm section slammed as one. (Azerrad, 2001, p. 344)

The 'basic' and 'exacting' sound of Albini's records reveals a great deal about his recording practices. In many respects, his recordings are not aesthetically dissimilar to those of classical recordings: he records bands live in the same room while using a minimal range of microphones and limited effects. However, during the mixing of these recordings, Albini often buries the vocals beneath the drums and guitars, which are often heavily distorted and prone to wide variation in dynamics. In effect, while Albini strives to capture the sonic experience of a live ensemble in a documentary way, he also exaggerates the live experience; affronting the audience with loud instruments, while requiring that they actively search for the human voice through the volatile mix. Albini insisted that one of

\footnotetext{
${ }^{9} \mathrm{X}$-Factor UK winner James Arthur's 2012 cover of Birgisson and Wroldsen's song 'Impossible' (2010), was performed during the show, then released shortly after its conclusion, selling 187,000 downloads in the first 24 hours. See: http://www.independent.co.uk/arts-entertainment/tv/news/james-arthurs-impossiblebecomes-fastestselling-x-factor-single-8406195.html accessed 6/11/2014)
} 
his recordings should "grab you by the face and drag you around the room...it should jump out of the speakers at you" (2001, p. 344). Albini desires that the confronting and visceral sound of a live punk band should be perceived in his recordings, which he attempts to simulate through his exaggerated mix techniques.

Albini's recordings are perhaps more 'hi-fidelity' than other recording engineers of the punk genre. In the 1980s, it was not uncommon for albums to be recorded on inexpensive 4-track tape recorders or tabletop 8-track reel-to-reel recorders, cheaply and quickly. Jack Endino, the preferred engineer for Seattle-based record label Sub-Pop, famously recorded Nirvana's first album Bleach (1989) on an 8track machine in 3-days for little over $\$ 600$ dollars. ${ }^{10}$ Though Nirvana achieved mainstream commercial success after the release of their second album Nevermind (1991) arguably due in part to a shift in production techniques, Bleach has the sound of an album recorded within the culture of 1980s American underground punk and hardcore scenes. This fast-paced, lo-fidelity approach is as much about aesthetics as it is about politics and in the case of punk genres, the two are deeply related. ${ }^{11}$ Major record labels often have large recording budgets for their artists, which can result in a very hi-fidelity sound, as engineers use the best equipment and producers can afford to spend time getting the best performances from the musicians. Punk positions itself in opposition to mainstream music, and a low budget, raw sound is a manifestation of this ideology. It is also a direct result of the financial constraints of non-mainstream music production, so the low budget sound is a reflection of a low budget reality, and a low budget reality is in turn a reflection of a band's underground status. As a result, mainstream bands (with access to large budgets) may opt for a lofidelity recording in order to access the sonic signatures associated with underground music. ${ }^{12}$

\footnotetext{
10 The recording of Bleach has been recounted in numerous publications, including Jack Endino's page at Allmusic.com.

11 This relationship can also be viewed in the genres of 'noise' music and the wider culture of cassette tape production. See James, Robin (Ed.) Cassette Mythos (1992)

12 This was the case with Nirvana's third album In Utero, recorded with Steve Albini in 1993, in response to the mainstream success (and underground criticism) of their second album Nevermind. Michael Azerrad, author of Come as You Are: The Story of Nirvana (1993) discusses the recording of In Utero and the controversy surrounding its 'remixing' in detail.
} 
Given that punk recordings champion the live performance over extensive studio multi-tracking and engineering, we might - counter intuitively - consider this genre of recording in much the same light as classical music recordings: the focus is still on 'the performance', though classical music strives for perfection where punk music champions imperfection. The two genres share the notion that a recording should primarily focus listeners to the performance. Though the lofidelity ethos of punk music and the hi-fidelity ethos of classical music seem to suggest that the role of technology in the relationship between performance and audience is understood differently between the genres, they are in fact strikingly similar. As discussed, classical music, through careful engineering, attempts to render recording technology 'transparent'; ideally, technology is conceived as silent, without 'distortion' or 'noise', leaving no sonic signatures. Punk, however, hears careful engineering as anything but transparent; the sonic signatures of hifidelity recordings are clearly heard. In other words, the sound of a clearly defined stereo soundstage, with full-range capture of the instruments, in a spatially and dynamically balanced mix, reveals just as many sonic signatures to a punk audience as classical listeners encounter in a typical punk recording. Punk transparency requires, at the very least, a level of indifference to fidelity if not a requirement for lo-fidelity. As such, punk audiences are able to attend to the performance of a recorded punk band (thus rendering the technology 'transparent'), so long as the recording is not hi-fi. Classical audiences do the same: they hear the technology as transparent, so long as it is not lo-fi. Regardless of these finer points, it is important to note that though certain genres may appear to hold different perspectives on recording technology and what it offers, there is in fact a great deal of common ground between them. Where the pseudo-document is concerned, the commonality between classical music and popular music is particularly strong.

\section{The pseudo-document}

Michael Dellaira, in his essay "Some Recorded Thoughts on Recorded Objects" (1995), defines a pseudo-document as a recording that 'imitates' a document (p. 
14). As noted earlier, Baudrillard might understand the splice as introducing the notion of simulation in the production process, making the resulting phonograph a simulacrum:

\begin{abstract}
ion today is no longer that of the map, the double, the mirror or the concept. Simulation is no longer that of a territory, a referential being or a substance. It is the generation by models of a real without origin or reality: a hyper real. The territory no longer precedes the map, nor survives it. Henceforth, it is the map that precedes the territory...it is the map that engenders the territory. (1995, p. 1)
\end{abstract}

Understood in this way, the pseudo-document truly represents a marked step away from the live recording. At a technical level, the pseudo-document represents a synthesis of two (or more) past events. At a semiological level, the pseudo-document represents a hyper reality: the simulation of an event that never occurred. This hyper reality is evident in many of the recordings of Stevie Wonder, specifically those in which he plays all the instruments. ${ }^{13}$ Dellaira writes:

We are still able to suspend disbelief and find a context for his performance - that is, we can pretend it happened in the only way music ever could happen before the advent of recording machines - in real time and in person. (1995, p. 14)

Dellaira's example of this multi-layered, single-performer recording is a specific type of pseudo-document, one that became increasingly common through the 1970s and beyond. It is undoubtedly a prevalent kind of phonograph, especially in this age of readily available cheap software as recounted earlier. I would now like to consider earlier recordings that represent a different kind of pseudodocument, a type that is arguably more concerned with feigning live performance than representing the multiple talents of an individual artist, as the example of Stevie Wonder would suggest.

13 While Dellaira never mentions a specific track, Stevie Wonder is credited as the sole instrumentalist on a number of recordings beginning in the early 1970s. 
According to Jim Cogan and William Clark (2003), Capitol Studios in Hollywood had an Ampex 200 tape recorder as early as 1948 (2003, p. 20). The tape machine allowed orchestral recordings to be made, and a vocal (often performed by the likes of Dean Martin, Jerry Lewis, and Nat Cole), to be recorded at a later recording session. As far as the public were aware, there was nothing to suggest that the songs emerging from Capitol were not recorded live. This type of pseudo-document was typical of major record companies in the 1950s, and while the new technology offered a whole new range of possibilities (possibilities explored within the realms of art music and radio), it could be argued that the 'live ensemble' pseudo-document upheld the assumed, well-established relationship between ensemble, phonograph and audience, as the vast majority of popular music recordings made before the 1950s were of live performances. In essence, the simulation inherent in multi-tracking during the 1950s attempted to feign a phonograph-type (the phonograph-as-document) that had a history of commercial success; as far as record companies were concerned, 'live performances' sold records, and multi-track technology simplified and streamlined recording sessions, while creating the illusion of an ensemble and singer performing as one. By the 1960s, 4-track recorders were common in America and increasingly in the UK, and by the mid-1970s, 16 and 24-track recorders were standard in most major studios around the world. The growth in track-count during this time also mirrors the growth in studio experimentation, and while new types of phonographic documents would emerge as a result, the desire to present a phonograph-as-document through simulation was still strong among many producers.

Phil Spector, for example, who worked largely with 4-track machines from the late 1950s, developed his well-documented 'wall of sound' recording technique as a way to enhance recordings of studio performances. ${ }^{14}$ Though the unique sound of The Ronette's “Be My Baby" (1963) was a direct result of studio experimentation, as far as listeners were concerned, despite the radical manipulation, they were still listening to a phonograph-as-document of a 'live'

14 Phil Spector's engineer Larry Levine gives a detailed account in an interview with Richard Buskin (2007). 
performance. This kind of simulation was well established by 1963, but his pseudo-documents are interesting from another perspective. Spector's particular brand of hyperrealism involves overt technological experimentation, and as Spector's studio engineer Larry Levine recalls, "the things Phil was doing were crazy and exhausting...but that's not the sign of a nut. That's genius" (Levine in Ribowsky, 1989, p. 149). Spector's experimentation within the studio imparted a sonic signature that others were quick to explore and extend, not least of all the Beatles, as I will explore shortly. The important thing to note is that throughout the 1960s, beginning with producers like Spector, the notion of the sonic signature began to feature more heavily in popular music recordings. Though every recording presents such signatures, including the examples of classical and punk recordings recounted earlier, the overt exploration and promotion of a recording's sonic signatures within the pseudo-document augments the notion of it. It does not change the pseudo-document at a fundamental level - Spector's recordings were still simulations of live recordings - but the promotion of the studio and its technology in his work can be seen to suggest an intermediate presence in the work; that of the studio and the producer himself. In some respects, Spector's pseudo-documents are not dissimilar to Gould's classical pseudo-documents, though where Gould sought to mute his presence through the subtle, undetectable splice, Spector's presence is keenly felt in his use of extensive overdubbing and spatial effects. As such, Spector began to reveal his role in the process of abstraction, thus assuming a heightened level of authorship in the recordings he created.

This type of pseudo-document foreshadowed the arrival of a new type of document - the meta-document - a type of phonograph that is dependent on studio manipulation, though it does not simulate 'live recordings' as the pseudodocument does. Before moving on to this next kind of document, I would like to point out that the Spectorian pseudo-document continues to be a popular choice for many musicians, engineers and producers of popular music, especially where the presumed, primary focus of the audience is on the performance, not the recording itself, though in many cases, the recording becomes just as revered for its production as the performances contained therein. Such is the case with 
Spector's “Be My Baby”. Other examples include My Bloody Valentine’s Loveless (1991), Smashing Pumpkin's Siamese Dream (1993) Radiohead's OK Computer (1997) and Beck's Sea Change (2002). Each of these recordings present a series of pseudo-documents in which overdubbing has featured strongly, creating hyper real recordings of groups that sound markedly different in a live context.

\section{The meta-document}

During the 1960s a new kind of document emerged. I use the term 'metadocument' to describe this, understood as an extension of Dellaira's 'pseudodocument'. Like the pseudo-document, the meta-document contains live performances, but the conspicuous use of studio processing creates a recording that cannot be interpreted as solely 'live'; our ability to 'suspend disbelief', as Dellaira wrote of Stevie Wonder's recordings, is compromised by features of the recording itself. The meta-document is thus situated between an appreciation of the performance (phonograph-as-document) and the recording itself (phonograph-as-work). Although it contains elements of both, it cannot simply be understood as one or the other. While there are many examples of the metadocument from the 1960s onwards, perhaps the best-known examples are found in the later recordings of The Beatles.

The studio techniques utilised by The Beatles, George Martin and the engineers at Abbey Road in the 1960s are very well documented. ${ }^{15}$ The Beatles' recording career began with a single take, single room recording of "Love Me Do" in 1962 and moved through to thoroughly studio-constructed compositions. As a result, their oeuvre represents a variety of different kinds of phonographic documents. In his book Capturing Sounds (2004), Mark Katz discusses the production of The Beatles' “Strawberry Fields Forever” (1967). Katz' analysis centers around the splice, in which two performances were edited together, with one slowed down a little, giving John Lennon's voice a 'distinctively dreamlike quality' (2004, p. 41). He also notes of the unique spatialisation of the instruments on 'the stereo stage',

15 Recording the Beatles (2006) by Kevin Ryan and Brian Kehew is arguably the best account of the Beatles recording practices during the 1960 s. 
identifying that a slide guitar crosses the sonic stage from left to right around 15 seconds into the recording. Katz concludes that the space created in "Strawberry Fields Forever" has 'no possible physical counter part' (p. 42). As such listeners who hear the version in stereo will realise, if they haven't already, that they are not simply listening to the recording of an event, but that technology is augmenting the performance. While this stereo sound stage, understood here as the width of the stereo field, is completely lost in mono, ${ }^{16}$ other studio manipulations in "Strawberry Fields Forever" are much more apparent, in both mono and stereo. The coda of the song, from about 3'35", evidences another feature of the meta-document. The mellotron in this section is subject to tape reversing, an effect that instates a clear division between the original performance and the recording. Unlike Spector, who used the studio to enhance performances, the Beatles used the studio, in this instance, to create a performance that could not be achieved without the technology used. For many listeners, the reversing effect announces a process that is not achieved by way of the mellotron's 'standard performance', a standard established in the first 3 minutes of the song itself. It should also be noted that reversing is also used in the main body of the song (on the cymbals from 1'21" - 1'41"), which adds to our appreciation of the mixture of instrumental performance and studio manipulation.

Another extended, experimental coda can be found on another Magical Mystery Tour song, "I am the Walrus". This section includes the sound of a radio dialing through frequencies heard over dense string orchestration. Much like "Strawberry Fields Forever", this coda acts as a kind of experimental montage after the main body of the song, in which the studio manipulations are showcased over and above of the performances. On their next album, Revolver (1968), The Beatles include a track that consists primarily of these techniques, "Revolution 9", which contains a number of different performances (some by The Beatles, some taken from other recordings) and assembled in a manner not dissimilar to the works of musique concrète (discussed in chapter two). This

\footnotetext{
16 Magical Mystery Tour was released in both mono and stereo in 1967, which illustrates the tenacity of
} mono in the late $1960 \mathrm{~s}$ 
particular track represents a movement away from the meta-document status characteristic of many Beatles recordings of the time and closer to that of the phonograph-as-work paradigm.

The type of meta-document found in late era Beatles recordings, as exemplified by "Strawberry Fields Forever", is similar to the pseudo-document as typified by Phil Spector's "Be My Baby”, insofar as both recordings exploit the sonic signatures of studio equipment and processes in order to create hyper-real 'performances' on record. Where the two differ, is that the studio experiments found in Beatles recordings no longer attempt to simulate a live performance; the studio experimentations - especially reversing effects - are distinctly studiobased manipulations. As such, this type of meta-document can be seen to significantly extend the kind of pseudo-document prevalent in the early 1960s. With an awareness of the studio manipulations that occurred after the initial performance, the audience may be led to perceive the duality of the metadocument: The Beatles are present in the instrumental performances, and they are also present in the manipulation of those performances, in the studio, at a later time. As a result of this, a listener may also become aware of a new temporal dimension, understood as a kind of dislocation between the performance and the production, though in the case of The Beatles, awareness of these temporal relationships may be hard to perceive. This is due in part to their role in both the performance and production of their recordings. In a similar manner to Gould, The Beatles are considered executants of their own recordings and thus the inherent dislocation between performance and recording may be collapsed in the appreciation of their total recorded performance. This is an idea I will discuss in relation to dub music production in the next section. Indeed the temporal aspects of the phonograph are a core topic in this thesis and will become central to later chapters.

In many respects, the cut and paste montage of "Revolution 9" can be seen as one of many precursors to another kind of meta-document; a kind that came to prominence in the 1980s and 1990s in the form of hip hop. Before exploring this genre, it is important to consider the kinds of phonographs being created in 
Jamaica during the 1970s, as the culture surrounding Jamaican music dissemination, along with the kinds of techniques employed in the production of 'dub' music, would directly influence early hip hop music emanating from the Bronx in the late 1970s. In addition to this, dub music presents a very unique and influential form of the meta-document, which continues to inform the production methods of many different genres.

\section{The phonographs of Jamaican popular music}

The reflexive relationship between the recording artists and their audience, mediated by the recording (as discussed earlier in this chapter), is so prominent in the development of Jamaican popular music that charting the role of technology independently from social, cultural and political factors is a difficult undertaking. ${ }^{17}$ As my interest in Jamaican popular music in the context of this thesis is primarily concerned with recording and technology, I will provide only a brief overview of the relationships between the audience and artists before discussing the development of the phonograph within this context.

To begin, recordings of Jamaican popular music prior to the 1960s followed a similar format to the majority of recordings made elsewhere, namely, the live recording, and the pseudo-document. Initially, songs were recorded live with the band and vocalist, and later, with the introduction of multi-track tape machines, with the band and vocalist often recorded separately. A feature unique to Jamaica, one that would have a major influence on the way music would be produced, was the concept of the sound system, and later, sound clashes. Sound systems, essentially outdoor public address systems through which music for dancing was played, began appearing in the 1940s as an affordable alternative to live music. Turntable operators and deejays (announcers similar to radio disc jockeys) were the core personnel of sound systems, and their interactions with the crowd were a vital component of the format. The sound clash was an event in which two rival sound systems were set up and battled in front of an audience, in

17 David Katz, author of Solid Foundation: An Oral History of Reggae (2003) charts the development of Jamaican popular music with a special interest in the cultural, social and political factors, providing an excellent resource for research in this area. 
turn playing their selection of recordings (recordings often produced exclusively for specific sound systems) with the audience deciding the winner through their vocal appreciation (Stolzoff, 2000; Katz, 2003).

The sound system format would have enormous global significance and can be considered to provide the template for the block parties of New York in the 1970s, the large outdoor and warehouse parities associated with UK 'rave' culture in the 80s and countless other genres of music that rely on public address systems and playback technology as the primary form of performance. By the mid 1960s, the competitive nature of sound systems combined with a nascent focus on locally produced music resulted in the alignment of Jamaican record labels and studios with particular sound systems. Duke Reid, owner and operator of a popular and pioneering sound system Duke Reid's The Trojan in the 1950s, began recording Jamaican artists in the 1960s at established recording studios. These were for play on his sound system and for license in the UK. During the 1960s, Reid established his own studio, releasing many successful records through his label Treasure Isle Records and in 1968, he established Trojan Records, a label that focused more on reggae, and later, dub. Reid was not the only person to establish this production, marketing and distribution format. Clement Seymour "Sir Coxsone" Dodd, Reid's main rival, operated 5 different sound systems by the late 1950s, and opened the famed Studio One recording facility in 1963. This vertically integrated streamlined and successful business model, along with the fiercely competitive nature of sound systems, would have a major impact on the speed at which the style of music and its production developed (Barrow and Dalton, 1997; Katz 2003).

In 1968, Osbourne Ruddock (better known as King Tubby), a sound system engineer and mix engineer at Treasure Isle, would pioneer a new format of mixing that was designed specifically for the sound system, a format which is considered revolutionary not only in the context of Jamaican popular music, but on a global level ${ }^{18}$. The techniques he developed can be seen as producing a

18 The multiple-award-winning documentary Dub Echoes (2008), directed by Bruno Natal, follows the global impact of Ruddock's innovations. 
unique form of meta-document, one that not only directly transforms a pseudodocument into a meta-document, but also one that introduces an entirely new concept of performance. This new format evolved in two stages. The first was the creation of 'the version', which is reputed to have happened around 1968. The version, also known as 'the riddim' is essentially an instrumental rendering of the song, without the vocal. The version is said to have come about by accident, whereby a vocal track was left off the mix when the disk was cut. King Tubby was not the engineer for this cutting, but was present at the time, and immediately saw the potential of such a mistake. As Katz recounts, the impact of this recording was immediate at dances, as the vocal version was played first, and the instrumental 'version', was played next, with the crowd singing along (Katz, 2003, p. 166). The power of 'the version' to engage with the audience was apparent, and before long, King Tubby was cutting 'versions' on the B-sides of the majority of Treasure Isle releases, with other labels following the trend. The version also allowed more space for the deejay to perform at sound systems, which in turn allowed for the development of 'chanting', 'toasting', 'chatting', and 'peps', deejay vocal techniques that are regarded as precursors to hip hop MCing or 'rapping'. ${ }^{19}$ Deejays including URoy, Dennis Alcapone and King Stitt began to feature on recordings in the early 1970s. As such, the version inadvertently opened the way for new kind of recording artist. The inclusion of the deejay in Jamaican popular music recordings is an example of how important the sound system was in the development of recording practices; practices that King Tubby was actively developing at this time. The second stage involved the rebalancing of instruments for the version. King Tubby would create versions by pulling down the volume fader of the vocal track after the original mix was complete. In this process, he discovered the potential for entirely new mixes to be created. Before long King Tubby would develop the revolutionary style of mixing that he has become famous for: the dub mix (Barrow and Dalton, 1997).

Dub mixing can be described as an alternative rendering of a pseudo-document, in which the individual instruments are re-evaluated, treated, and arranged by

19 In Sonic Bodies (2011), Julian Henriques provides excellent analysis of the role of the deejay in Jamaican music. See chapter 7 (p. 175-199) 
the engineer at the mixing desk. Often, songs are stripped back to the drums and the bass, the latter usually increased in amplitude. The other elements, such as the guitars, keyboards, horns and vocals, are brought in and taken out of the mix at various times, often with extreme spatial and modulation effects. King Tubby incorporated the reverb and delay effects he used live with his sound system Tubby's Hometown $\mathrm{Hi} \mathrm{Fi}$, and augmented them with audio modulators and sweeping filters. Later, sirens, synthesisers and test-tone oscillators would also feature in dub mixes. The arrangement and effects would be performed live to a recording by Ruddock at the mixing console. The response to this style of mixing was positive, and dub mixes became very common, often on the B-Side of a 7" vinyl pressing. Other producers, notably Lee Perry (who was then affiliated with Dodd and Studio One), would adopt the style and create their own dub mixes. As David Toop writes:

\footnotetext{
Lee Perry and Augustus Pablo, in particular, were recording increasingly exotic and distinctive music during the 1970s. On albums such as Perry's Super Ape and Pablo's East of the River Nile, the mixing board becomes a pictorial instrument, establishing the illusion of a vast soundstage and then dropping instruments in and out as if they were characters in a drama. (1995, p. 117-118)
}

By way of example, I would like to examine an early dub mix by King Tubby that displays a variety of techniques that would becomes staple features of dub mixing. The track, using the established 7" format popular in Jamaica in the 1970s, is a dub version of the Jacob Miller song "Baby I Love You So" (1975), with the subtitle "King Tubby Meets the Rockers Uptown". The track begins with Carlton Barrett's drums, playing a snare drum fill on the anacrusis. Unlike the original, King Tubby has sent the drums to a tape delay, set at a speed that produces a dotted crochet iteration. Combined with the original 'one-drop' drum pattern (a simple pattern where the hi hat plays quavers, and the snare and bass drum play on beat 3), the drums and their delay form a new rhythm, which gives the high hat and snare a swung semi-quaver movement. Incidentally, this double time feel would become known as the 'rockers' feel, a style that drummers would play without tape delay, which further emphasises the role of technology in the 
development of Jamaican popular music. The bass line, louder in the mix than the vocal version, plays throughout. The drums and the bass are given the most prominence in this mix. Occasionally, the snare drum is sent to a reverb channel, giving it an exaggerated and temporary spatial dimension. This technique is a hallmark of dub mixing. The drums and bass play throughout, until they are faded out earlier than the other instruments, to allow for Miller's vocal "I love you so" to conclude the mix. Throughout the track, the guitar, piano and melodica variously interject, treated with delay and reverb effects, controlled using either a fader or the channel's mute switch. The vocal is subjected to very crude manipulations, often faded in, with delay and reverb, to be muted in mid phrase, the delay repeating the truncated words across the beat. King Tubby's performance is strong in this mix.

Dub mixing of the early 1970s created a new form of meta-document on a number of levels. At face value, these mixes were not dissimilar to the kinds of works released by The Beatles a few years earlier, as dub mixes, much like songs found on Magical Mystery Tour and The Beatles, were studio manipulations of performed material, though this is where the similarity ends. While some of the later works of the Beatles (such as "Strawberry Fields Forever") were clearly a result of studio manipulation, the meta-documents of the Beatles would never quite achieve the kind of dislocation between performance and manipulation that was presented by dub mixing. This may be attributed to the arresting power of McCartney and Lennon's voices, making demands of our attentional focus (an idea I will expand on in chapter five), and also as a result of social perception: The Beatles became synonymous with their recordings from 1966, the year they retired from performing live altogether. For example, where "Strawberry Fields Forever" is concerned, though I myself am aware of the inherent temporal distance between the recording of The Beatles (the band) and the subsequent manipulations and performances in the studio, I believe many people do not hear such a distinction, nor do I believe that it was intended, from a poietic perspective, that such a distinction be heard: this distinction is blurred in the recording as a matter of attentional focus. This is where dub mixing represents a marked change in the meta-document from a poietic and esthetic perspective. 
The performance of an engineer is the primary feature of the dub mix, and as such, the distinction between the instrumental performances and the subsequent studio manipulation is keenly felt.

Unlike the Beatles, King Tubby's releases were not composed of his own instrumental performances; that is, he did not play any of the instruments used in his dub mixes, and in many cases, neither did he engineer them. He, like countless other dub mixers, mixed the performances of other musicians. The general awareness of King Tubby's role in the production by the dance-going public was widespread and easily understood, even if the techniques remained mysterious to those unfamiliar with audio technology. By way of example, the sudden cut out of a vocal in mid-phrase, the delay that followed, and the subsequent fading in of an entire drum kit part points squarely to the engineer as arranger, and importantly, as a new kind of performer. The 'engineer-asperformer' concept finds common ground with the post-production techniques of Glenn Gould, though unlike Gould's splices, dub mixing creates a distinction between the original recording and the new performer, instating a temporal duality, whereas Gould's splice, much like the later works of The Beatles, collapses this distance by producing a total recorded performance, made up of performance and studio manipulation. With this in mind, we might begin to understand the Beatles recordings as supporting a work concept that, as Goehr might have it, is Analytical in conception: though the recordings of The Beatles are meta-documents with various temporal relationships between performance and production, these relationships are dissolved in such a work concept, whereby the phonograph is understood to unify the work conceptually.

Furthermore, the insertion of a 'performing engineer' between the recorded materials and the audience was not unique to dub mixing, Gould, or The Beatles; musique concrète had already established such a situation some 20 years earlier, but unlike musique concrète, dub mixes retained a strong connection with the source materials, whereas musique concrète, at least in its dogmatic forms, sought to divorce music from its source materials altogether (examined in detail in chapter two). The formal qualities of the song, such as tempo, rhythm, melody 
and harmony, along with the proficiency of the musicians and sonic qualities of the recording are still very important. In other words, if the original song was not a hit with the audience, a dub mix was also unlikely to be popular. There exists an important intertextual relationship between the source materials and the new dub mix that does not exist for musique concrète, a genre that seeks to remove any such relationships. In this way, though the dub mixer and their performance was understood as the primary focus of the recording, audiences still had to connect with the original materials. As such, the temporal distance between the performance of a band and the performance of an engineer is easily perceived from an esthetic perspective, further highlighting the engineer's role.

This multi-layered relationship has several implications for the meta-document. To begin, the concept of performance is augmented, and as a result, the concept of the work is also. While ideas surrounding authorship in Jamaican popular music are fascinating, not least of all because of the accepted practice of a single musical idea (such as an instrumental, or riddim) being used for multiple songs, ${ }^{20}$ I am unable to undertake an analysis of it here. In the context of this thesis, I will simply acknowledge that the majority of original recordings used in a dub mixes can be considered pseudo-documents, insofar as they are designed to grant access to a 'live' performance of an instrumental ensemble, which can then be understood as granting access to the work in various ways. At base, the dub mix affords a dual reading of the work, composed of two performances: those of the original musicians, and that of the dub engineer. Although dub engineers typically perform without (large) audiences in the privacy of their studios, their role as performers should not be understated. Dub engineers assertively position themselves between the original materials and the audience; their contortions at the mixing desk become focus of a listener's attention. Attentional focus to the engineer's manipulations results as a matter of distinction: between their performance and the original instrumental performance. For example, a drummer cannot 'fade out' in the same manner as a track fader on a console, nor can a vocalist repeat a word (with regenerative

${ }^{20}$ Steve Goodman in his book Sonic Warfare (2010, p. 161) provides an example of such a practice, whereby the instrumental to Wayne Smith's song "Under Me Sleng Teng"(1984), has be used as the basis of hundreds of subsequent songs. 
distortion) in the same way achieved by an auxiliary send from a vocal track to a tape echo; audio technology allows for these new performances, and the engineer, like any performer, improviser or composer, develops a certain style, imparting to the recording not only the sonic signatures of the technology used, but their own signatures through their creative and performative decision making. Though today such processes may be automated, even generated, by a computer, this does not change the nature of the performance from a temporal perspective. As far as a listener is concerned, these manipulations are perceived as having been performed between the band's performance and the moment of audition, regardless of who (or what) made the performance.

Temporality in the phonograph is a complex topic, and is the focus of chapters five and six, but in the context of the meta-document (dub mixes for example), the presence of the engineer make us acutely aware of the temporal dislocation between the recording and the production (and also between production and audition for some listeners) and for that reason, it should be noted that dub mixing represents a unique evolution in the listener's temporal awareness of the meta-document.

While the kinds of relationships I have described may represent a novel way of viewing the dub mix engineer and their relationship to the recorded sounds embodied in their mixes, it must be stated that the attention on the engineer (however that attention is perceived to have emerged) is significant and widespread. Steve Goodman likens the spread of dub's influence to the trajectory of a virus:

The "dub virus" relates not just to the direct influence of the dub reggae sound on other musics, but more than this, its catalysis of an abstract sound machine revolving around the studio as instrument and the migration of a number of production and playback processes. The dub virus hacked the operating system of sonic reality and imploded it into a remixological field. The dub virus, taken in these terms, is a recipe for unraveling and recombining musical codes. (2010, p. 159) 
The spread of dub and its influence on the production process in other genres of music attests to the significance and appreciation of the dub meta-document; it is not merely a theoretical consideration of a production method, but a thoroughly audible, unique and widely understood orientation of performers and engineers in the milieu of record producing. This meta-document can be found in the genres of jungle, drum'n'bass and in the vast number of remixes by many artists in many different genres, where the original audio stems are re-evaluated, processed and recombined in ways unique to the remix artist. This is the legacy of the dub meta-document. Indeed, many aspects of hip hop have roots in Jamaican music traditions (especially sound system culture and the deejay), and while the recombination of musical codes is a fundamental trait of hip hop production, the meta-document encountered in dub is not generally found in this genre of music. Instead hip-hop, inspired by the sound system, the deejay, and the technological focus of Jamaican music, established a new and unique form of meta-document.

\section{The phonographs of hip hop}

Along with these elements of Jamaican music, hip hop traces much of its heritage to the traditions embodied in African American culture, including the music of jazz and blues, and before, to pre-slave-trade musical traditions in Africa. Equally, the vocal component of hip hop (known variously as MCing and rapping) can be traced to the work songs of the south, word games (such as the Dozens) and the style of announcing that African American radio personalities developed through the 1960s. ${ }^{21}$ Perhaps the most notable catalyst for the emergence of hip-hop was Clive Campbell, also known as DJ Kool Herc, in the mid 1970s in the Bronx, New York. Campbell was born in Kingston, Jamaica in 1955, and moved to New York at the age of 12 (Toop 2000, p.19). Campbell took the sound system culture of Jamaica, the culture of versions and toasting (an emerging culture in Kingston around the time he emigrated) and adapted it for

\footnotetext{
${ }^{21}$ David Toop's book Rap Attack (2000) provides a compelling historical account of these influences, while Jeffrey O. G. Ogbar's Hip Hop Revolution: The Culture and Politics of Rap (2007) provides a deeper cultural context for the development of hip hop.
} 
the communities of the Bronx. ${ }^{22}$ Campbell is widely recognised as a pioneer in two regards. He helped establish block parties (sound systems set up outdoors, usually in the communal spaces of housing projects or basketball courts), and the turntable technique whereby the 'break' of a record is extended.

Much like the sound system culture of Kingston, Campbell helped to establish a form of entertainment that was affordable. In New York, the sounds of Jamaican popular music were not well received and so Campbell switched his selection to Latin and funk records (2000, p.60). Where Jamaican sound systems culture had grown to a point where music was being produced especially for 'the dance' (i.e., instrumental cuts and dub mixes for vocalists to 'toast' over), Latin and funk music popular in the Bronx did not have the same space for additional live vocalists. Campbell's solution was to play the 'break', a section of a recording where the arrangement focused on the drums and possibly the bass instrument. He would join the break of one record with another break from different record, thereby keeping the musical materials minimal, as these were more suitable for dancing and live vocalising. Joseph Saddler (also known as Grandmaster Flash) would later extend this technique by using the same break from a second copy of the record on a second turntable, to extend the original break by manually looping it (or 'juggling' as it became known). He modified an audio mixer with a toggle, that allowed him to instantly select which track was auditioned by the master output (2000, p.62). This innovation (which later developed into the cross-fader, a staple feature of most modern turntable mixers), allowed Saddler to cue and play a single break from 2 records for an indefinite period. This technique was largely responsible for the emergence of the MC and b-boy and bgirl dance culture. The relationship of the selector (known as the DJ in hip hop), the toaster (MC or rapper), the sound system, and the audience, was thereby successfully transplanted from Jamaica to New York by the mid 1970s, with a number of new technical and musical innovations.

\footnotetext{
22 Campbell was not alone in this regard, Lloyd Barnes, once an engineer for Duke Reid in Kingston, set up a
} popular recording studio, label and sound system in the Bronx during the 1970s. 
From the early days of hip hop in the 1970s through to the present, the production techniques associated with the genre have varied greatly, and the relationship of the music to the phonograph is equally diverse. Through the innovations of Campbell, Saddler and others, the phonograph and the turntable would earn the status of a musical instrument, just as the mixing desk had in dub music. With the introduction of digital samplers, the 'breaks' could be automatically looped and combined with other recordings. Drum machines and synthesizers were also utilised, and tracks made up entirely of electronic sources would become commonplace. The kinds of meta-documents produced under the banner of hip-hop not only extend the innovations of dub music, but they also introduce entirely new concepts.

To begin, hip-hop introduced the idea of the phonograph-as-instrument to the world of popular music, and while its discovery in this context was influenced by the unique concerns of Clive Campbell, it is not the first time the phonograph had been used in such a way. German Composer Paul Hindemith is believed to be the first composer to compose explicitly for phonographic records, with his piece Grammophonmusik from 1930. John Cage was said to be in attendance at the world premiere, and his piece Imaginary Landscapes Number 1 (1939) also employs turntables in his ensemble (Katz 2004, p. 113). Since this time, many people have used the phonograph, in all its formats, as a source for creating new music, not least of all Pierre Schaeffer and the composers of musique concrète. In more recent years, a number of sound artists have continued the tradition, including notable experimental composers Philip Jeck and Otomo Yoshihide, alongside the growing number of turntablists; musicians who often trace their practice back to the innovations of Campbell and Saddler. In a live context, the turntable is best understood as an instrument. Whatever sounds were captured by the phonograph are recontextualised by the present and engaging manipulations of performer, thus the original documentary aspect of the phonograph used is greatly diminished by the performance. That is not to say that the turntablist seeks to diminish the importance of the source materials, quite the opposite is true; the performer is often heavily invested in the 
materials, which is why they are selected for presentation. ${ }^{23}$ The intertextual relationship between source materials and their recombination in hip hop, as in dub, is a topic of great interest, but here I will simply note that performance, albeit an intertextual performance, becomes the primary focus of engagement when hearing a turntablist live. When such a performance is recorded, however, a unique form of phonograph-as-document emerges.

Much like dub music, the early recordings of a turntablist at work highlight the temporal shift between the recorded materials and the recorded performance. One such example is a 1981 recording by Grandmaster Flash titled "The Adventures of Grandmaster Flash on the Wheels of Steel" - where 'adventures' denotes his performance, and 'wheels of steels' references the turntables used. On this 7-minute recording, Saddler uses 11 different records and 3 different turntables, displaying his 'break juggling' skills, as well as other innovations, such as scratching and 'punching' in string and horn stabs, as well as individual drums. Where recordings of turntablists performing depart from the metadocument of dub-mixing is in the variety of different sources: where a dub-mixer acts as a performer of a pseudo-document, a turntablist acts as a performer of (potentially) multiple document types, thus introducing multiple temporal relationships. By way of example, a turntablist may juggle from a 1930s swing record to a modern funk record, thus combining music of different origins. ${ }^{24}$ The importance placed on the original materials of a turntablist's performances is significant within the hip hop community. The pursuit of rare or underutilised recordings in hip hop culture, as exemplified in the practice of 'crate digging' is important to many DJs, and later, producers, and the combination of these materials is a core methodology of both turntablists and composers. However it is the temporal relationship established in the combination of two records that is the most significant factor from an ontological perspective. In this sense, a turntablist mix is more curatorial than a dub-mix; that is, it is more a gathering of multiple sources than the reworking of a single source. Dub mixes by contrast

\footnotetext{
${ }^{23}$ Sophy Smith's book Hip-Hop Turntablism, Creativity and Collaboration (2013), usefully explores the motivational and creative context of turntablism in hip-hop.

${ }^{24}$ Los Angeles based hip hop ensemble Jurassic 5 recorded "Swing Set" for their album Quality Control (2000). It samples a number of different swing records, juxtaposing these samples with an Idris Muhammad funk drum break from Rusty Bryant's track "Fire Eater" (1971).
} 
are best understood as the product of a performed deconstruction of a singular entity, be they a live performance or a simulation of one.

The complex temporal relationships evident above become more complicated with the introduction of the digital sampler. Drum machines with sampling capacity were available from around 1984 with the Linn 900 (developed by engineer Roger Linn). Other drum machines including Roland's TR-808 also utilised sampling technology, but the devices I am most concerned with in the context of enhanced temporal relationships are those that were designed to allow users to record their own samples. The most popular models used in hip hop, the Emu SP12 (and later, the SP1200), and Roger Linn's MPC 60 (developed for Akai), came onto the market in 1987 and 1988 respectively. This allowed for music producers to achieve the effect of looping breaks without the labour intensive and highly skilled techniques associated with turntablism. It also allowed for multiple recordings to be layered to an extent that could not have been as easily achieved in previous years. The productions of The Bomb Squad, who were responsible for a number of albums by Public Enemy, including their 1987 debut Yo! Bum Rush the Show, exemplified this technique. Bomb Squad producer Hank Shocklee recounts his experiences with Public Enemy:

We took whatever was annoying, threw it into a pot, and that's how we came out with this group", Shocklee told Keyboard magazine, "We believed that music is nothing but organized noise. You can take anything - street sounds, us talking, whatever you want - and make it music by organizing it. (Shocklee in Toop, 1995, p. 123)

Hank Shocklee here exudes a notably Cageian philosophy towards sound and music, one that is more commonly associated with the post-modern aesthetics of $20^{\text {th }}$ century western art music than hip hop. It may be argued that heavilylayered hip hop of this type finds connections with the audio montages of musique concrète, or even Cage's own Rozart Mix (1965), but unlike these earlier works, hip hop employs another layer that differentiates it from such pieces. This layer is the performance of the $\mathrm{MC}(\mathrm{s})$, and it functions in a way similar to that of the pseudo-document: it arrests the attention of the listener, and thus 
establishes a relationship between the listener and the document that is not found in 'the work' focused phonographs of western art music. For this reason, hip hop recordings of this nature represent an interesting kind of phonographic document. On the one hand, the instrumental aspect of the music displays the curatorial meta-document features described above, yet when combined with a vocal performance, our reading of the phonograph can change substantially: despite acknowledging that a track is composed of multiple sources, the arresting power of the vocal homogenises the discrete performances that make up the instrumental into a singular temporal event that has the singular function of 'vocal accompaniment'. In this way, the meta-document of hip-hop assumes a pseudo-document veneer. I have alluded to the power of the human voice to arrest attentional focus previously in this chapter in a discussion of The Beatles and their later recordings, and though I will explore this concept further in chapters five and six, I will here briefly introduce the basic concept.

\section{The human voice and the meta-document}

In Listening and Voice (2007), Don Ihde considers the esthetic process of listening to instrumental music; that is, music without vocal performances. He writes:

In all music, sound draws attention to itself. This is particularly the case in wordless music, music that is not sung. Here the "meaning" does not lurk elsewhere, but it is in the sounding of the music. There is even a sense in which that the listening that music calls for is a different listening than that called for by word. Wordless music, in its sonorous incarnation, when compared to language is "opaque," as nothing is shown through the music. The music presents itself; it is a dense embodied presence. (Ihde 2007, p. 155)

In my view, the opacity of instrumental music in recordings, to use Ihde's term, allows for the temporal relationship between performance and production to be more easily identified. Indeed, a great number of different aspects of the recording may be attended to in a recording without vocals, depending on the 
intentionality of the listener, as the ear is not concerned with interpreting 'meaning'; a 'different listening' is afforded. In recordings where a vocal is present, listeners are immediately confronted with a 'meaning' not carried in the music alone, and they are drawn to it. This is not only a matter of interpreting language, though this is a common feature. In Ihde's view, listeners also undertake an unconscious physiological appraisal of the vocalist and the environment within which they vocalise:

The wheezing voice of the emphysemiac, of the too-far-along smoker, bespeaks the interior state of the body and its pathology... the spatial significations of where, of direction, and of surroundings are also sounding. $(2007$, p. 195) 25

In recordings where music and vocals coexist, the listener's attentional focus (a topic covered extensively in chapter five) is captured by the voice, the instrumental music moves to the background of this focus, and the complexity of the meta-document becomes harder to attend to. As I will also discuss in chapter five, this phenomenon presents itself in all recordings where a voice is present. It should be noted that the human voice does not hold the power to arrest attention exclusively. For example, the surprisingly loud saxophone solos of Stan Getz from Getz/Gilberto (1964) can arrest listener attention as much as João Gilberto's vocals. However the human voice arrests listener attention with a consistency that eludes other instruments, primarily, as Ihde suggests, as a result of a listener's search for the meaning of the words recited and their assessment of the physiological state of the vocalist. Though in some instances the homogenising effect of multiple voices can lead to a more relaxed attentional focus (such may be the case with choral music), this basic listener engagement with the human voice persists, and with greater consistency and specificity than encountered with instrumental sounds.

Returning now to the diverse temporal relationships found in the metadocument, I would like to conclude this chapter by highlighting a particularly

25 Steven Connor makes a similar assessment of the voice in the opening pages of Dumbstruck (2000, p. 3). 
interesting and increasingly popular form of phonograph; producers of which recognise the impact of technology on a listener's experience of temporality. These meta-document types might include recordings made to sound as though they were recorded in an earlier era, often through the use of period-specific recording equipment, and yet we are aware that they are not of that time. Portishead's album Dummy (1994), The Cinematic Orchestra's album Motion (1999) and Lord Finesse's album The SP1200 Project: A Re-Awakening (2014), each employ music technology of earlier decades to impart specific sonic signatures to their music. They also sample music of earlier periods to heighten this effect. The result of this methodology can be pronounced: in the case of Motion and The SP1200 Project, one might be led to believe that these albums were in fact recorded in the 1960s and 1990s respectively, though they may also present telltale signatures of their actual production dates to an attentive listener. In this way, these meta-documents simulate a temporal distance between the moment(s) of capture and the moment of audition. The potential engagements with a phonograph's sonic signatures and the temporal displacement between the past and present such technology suggests are the topic of chapter six, but here I will note that the myriad of production techniques available to an engineer may augment the meta-document in a number of interesting ways, whereby the use of technology can expand the relationships between the past, the present, authorship and performance enormously.

It is important to note here that the range of possible meta-document types is extensive, and worthy of further research. I have highlighted a particular class of meta-documents, ones in which the temporal disparities of source materials are apparent, and the notion of performance (and authorship) is extended into the production of the phonograph. I have explored these particular meta-documents as they are of personal interest to me, and because they are relevant to focal points of this thesis, particularly environmental sound composition (see chapter four). It is my belief that further exploration of meta-document types is useful given the ever-expanding production methodologies employed by recording artists. As observed in the 1980s, the introduction of turntables and samplers greatly expanded the meta-documents of hip hop. Given the current 
diversification of cheap music software types and the rate at which this diversification is occurring, it seems inevitable that the meta-document will also continue to diversify. ${ }^{26}$

Before embarking on a discussion of the phonograph-as-work in electroacoustic composition, a discussion that is central to the next chapter, I would like to reiterate the central theme of this chapter. Though several types of documents have been discussed, all phonographs exhibit two core features, detected in the relationship between the past and the present. Of this relationship in the photograph, Barthes muses 'the thing of the past, by its immediate radiations (its luminances), has really touched the surface which in its turn my gaze will touch' (1981, p. 81). Conceived in this way, the photograph is an object 'touched' by both the past and present. In a similar way, the phonograph too is 'touched' by both realities, and indeed where microphones are used it also 'touches' both realities through the movement of diaphragms. The documentary power of the phonograph is found in this immutable relationship. The second essential feature of the phonograph is that it is abstracted from a past reality: the process of committing sound to the phonograph results in a distinction between the phonograph and the materials it captures. Those creating these recordings thereby compose phonographs, though they may not acknowledge their authorial role, especially in situations where the engineer is thought to document a composer's work. What is revealed by the live recording, the pseudo-document and the various forms of the meta-document, is that the specificity of the recording's production, especially the use of splicing, overdubbing, the inclusion of the human voice and the level to which the sonic signatures of technology are heard, plays a vital role in the way in which documentary and abstraction are perceived in the phonograph. In making these distinctions of phonograph types, we can begin to discuss the various roles of those participating in the phonograph, be they composers, performers, engineers or listeners, in ways that are mindful of these core features. As I explore throughout this thesis,

\footnotetext{
26 Matthew Homer's paper "Beyond the Studio: The Impact of Home Recording Technologies on Music Creation and Consumption" (2009) provides some interesting accounts of the perceived relationships between home recording and innovations in music production, while Allan Watson's Cultural Production in and Beyond the Recording Studio (2015) provides more in-depth analysis.
} 
understanding phonographs as document types also enables phonographs produced within differing cultural contexts to be examined in similar terms, thus revealing commonalities between the recordings of seemingly disparate genres. It is my hope that in opening a dialogue between different musical and recording genres that the parameters of these genres might be expanded. 


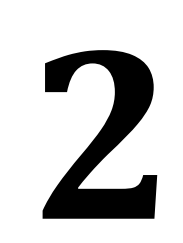

\section{The Phonograph-as-Work}

As discussed in chapter one, the phonograph-as-work is defined as a recording in which its inherent 'documentary' capacity is eclipsed by its other essential feature: its ontological distinction from the source materials. This phonograph type can be found in many genres of music, including certain types of electronic dance music and ambient music, though composers within these genres do not necessarily conceive of their recordings in these terms. The genre of music within which an understanding of the phonograph-as-work is most actively promoted is electroacoustic composition. This genre constitutes the core genre within which an exploration of the phonograph-as-work will be unfolded throughout this chapter. There are a number of reasons why I have chosen to explore the phonograph-as-work within electroacoustic composition. In addition to the emphasis on such a reading (among others) within the genre, electroacoustic composition extends upon many of the ideas discussed in chapter one, specifically the relationship between the nominalist work and recording practices within western art music. Electroacoustic composition also has a direct relationship with environmental sound composition (discussed in chapters three and four), and as such, an exploration of electroacoustic composition here serves to contextualise themes explored later in this thesis.

To begin, it should be noted that the phonograph-as-work is not the only kind of phonograph type found within electroacoustic composition. In fact, though many composers may promote their compositions as 'phonograph-specific', in many 
instances electroacoustic composition presents variations of the meta-document type discussed in chapter one. In fact, only a very particular combination of materials and their arrangement result in a phonograph-as-work, a combination I will explore later in this chapter with regard to Simon Emmerson's ideas as presented in "The Relation of Language to Materials" (1986, p. 17-39). Though the phonograph-as-work may be not a prevalent reading, the pursuit of this phonograph type within electroacoustic composition and the relationships revealed between the composer, the work and the phonograph in this pursuit, provides a perspective on electroacoustic music and the phonograph which has a number of interesting facets.

Significantly, it must also be said that while a great deal of energy has been dedicated to the analysis of electroacoustic composition, the phonograph itself and its role in the appreciation and evaluation of electroacoustic works has been largely unaccounted for. As I will detail, where early forms of electroacoustic composition are concerned, the phonograph was generally perceived as a new medium for delivering two well-established aesthetic concerns of western art music. The first of these concerns is the nominalist conception of the work: early electroacoustic works sought to continue this conception, despite the challenges the phonograph presented to this idea. The second concern is that of absolute music; whereby electroacoustic music composers sought to mute the source context of their recordings in order to promote the work as self-referential. As I will show, both of these aesthetic concerns did not translate easily from instrumental composition to electroacoustic composition, yet transfer they did, and the influence is still widely felt within the genre. The tension surrounding these ideological and material concerns will be explored here and also in chapter four. While electroacoustic composition may have been considered radically different from instrumental composition in terms of its materiality, there can be little doubt that the dominant aesthetic concern of absolute music particularly was successfully adopted during the mid-twentieth century from the instrumental world to the new electronic format despite the challenges, as exemplified in the theory and practice of musique concrète and Elektronische 
Musik. As I will also discuss, the entrenched disdain for mimetic materials ${ }^{27}$ in western art music, those that reference cultural or natural sounds, was also transferred into the new electronic form at this time. ${ }^{28}$ While the success of these genres in delivering absolute music was the primary concern of early composers of electronic works, the most fundamentally new aspect of electronic music, the phonograph itself, remained largely unexamined. In many respects, the phonograph continues to be seen as a new but somehow neutral delivery format for an existing set of compositional values. The question of how the phonograph affects the importance and appreciation of these concerns is yet to be addressed. This question constitutes the central concern of this chapter. Before proceeding, it is important to provide some historical context to the genre of electroacoustic music, in order to discover how it inherited its dominant traits.

\section{Early electroacoustic music}

A substantial body of analytical and theoretical work has been produced on the subject of electroacoustic music. It is not my intention to survey the entire field, nor to provide a review of the available literature on the subject. Rather, I will be assessing the core threads of theory and practice that relate to the role of the phonograph within the genre. To begin with, I will examine the relevant aspects of Elektronische Musik and musique concrète, genres that have had the greatest impact on electroacoustic composition, before focusing on the ideas of electroacoustic music theorist Simon Emmerson.

The roots of electroacoustic composition begin long before the well-documented practices of the late 1940s and early 1950s in Paris and Cologne. Douglas Kahn, in his book Noise Water Meat: A History of Sound in the Arts (1999) dedicates a chapter to the under-examined period between Russolo's path breaking manifesto The Art of Noises (1913) and the early experiments of Pierre Schaeffer's musique concrète, with special consideration given to early

\footnotetext{
27 As Smalley has it, 'mimesis in music is the conscious and unconscious imitation or representation of aspects of nature and culture' (1996, p. 84). See also Emmerson, S. (1986).

${ }^{28}$ Pierre Boulez' comments surrounding the works of musique concrète in Notes of an Apprentice (1968) are exemplary of this disdain.
} 
radiophonic pieces and Russian revolutionary film. This period includes some very important works, not least of all Walter Ruttmann's radiophonic portrait of Berlin in Wochenende (1930) (a piece I will examine in chapter three with regard to environmental sound composition) and Toch and Hindemith's incorporation of gramophones into the concert hall setting in Grammophonmusik (1930), cited in chapter one. I will begin my discussion with the post-WWII practices of musique concrète in Paris, and Elektronische Musik in Cologne, as these two practices not only spawned a great deal of music, but they also gave rise to strong aesthetic positions and methodologies that are essential to the emergence of electroacoustic composition. While the inception of Elektronische Musik occurred after the first works of musique concrète, I would like to discuss Elektronische Musik in the first instance, as the practice affords means to the discussion of the nominalist work and the phonograph initiated in the first chapter.

\section{Elektronische Musik}

Elektronische Musik is closely associated with the Nordwestdeutscher Rundfunk (Northwest German Broadcasting) studios in Cologne founded in 1953 under the direction of Herbert Eimert. The practices and methodologies of the genre are well documented by Eimert (1957), and while its emergence marked the beginning of an altogether new exploration of western art music's materiality through electronic synthesis, it is not the novel aspects of Elektronische Musik I am concerned with. Rather, I will focus on the established ideas, the tenacity of absolute music and the enduring nominalist composer/work relationship in particular as they contribute the most to the status of the phonograph in electroacoustic composition.

There can be little doubt that Elektronische Musik was understood, above all, to expand the repertoire of western art music. As Eimert writes in 1957:

There is an essential relationship between electronic music and the traditional world of sound, not only the fact that musical elements are 
defined by pitch, duration and intensity, but also because of the connection between it and the most contemporary development of musical thought. Electronic music is, and remains part of our music and is a great deal more than mere 'technology'. But the fact that it cannot be expected either to take over or to imitate the functions of traditional music is clearly shown by the unequivocal difference of its material from that of traditional music. We prefer to see its possibilities as the potentialities of sound itself. No position such as this could be reached by a mere transference of the traditional into the electro-acoustical. New ways of generating sound stipulate new compositional ideas, these may only be derived from sound itself which in its turn must be derived from the general 'material'. (Eimert, 1957)

Eimert here provides a very clear overview of Elektronische Musik's foundational principles. It is his desire for a reflexive relationship between the electronic materials and compositional ideas that finds common ground with musique concrète, thus helping to pave the way for electroacoustic composition as a convergence of the two genres. However the most important aspect of Eimert's outlook relates to the merging of pre-existing notions of musical structure in western art music with the new electronic materials.

Where Eimert notes that there is a relationship between electronic music and 'the most contemporary development in musical thought', we might take Eimert's 'development' to mean the incorporation of Serialism into the language of western art music. Indeed, in the same article, Eimert declares it is certain that no means of musical control could have been established over electronic material had it not been for the revolutionary thought of Anton Webern. Nevertheless, the compositional equipment of electronic music must be more than an extension of twelve-tone technique' (1957, p. 1-10). As Eimert suggests, though he saw great potential for Elektronische Musik beyond Serialism, he conceived of Serialism as bringing musical structure to the world of synthesis. It is this same idea that positions the serial-governed works of the genre as nominalist in form: the structure of the composition exists independently of the phonograph, with the composer acting as a mediator between the work and its actualisation. While a full account of Serialism and its incorporation into Elektronische Musik falls beyond the remit of this thesis, there can be little doubt 
that there was compatibility between the two as Eimert alluded to and as Stockhausen was quick to explore. The appeal is easily understood;

Elektronische Musik provided a material and technological revolution to pair with the structural revolution found in Serialism. The marriage of Serialism and electronic synthesis was already a reality by 1957: Karlheinz Stockhausen's Studie II (1954) is exemplary, as I will later detail.

While Serialism was by no means the only tool used in the composition of Elektronische Musik, it does reflect a prevailing attitude of early practitioners. For composers, works of Elektronische Musik did not exist on record, but rather in a more traditional nominalist form. As Lydia Goehr might have it, the recording of an Elektronische Musik work holds the same function as the score or a performance in relation to the work where pre-composition is present; that is, the phonograph is a physical actualisation of the abstract work. In other words, for composers of Elektronische Musik the phonograph itself had little or no theoretical impact on the accepted status of the musical work, though as I will discuss, its challenge to this established reading did not go unnoticed.

\section{Studie II (1954)}

That a score of Studie II was produced provides a number of insights into Stockhausen's attitude towards his own work and the phonograph he created. To begin with, and given that Stockhausen made detailed plans of Studie II before working with sound in the studio, ${ }^{29}$ his realisation of the work is in some ways a performance of a score; Stockhausen would have to manipulate the synthesizer and tape machine in such a way as to accurately reproduce his markings, which was no small undertaking in $1954 .^{30}$ If Stockhausen believed that his recording of Studie II was in fact the definitive work, then it stands that the score would have served its purpose upon the recording's completion, and it might be assumed that the score could have been filed away without publication. The

\footnotetext{
${ }^{29}$ Stockhausen created a number of detailed worksheets that were to serve as plans for the realization of Studie II. They are currently held at the Archiv der Stockhausen-Stiftung für Musik in Kürten.

${ }^{30}$ Given that the pitch, amplitude envelopes and dynamics of the piece were serially governed, it is almost certain that the score of Studie II was produced before the recording.
} 
score of Studie II was in fact published by Universal Edition Vienna in 1954. For Stockhausen, there may have been a strong technical consideration contributing to its publication, namely the rapid deterioration of early magnetic tapes. In his preface to Sirius (1977) Stockhausen writes:

I have not yet lost all hope that a short time before I passively watch the total deterioration of my electronic tapes, someone will invent a cunning device which will translate them into indestructible recordings. (Stockhausen in Tannenbaum, 1987, p. 22)

Stockhausen's desire for indestructible recordings would be realised (in part) by the introduction of digital recording, given the medium's ability to accurately reproduce the same information without the degradation associated with tape. ${ }^{31}$ However, for Stockhausen the score was not simply a safeguard against the loss of recordings. When asked by Mya Tannenbaum in 1980 if the reason for producing scores of his electronic works was to increase the size of the audience 'so that from the transcriptions, anyone - not just Stockhausen - will possess the key to performing part of the music of Stockhausen?' Stockhausen replied: "in theory, it's like that. In practice, no" (1987, p. 21). In practice, Stockhausen believed that the limitations of the score to produce the exact result meant that recreations of his works fell short. He recounts such a remake at the University of Stockholm in the 1970s of Studie II: the phonograph was remade "according to the instructions published in my score, but without my collaboration...it was awful" (p. 22).

Stockhausen's comment reveals a strong affinity with the ideas of Stravinsky regarding the recording of the latter composer's orchestral works. As discussed in chapter one, Stravinsky was moved to record his entire repertoire of compositions so that they might stand as the definitive interpretations of his

\footnotetext{
31 While digital recording has a clear advantage over analog tape insofar as it does not suffer from the same kind of degradation associated with repeated plays and atmospheric conditions, it does suffer from constant changes in digital storage formats, software changes, changes in digital system interconnectivity and so on. If we assert that an audio file must be reproduced in order to hold value, then a digital file is no less 'indestructible' than an analog recording.
} 
work. Stockhausen articulates a similar disposition regarding his electronic works:

\footnotetext{
I hope that a system of conversion [lossless audio archiving] is completed as soon as possible, one that can hand down to the future both my works and my personally recorded interpretations. No one can imagine how close to my heart the interpretations are. I don't say I am the absolute holder of the secrets of my music, and I don't consider myself their interpreter by right, the only person qualified to perform a work, like Momente, let's say. But I have indicated through my interpretations the qualitative goal to aim for in the light of my example. (1987, p. 26)
}

Stockhausen here exhibits just how strong the nominalist relationship between himself and his electronic works is, and as a matter of a composer's intention, Stockhausen's position is understandable. Inspiration aside and despite the obvious technological and material differences, he composed Studie II in much the same way he composed other pieces of instrumental music. However, from the perspective of a listener, it could be argued that an altogether different understanding of the work emerges.

At first, we might assume that a 'dual reading' among listeners is common. As the piece has many similarities to instrumental music, especially with the inclusion of atonal contrapuntal lines, the piece, as Stockhausen intended, may be understood as authored by the composer and existing independently of the recording (or indeed the score). We might assume that this is how Stockhausen himself initially understood his piece upon audition. However, to a listener, the work may become intrinsically related to the recording. As Mark Katz (2004) would have it, an individual may even come to associate the work with their particular copy of the recording. It is entirely possible, even likely, that a listener of the University of Stockholm's rendition of Studie II would find it, as Stockhausen did, inferior to the original recording, but for an entirely different reason. For a listener, the rendition might lack fidelity to the original recording, itself perceived as the work. While Stockhausen might feel that the Stockholm recording lacked fidelity to his theoretical conception of the work, he does 
acknowledge the role of performance and technology in the divide between the two, for the original recording of Studie II carries both the nuances of the composer's performance and the signatures of the technology used. As noted above, Stockhausen concedes the score allows others to remake his works in theory', but not in practice. He recounts:

It's practically impossible to put into writing how to decrease the volume of a loudspeaker in the short duration of a sound, unrepeatable by anyone, but easily managed by me. Not just one single time. I manage it five or ten times at a go, by manipulating, without any particular force at all, the control which is sensitive to my every touch. These are things that require the intervention of a true musician. So it's unthinkable that others may perform my works as I do. Do you know anyone who is capable of realizing my intuitions? Someone capable of using the synthesizer like me, at the controls of Sirius? (1987, p. 21-22)

It is here that the true paradox of the nominalist conception of the electronic work is revealed. While Stockhausen wishes his piece to be considered much like his instrumental works, the 'practice' of realising electronic music confuses this. As a creator of Elektronische Musik, Stockhausen is not just the composer. He is also a 'true musician' with a unique 'intuition'. However, he is not a musician in the same sense as an instrumentalist performing the work of a composer, or even their own work; as a performer of technology, in which the only possible appreciation of the performance is through the phonograph, Stockhausen melds the performance, the technology and the phonograph together into one entity. In this way, his performance does not translate the work through the phonograph, but rather he transfers the work to the phonograph.

With this point in mind the exact nature of Elektronische Musik's 'dual reading' is revealed. In the case of Studie II, the work is nominalist in theory, but phonograph-specific in practice. From this understanding a significant question arises: when confronted with the phonograph-specific reading of the work, what weight, if any, has the theoretical reading? There can be little doubt that 
the nominalist conception of the work is important for certain composers (and listeners), but I assert that once the materials are committed to tape, a new, immutable work is born. Unlike the nominalist (abstract) work, the new phonographic (concrete) work is accessible by all who encounter it. Stripped of the creative context that a nominalist reading requires, a listener is left with the most basic and understandable reading of the work: its unified identity with the phonograph. It might follow then, that despite the composer's intentions, Studie II is a true, self-referencing document: the phonograph-as-work in its truest form. However, the phonograph's essential documentary power, though eclipsed, is not absent. A trace persists: Stockhausen's performance at the synthesizer in 1954 instates a temporal distance between the time the recording was made and the moment of audition. When listening to Studie II, a listener may be aware of this past reality, which may in turn allow them to situate the work in 1954. This in itself does not separate the work from the phonograph, but recourse to this trace may begin a process of separation for some listeners. For those with knowledge of Stockhausen's compositional methods, the documentary power of the phonograph may allow them to perceive the work as existing beyond this time and beyond the phonograph itself; the reverence of the composer and their genius remains for many listeners, and the nominalist work persists: some listeners may hear 'the work' just as Stockhausen himself envisioned it. Considering this possibility, we can assert that while some may hear a nominalist work when listening to Studie II, all will hear, or at very least are able to hear, the concrete, phonograph-specific work.

My personal tendency to mute the nominalist work in favour of its concrete form reveals a position that Goehr might identify as an analytical approach: the work does not exist in ideal terms, only in its sounding. It is from this position that we can consider the nature of the sonic signatures in a reading of Studie II. Stockhausen, against his own theoretical conception of his piece, conceded that the rendition of Studie II at Stockholm University was an inferior one, for it was done, as Stockhausen asserts, without his collaboration. Perhaps it is possible that Stockhausen, against his theoretical standpoint, was invested more in his 
own 'interpretation' than he was in his own work concept and for that reason, any rendition would be inferior. As quoted above, Stockhausen believed it impossible to describe the precise nuances of Studie II, yet these nuances were 'easily managed' by his hand. It is also possible that Stockhausen was invested in the sound of his rendition, not just the performance. In my view, another recording of the work cannot possibly contain the vital ingredients found in my appreciation of the piece: the unique ingredients that constitute the sonic signatures of the recording.

The recording I own of Studie II is a digital version; a 16bit, $44.1 \mathrm{kHz}$ audio file, mastered in 1991 for the CD Elektronische Musik 1952-1960. What strikes me most about this recording, regardless of which playback system I employ, is the tape saturation of louder sounds, and the distortion that sometimes accompanies it. My engagement with the piece is technologically focused, as is my engagement with much electronic music. The particular tone of the oscillators, itself an amalgamation of the tone generator used and the tape machine that captured it is specific to this recording alone. I am also struck by its capacity to create feelings of nostalgia or rather, a capacity to stimulate my personal, dislocated experience of 1950s electronic music, not just the German variety, but also much of the electronic music created at that time. To my ears, the audio quality of Elektronische Musik is not too dissimilar to that of musique concrète, it has a lower noise floor perhaps, but tonally and spatially similar; mono recordings and the comparatively limited frequency response of tape machines in the 1950s have a certain homogenising sonic effect that is readily perceptible some 60 years later. I would like to point out that though I prefer to listen to Studie II on my studio monitors, I am still able to engage with the work's features described above; that is, despite variations in playback format, I can still focus my attention to the sonic signatures of 1950s recording technology. This is an idea I will discuss in chapter six. As I will also discuss at length in chapter six, the ability for a recording to engage with a listener's memory through its sonic signatures constitutes one of the most engaging and unique features of the phonograph. 


\section{Musique Concrète}

Musique concrète, a genre of electronic music developed by Pierre Schaeffer in the 1940s at the Paris studio Club d'Essai de la Radiodiffusion-Télévision Française (RTF), was very influential in the establishment and development of other studios around Europe in the early 1950s. Indeed, many composers had visited and created works in Schaffer's studio before relocating to other facilities. ${ }^{32}$ Though musique concrète and Elektronische Musik in particular were considered to be very different genres, largely as a result of the materials used for composition, they shared a great deal in common. As I later make clear, both genres attempted to uphold the concerns of absolute music, though musique concrète struggled to do so on account of the materials it chose for composition. Unlike Elektronische Musik, whose materials were predominantly synthesised, musique concrète used found sound. As Reginald Brindle Smith has it, whereas musique concrète

\footnotetext{
begins with prepared sound material, which is molded into its final form by a process of experimentation, trial and error, perhaps following unexpected paths to goals that were never forseen initially, electronic music [at the Cologne Studios] was composed like traditional music, first being conceived in the mind of the composer, then written down, and finally realized in sound. (Smith Brindle in LaBelle, 2006, p. 28-29)
}

The differences in methodology say a great deal about the respective genre's understanding of the work and the influence of the phonograph in this understanding. With regard to Schaeffer, much can be made of his use of the word concrète in the genre's title: the works of musique concrète were not considered abstract like their Elektronische Musik counterparts, waiting to be realised through performance, rather they contained concrete, tangible materials, forged with physical manipulations of machines, the results of which were also tangible and could be played and replayed at will. As Smith Brindle recounts, composition and technological engagement were linked processes

\footnotetext{
32 Stockhausen, before creating Studie I and Studie II in Cologne, composed Konkrete Etüde (1952) at the RTF, moving to the NWDR studio when it launched a few months later.
} 
within musique concrète and in many respects, this marriage not only acknowledged the phonograph's implicit modification of the entrenched work paradigm, but it also represents arguably one of the most significant developments in the compositional methodology of western art music in the $20^{\text {th }}$ century. Elektronische Musik can also be seen to link technology and composition in this way. Indeed I argued in the previous section that Stockhausen's rendition of Studie II is the definitive work based on his superlative performance through technology and the inferiority of subsequent renditions. However, Stockhausen in particular conceives of technology as a way to actualise the work, whereas musique concrète understands the work as bound to the technological process: as Goehr might have it, from a theoretical perspective, works of Elektronische Musik are nominalist, whereas works of musique concrète are analytical.

Schaeffer, however, was more concerned with the specific nature of the materials used and how they could be transformed and arranged, that is to say abstracted, to satisfy his preconception of 'music', than he was with the implications of his work in terms the phonograph or his significant (and influential) extension of compositional methodology more generally. In order for works of musique concrète to be considered musical, Schaeffer went to great lengths to not only acoustically strip materials of contextual referents through truncating audio, but to also cognitively strip these referents through advanced listening techniques. In prescribing ecouté reduite (reduced listening), Schaeffer attempted to re-educate his listeners in order to assist in the amalgamation of musique concrète into absolute music. Schaeffer thus attempted, unsuccessfully some have argued, to continue western art music's rejection of mimetic materials - those with cultural, non-musical signification - by transforming found sound into abstract sound objects. Schaffer's works Cinq Etudes de Bruits (1948) represents the first fruits of these manipulations, which includes such techniques as altering the speed of playback, sillion fermé (the looping of a single sound on a record) and clouche coupée (the removal of a sound's attack transient). Much has been written about musique concrète and its practitioners, not least of all by Schaeffer himself (1966/2012) and his former student Michel 
Chion (2009), and while there is a wealth of fascinating ideas presented by musique concrète, including the notions of reduced listening and l'objet sonore (ideas I will return to later) I will focus more specifically here on the methods of production.

\section{Cinq Etudes de Bruits (1948)}

Etude Aux Chemins De Fer, the first of five studies by Schaeffer, is a 2 minute and 52 second piece, composed of recordings made at the Batignolles train station, cut together in an episodic montage, the sections of which are punctuated by loud whistles and brief silences. The primary device used is sillion fermé; recordings of trains on tracks are looped and repeated to extrapolate and extend their rhythmic properties. In 1952, Schaeffer recounted his procedure in his book $A$ la reserche d'une musique concrète:

Distinguishing an element (hearing it in itself, for its texture, its color). Repeating it. Repeat the same sound fragment twice: there is no longer event, but music. (Schaeffer, 2012, p. 13)

Etude Aux Chemins De Fer is clearly concerned with the transformation of found sound into music via a focus on rhythm and while this piece is successful in deriving a new, musical context, it does not discard the original context of the trains at Batignolles. Schaeffer would address this idea in later studies, such as Etude Violette, the third of his five studies, composed the same year using recordings of a piano played by Pierre Boulez. In this study, Schaeffer again uses sillion fermé as a core device, though also makes greater use of clouche coupée; the attack transients of the piano are cut of to further disguise the source materials. Schaeffer also reverses the piano in a central section which effectively inverts the natural envelope of the piano. This is a fairly significant technique, as the temporal inversion of sound, a unique achievement of the phonograph, finds no analogous experience in the natural world. Despite the radical manipulation of materials, there can be no doubt that the source materials derive from the piano, though it is fair to say that in 1948 the techniques used in the production 
of the piece would have been far more arresting to a listener than they are today, and the recognition of source material may have been overshadowed to some extent by these techniques. Schaeffer may have been aware that his experiments were not achieving the level of abstraction he envisaged. Regarding his train recordings, Schaffer wrote:

It's exciting, but is it music? Isn't the noise of buffers first and foremost anecdotal, and thus antimusical? If this is so, then there's no hope and my research is absurd. (Schaeffer 2012, p. 12)

And Boulez, a strong critic of musique concrète, wrote in 1966:

an agreeable nonchalance made the concrete-music studio a flea market of sounds; the bric-a-brac, alas revealed no hidden treasures. (Boulez, 1968, p. 290)

What both Boulez and Schaeffer intimate in these two quotes, is that in order for the materials of musique concrète to hold any musical value, recourse to their original context must be removed. If this context survived, the materials presented a 'flea market' of 'anecdotal' sounds. That Schaeffer describes this condition as 'antimusical' reveals just how entrenched the values of absolute music were in his thinking. ${ }^{33}$

\section{Reduced listening}

Perhaps in response to the tenacity of mimetic materials in his works, Schaeffer developed the notion of reduced listening (l'écoute réduite). Reduced listening requires listeners to cognitively bracket sounds for the purposes of hearing the sounds themselves. As Michel Chion writes in Guide Des Objets Sonores: Pierre Schaeffer Et La Recherche Musicale:

33 'Absolute music' denotes music that is entirely self-referential. See Chua, D. (1999). 
Reduced listening is the listening attitude which consists in listening to the sound for its own sake, as a sound object, by removing its real or supposed source and the meaning it may convey. (Chion, 2009, p. 30)

In essence, Schaeffer's cognitive bracketing, a maneuver indebted to the Husserlian epoché, (a concept I will address in chapter three), is designed to further assist musique concrète in its creation of absolute music. That such a technique is required suggests two things. The first, as Boulez suggests, is that the techniques of musique concrète's production were not sufficient to obscure the source context. This may be said of earlier works, though it can also be argued that technology simply had not reached a sufficient level to allow Schaeffer to achieve his aims at that time. Schaeffer expressed some dissatisfaction with the audio quality of his recorders:

There is, alas, a lot of background noise with these sounds, for it has to be admitted that all these manipulations ultimately do an enormous amount of damage to the sound quality, despite all the efforts of my colleague Jacques Poullin, a sound engineer. (2012, p. 16)

The second notion suggested by the promotion of reduced listening is that regardless of a composer's best efforts, a listener may be able to deduce the source. In many respects, reduced listening acknowledges and seeks to close the gap between intention and reception in works of acousmatic music. Boulez was not alone in his observation of musique concrète's shortcomings. Schaeffer himself would admit to Tim Hodgkinson in 1986 that:

Each time I was to experience the disappointment of not arriving at music. I couldn't get to music - what I call music. I think of myself as an explorer struggling to find a way through the far north, but I wasn't finding a way through. (Schaeffer in Hodgkinson, 1986)

In this statement, like his musings on the anecdotal as 'antimusical', Schaeffer reveals how ingrained the concept of absolute music was in his understanding of 'music'. His statement also betrays the level of his desire to make musique concrète fit this definition, apparent not least of all in his disappointment. 
Musique concrète can be seen as an incredible triumph in some respects, and a failure in others. There can be little doubt that the methodological approach to composition was not only revolutionary, but it also tacitly acknowledged a fundamental reality of recording technology in a way that Elektronische Musik did not: the phonograph embodied the work itself. In my opinion, the failure of musique concrète was not that it was largely unable to arrive at absolute music as Schaeffer and Boulez suggest, but that it attempted to do so at all. That is not to say that the tools used in this pursuit of absolute music were inconsequential, on the contrary, reduced listening, the sonic object and the technical manipulations pioneered have given rise to a wealth of fantastic and successful works and ideas.

In fact, while Boulez may hear a paucity of 'hidden treasures' in musique concrète, this is by no means the dominant response. To my ears, musique concrète retains a feeling of hands-on improvisation, a kind of physical exploration of sound that reveals the human agency of its production. My engagement is not one primarily concerned with the type of materials used and their formal arrangement, but rather an appreciation of the phonograph type in attendance, and the role of the composer in its production. In many respects, works of musique concrète have much in common with the meta-documents of dub music, especially where the source materials are "unsuccessfully" obscured, and the studio manipulations are prominent. By way of example, in Cinq Etudes de Bruits, I am aware of the trains as situated at the Batignolles train station, as much as I am aware of Pierre Schaeffer's manipulations of them. As such, in this meta-document, there are three geographically and temporally distinct frames: the Batignolles train station, the RTF studio, and my current place of audition. As explored in chapter one, the phonographs of dub music present these three clear distinctions. As I will outline in chapter four, this meta-document type is prevalent among soundscape composers and environmental sound composers more generally.

While at a theoretical level musique concrète may have been considered more successful had it developed a musical framework that embraced its source 
materials, the legacy of Schaeffer's pursuit of absolute music continues to inform electroacoustic composition. As Teruggi, current director of the GRM, writes:

\begin{abstract}
Even if the operational rules disappeared quite early in the history of GRM and of musique concrète, their influence has continued through time as an important reference thus generating a kind of universal rule: do not forget that a sound, before signifying something, is a sound, and has to be mainly considered as that. This idea permits any sound to be considered as a possible sound for music. We should always look for the sound 'itself'!
\end{abstract} (2007, p. 215)

The tenacity of absolute music in electroacoustic music practices is undeniable, as is the continued presence of its longest and most enduring tension: how to deal with mimetic materials. Highlighting this tension is significant, especially when tracing the development of environmental sound composition, a genre that is greatly influenced by electroacoustic composition. In order to better understand this tension, it is useful to trace perspectives regarding mimesis within the electroacoustic music community, examining both historical and contemporary assessments. I will now turn to this topic.

\title{
Mimesis and early electroacoustic composition
}

To reiterate, mimesis, as Emmerson defines it, denotes 'the imitation not only of nature but also of aspects of human culture not usually associated directly with musical material' (1986, p. 17). In addition to recordings of environments or trains, we might include certain types of sounds that imitate birdsong, water or other types of natural or cultural phenomenon. As discussed, in the early years of studio experimentation, there was simply no place for mimetic materials in electronic composition. Musique concrète went to great lengths to disguise the origins of its materials, and though Elektronische Musik used electronic instruments in place of acoustic instruments, imitation of such instruments was not the motivating force. Rather, as Robin Maconie has it, 'electronic music in the postwar era was not intended to sound natural, rather it was considered a medium of sonic purity' (2005, p. 127). In this way, the relationship between 
electronic instruments and acoustic instruments in Elektronische Musik is not mimetic, rather synthesisers were seen as an extension of instrumental capabilities, without the obvious signatures of human agency. Of course, the sound of a synthesiser is mimetically linked to the synthesiser itself, but the distinction made here is that the synthesiser is not transduced in the same way the materials of musique concrète were, and that their sound palette was - at the time - considered thoroughly self referential, as we can infer from Maconie's comment.

The reasons for the aversion to such materials find their roots in the deeper resistance to imitation in western art aesthetics. While I cannot account for this resistance in its entirety, a compelling explanation comes from Douglas Kahn, who summarises a common perspective in post-WWII thinking:

Music was valued as a model for modernist ambitions toward selfcontainment, self-reflexivity, and unmediated communication. Its abstracted character was already thought to have achieved what the other arts were attempting. (1999, p. 105)

In other words, while painting in particular was undergoing a massive transformation from a representational art to a self-referential practice, culminating in the works of abstract expressionism of the post-war period, music through Serialism, was seen as having already undergone such a transformation. To include mimetic materials in electronic compositions would have been considered anti-modern ('antimusical' as Schaeffer had it), a backwards step into the realm of the representational. The programmatic (thus mimetically inclined) tendencies of Romantic period composers were thought to be thoroughly outmoded by the middle of the $20^{\text {th }}$ century. Resistance to mimetic material in electroacoustic composition was pervasive in the 1950s and 1960s, and it wasn't until Luc Ferrari created a thoroughly mimetic work, Presque Rien No 1: Le Lever du jour au bord de la mer (1970) that electroacoustic music would be forced to consider the placement of such materials in its repertoire. As I will detail shortly, 
Ferrari's piece is significant on a number of levels, but I will begin by considering its impact within the genre of musique concrète. ${ }^{34}$

\section{Luc Ferrari and the anecdotal work}

Before exploring Ferrari's work, I would like to clarify an important term used in this section and for the remainder of this thesis. I will use the term 'field recording' to denote what might best be considered untransformed field recordings, though this itself is not an entirely accurate description. An untransformed field recording, in its most precise form, would consist of a single field recording, without splicing, or any post-production, such as equalisation or dynamic control. My use of the term 'field recording' includes untransformed recordings, and also recordings that may have been subjected to splicing and post-production, though such techniques serve to promote the field recording as a pseudo-document. In other words, 'field recordings' is used to denote untransformed recordings and those that simulate untransformed recordings. Presque Rien No. 1 is exemplary of such a simulation.

Ferrari, an instrumental composer, as well as a key member and one-time director of the Groupe de Recherche de Musique Concrète, was well positioned to have Presque Rien No. 1 noticed, given his prominence in both electronic and acoustic composition circles throughout the 1960s. The piece is a subtly edited, $20^{\prime} 43$ " reduction of a recording made in 1968 at Vela Luka, at the time a Yugoslavian (now Croatian) fishing village from daybreak. There are none of the manipulations of materials common to works of musique concrète; it is, ostensibly, heard as a single take recording of an environment. The piece took two years to complete and was released by Deutsche Grammophon on the A-side of a 12-inch record. The B-side contained a recording of a performance of Société II (Et Si Le Piano Était Un Corps De Femme). Placing his field recording alongside an instrumental work, released on a respected classical music label attracted

\footnotetext{
34 Eric Drott provides a comprehensive account of Presque Rien No.1, the political climate surrounding arts practices in France during the 1960s and Ferrari's own desires for environmental sound composition, in his essay 'The Politics of Presque Rien', published in Sound Commitments: Avant-garde Music and the Sixties (2009:145-166)
} 
attention. Presque Rien No. 1 was not a tentative compositional study; it was a fully developed and coherent work by a well-established composer.

Ferrari's piece represents an interesting work in many respects. To begin with, the piece is similar to that of the pseudo-documents of classical music recordings, though the 'work' concept may be theoretically different. Regarding intention, Eric Drott recounts Ferrari's perspective on his own work:

\footnotetext{
For some commentators the minimal intervention in the source recordings that make up Presque Rien represents a tacit repudiation of the work concept central to Western art since the late eighteenth century. Some of Ferrari's comments support such a reading; he has described the work as "a sort of anti-music," through which he expresses his opposition to "the bourgeois myth of the composer." (Ferrari in Drott, 2009, p. 145)
}

It seems that the "myth of the composer" and the concept of the work (be it a nominalist or analytical conception) was prominent among composers of musique concrète in 1970. As Ferrari recounts,

It was badly received by my GRM colleagues, who said it wasn't music! (Laughs) I remember the session where I played it to them in the studio, and their faces turned to stone... I was quite happy, because I thought it wasn't bad at all. (Ferrari in Warburton, 1998)

As the comments by Drott and Ferrari attest, the tension surrounding mimesis in musique concrète was laid bare in this piece. As Drott in particular intimates, the inclusion of mimetic materials in a work presented a challenge to musique concrète on two levels: the status of the composer (and in turn, the composition), and the status of absolute music. Absolute music was thought unattainable where mimetic materials were used, hence Schaeffer's extensive attempts to remove source referents from his materials. Ferrari's piece was vastly more referential than the early studies of Schaeffer made 20 years earlier. Secondly, the lack of overt manipulation and syntactic arrangement of materials ${ }^{35}$

\footnotetext{
35 Simon Emmerson uses the term 'syntax' to denote the arrangement of materials in electronic composition. See Emmerson, S. (1986).
} 
challenged the status of the composer as having special access to the work. With this in mind, we might conclude that behind their 'stony faces' Ferrari's GRM colleagues might have been questioning the validity of the recording as 'music', and the validity of the engineer as 'composer'. These two ideas reside at the heart of the continued tension surrounding field recording and electroacoustic music.

Considering for a moment possible responses to his work and the nature of the phonograph it presents, a listener may perceive the Yugoslavian fishing village as a concrete event albeit temporally, spatially and contextually dislocated from its source. In this way a listener perceives both the event and its subsequent abstraction as implemented by Ferrari and presented by the phonograph. Some may not hear Ferrari in this work, as they attend solely to the materials in the recording. Others may perceive Ferrari's presence through his lack of overt manipulation. Unlike the meta-documents of dub music in which a composer's presence is felt through performance, field recordings offer a more open relationship between a listener and composer: they are much like the pseudodocuments of classical music, though the engineer is more prominent in field recordings, due to the lack of an instrumental 'work' to attend to. We might assume that in searching for the work (if such a search is undertaken), a listener will most often encounter the engineer. As discussed earlier, in works such as Studie II, a confused duality may exist between the concrete phonograph and the abstract work: the work is presented as nominalist in concept, yet the process and sonic signatures of recording marries the work to the phonograph. No such confusion exists here. A listener is most likely to respond (in some way) to the original context of the recording and the new phonographic context created by Ferrari's recording. In fact, the absence of a traditional nominalist conception of the work allows for the nature of Ferrari's intervention to be seen with greater clarity and emphasis than is typically observed for the audio engineers of classical music recordings, though their interventions are essentially of the same order (they both create documents or pseudo-documents). The difference is that Presque Rien No.1 lacks the precise intentional object that the recording of the nominalist instrumental work presents, thus a listener is more inclined to hear 
the framing devices of Ferrari while ignoring the same framing devices utilised by engineers of classical music. The idea of intentional objects, as they pertain to environments and recordings of environments, will be explored in chapters three and four.

Regardless of how his colleagues felt about Ferrari's piece, the arrival of field recordings as the sole material for electroacoustic composition was embraced, especially in North America, where it resonated with the pan-aurality of John Cage and the growing acoustic ecology movement in Canada (see chapter four). Such recordings would not be easily amalgamated into the formal language of electroacoustic music, though there are some notable exceptions. Simon Emmerson and Denis Smalley in particular have closely considered the various intersections of mimetic materials and electroacoustic composition, but for the most part, electroacoustic composition that utilises field recordings as a primary source for composition continue to be compartmentalised into the subgenres of soundscape composition or phonography (a specific genre of field recording). Before discussing such works, I would like to consider the convergence of Elektronische Musik and musique concrète into the broad genre of electroacoustic composition, and the way in which one contemporary theorist in particular has dealt with source-bonded ${ }^{36}$ (mimetic) materials.

\section{Emmerson's appraisal of mimesis in electroacoustic works}

To state that electroacoustic composition continues to reject mimetic materials is disingenuous, though to suggest that the electroacoustic composition fully and easily accepts mimetic materials into its language is equally so. In practice, many composers shift between mimetic and abstract materials in single works, not always concerned for the arguments surrounding their usage. Perhaps the most useful account of mimetic materials within electroacoustic composition is Simon Emmerson's. Emmerson's influential chapter “The Relation of Language to

\footnotetext{
36 The term 'source-bonded' was first used by Denis Smalley to denote sounds as representative of their sources. See Smalley (1997).
} 
Materials" (1986, p. 17-39) provides a map of compositional materials and their arrangement on a 'language grid' (Fig.1):

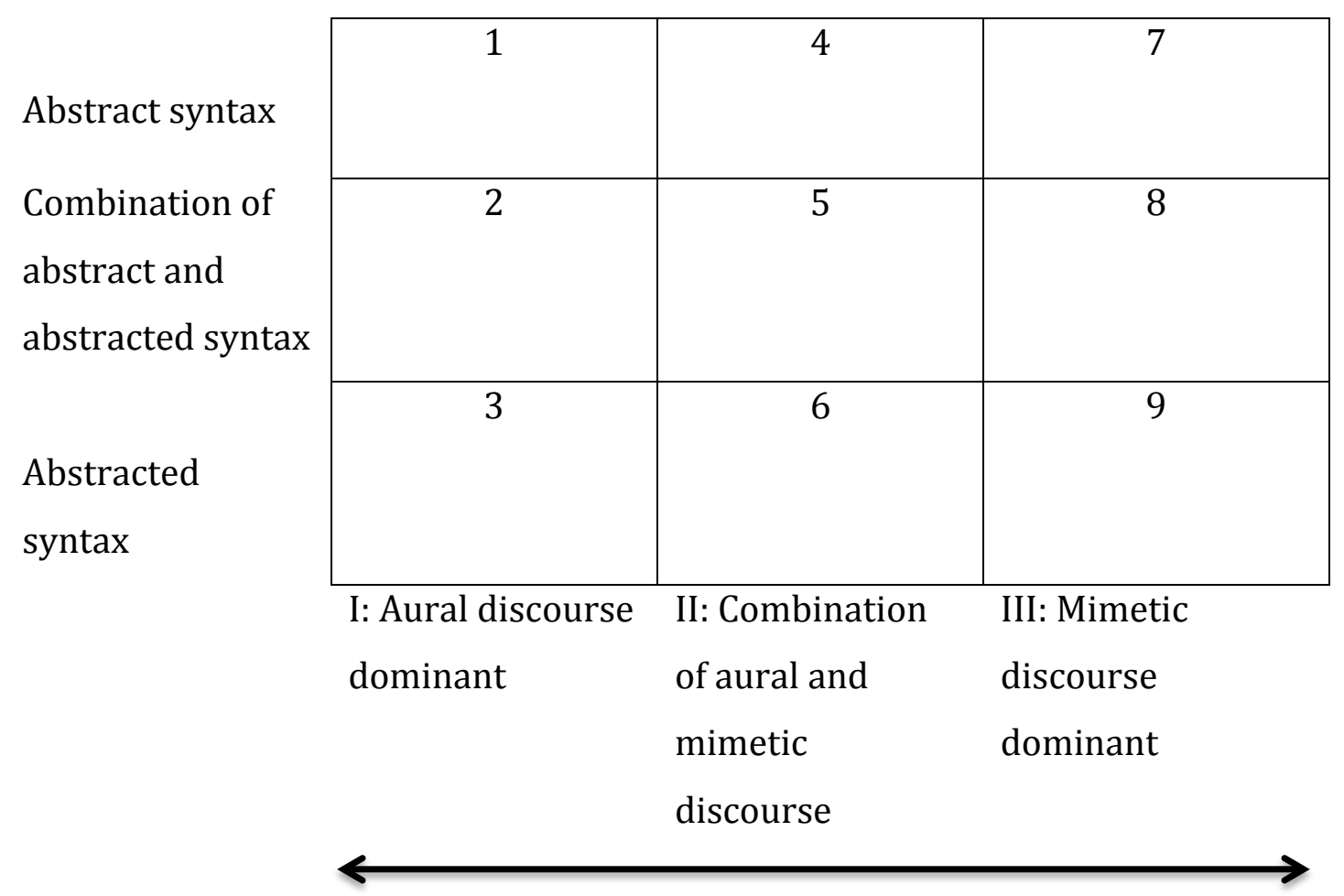

Musical Discourse

Figure 1. Emmerson's Language Grid (Emmerson, 1986)

Broadly speaking, the horizontal axis, musical discourse, refers to the type of materials presented, and the vertical axis considers the syntactic arrangement of these materials. The numbers that appear in the boxes are used to reference his discussion of each of the nine categories later in his analysis. In order to understand Emmerson's grid, it is important to look closely at the terms he uses.

The axis of musical discourse refers to the materials present in a composition. On the left side exists aural discourse, materials that are abstract in nature without recourse to the sonic environment either literally or metaphorically, and on the right, mimetic discourse, whereby materials are either derived directly from sound environments as recordings or are entirely anecdotal of such environments. Appearing at the top of the vertical, syntactic axis we have 
abstract syntax, in which materials are arranged in a manner that derives from an extrinsic (non-musical or sonic) source, such as the twelve-tone techniques of Schoenberg or Cage's application of the I Ching as a governing force in his compositions. Abstracted syntax denotes an arrangement derived from the materials themselves.

For my purposes it is not necessary to recount the 9 intersections of 'syntax' and 'musical discourse', but I will note two extremes: position 1, and position 9. At position 1, aural discourse with abstract syntax, we find Stockhausen's Studie II. As Emmerson notes, 'application of serial principles to electronic sources gives us the clearest case in this first category'. At position 9, mimetic discourse with abstracted syntax, we find Ferrari's Presque Rien No. 1 and other such minimally edited environmental recordings. Emmerson points out that 'there may be reasons for certain choices - location, time of day, duration - which remove the work from the entirely arbitrary' (1986, p. 38). Emmerson is alluding to the persistent perception that field recordings are arbitrarily arranged; in other words, that the syntax of environmental composition is not truly abstracted from the materials but inherent in them, which is in turn linked to the preconception that audio recording is a documentary act, not a creative one. Emmerson notes that the notion of abstracted syntax in environmental works is shifted from the common site of electroacoustic arrangement (the studio) to the site of recording, where techniques such as editing and montaging are replaced with other, situated considerations such as microphone selection, positioning, configuration, and framing devices. Emmerson concludes: 'the will of the composer, far from abdicated, is crucial' (p. 38). In acknowledging this, Emmerson emphasises the role of the composer in the abstraction process, thereby aligning the status of the composer with that of the photographer. ${ }^{37}$ I will focus on the role of the composer in environmental sound composition, as well as the relationship between phonography and photography in later chapters, but for now I will note that though the 'will of the composer' is crucial in the production of works, so too

\footnotetext{
37 Photography was (reluctantly) afforded the status of 'fine art' in the 20th century through the work of Alfred Stieglitz, John Szarkowski and Susan Sontag among others. Szarkowski's The Photographer's Eye (1966), Looking at Photographs (1973) and Sontag's On Photography (1977) are considered highly influential texts; helping to establish photographers as artists.
} 
is the role of the listener, and it is this role that has been marginalised in environmental sound composition and electroacoustic music more generally. The role of the listener will shortly become a central theme of this thesis.

Returning now to the continuum of phonograph types, I noted earlier that though electroacoustic composition is, at face value, the most likely place to find the phonograph-as-work, there are in fact a number of different document types presented. Between Studie II and Presque Rien No. 1 are works that may reside more comfortably in the category of phonograph-as-work. In many respects, the montages of musique concrète are readily understood in this way, especially those that successfully obscure the origins of their source content. Emmerson uses the example of Bernard Parmegiani's De Natura Sonorum (1976) to illustrate grid position 3 (aural discourse with abstracted syntax), which is the most appropriate place to find the phonograph-as-work, of which De Natura Sonorum is a very appropriate example. In my view, Parmegiani's work presents an immediacy of materials and intent that is rare in electroacoustic works. Without mimetic references, a listener is led to hear the materials presently; that is, with attention arrested, and with less opportunity for the kind of nostalgia or reflexivity associated with materials with identifiable sources. Additionally, with a focus on abstracted syntax, where the arrangement of materials is responsive to qualities of the materials themselves, we may hear Parmegiani as engaged not with an abstract and remote concept of the work, but a present and embedded concept that testifies to the work's materiality and phonographic specificity. Parmegiani's presence may even be obscured in this process, as the relationship between the materials and their arrangement may focus attention away from the composer altogether. While it is possible that a work like Studie II may also present a phonograph-specific work to a listener, as indeed may other works with 'aural discourse', it is the potential nominalist reading of such a work that hinders our apprehension of it as purely phonograph-specific; Stockhausen's published score and the work's adherence to (a)tonality and serial arrangement work to support a nominalist reading. In the case of De Natura Sonorum, a nominalist reading seems much less likely, though such a reading is not impossible. Where electroacoustic composition is concerned, whenever a 
listener encounters materials they cannot identify, arranged in a way that feels reflexive to the materials themselves, they will encounter the phonograph-aswork, as their focus is not drawn to the source of the materials, nor to an external source of structure: to a greater extent, the phonograph is the primary provider of context, and thus the primary object of attentional focus. ${ }^{38}$

Having examined the phonograph-as-work in electroacoustic composition, it is apparent just how specific the nature of the work must be in order for the phonograph to be appreciated as the work itself. In most cases, the 'documentary' feature of the phonograph will present itself to a listener in some capacity. The role of the listener in the apprehension of the relationship between materials and arrangement, and document types more generally, is something that should not be understated. In my view, Emmerson's grid is an excellent resource for defining the parameters of electroacoustic music as understood by those composing it. As he himself notes in his conclusion, the grid neatly accounts for the works of musique concrète and Elektronische Musik, while including the more recent development of environmental composition and the possible combinations in between. Yet in reevaluating his language grid, Emmerson notes:

In the intervening years many questions have been raised about the choice of works and the judgment as to where they lie in the grid. There are also larger questions as to where the grid itself stands in the 'poietic/esthesic' distinction and hence its possible roles in analysis and composition. (Emmerson, 2013)

Emmerson's grid goes a long way to formally accounting for the complexities of the electroacoustic 'work'. However, the role of the listener is conspicuously absent. Emmerson alludes to the contention surrounding the grid's 'poietic/esthetic distinction', and it is fair to conclude, as Emmerson does, that while the grid may help provide an account of compositional methods, it does not account for phonographic appreciation: 'We hear works differently over time, so perhaps the work itself can 'walk' across the grid' (2013). Here

\footnotetext{
38 In some instances, listeners who cannot identify the source of a work's materials, or any rational for their arrangement, may look to provide their own narrative. See McCartney, A. (2000).
} 
Emmerson identifies an 'esthetic' understanding of phonographic work I am at pains to convey: though we may usefully consider the phonograph along a continuum, within a grid, or with any formal tools, the listener alone holds the key to interpretation, an interpretation that may change over time, and one that may not conform to poietic intent.

Before moving on to discuss intentionality, a core theme in chapter three, it is important to reiterate the value of exploring audio recordings as types of phonographs. Though certain genres of music may exhibit unique characteristics in the way they relate to audio recordings, designating phonograph types helps to reveal commonalities between seemingly disparate genres, even in cases where genres appear to have totally opposing values, such is the case with classical music and punk music (see chapter one). As discussed in the present chapter, many electroacoustic works have a great deal in common with other phonograph types. In instances where materials are not stripped of their source referent, electroacoustic works exhibit characteristics of the meta-document: the listener hears the source materials with the composer performing as a spatially and temporally dislocated arranger, intervening between the materials and the listener. As I will argue in chapter four, the majority of soundscape compositions exhibit such properties. In this way, many electroacoustic compositions find comparisons with recording types within the world of popular music. In my view, there is a great deal of commonality between Schaeffer's Etude Aux Chemins De Fer, and Hopeton Brown's dub mix of Johnny Osbourne’s “Over 31, Under 21" (1982): they both reevaluate mimetic materials through technological manipulation, searching for new musical forms derived from the materials themselves. Though electroacoustic composers may be offended by the suggestion their works bear similarity to the music of Hopeton Brown (or any form of 'popular' music), there is value in such a comparison. If we consider recordings based not on their genre but on their treatment of documentary and abstraction, the position of the work, the temporal dimensions of production and the interactions of materials and arrangement, then we may begin to see greater cross pollination and understanding between genres, and greater consideration of the phonograph itself as a primary focus of listener engagement: for though a 
composer may wish that a listener hears a nominalist work, a performance, or a soundscape, in truth a listener hears a phonograph. 


\section{3}

\section{Intentionality, Environmental Listening and Field Recording}

The relationship between the phonograph, environmental sound, listening and composition is a complex and widely misunderstood one. As I will outline in the first part of this chapter, listening to environmental sound as a situated experience engages with notions of intentionality, including environmental 'musicalisation' as a form of 'aestheticised listening' (Hollerweger, 2011, p. 18). In the second part of this chapter, I will detail how listening to mediatised environmental sound is equally complex. Notions surrounding phonographic impartiality, composer expectations, the rendering of a multi-modal environment to a single sense (i.e. mono-modality), and the ability of those attending to the phonograph to engage with situated experience are brought to the fore. Abstraction and documentary are also discussed within the context of field recording in order to understand the way in which the phonograph is thought to operate within the genre, a genre that will be further discussed in chapter four. In all, while environmental sound composition may sit neatly within Emmerson's grid of musical discourse for utilising mimetic materials with abstracted syntax (as discussed in the previous chapter), there exist a variety of complex issues that warrant an extensive investigation into the genre, as Emmerson's simple description, useful and important as it is, does not account for this complexity. This chapter explores the notions of intentionality, environmental listening and the role of the phonograph in field recording as a platform from which to discuss acoustic ecology, soundscape composition and listener reception in chapter four. Chapters five and six also extend ideas 
presented in this chapter in the context of sensorial perception and hermeneutic analysis respectively.

I will also begin to reference my own compositional practice in this chapter, and will continue to do so in chapter four. I do so because the majority of my works are directly influenced by the core theoretical concerns that appear at the intersections of composer intentionality, phonographic intervention, and listener experience of environmental works, discussions of which begin here in chapter three. My compositions directly illustrate how I explore these relationships within the context of my artistic practice. Not all of the works presented in Volume II will be detailed in this chapter and the next, just those that directly relate to the specific themes addressed. As most of my pieces address multiple themes and ideas, I will detail their production methodology and aesthetic concerns in the second volume alone.

\section{Intentionality}

With little doubt, a composer's fascination with lived experience of the sonic environment resides at the heart of environmental sound composition. This is perhaps best evidenced within the genre of soundscape composition, in which composers attempt to promote their engagement with the environment through composition. This is an idea I will rigorously assess in chapter four. But before we can discuss compositional motivations where recorded environments are concerned, it is important to address the underlying relationship between humans and the natural world, specifically through the ideas of perception and intentionality; two notions that are crucial to any discussion of environmental sound composition, as an understanding of the meeting place between environment and human perception reveals a great deal about composer intent and listener response, while also shedding light on the nature of the phonograph within the genre.

While a detailed account of Edmund Husserl's concept of phenomenological intentionality is well beyond the scope of this thesis, I would like to provide a 
brief overview of his ideas, as they help provide a framework for understanding environmental sound composition and electroacoustic music more generally, on a number of different levels. To begin, and from a broader perspective, Intentionality is defined as 'the power of minds to be about, to represent or to stand for, things, properties or states of affairs', ${ }^{99}$ or as Husserl has it, intentionality is 'the fundamental property of consciousness' (Husserl in McIntyre and Smith, 1989, p. 147). Using a method termed 'phenomenological reduction', Husserl posits the world as constituted in consciousness. That is not to say that the world exists in our minds, but that our relationship to the world is 'achieved in the act of consciousness' (Russell, 2006, p. 86). Husserl's reduction ('epoché') is a methodology designed to promote a phenomenological understanding of perception. As McIntyre and Smith have it, 'its purpose is to force us to explain the phenomenological features of acts, including their intentional character, by appealing only what is intrinsic to the acts themselves: to the internal structures of acts that make them the mental states or experiences that they are' (1989, p. 152).

As suggested in chapter two, we can clearly observe the influence of Husserl's reduction in Schaeffer's ecouté reduit. Brian Kane notes of Schaeffer's Traité des objets musicaux (1966) that:

\footnotetext{
Throughout the Traité, Schaeffer remains quite close to the letter of Husserlian phenomenological orthodoxy, often calling upon it when trying to articulate his views on the sound object, reduced listening, and the acousmatic field. Consistently, Schaeffer deploys techniques that are Husserlian in character: the transcendental-phenomenological reduction, the eidetic reduction, imaginative free variation, and the reactivation of originary experience. (2014, p. 19)
}

As this shows, Schaeffer promoted a method of cognitive reduction or bracketing in order to attend to the intrinsic properties of his sound objects. As I will explore in chapter four, this methodology still carries influence within the world

\footnotetext{
${ }^{39}$ Jacob, Pierre, "Intentionality", The Stanford Encyclopedia of Philosophy (Fall 2010 Edition), Edward N.

Zalta (ed.), URL $=<$ http://plato.stanford.edu/archives/fall2010/entries/intentionality/>. Retrieved 22 August 2014
} 
of environmental sound composition. Indeed cognitive bracketing, albeit with varying emphasis, is a technique used in many forms of listening, both situated and mediatised, and I will continue this thread throughout this chapter. For now I would like to consider what Husserl believed his phenomenological epoché allowed an individual to discover: their unique phenomenological intentionality.

Husserl considers his intentionality in three parts, the 'hyletic data', which is understood as the sensory information received by the subject, allowing limited access to the object (an 'adumbration' or partial object); 'noesis' or the intentional act of perception; and the 'noema' or the intentional object of perception. Let us consider an example of how an individual may perceive in these terms. A lone Tui (a native New Zealand bird) sings in a tree, out of sight. The (aural) hyletic data imparts a sense of the Tui: we experience an adumbration of it through the limited data we receive. Having encountered an adumbration of the Tui, we intentionally perceive the Tui - it is constituted in the act of noesis - and the Tui is thus objectified and endowed with our intentional perspective; it is now a noema (intentional object). Importantly, in Husserl's intentionality, a Tui remembered, or a mythical Tui is no different from a proximate or 'real' Tui, as they are all objects of perception. As such, while Husserl's noema may seem to point to a distinction between the natural world and the perceiving mind, the two are in fact integrated in the act of perception; such is the core principle of phenomenology. Though there has been more recent work in the area of phenomenology that has sought to extend Husserl's ideas, ${ }^{40}$ it is the core principles of intentionality that I wish to address here. Intentionality then,

Is something we know about first and foremost from our own, "first person" knowledge of our experiences and their "internal" character; that is, a property our experiences have in themselves, as subjective experiences, and independent of any of their actual relations to the external world; and that therefore intentionality cannot be explained from a purely objective, "thirdperson", point of view if such a viewpoint cannot accommodate this internal

\footnotetext{
40 Graeme Harman's book Guerrilla Metaphysics: Phenomenology and the Carpentry of Things (2005) is
} exemplary of a renewed interest in Husserl's ideas, including the ideas discussed here. 
and subjective character of our experiences.' (McIntyre and Smith, 1989, p.

151-152)

Importantly, a noema may retain an air of objectivity (albeit a subjective objectivity) while our 'noetic' analysis of it may change radically. For example, a person may have once found the sound of church bells (the intentional object) to evoke feelings of community and reverence, may now, having lost religious conviction, find the same bells to evoke entirely different feelings. An individual's perception may change radically, though the intentional object may stay the same. This understanding of perception has far-reaching consequences for environmental sound composition, but for the moment, I make this observation: though intentional objects (the Tui, for example) may appear to hold universally perceptible qualities due to their existence in the natural world, in fact such objects are constituted in noesis, and are therefore deeply related to our prior experiences of such objects. In no way then can one individual's intentional object be exactly identical to another's intentional object. While this may be the case, a degree of commonality may exist between the noetic responses of individuals; that is, a form of inter-subjectivity derived from the shared experience of hyletic data. Such inter-subjectivity may indeed occur to some extent, but in my view it is the assumption that inter-subjectivity will occur that promotes a false objectivity in the perception of a noema; that is, a supposed merging of an individual's intentionality with another's. As I will demonstrate in this chapter and the next, this assumption is prevalent in many forms of environmental sound composition.

It should be noted that a reading of intentionality in which noesis is considered tied to the individual that perceives (i.e. without a focus on inter-subjectivity), provides a particularly useful tool when analysing environmental sound and its relationship to the phonograph, as it promotes a fundamental understanding of perception that I hold to be widely experienced; one in which listeners perceive not only environments differently, but their recording also. In my view, intentionality governs our perception of all events, be they situated or mediatised, and accounting for common traits of intentionality in these 
situations is crucial to the discussion of environmental sound composition, for when we unpack the intentionality of composers and listeners alike, we begin to understand the perceived role of the phonograph more clearly.

In the following section, I will discuss the nascent and widespread desire to incorporate environmental sound into music in the first half of the $20^{\text {th }}$ century, with a focus on the situated listening practices of John Cage with further observations from John Andrew Fisher. The purpose of this section is to reveal the role of intentional cognitive bracketing in the 'musicalisation' of the environment, highlighting the way in which this bracketing functions and the difficulties it presents to an inter-subjective appraisal of the environment. I will also discuss the nature of technological framing, and the relationship between such a frame and those who used it to record environments. Of significant interest to this initial discussion of environmental musicalisation, are the theories of John Cage, one of the most influential figures in late $20^{\text {th }}$ century western art music and environmental sound composition in particular. Though the theories of Cage provide a useful place to commence a discussion of the incorporation of environmental sound into western art music, I have elected to begin with earlier works and composers. This serves to contextualise Cageian theory within a broader movement of environmental sound in art.

\section{Intentionality and the environment before Cage}

Although John Cage and his notions of 'all sound', 'always sound' and 'panaurality' (Kahn, 1999, p. 159) are considered highly influential upon many environmental sound composers, his attention to everyday 'non-musical' sounds and his desire to incorporate them into more 'musical' contexts was by no means a new idea. Famously, Luigi Russolo had expressed a very similar desire in his Futurist Manifesto The Art of Noises (1913). In his manifesto, Russolo calls for a new music, in which environmental sounds feature strongly. He also calls for a new kind of situated listening. Writing to prominent Italian Futurist Francesco Balilla Pratella, Russolo implored: 
Let's walk together through a great modern capital, with the ear more attentive than the eye, and we will vary the pleasures of our sensibilities by distinguishing among the gurglings of water, air and gas inside metallic pipes, the rumblings and rattlings of engines breathing with obvious animal spirits, the rising and falling of pistons, the stridency of mechanical saws, the loud jumping of trolleys on their rails, the snapping of whips, the whipping of flags. (Russolo, 1913)

Russolo's notion of the 'attentive ear' suggests a kind of aestheticised listening to everyday sound that is, as I will discuss, Cageian in character. It also contains aspects of Husserlian bracketing. Russolo's suggestion that his reader walk through a city 'with the ear more attentive than the eye' suggests a cognitive diminishing of visual perception in order to promote aural perception; a kind of sensorial reduction aimed at focusing on sound aesthetically. Though Russolo would not marry his desires for everyday sound in music with the phonograph (Russolo instead invented intonarumori; hand-cranked, noise-making devices used to imitate the sounds of industry and nature), other artists would. In Noise Water Meat (1999), Douglas Kahn recounts the first experiments of Russian filmmaker Dziga Vertov, performed in 1916, with audio technology:

\footnotetext{
I had the original idea of the need to enlarge our ability to organize sound, to listen not only to singing or violins, the usual repertoire of gramophone disks, but to transcend the limits of ordinary music. I decided that the concept of sound included all of the audible world. As part of my experiments, I set out to record a sawmill. (Vertov in Kahn, 1999, p. 140)
}

Vertov's suggestion that 'all of the audible world' is suitable for music, is very similar to Russolo's (and later, Cage's), though where Russolo's desire to utilise environmental sound was realised through mechanical mimicry, Vertov realised the same desires (or at least planned to) through recording technology; it has been suggested that the poor quality of recording fidelity in 1916 saw Vertov abandon his concept of a new music, instead focusing his attention on cinema (1999, p. 140). Russian avant-garde film featured heavily montaged and often asynchronous audio throughout the 1920s and into the 1930s, and though these experiments seemed to have little impact on the world of western art music, they 
did influence other non-Russian filmmakers, including Walter Ruttmann, a German filmmaker who transferred the montage technique of film to the medium of radio. His piece Wochenende (1930), a piece alluded to in the previous chapter, is an 11-minute audio montage of recordings made around Berlin. Created as a kind of city portrait, Ruttmann clearly develops the ideas of an acousmatic art that Vertov considered some 15 years earlier. Upon releasing Wochenende, Ruttmann wrote:

Everything audible in the whole world becomes material. This infinite material can now be given new meaning by fashioning it in accordance with the laws of time and space. This opens the way for a completely new acoustic art - new in its means and in its effect. ${ }^{41}$

Like Vertov before him, Ruttmann promoted the notion of 'everything audible' as suitable for art, and in many respects, he realised in 1930 the very art that Vertov had called for in 1916. With his 'completely new acoustic art', Ruttmann successfully married environmental sound with the phonograph, albeit within the context of radio, paving the way for musique concrète, though it appears Wochenende had little or no influence on Schaeffer's practices (given the wealth of material authored by Schaffer with no mention of Ruttmann's work). The works and ideas of Russolo, Vertov and Ruttmann anticipate Cage's call to bracket environmental sound both in situ and through artistic intervention. In Husserlian terms, they propose intentionality toward the environment that brackets it as a noema suitable for aesthetic evaluation, and ultimately suitable for incorporation in the arts generally. Following in the footsteps of these pioneers (Russolo in particular), John Cage attempted to incorporate environmental sound into music specifically.

41 (Ruttmann, W. 1930: http://www.medienkunstnetz.de/works/weekend-remix/, accessed 8 April 2009) 


\section{Cage}

In The Future of Music - Credo (Cage, 1937/2004), John Cage, much like Russolo, Vertov and Ruttmann before him evaluates the potential for everyday sounds for use in a musical context:

Wherever we are, what we hear is mostly noise. When we ignore it, it disturbs us. When we listen to it, we find it fascinating. The sound of a truck at 50 M.P.H. Static between the stations. Rain. We want to capture and control these sounds, to use them, not as sound effects, but as musical instruments. (Cage, 1937/2004, p. 25-26)

A type of Husserlian bracketing is implied in Cage's statement. He notes that in order to hear environmental sound as 'fascinating' (not 'noise') we must 'listen'; that is, intentionally bracket environmental sound as a noema of aesthetic value. Cage also expresses the desire to 'capture and control' sounds of the everyday, which must surely reflect the necessity to record them; an idea that had been realised by Ruttmann seven years earlier and would be developed by musique concrète some ten years later. Interestingly, Cage's early works utilising recording technology were, in theory, similar to those of Russolo. Gramophones replaced traditional instruments in an ensemble setting in much the same way Russolo's intonarumori sought to replace orchestral instruments. Cage's Imaginary Landscape No.1 (1939) is the first of his works to utilise recording technology in an ensemble. Earlier works for gramophone by Toch and Hindemith directly inspired his composition: as recounted in chapter one, the 17year-old Cage was present for the premiere of Originalwerke für Schallplatten in Berlin, 1930 (Katz, 2010, p. 123).42

A musicalisation of the non-musical, that is, an intentional, noetic perspective that frames sound as musical, would inform many of Cage's works throughout the 1940s and beyond, not least of all his most (in)famous work, 4'33" (1952). 4'33" is a three-movement piece for unspecified instrumentation in which no notes are

\footnotetext{
42 Though Cage would continue to incorporate audio technologies including radios and tape machines into ensemble performances in many of his pieces, it wasn't until the1950s that he wrote his first piece entirely for the phonograph, Williams Mix (1953).
} 
played. Though the work is commonly known as Cage's silent piece, it is the innate lack of silence that is the focus of Cage's piece. The first performance featured David Tudor at the piano at a concert in Woodstock, August 29, 1952. The performance is generally considered a watershed moment for western art music for a number of reasons, the most important of which is that it emphatically broadened the genre in its materiality, allowing for everyday sounds to be framed as musical, that is, listened to as music, paving the way for a number of experimental music practices into the 1960s and beyond.

\section{Cageian intentionality}

The appearance of Husserl's phenomenological intentionality is implicit in Cage's pan-aural musical revolution, and the phenomenological bracketing undergone by his audience (at least some of them) during the Woodstock performance of 4'33" is probably one of the most punctuated arrivals of Husserl's phenomenology into environmental listening in a musical context. In many respects, prior to this performance, musical listening could be thought to reside in Husserl's pre-phenomenological attitude, the 'natural attitude' of perception. Matheson Russell has this to say of the 'natural attitude: 'In it we are oriented towards the world of actual existing things - the real world - and our attention is given over to it' (Russell, 2006, p. 59). Even the more radical works of early $20^{\text {th }}$ century orchestral music - those pieces that questioned the materiality of music could be thought to reside within this perceptual attitude. By way of example, though Stravinsky's Rite of Spring (1913) caused many at the time to question whether the work was in fact music, their assessment of it was made from an already musical ear. In other words, despite protestations, the intentional object (Rite of Spring) had already been designated as a musical object in the perceiving minds of the work's detractors; no phenomenological reduction was required in order to perceive music, despite the questions surrounding its suitability for such perception. Cage's great leap was to expand the preexisting notion of the musical object to include all sounds, all the time. In this way, 4'33" was not so much a musical revolution as it was a perceptual revolution. However, to conclude that Cage managed to pull environmental sound onto the same perceptual playing 
field as orchestral music (or any other performance-based music) is perhaps a stretch; while a great deal of music is typically perceived within Husserl's natural attitude, environmental sound must undergo phenomenological reduction to be heard as musical, just as Cage himself had intimated in The Future of Music Credo (1937/2004).

In Noise Water Meat (1999) Douglas Kahn is critical of Cage on a number of levels, but I would like to focus on one point in particular, as it helps to further refine Cageian intentionality. To begin with, Kahn summarises Cage's perceptual revolution as follows:

\begin{abstract}
Russolo initiated the strategy whereby extra-musical sounds and worldliness were incorporated rhetorically or in fact into music to reinvigorate it. Cage exhausted this strategy by extending the process of incorporation to a point to every audible sounds, potentially audible and mythically audible sounds, where consequently there existed no more sounds to incorporate into music, and he formalized the performance of music to where it could be dependent on listening alone. (1999, p. 164)
\end{abstract}

And later:

After a certain point communication, ideas and intention were also to be expunged so all that was left was a sound in itself. This tendency in Cage was a measure of the degree to which he was lodged within Western art music and how willing he was to carry further its processes of exclusion and reduction with respect to sound in general. It was as though he could legitimately extend the bounds of musical materiality only by proving an unflinching fidelity to musical areferentiality on its own turf. (p. 164-165)

Kahn's criticism of Cage is that his perceptual revolution was only made possible through the uneasy framing of environmental sound with a strictly formalist perception. Regardless of Cage's rational for this specific intentionality, there can be little doubt that absolute music influenced his situated listening practices. As Kahn has it, Cage dismissed the notion that 'sound' held an ability to communicate ideas: "If I am going to listen to a speech, then I would like to hear 
some words" (Cage in Kahn, 1999, p. 164). He goes further in an interview from 1992, explaining that instrumental music seems discursive, while environmental sound is without meaning:

\footnotetext{
When I hear traffic, the sound of traffic here on Sixth Avenue for instance, I don't have the feeling that anyone is talking, I have the feeling that a sound is acting, and I love the activity of sound. What it does, is it gets louder and quieter, and it gets higher and lower. And it gets longer and shorter. I'm completely satisfied with that. I don't need sound to talk to me. ${ }^{43}$
}

Many composers may share Cage's perspective on environmental sound, though as I will discuss in chapter four, many soundscape composers in particular believe that the environment has the ability to "talk", or at least their compositional methodology implies such a viewpoint. Regarding Cage, what is important to note is that he describes Sixth Avenue in musical terms: amplitude, pitch and duration. There can be little doubt that his intentional bracket is deeply influenced by his experience as a composer of instrumental music. His intentional bracketing of environmental sound involves listening for 'musical' qualities alone. In short, Cage ignores the mimetic aspect of environmental sound in order to perceive it as self-referential. In this instance, the bracketing approaches a kind of Husserlian reduction. As Kahn suggests, this positions Cage's intentionality within the long-established ideology of absolute music.

\section{Environmental listening: subjectivity and attentional focus}

John Fisher, in "What the Hills are Alive With: in defense of the sounds of nature" (1998), having established that the sonic environment is worthy of aesthetic appreciation, then argues that the tools used to evaluate music (such as those employed by Cage) are inappropriate in the evaluation of the sonic environment, as for the most part there is no way to gain a consensus on what is to be evaluated and how. Fisher's opposition to evaluating environmental sound with musical ears centers on notion of framing: 'because nature does not provide an intentional object of appreciation the way musicians do, there is a serious

43 Transcribed from the video documentary Ecoute (Sebestik,1992) 
framing problem concerning the sounds of nature: which sounds do I pay attention to and for how long?' (1998, p. 172). That is not to say that individuals do not employ such an assessment, but rather that analysis of the environment cannot be made in these terms. As such, though Cage may have listened to Sixth Avenue from his window, hearing a composition unfold below, and though he may have believed that, through formalism (a reduction of the environment to its spectromorphological qualities), others could attend to the same composition, Fisher believes that such inter-subjectivity is impossible where the environment is concerned due to variances in individual's attentional focus:

\footnotetext{
Suppose you are sitting in a hot tub in a city in the Arizona desert listening to the sounds around you. Do you just listen to the Western Warblers and the wind in the fruit and palm trees or do you (should you) also notice the sounds of the hot tub jets and the popping bubbles making a pleasant hissing on the water? Do you add or ignore the sounds of ventilator fans spinning hot air from attics and occasional jet planes overhead? (1998, p. 173)
}

Fisher's example highlights an important point: no two people will hear a sonic environment in exactly the same way due to their individual predilections for some sounds over others. The occupants will intentionalise the environment with different emphasis. Crudely put, a bird watcher may focus their attention on the Western Warblers, a plumber may focus on the hot tub jets while a composer (such as Cage) may focus on the spectromorphological characteristics of all sounds in audition or their spatial distribution. Additionally, no two people will encounter the same adumbration of the environment. Environmental sound, as it quickly emerges and passes, presents different adumbrations at different times and locations, and as such the very data used to determine the environment is necessarily different between individuals before noesis even begins.

This is where the notion of inter-subjectivity in listener perception falters. Without an orientating device, such as an explicit call to listen in a certain way, (Schaeffer's reduced listening provides a familiar example), inter-subjectivity seems an unlikely prospect, especially where environmental sound is 
concerned. In addition, reaching a consensus regarding when to listen aesthetically is problematic if not impossible, as unlike music, such environments do not have a start and end, nor a clear focal object. This is not to say that individuals do not bracket the sonic environment in such a way:

Consider an example: I am sitting on a rural hillside and the wind is blowing very hard and noisily through the bushes and trees. Suddenly the wind stops and there is a surprising and powerful silence. Then one frog begins to sound, followed by one bird. This interlude lasts for thirty seconds at the most. Then other birds and crickets join in until there is a crescendo of sound to which the wind finally adds an overwhelming whooshing and bustling as it picks up again and drowns out the other sounds. This interlude strikes me as a lovely sonic moment, and my framing of it was no doubt natural. But it was entirely dependent on (Western) musical analogies. Even though it was "natural," this is not the same as universal, nor is it the same as a frame that is dictated by the intrinsic nature of the sound events themselves. (1998, p. 173)

In Fisher's estimation, the cognitive bracket employed when listening to the environment aesthetically is not universal, nor does the environment itself suggest the correct way to listen as instrumental music does. However, composers in particular exhibit certain tendencies when listening to the environment, especially those who seek to record it.

\section{Composers: listening in situ}

As discussed, composers may listen to environments with an intentional bracket that employs musical analogies such is the case with Cage and Fisher in the cited examples. We might conclude that composers such as Pierre Schaeffer and Spanish sound artist Francisco Lopez, a self-confessed 'Schaefferian' (1997), share a similar perception. Where the composers differ, is that Cage listens in this way in order to 'musicalise' environmental sound in situ. I believe that both Lopez and Schaeffer listen with a formalist ear in situ primarily as a function of their assessment of a sound's suitability for phonographic capture and replay as a sonic object. To illustrate the point, I suggest that had Cage and Lopez sat 
together in Fisher's Arizona hot tub, Cage might listen with a formalist ear to intentionalise the environment as music right there and then, while Lopez might listen with a formalist ear to ascertain whether he should go and get his audio recorder, so that someone else might hear the environment (albeit recorded) as music at a later time. Here an important notion arises: environmental sound, in order to be considered suitable for phonographic capture and composition must first be cognitively musicalised by the composer; that is, the environment must first be regarded as an intentional object with aesthetic value. ${ }^{44}$ Or, put in phenomenological terms, in determining the noema of the situated environment, a listener imagines a second noema (a recording of the environment) in their noetic perception. As such, while the phonograph is often considered the first step towards the musicalisation of environmental sound insofar as it gives environmental sound musical parameters such as a fixed duration and repeatability (an idea I will discuss shortly), has in fact already occurred. In this way, environmental sound composition encounters a double-musicalisation that seldom exists for other recorded works: the first is that of the composer, the second is that of the phonograph.

The reason for this double framing relates to our general understanding of authorship and performance. Regardless of whether we adhere to a nominalist conception of the work, performances of compositions are already considered musical, which amounts to an appreciation of their production, including the role of the composer and the performer. These contextual factors help position the performance within the natural attitude of perception; we attend to such performances, be they classical compositions or rock bands, with an already musical ear: no phenomenological reduction is required in order to perceive such performances as musical. As discussed in chapter one, we attend to recordings of these performances in much the same way. However, as just discussed, environmental sound composition requires more effort; specifically, a phenomenological reduction, or at least something akin to it. This is due in part to a conspicuous lack of human authorship of the natural environment. As I will

\footnotetext{
${ }^{44}$ I use the term 'musicalise' here to denote the 'musical' parameters the phonograph offers environmental sound; specifically a temporal frame. That is not to say that environmental sound composers perceive of their recorded works as 'music'.
} 
explore shortly, the phonograph then provides further musical parameters, understood as the second musicalisation. What becomes apparent upon listening to such recordings is that while a listener may easily engage with the musicalising properties of the phonograph, the ability for a listener to engage with a composer's initial and intentional musicalisation is far less certain. At this point I would like to introduce one of my own works that deals specifically with the 'musicalising' properties of the phonograph.

\section{CHPHNO-004 (2015)}

This piece comprises three single-take recordings made in the city of Seoul at night. Like Fisher, as quoted in the previous example, I am sat on a hillside, marveling at the environment around me. My particular intentional frame is operating: I am listening to the environment in situ, and have decided that this particular time and place would make for a good recording. I sit there for some time, cognitively projecting an imagined recording from the current environment. This is the composer's first 'musicalisation' of the environment. I then take out my recorder, and begin the process of creating a second frame with recording technology. I change position a few times between takes to alter the listener perspective. In the process, I am providing additional 'musical' parameters to a situated experience - I have given it temporal and spatial frames.

Back in my studio refine these frames, employing lengthy fades to introduce and conclude the three recordings I have elected to use. The fades are used as a device to promote the environments as existing before and after the frames that 'musicalise' them; abrupt beginnings and conclusions do not have the same effect. I also elected to use long fades in order to soften the onsets and conclusions of the three recordings for listeners; allowing the experience of the work to feel less dictatorial; as the audio emerges from and recedes to silence, so too might a listener's noetic turn in the appreciation of the work emerge and recede. In my view, the way in which the second, 'musical' frame of the work is presented has enormous implications for the listener's response to environmental sound works, an idea I explore specifically in this work, and also in a number of other works. 
The double framing of environmental sound composition is unique, with farreaching influence on the production and reception of its works. Before discussing these dynamics in detail, I would like to briefly return to Schaeffer's intentionality, as regardless of how Cageian intentionality manifests itself in environmental sound composers, Schaefferian intentionality where situated listening is concerned, holds much more universal appeal. In my view, though Schaeffer had a very particular interest and methodology where environmental sound and composition are concerned, his approach to the environment, by holding a phonographic lens to it, is an experience held by many:

For there to be music, all that is needed is that a relationship be established between subject and object, and the initial act in music is willed hearing, i.e., selecting from the chaotic hubbub of sounds a sound fragment that one has decided to consider. Here the memory acts as a closed groove: it retains, it records, it repeats. This fragment must be considered for all it contains: matter and form. It can be repeated imitated, combined with others. Hence a subject could create a music for himself out of a continuous chaotic chain of sound by imposing externally a form that comes from within him. (Schaeffer, 2012, p. 66)

Here we can see that Schaeffer's intentionality is greatly influenced by his experiences with the phonograph. His situated listening experiences are informed by their phonographic potentiality, and as such, we see Husserl's phenomenological intentionality in full effect: much like Cage, Schaeffer approaches the world with a musical ear, though for Schaeffer, his noetic ear is clearly informed by his experimentations with the phonograph. As recounted in chapter two, Schaeffer would concede in later life that he never managed to successfully musicalize environmental sound through the phonograph, and as such, we might conclude that his situated musicalisation too fell short of his ambitions. However, though he may have failed to reach his own objective this is not to say we are unable to hear the environment in such a way. Through his example, many listeners, myself included, have been able to conceive of the environment as recorded, an intentionality that requires a listener to imagine a 
future noema (the phonograph), and project it back through their noetic process to the environment in situ. Moreover, there is often a mismatch between the phonographic lens that the situated composer imagines, and the actual phonographic lens of the microphone and recording apparatus. As such the intentionality of the composer and their relationship with the recorded environment, both real and imagined, plays a significant and complex role in the production of environmental sound composition. It is to this interplay that I will now turn.

\section{Listening through the phonograph}

To begin with, I would like to consider straight (untransformed) field recordings in isolation from those that have been transformed in some way (through editing, layering, montaging, filtering, etc.). The reason for this is to examine the role of the recording in relative isolation from the studio-based manipulations of the composer, which are often applied to establish a new set of aesthetic concerns (I will examine transformed recordings throughout chapter four). In this section and the following, the term field recording is understood in the terms described in chapter two: mediatised lived experience, creating the document or pseudo-document varieties of phonograph. Secondly, I will continue to refer to field recording engineers, phonographers and those who manipulate their recordings in post-production with one term - composers regardless of whether the individuals or genres discussed use the term to describe themselves. I do so as a way to simplify the terminology in this section, and also as a way to acknowledge the fundamentally authorial role such individuals occupy in the creation of their recordings. Finally, while I will at times reveal individual motivations for recording environmental sound, this section is not primarily concerned with the why of field recording, rather the what: by revealing the differences between situated and non-situated listening, I wish to explore the specific role of the phonograph in environmental sound composition. This in turn helps to reveal the complexity of composer intentionality with regard to their works, a discussion of which will begin later in this chapter and proceed with greater focus in chapter four. 
The recording of a sonic environment has many far-reaching aesthetic implications. For example, field recording is often seen to provide environmental sound with the musical parameters Fisher understands as being absent in situ. I described this process earlier as the second bracket or musicalisation of environmental sound composition. At the most fundamental level, a recording has a start and end. As Emmerson would have it, such a framing device constitutes a form of abstracted composition. The natural arrangement that Fisher describes of his rural hillside listening experience can now be embodied in a recording. Additionally, the repeatability of the phonograph allows many to experience this arrangement multiple times, and as such, we can begin to discuss its form. It may even be said that in conjunction with a field recording's framing and repeatability, that attention to certain aspects of the recorded environment may be emphasised above others. For example, a listener may be led to focus on the jets and bubbles of Fisher's Arizona hot tub if a recording begins with the jets being switched on. Temporal framing then, is one aspect of situated intentionality that can be made explicit in the recording process, and while in my view the recording of an environment cannot provide access to a composer's complex intentionality, recording can impart core aspects of it. However this is by no means a simple transaction: while temporal framing and repeatability serve to musicalise a sonic environment, the issue of what to focus on remains. In other words, the act of recording an environment does not necessarily musicalise its content as a composer may intend. In fact, in some situations the intent of the composer is thoroughly undone by the recording process itself. This points to a very specific feature of the phonograph where environmental sound is concerned: once microphones are set in place, recordings of environments are impartial; despite what many composers may believe, recordings cannot enact the cognitive framing that a composer experiences in situ. In other words, recording technology has its own kind of intentionality born of its apparatus: its strengths and limitations providing a technological perspective, albeit a non-conscious 
perspective. ${ }^{45}$ Though a composer, as an operator of technology, may select a specific phonographic lens through their choice of microphone, placement and recorder type, the technology itself imparts its own intentionality despite such selections, born of its neutral perspective regarding the materials it records. In order to assure us that this is indeed the case, I would like to explore two examples.

In his book Haunted Weather: music, silence and memory (2004), David Toop recounts a field recording excursion in which his situated listening experience was not captured by his recording:

An Australian composer who combines digital electronics with soundscape recordings - Lawrence English - took me for a walk through rainforest close to his home city of Brisbane. At one point we stopped just to listen. I switched my minidisk to record, and after some time, I was rewarded by the fabulous sound of catbirds - birds whose calls bring to mind images of babies being strangled or something equally as horrible. Back in London I listened to the disc and was surprised by the loudness of motorbikes passing on the road nearby. The scene had been so tranquil that I focused on the sounds that were unfamiliar. The bikes I could hear anywhere, so I subconsciously reduced these to a minor irritant during my real-time listening; unless you prefer the roar of a Harley to the spooky wail of a catbird, my recording was pretty useless. (Toop, 2004, p. 70)

Don Ihde recounts a similar experience with recording technology:

I go to the auditorium, and, without apparent effort, I hear the speaker while I barely notice the scuffling of feet, the coughing, the scraping noises. My tape recorder, not having the same intentionality as I, records all these auditory stimuli without distinction, and so when I return to hear the speech re-presented I find I cannot hear the words due to the presence of what for me had been fringe phenomena. (Ihde, 2007, p. 75)

\footnotetext{
45 Ian Bogost considers such intentionality with regard to the camera in Alien Phenomenology or What its
} Like to be a Thing (2012: 35-59) 
This is an important point to make. As Ihde suggests, the phonograph does not distinguish between "fringe" and any other kind of auditory stimuli, it simply records sound waves as they move a microphone's diaphragm. This reveals an important factor when considering the role of the composer of environmental sound compositions: recording technology may not necessarily represent the desired balance of sounding objects and their spatialisation as heard by the composer in situ, as the examples of Toop and Ihde attest. The composer and the phonograph have an often misunderstood relationship in this regard, as the composer's intentional object is not the object recorded. The latter is in fact the recorder's intentional object, as Bogost (2012) would likely would likely suggest, and while the recording is only made possible through the actions of the composer, this is where the relationship ends. In this way, a composer who asks "have you heard my recording of the Whanganui River", might be better to ask "have you heard my recorder's recording of the Whanganui River", peculiar though such a question might seem. The editing and montaging of environmental sounds is another matter, and something I will later discuss at length, for now I would like to assert that there will always be a disparity between what a composer may wish to record, and what is actually recorded at this basic technical level. As a composer with environmental sound I have encountered this phenomenon on several occasions, and I would now like to detail one particular case.

\section{BFMTPHNO-004 (2015)}

This piece explores the 'intentionality' of the phonograph. As with a number of my ambisonic works, this piece comprises a single take recording of an electronic improvisation performed within the environment. In this particular instance, the improvisation takes place along a shoreline, using four loudspeakers, two hydrophones, and digital processing. Towards the end of this piece, the audio technology used begins to perform in an unintended manner; a number of digital artifacts are introduced to the recording as the system runs low on power; though they are easily identified in the recording, I was unable to detect them during the recording, as a result of the signal path employed. The specificity of 
the equipment malfunction and the resulting phonograph are detailed in Volume II, but here I will note that the malfunction is very significant from a poietic perspective. My primary intention had been that the recorder would spatially and temporally frame my improvisation, while making the experience repeatable in an acousmatic context. Though these things are achieved in the recording process, the phonograph itself imparts its own sonic signatures despite my poietic intentions. When I listened back to this piece, my initial reaction was that it had been ruined by the phonographic artifacts. However, on repeated listens I discovered that the artifacts were adding a great deal to the experience. From an esthetic perspective, it is difficult to determine where the artifacts are introduced in the signal chain, a thus what purpose they might serve. For example, a listener may hear the artifacts as generated by the composer; produced during the original improvisation, or added in post-production. They may hear them as unintended, born of the technology employed. Many of my pieces examine the ambiguity created between live improvisation and phonographic capture, and in this piece, the addition of the phonograph's intentionality extends this ambiguity, and in the process, makes explicit a crucial aspect of phonographic capture: though a composer may saddle a recording with their own intentionality, the recording has its own intentional frame, which may not always align with that of the composer.

This fissure between the composer and their recorder is born of a very important, yet under-examined condition; field recorders deal with sound only. Composers, as hard as they may try to cognitively focus on sound alone in situ, are in fact dealing with four other senses. Their experience of a sonic environment is additionally informed by all of these factors: sight, smell, touch and taste. In my opinion, a sonic environment can only be experienced as exclusively sonic when these other senses are nullified. In other words a soundscape can only be fully appreciated as such when appreciated remotely, through mediated abstraction, preferably in an environment that limits other sensorial experience, such as through headphones, or with dimmed lights in a studio. This idea finds much support in the ideas of Tim Ingold, whose ideas I will return to shortly. 
Having provided an overview of musicalised or 'aestheticised' situated listening earlier in this chapter through the writing of Fisher, as a way to distinguish between what a listener hears and what a phonograph 'hears', I will now explore the nature of listener intentionality more deeply and in phenomenological terms, so that we might see how all senses affect our understanding of the sonic environment, though we might conceive of it in strictly aural terms. Such an exploration exposes the difficulties inherent in a composer's attempt to convey their intentionality through the phonograph to a listener, as not only does a phonograph present its own intentional object, it is unable to translate the other aspects of the listener's experience (sight, touch, taste, smell), which constitute pertinent hyletic data in the noetic generation of the sonic environment.

\section{Listening as reflexive engagement with self and environment}

For now I will consider a phenomenology of sound with specific regard to environmental sound composition as Salomé Voegelin does in Listening to Noise and Silence (2010). Voegelin's book is especially pertinent to this discussion in that it addresses both situated and mediated listening practices. To begin, Voegelin makes this assertion:

Every sensory interaction relates back to us not the object/phenomenon perceived, but that object/phenomenon filtered shaped and produced by the sense employed in its perception. At the same time this sense outlines and fills the perceiving body, which in its perception shapes and produces his sensory self. Whereby the senses employed are already ideologically and aesthetically determined, bringing their own influence to perception, the perceptual object and the perceptual subject. (2010, p. 3)

Voegelin's listening is understood as engaging not only the object, but also the self. As such, listening, for Voegelin, produces both a Husserlian noema and bodily awareness. Voegelin's appraisal of perception is without doubt indebted to Husserl and Ihde, but placing further emphasis on bodily awareness, which aligns her approach with the phenomenology of Merleau-Ponty, a phenomenology that includes the notion of embodied cognition, in which the body itself is understood 
as a filter through which hyletic data must pass through. This kind of phenomenology is echoed by anthropologist Tim Ingold, who insists that sound is not 'the surfaces of the world in which we live', that is, a series of sounding bodies, but rather the medium within which we perceive:

Sound, like breath, is experienced as a movement of coming and going, inspiration and expiration. If that is so, then we should say of the body, as it sings, hums, whistles or speaks, that it is ensounded. It is like setting sail, launching the body into sound like a boat on the waves or, perhaps more appropriately, like a kite in the sky. (Ingold, 2007, p. 12)

This understanding of sound positions embodied cognition at the centre of aural experience. It follows then that composer intentionality is not informed by the 'surfaces' of sound, surfaces that may arguably be detected through the phonograph, but rather composer intentionality is informed by the ensounding of the composer; a unique experience that merges bodily awareness, past experiences and attentional focus. The composer's embodied cognition is necessarily absent in the recordings they make, and therefore this aspect is unavailable to a listener, though a composer may (unconsciously) believe otherwise. Voegelin provides an account of her experience at Waterlow Park that illustrates the extent to which her past experiences play into her noetic processes:

To listen to Waterlow Park at dawn is to generate its morning-park-ness and my morning-self from the midst of its sounds. I merge the city hum with the fresh bird song, the occasional dog walker's call and a jogger's panting breath with the sounds of my auditory imagination for which I cannot name a source. The bird's song, the traffic hum, the runner's breath and the master's whistle recall a sonic objectivity as a residue of all my earlier subjective generative appreciations of such sounds. The objective brings with it the park as cultural notion, and all the parks I have ever visited. Intertwining in my ears this left over objectivity with my present subjectivity the sounds are produced beyond what they are in a fantastic but plausible reality of what I have them to be. (2010, p. 13) 
Voegelin's account of this situated listening experience highlights how profoundly different listening experiences are between people. As such, Voegelin's 'morningpark-ness' is not only different from that of other park-goers, but it is thoroughly unique to her. Though her account of listening is compelling, the question of whether an individual can in fact meaningfully perceive mono-modally in situ is not addressed. Certainly we can focus our attention on one sense more than another, but to what extent do our individual senses inform each other? In other words, how much is Voegelin's aural 'morning-park-ness' informed by its visual counterpart? In my opinion, Voegelin's morning-park-ness is informed as much by her other senses, sight, smell, taste and touch, though she may be attending to the aural.

Anthropologist Tim Ingold might agree with my assertion. He writes:

\begin{abstract}
The environment that we experience, know and move around in is not sliced up along the lines of the sensory pathways by which we enter into it. The world we perceive is the same world, whatever path we take, and each of us perceives it as an undivided centre of activity and awareness. For this reason I deplore the fashion of multiplying scapes of every possible kind. The power of the prototypical concept of the landscape lies precisely in the fact that it is not tied to any specific sensory register - whether vision, hearing, touch, smell or whatever. In ordinary perceptual practice these registers cooperate so closely, and with such overlap of function, that their respective contributions are impossible to tease apart. (Ingold, 2007, p. 10)
\end{abstract}

Hildegard Westerkamp's soundscape work Kits Beach Soundwalk (1989) pulls this idea into sharp focus. The work is often cited as an important work of the genre, and has been included in many discussions of sound art, including Voegelin (2010) and Labelle (2007), with a more focused analysis made by Kolber (2002). Kits Beach Soundwalk makes use of recordings made at central Vancouver's Kitsilano Beach, with narration from the composer. Westerkamp begins by detailing the atmospheric conditions, geographic location and time of year. She then draws attention to the technology in use, and in doing so reveals a great deal about her situated listening experience: 
I could shock you or fool you by saying that the soundscape is this loud [increases the volume], but is more like this [decreases the volume]. The view is beautiful, in fact it is spectacular, so the sound level seems more like this [decreases the volume]; it doesn't seem that loud. But I'm trying to listen to those tiny sounds in more detail now. Suddenly the background sound of the city seems louder again [increases the volume], it interferes with my listening. (Westerkamp, 1989)

In this piece, Westerkamp illustrates the impact sight has on her aural experience, using narration and post-production in order to sonically communicate this sense to her listeners. In many respects, Westerkamp's Kits Beach Soundwalk is not only testament to the idea that aural experience is informed by other sensory or hyletic data (to use Husserl's term), but that such experience can only be promoted through the words of a composer, be they written in liner notes of a CD or program notes at a concert, or through direct narration within the work itself. Had Westerkamp's narration been absent from the piece, a listener would simply hear the volume of the field recording increasing and decreasing at various times. This leads me to a fundamental and obvious point: without written or oral contextualisation, field recordings do not have the power to impart the site's non-auditory features, let alone a composer's intentionality, even when overt manipulations have been used in an attempt to illustrate such conditions. The situated experience of the composer remains with the composer, while eluding the non-situated listener. While Westerkamp seems acutely aware of this fact, as Kits Beach Soundwalk might suggest, other composers may be less aware of it, believing that the phonograph affords a listener much more than it does. It seems very likely that this is due to a composer's ability to reconnect with their own situated experience upon playback, and an unconscious belief that other listener's may also even though many if not all listeners will lack the specific situated experience to make this same connection. Memory, then, is a very important aspect of listener intentionality where the phonograph is concerned for both a composer and noncomposer alike, and as I will discuss in chapter six, it contributes strongly to a very specific kind of engagement that is unique to the phonograph. For now, to 
see how the situated experience of a composer and their memories of it influence their compositions, I would like to consider an example.

Environmental sound composer Peter Cusack has published a book entitled Sounds From Dangerous Places (2012), which includes 34 recordings (among others) made in and around the exclusion zone of Chernobyl, the site of a major nuclear power disaster in 1986. Though the book serves to contextualise his field recordings as made in 'dangerous places', many of the recordings do not impart a sense of this danger in their own right. An example of this can be heard in his field recording "Dawn chorus, Chernobyl town". The recording reveals the cacophony of dawn birdlife without a hint of the danger that he may well have experienced in situ. Much like Westerkamp, Cusack uses further contextualisation, in this case a book and other, more explicitly 'dangerous' recordings (including environments with beeping radiometers), to connect the audience with his intentionality. In this way the dawn chorus may become dangerous with the knowledge of where it was recorded.

Let us now return to Voegelin's assertion that Waterlow Park, as it intermingles with her past experiences, 'generate[s] its morning-park-ness and my morningself from the midst of its sounds' (2010, p. 13). Had an audio recording been made of this experience, Voegelin may again experience 'morning-park-ness' from such a recording, but perhaps with additional experiences that other listeners are not privy to; the cold of dawn, the smell of flowers and the sight of London peeking above the tree line. The reconnection of a composer to their situated experience through a field recording may happen to varying degrees from person to person, as Carlyle and Lane reveal in Into the Field: the art of field recording (2013). In this book, 18 environmental sound composers are interviewed about their practices. Jana Winderen, when asked if she would consider using field recordings in her work that she did not record, has this to say:

So much would be lost if I were to do that - I'd have no sense of the temperature, or of the weather, or how accessible the place was or what the 
skipper of the boat or the local fisherman told me about the sites. All of that adds to the story I am trying to tell (Winderen in Carlyle and Lane, 2013, p. 156).

Winderen's connectedness with situated experience appears crucial in the production of her compositions, and yet without direct access to the kind of geographic and atmospheric conditions, local stories or any other non-auditory information, a listener is unlikely to hear her stories as she intended, most likely making up their own or disregarding narrative altogether. With a degree of irony, Winderen's reluctance to use other people's recordings for composition is born of her disconnection from the source environment; the exact experience that others may have when hearing her work. Other composers may not be focused on engagement with the non-auditory as Winderen appears to be, though such senses undoubtedly influence their decision-making during recording and postproduction. For these composers, while non-auditory senses may sit tacitly in the background in situ, so too may they tacitly reemerge upon audition. In fact, a recording may act as a memory trigger not just for other sensorial information, but also for the total embodied experience. Winderen's comment above attests to this idea. I believe this experiential transition from the composer in the field to the composer listening back in the studio plays a key role in many works of soundscape composition. The belief that more than just auditory information can be conveyed through sound is the crux of yet another conviction: that field recordings have the power to educate a listener about an environment. This is an idea I will discuss at length in chapter four.

As Winderen's comments suggest, it is also true that a composer may find their recording disappointing (just as Toop and Ihde expressed) and such a lack of reengagement with the fullness of in situ experience may lead to specific types of manipulation of the recording, as Christina Kubisch recounts:

When you are recording it heightens your perception and you become very sensitive to everything around you, it becomes an incredibly intense experience. Then when you listen to your recordings afterwards in the studio you're often disappointed because it's just the sound and not the 
experience and so you have to change and rearrange the material in order to bring back that original sensation. (Kubisch in Carlyle and Lane, 2013, p. 70)

Kubisch's comment is revealing, and while Westerkamp's Kits Beach Soundwalk may not have been written out of disappointment, there is a commonality here in production methodology. In my experience, a composer may be 'often disappointed' but not always disappointed. I have had both experiences with my own recordings, and I believe this is probably true for many; while one recording may magically re-create 'the original sensation' or extra-auditory context for the composer, another recording may not: the composer hears only the 'second musicalisation' of the recorder on playback, that is, the environment recorded without the composer's situated intentional bracket (the first musicalisation). What is revealed here is this: to varying levels, a composer may engage with situated experience upon audition of a recording. I believe that even at the formalist end of the spectrum, where we might find the compositions of Francisco Lopez, a composer will engage with elements not present in the sonic material; though Lopez may champion an environment's capacity for self-referentiality (1997), other references may influence his works. In other words, though a composer like Lopez may feel no desire to recreate situated experience in their work, focusing instead on the acousmatic result (1997), I believe that compositional process alone, that is, the activities surrounding the act of recording, may not only be enough to engage a composer with the past and influence their decision-making, but may also be a deeply engaging and motivating factor in the compositional process. As such, while many composers, like Westerkamp, undoubtedly find the ecological or narrative context ascribed to the environment to be a motivational force in their works, others may find the fuller context of field recording itself to be motivational. Davide Tidoni might agree. He notes this of some drum kit recordings he made in his basement as a teenager:

On a very biographical level, those tapes bring me back to the emotional feelings and relational context under which I was recording. Those recordings are not only about what is taped itself... but actually concern 
what happens before and what happens after (Tidoni in Carlyle and Lane,

2013, p. 76)

On a personal level, a composer may feel the recordings that elicit those emotional and situational responses within themselves are the most suitable for selection; setting compositional motivations aside, composers are always more invested in these recordings by definition. Others may be led to believe, perhaps without conscious acknowledgement, that the feelings and experiences that a recording provides them with will somehow materialise for others; a kind of collapsing of experience from composer to listener: a belief that my morningparkness is your morning-parkness. Such a belief doesn't weather much scrutiny, but, as I will argue, this misconception is prevalent in a number of environmental sound composition practices.

\section{Abstraction and phonography}

What is revealed in the exploration of various attitudes to recording and the nature of experience upon replay, is that composers have differing attitudes towards phonographic abstraction; that is, the relationship between the past event and its recording. I indicated in chapter one that abstraction is a core feature of the phonograph and as such, it is important to reveal common viewpoints regarding its nature within the context of environmental sound composition in order to better define the complex relationship between the genre and this essential feature. Interestingly, two theorists closely connected to environmental sound studies, composer R. Murray Schafer and musicologist Steven Feld, have contributed two very important ideas regarding abstraction and the phonograph. R. Murray Schafer's influence within the genre of environmental sound composition is of course far-reaching, and as such, I will later be exploring many of his ideas with regard to acoustic ecology and soundscape composition. In the context of this discussion, I will be focusing on his concept of schizophonia, along with Feld's notion of schismogenesis, with regard to untransformed field recordings. 
Many composers who work with untransformed field recordings identify with the genre of phonography. The history of the genre may be traced back to works prior to Ferrari's Presque Rien No. 1 (1970). Pauline Oliveros made a 48-minute recording of the San Diego Zoo in 1968 that was aired on radio station KPFA's World Ear Project in the early 1970s. ${ }^{46}$ Walter Ruttmann's Wochenende (1930) has elements of phonography, though it is a heavily edited piece. Though the history of phonography is a rich and fascinating area of research, I can here only note that it is a practice undertaken by a large variety of people, evident in the vast number of recordings currently available for audition on sites including soundcloud.com, bandcamp.com, freesound.org, along with the more targeted sites such as those operated by labels including Touch (UK), Gruenrekorder (Germany), Green Field Recordings (Portugal), and Sub Rosa (Belgium).

Isaac Sterling, a phonographer and contributor at phonography.org summarises well the attitudes of those who identify with the practice:

The simple answer is that phonography (literally "sound writing") refers to field recording. This entails the capture of any event that can be reproduced and represented as sound. Auditory events are selected, framed by duration and method of capture, and presented in a particular format and context, all of which distinguishes a recording from the original event during which it was captured. In this respect, phonography is analogous to any other form of recording. It is distinct from recording in general only to the extent that the capture of sound is privileged over its production. This bias reflects an attempt to discover rather than invent. ${ }^{47}$

Sterling's view may at first appear contradictory. He assigns authorship to the composer, in that their method of capture 'distinguishes a recording from the original event'. At the same time, he insists that phonography exhibits a bias to 'discover rather than invent', which moves authorship away from the composer to the event itself. This particular emphasis recalls Goehr's Platonist view of the work, in which she states 'to compose a work is less to create a kind, than it is to

\footnotetext{
46 Richard Friedman and Charles Amirkhanian established the World Ear Project at Berkeley's KPFA, a radio program that requested and played hundreds of field recordings from its listeners from all over the world. The first series ran for 3 years from 1970

47 Sterling, Isaac: “What is Phonography?” (http://phonography.org/whatis.htm, accessed 14/4/2008)
} 
discover one' (1992, p. 14), though it is unclear to me if Sterling's conception of the work in phonography is truly nominalist. Regardless of where Sterling believes authorship and the work reside in phonography, his statement recognises the two essential features of the phonograph that I argued for in chapter one. Abstraction is noted in the distinction between the recording and the original event, while documentary is noted in the desire to discover what is nonetheless captured, albeit as a trace of the past event. I will discuss the notion of the 'trace' in greater detail in chapter four, but here I will reiterate that though the event itself is not present in its recording, aspects of it are embedded in the phonograph as traces of a past reality. This duality of documentary and abstraction in the phonograph does not sit so easily within the more dogmatic practice of soundscape composition, or in acousmatic music practices more generally: indeed it is the pitting of documentary against abstraction that is often the very source of the tension between formalism and mimesis in electroacoustic works. ${ }^{48}$ The difficulty in acknowledging and accounting for abstraction and documentary in field recording is evident within the two predominant conceptions of abstraction promoted within environmental sound composition.

To begin with, I would like to examine phonographic abstraction as the reduction of multi-modal experience to a single mode (the aural mode), to be experienced at a later time, and often at a different location. R. Murray Schafer coined the term 'schizophonia' to describe this phenomenon:

The Greek prefix schizo means split, separated; and phone is Greek for voice. Schizophonia refers to the split between an original sound and its electroacoustical transmission or reproduction (Schafer, 1977, p. 90)

Schafer intended this term as a 'nervous' word, designed to highlight and promote his personal anxiety concerning recording practices. For Schafer, sounds have been violently split ('torn') from their source ('natural sockets') and

\footnotetext{
${ }^{48}$ Denis Smalley in particular has highlighted this tension in a number of papers. See "The Listening Imagination: Listening in the Electroacoustic Era", Contemporary Music Review, Vol. 13, Part 2, 1996, pp. 77107. Veit Erlmann's Reason and Resonance (2010) provides historical context for this tension.
} 
given independent existence. Given the emotive language used, we must conclude that for Schafer, 'original' sound holds a kind of moral authority over its reproduction. As Steven Feld notes (1994, p. 259), the notion of schizophonia returns us to Walter Benjamin's assertion that 'what withers in the age of the technical reproducibility of the work of art is the latter's aura' (Benjamin 1939/2003, p. 254). In many respects, Schafer ascribes Benjamin's status of the 'original' artwork to the sonic environment, with recordings of such environment's lacking the 'aura' of the original.

Stripping away the emotional connotations of schizophonia for a moment, we are left with a basic conception: an environment and its recording are distinct from each other. As environmental sound artist Francisco Lopez points out, 'schizophonia and objet sonore [sic] are antagonistic conceptions of the same fact' (Lopez, 1997). In this respect, Lopez sees the splitting of a sound from its original source as a starting point for a new music in Schaefferian terms. While R. Murray Schafer laments the environment/phonograph divide, Lopez promotes it. Regardless of their personal attitudes towards abstraction, there can be little doubt that both Lopez and Schafer see abstraction as the fundamental feature of the phonograph, to the point where the notion of documentary is eclipsed by it. That is not to say the documentary power of the phonograph is not acknowledged: Lopez in particular sees documentary as an aspect to be muted in order to attend to the abstract, a notion that is indebted to Schaeffer. R. Murray Schafer, however, is less clear about the presence of documentary in recordings. What is clear, is that in no point in The New Soundscape (1973) does he acknowledge documentary in schizophonia.

While l'objet sonore and schizophonia respectively celebrate and deplore the role of the phonograph where environmental sound is concerned, others are less convinced of the void it purportedly creates. In response to Schafer's schizophonia, anthropologist and ethnomusicologist Steven Feld adopted a new phrase, schismogenesis, in an attempt to reframe this void. As Feld recounts in his paper "Schizophonia to Schismogenesis: On the Discourses and Commodification 
Practices of "World Music" and "World Beat” (1994, p. 257-289), Gregory

Bateson describes schismogenesis as:

classes of regenerative or vicious circles...such that A's acts [are] stimuli for B's acts, which in turn [become] stimuli for more intense action on the part of A, and so on. (Bateson in Feld, 1994, p. 265)

As Bateson's description suggests, Feld envisages the divide created by the phonograph as not a simple transitive and finite relationship from original to recording as Schafer does, but rather a cyclical relationship in which the recording feeds back to the original, and so on. Feld's paper argues this concept with regard to the relationship between "world music", "world beat" and the popular music recording industry with some conviction. He also uses this concept to justify the techniques used in the production of his CD Voices of the Rainforest (1991), of the Kaluli people and the environment of Bosavi, Papua New Guinea. With this release, Feld attempts to construct a commentary on the way in which Kaluli relate to the Bosavi environment, using a great deal of editing and montaging to create an hour-long pseudo-document of a 24 hour period, omitting a number of sounds prevalent in the environment, including generators, helicopters, church bells and cassette players (1994, p. 286). As Feld insists, these techniques are not intended to be deceptive, but rather to 'amplify that world unashamedly, in the hope that hearing it might inspire and move others as it has inspired and moved me' (p. 286-287). In some respects, this kind of production recalls Albini's recording and mixing methods as explored in chapter one, in which the mix is used to exaggerate the live experience, though where Albini strives to heighten punk authenticity through production methods, Feld introduces a level of fantasy; his is certainly not a "warts and all" approach: the "warts" of Bosavi have been removed. Feld here provides an interesting outlook. On the one hand, he promotes an understanding of recording as cyclical, and yet he goes to great lengths to instate a clear division between environment and recording through editing, and most importantly, omission. In the omission of certain features, Feld displays a specific intentionality regarding his personal aesthetics: some sounds are more appropriate for promotion through recording 
than others. As I will further explore in chapter four, this particular intentionality is common among soundscape composers. Soundscape composers, while indebted to Schafer for his ideas around acoustic ecology and the soundscape more generally, do not always share his ideas surrounding the recording of these. It would seem that many soundscape composers believe in a recording's power to translate environmental context into a phonograph in a form that schizophonia refutes, as Schafer clearly believes that schizophonia instates a divide that strips a recording of its contextual significance, and thus its value. However, given Schafer's use of the phonograph in his educational endeavors, it is likely that he used the term schizophonia to level a criticism at the technological operations of mass culture while tacitly understanding the relationship between environments and recordings in ways that are akin to Feld's schismogenesis, for at the very least, Schafer believed that recording held the power to educate, thus creating a feedback loop from the recording of an environment to the environment itself. In short, though Schafer denied the phonograph its documentary power through schizophonia, he nevertheless exploited this very feature in his educational endeavors. Keen to provide the public with this perspective on the soundscape, The World Soundscape Project, with Schafer at the helm, released a double LP entitled The Vancouver Soundscape (1973), with the final track “On Acoustic Design” functioning as 'an introduction to the science and art of composing the soundscape, narrated by R. Murray Schafer, with recorded examples of good and bad acoustic design in Vancouver'49

Irrespective of the finer points of the preceding discussion, two strong conceptions of abstraction emerge in the discourse surrounding environmental sound recording. The first, represented by schizophonia, is that no integrated relationship exists between an environment and its recording. The recording, though abstracted from the environment, remains ontologically distinct. Lopez makes this claim:

${ }^{49}$ See http://www.sfu.ca/ truax/vanscape.html (accessed 24 September 2013) for a full description of the album's contents. 
I am professor of Ecology and I have been recording and composing with sound environments since more than fifteen years ago. Although I am quite aware of the obvious relationships between all the properties of a real environment, I think is an essential feature of the human condition to artistically deal with any aspect(s) of this reality. (Lopez, 1997)

Lopez clearly has a very distinct intentional perspective. In situ, he has a specific set of experiences that enable him to constitute the environment from the perspective of an ecologist, though this is truly an oversimplification of what is undoubtedly a multi-faceted engagement. In terms of his artistic intervention, Lopez deals with the aural 'aspect of this reality', by recording it, and insisting that such a recording has 'the full right to be self-referential' (Lopez, 1997). As such, Lopez seeks no recourse to the environment through recording: he considers the two as ontologically distinct. In essence, Lopez acknowledges and champions the singular and irrefutable ontology of the phonograph, yet, he does so at the expense of another. As I will discuss shortly, Lopez employs a very similar methodology to Pierre Schaeffer in promotion of abstraction, one in which the documentary power of recorded sound must be muted.

The second reading of abstraction promotes a reflexive relationship in which environments (or at least responses to them) are informed and modified by their recording. Such a relationship seems plausible in instances where the intentional object of an event is understood in broad terms: a jazz recording may promote new practices in jazz performance, for example. ${ }^{50}$ However, in instances where an event is considered tied to its original temporal expression (such as a specific concert), or where the event recorded lacks human intentionality (such as an environment), schismogenesis seems less applicable, given the inability of a listener's present intentionality to modify past events.

Both of these conceptions are problematic. To begin with, though schismogenesis acknowledges the documentary power of the phonograph through the promotion of another power; that is, the power of recordings to modify potential future events, it does not address the actual relationship that

50 Mark Katz dedicates a chapter of his book Capturing Sound (2010, p. 80-94) to this subject. 
exists between the moment of capture and the moment of audition. Simply put, schismogenesis recognises the phonograph's documentary feature in general, but not specific terms. Schizophonia is also problematic, insofar as it emphatically promotes abstraction over documentary, at times without acknowledging the latter feature: acknowledgement only comes in attempts to silence it, by focusing the audience away from what is nonetheless present. Lopez uses a number of tools to sharpen this focus, including stripping his works of context by assigning the prefix "Untitled" to many of his recordings, followed by an identifying number. He also blindfolds his audiences at his concerts to remove visual referents. These techniques might be read as designed to support Schaeffer's reduced listening, insofar as they remove contextual and visual information, further focusing his audience to the specificity of the phonograph and a the notion of abstraction. However, the success of Lopez' methodology is entirely dependent on the intentionality of the listener; though he creates a situation sympathetic to the apprehension of the phonograph-as-work alone by stripping contextual information (as described above), this is not to say listeners will appreciate his compositions as such: the recording's documentary feature may persist. We might here recall Boulez' criticism of musique concrète on this basis (see chapter two).

To illustrate this point, I would like to consider Lopez' vivid work La Selva (1997). La Selva is a reserve in Costa Rica, and at a technical level, La Selva (the work) is a recording of this reserve. Lopez has this to say of this relationship in the liner notes of the CD:

What you can listen on this CD is not La Selva; it explicitly doesn't pretend to be so. In other words, La Selva (the music piece) is not a representation of La Selva (the reserve in Costa Rica). It certainly contains elements that can be understood - and even used - as representational, but the essence of the creation of this sound work that I'm calling a piece of music is rooted on a 'sound matter' conception, as opposed to any documentative approach. (Lopez, 1998) 
Again, Lopez makes the distinction between the event and its recording plain. He asks us to consider his work as 'sound matter' rather than a document. ${ }^{51}$ However, when I listen to La Selva, It is not the spectromorphological contours of the recordings that I primarily attend to; rather it is the sound of a rainforest (in Costa-Rica) that I hear. That is, its source-bonded referent, perhaps as a result of my own experiences of field recording in Central America. Such a dilemma points to a phenomenological confusion presented by the phonograph: while the phonograph itself may be the intentional object (noema) for some, the original context may be the intentional object for others, though it may be an altogether imagined context. This dilemma is not lost to Lopez. As quoted above, he suggests that La Selva 'contains elements that can be understood... as representational'. What emerges is this: in order to understand environmental sound recordings as purely self-referential 'rooted on a "sound matter" conception', listeners may need to employ a degree of cognitive bracketing, in other words, a listener is asked to ignore what is nonetheless present. This, of course, is the thesis of musique concrète. The fact that this dilemma still presents itself almost 60 years after the first works of musique concrète suggests to me that the popular notions of abstraction as exemplified in schizophonia and l'objet sonore, fail to account for the phonograph as experienced by a listener. Schismogenesis, though understood as an augmentation of schizophonia, designed to address the awkward divide between the event and its recording, does so in terms that are too general. The direct relationship between an event and its recording, though acknowledged in the transition from $A$ to $B$ (in Bateson's model), is not specific enough. The specificity of this relationship forms the basis of discussions in chapter six.

I argue that responses to the phonograph are much more complex than these terms suggest. If we return for a moment to Lopez' La Selva, I, in listening to the work I may be simultaneously aware of the rainforest (contextual properties), the stereo field (spatial properties) and the harmonic relationships between sounding entities (spectral). In this way, I am using two noetic processes to

51 Lopez discusses his poietic intent in the production of La Selva (1997) in his essay 'Environmental Sound Matter' (1998) http://www.franciscolopez.net/env.html (accessed: 11/03/2012) 
arrive at a single noema (formal and contextual), or perhaps a single noetic process of one who is familiar with both reduced listening and field recording. Furthermore, my noetic processes may change over time. Where La Selva is concerned, I may begin by identifying source context, but after several minutes, my intentional focus may drift to the phonograph itself, through a focus on the formal contours of the materials. I may even perceive the work as phonographspecific during one audition, and then phonograph-as-document in another, such is the ability of my intentionality to construct through noesis given my specific set of experiences. What emerges is that neither schizophonia nor schismogenesis can entirely account for phonographic abstraction and documentary given the range of experiences a listener may have when encountering environmental recordings. However, they do present two influential conceptions of abstraction, and as I will discuss shortly in chapter four, these conceptions often play into the way in which environmental recordings are produced.

Before moving forward, it is important to reiterate some key points from discussions in this chapter. The first relates to Husserlian intentionality. To begin, Husserlian intentionality understands intentional objects as constituted in noetic perception. Importantly, this noetic perception is tied to the individual, meaning that intentional objects are different from person to person. Unlike listening to music, a practice in which the intentional object resides in the 'natural attitude' of perception, environmental listening aesthetically must employ a kind of bracketing akin to a Husserlian reduction. In Cageian intentionality, the environment is considered for its sonic or spectromorphological properties, and as Kahn has it, his intentionality is formalist in nature, deriving from the influence of absolute music. Where Schaefferian intentionality is concerned, environmental listening is framed according to its suitability for acousmatic composition, and as I have argued, this constitutes an intentionality that imagines a second, phonographic noema. Cageian and Schaefferian intentionality both seek to formalise environmental sound, albeit in different capacities, which amounts to an attempt to create an inter-subjective response among listeners. The extent to which an inter- 
subjective response to environmental sound is possible through formalism (or any other means) seems limited at best, especially given the comments of Boulez (1968) and Schaeffer himself (1986/2012), as recounted in the last chapter.

In this chapter, Fisher's appraisal of environmental listening, as he argues against a formalist evaluation of environmental sound, exposing the complexity of attentional focus to a given environment, thus reveals the unlikelihood of inter-subjective intentionality between listeners. Voegelin's appraisal of listening, which ties intentionality to embodied cognition and memory further extends the distance between listeners and their perceptions of environmental sound. Where the phonograph is concerned, it is clear that the intentionality of the composer is not transferred to the recording itself (a technology that exhibits its own "neutral" intentionality), though in some cases the composer may believe that it has transferred, due to their personal reengagement with the situated experience upon replay. Other composers, including Hildegard Westerkamp, may use composition as a way to make their intentionality explicit in light of this fact.

The variety of responses to environments and their recordings by composers reveals various positions regarding the notion of phonographic abstraction, and in turn, documentary. Schizophonia and schismogenesis are two commonly employed conceptions but both fail to account for this duality in its entirety: schizophonia denies any recourse to the source material, essentially muting, or in some cases, refuting the existence of the phonograph's documentary feature, while schismogenesis suggests that a relationship exists between past and present, but also without regard for the direct link between the past and present of the phonograph; connection to the sonic past is not made through the recording itself, but through the behavioral modifications of the listener who has heard the recording. These views of intentionality and abstraction greatly influence the production of environmental sound composition, especially concerning the role of the composer, notions concerning a work's meaning, the ability of works to convey their composer's ecological concerns, and the role of the listener. 


\section{4}

\section{Soundscape Composition, Semiology, Intention and Reception}

\section{Introduction}

In this chapter I will explore the concepts of acoustic ecology and soundscape composition as a means to examine the divide between composer intention and listener reception. ${ }^{52}$ Though this divide is easily observed in soundscape works, it is by no mean exclusive to the genre. Additionally, soundscape composition is chosen for analysis in this chapter, as it is the genre of environmental sound composition that is arguably the most ideologically prescriptive of genres with a clearly defined history. Although there are many different types of environmental sound composition, the legacy of soundscape composition continues to define the parameters within which we discuss it, and as such, soundscape composition is a very important genre to understand.

Throughout this chapter I will provide evidence that composers who create works under the banner of soundscape composition adhere to a set of ideological concerns; concerns informed by the values of acoustic ecology. Indeed, I will argue that soundscape composition is defined by these concerns. Hildegard Westerkamp (2002) is perhaps the most enduring proponent of soundscape composition in these terms, and as such, I will be referring to her writing in many instances. To observe the prescriptive nature of soundscape

52 Acoustic ecology is well defined by Kendell Wrightson in 'An Introduction to Acoustic Ecology'. See Wrightson (2001). 
composition, we need look no further that Barry Truax's Acoustic

Communication (2001), a key text of soundscape theory originally published in 1984. In his book, Truax lists four criteria in the production of soundscape composition:

(1) Listener recognisability of the source material is maintained, even if it subsequently undergoes transformation;

(2) The listener's knowledge of the environmental and psychological context of the soundscape material is invoked and encouraged to complete the network of meanings ascribed to the music;

(3) The composer's knowledge of the environmental and psychological context of the soundscape material is allowed to influence the shape of the composition at every level, and ultimately the composition is inseparable from some or all of those aspects of reality;

(4) The work enhances our understanding of the world, and its influence carries over into everyday perceptual habits. (2001, p. 240)

Though I will provide a thorough analysis of these points later in this chapter, it is important to note here that in general terms, Truax defines the way in which soundscape composers should produce works and how listeners should hear works. In this regard, soundscape composition and musique concrète display a similar kind of dogmatism. However, Truax also describes the way in which soundscape composition should influence 'everyday perceptual habits', which clearly suggests that soundscape composition should not be limited to an interaction between a composer and listener through an acousmatic work, but rather this interaction should modify the way in which a listener perceives beyond that confines of real-time engagement with the work. In other words, soundscape composition, as promoted by Truax, should educate its listeners.

Soundscape composition is a genre of environmental sound composition that has very complex attributes, not least where composer/listener intentionality and the role of the phonograph are concerned. This chapter deals with these specific attributes in detail. In order to understand the genre in simpler terms, 
we might begin by defining soundscape composition as producing a phonograph of a meta-document type, insofar as there are three aspects to attend to: that of the original event (the past), that of the composer in the studio (an intermediate past), and that of the acousmatic listening experience (the present). In this way, soundscape composition is much like dub music, in that the composer acts as an intermediary between past and present experience, and their manipulations amount to a reworking of recorded materials to provide a new, transformed context. It is this intermediary role that forms the basis of much criticism of soundscape composition: unlike dub music where the engineer is thought to engage, primarily, with the recorded materials and with a view to realise something new, soundscape composition is often thought to primarily focus on the listener, asking for a particular kind engagement without acknowledging the complexity of a listener's engagement with the materials themselves. I have addressed the notion of the composer as occupying an 'intermediate past' between the present audition and recording of materials in a piece presented in the second volume of this thesis, which I will briefly explore here.

\section{CHPHNO-002 (2015)}

The core materials for this piece were recorded in two locations. The first recording was made above the Terrace Tunnel at night; a tunnel for passenger vehicles that connects Wellington City to northern arterial highways. The second recording was made in my home studio in Lower Hutt, while I manipulated the materials collected above the tunnel. The purpose of this piece was to explore the 'intermediate past' of the composer, by recording that intermediate space of composition (my studio) and including it in the piece. I have attempted to make this 'intermediate space' explicit in the piece, which is, in part, made naturally due to the implicit contrast between the two spaces (one outdoors near a highway, the other indoor in a quiet suburban street), but also within the temporal structure of the work; the piece gently transitions from an exterior space to an interior space through the manipulations performed in the interior space, thus the connection between the two is laid bare. 
The piece begins with cars entering and exiting the tunnel. Gradually, I introduce processing, namely, the manipulation of materials through hardware reverbs, delays and comb filters. I then introduce the sound of my performance with these hardware devices into the piece. This particular recording comprises a stereo capture of my movement between equipment, flicking switches and operating machinery. As I monitored my improvisation with these hardware effects through headphones, this particular recording is devoid of the auditory results of such a performance: a listener only hears the sound of faders, toggles, spooling tape and the composer operating the equipment. The piece ends with my 'tacit' performance in the studio space, and as such, the piece makes explicit both the past of the tunnel recording, and the intermediate past of the composition's production. This particular production methodology highlights an important feature of many environmental sound compositions: though a composer might try to convey a sense of place in their pieces, the overt manipulation of materials introduces the listener to an important secondary place: the site of production. I believe that many composers, especially soundscape composers, intend that listeners focus their attention to the primary materials of the piece (as they themselves have) and that their manipulation serves to augment listener experience without detracting from these core materials. However, it is my view that such manipulation undoes the composer's poietic intentions for their listeners on the basis that manipulation introduces and intermediate past that can split the focus of listeners.

In a number of other pieces included in the second volume to this thesis, I have attempted to remove the secondary site of production from my works by collapsing the site of production to the site of recording; that is, I have taken the studio equipment into the field and made single take recordings of live improvisations with audio technology. I have already detailed one such piece BFMTPHNO-004 (2015) - in the previous chapter. Here I would like to detail another. 


\section{BFMTPHNO-001 (2015)}

This piece was recorded in Francis Bell Reserve in the hills of Lowry Bay, Eastbourne, with an ambisonic microphone. Four loudspeakers were set up in a quadraphonic array, each one diffusing a sine tone. I controlled the pitch and amplitude of these individual sine tones with a MIDI controller. The details of this improvisation are described in the second volume, but the important thing to note here is that there was no postproduction on the recording, and thus the intermediate past of production does not exist in this recording. As such, unlike many environmental sound compositions, this piece employs a methodology that not only challenges the normal production methodology of environmental sound composition, but fundamentally changes the relationship between the composer and the environment. Such a change has implications for the listener as well. If a listener is now unable to locate the composer at an intermediate past, curating the sounds at a remove from the environment itself, how might such works be understood?

Returning now to a growing opposition to the ideology of soundscape composition, Salomé Voegelin (2010) believes that works attempting to present an 'authentic sense of place, for the purpose of preserving endangered sounds and fostering acoustic awareness' have the effect of 'producing a more didactic composing at the listener, to make him hear' (p. 32). Likewise, Francisco Lopez, in opposition to such didactic composing, implores that 'a musical composition (no matter whether based on soundscapes or not) must be a free action in the sense of not having to refuse any extraction of elements from reality and also in the sense of having the full right to be self-referential, not being subjected to a pragmatic goal such as a supposed, unjustified re-integration of the listener with the environment' (1997) (I will be looking at this notion of listener 'reintegration' with regard to soundscape composition later in this chapter). Speaking on the relationship between her works and acoustic ecology, Christina Kubisch notes: 'I don't belong to the eco-faction like [R. Murray] Schafer... it's too strident and pregnant with symbolism... I don't want to make demands on the listener in advance. I want to stir something that is already in him, 
something that can be carried further on an individual basis. I don't want to direct, it's not my nature' (Kubisch in Toop, 2004, p. 78).

Regardless of whether a listener is interested in the concerns of acoustic ecology or not, a fundamental observation remains: when attending to a soundscape work, a listener perceives the composer as located precisely between themselves and the materials. In other words the presence of the composer is keenly felt as a mediator of experience, at the very least because a listener is aware that a work is composed. The listener's awareness of the composer's presence becomes more acute the greater the manipulation of materials. This is common to all soundscape composition, and yet, it seems at odds with the kinds of listening practices promoted by its composers. Comments by Westerkamp including her insistence that her works should encourage a 'journey into the inner world of listening' (Westerkamp in LaBelle, 2006, p. 209) seem to promote a kind of listening in which a new aural awareness is produced. However, it is not the journey of the listener themselves that is encouraged, in a manner akin to Pauline Oliveros' 'deep listening' (Taylor, 1993), but a directed listening, an accompanied listening, whereby Westerkamp attempts to guide listeners through her world of listening. This is most explicitly observed in Kits Beach Soundwalk (1989). It is of little wonder then that some listeners do not wish to embark on the journey, or would rather take their own, knowing their experience will be interrupted by a composer's didactic arrangements. In other words, where many soundscape composers (Westerkamp in particular) attempt to evoke a heightened form of listener awareness of acoustic ecology through composition, a very heavy-handed approach can result in listener alienation from the materials and any message that is promoted may be lost. My view is that this style of composing is problematic, not least of all because of the inherent mismatch between the intention and reception of works, as suggested by the comments of Voegelin, Lopez and Kubisch.

As the title of Will Montgomery's paper "Beyond the Soundscape: Art and Nature in Contemporary Phonography" (2009, p. 145-161) suggests, 
contemporary phonography in many ways lives in the shadow of soundscape theory and indeed soundscape composition. His paper deals with the practices of sound artists Chris Watson, Peter Cusack, Kiyoshi Mitzutani, Toshiya Tsunoda, Jacob Kirkegaard and Stephen Vitiello, none of whom identify with the soundscape movement, and each of whom have unique approaches to and understandings of the sonic environment. Just as Montgomery does, I wish to consider contemporary environmental sound composition, including my own, as removed from soundscape composition, though as stated, in order to do so, it is necessary to investigate the genre's ideological influence. In analysing soundscape composition, I will argue that though it is an important form of the environmental sound composition, it should not be considered the central practice around which all others are orientated and understood. In my view, soundscape composition has effectively colonised environmental sound composition, even if inadvertently, through its focus on acoustic ecology, and in doing so has polarised the community, as well as influenced discussions on how environmental sound composition should be understood. Francisco Lopez' paper "Schizophonia vs l'objet sonore: soundscapes and artistic freedom" (1997) is exemplary of what is now a growing challenge to the importance of soundscape theory within the genre. His paper also indicates just how pervasive the influence of soundscape theory continues to be. Though many contemporary composers seek to create new forms of environmental sound composition using various aesthetic methodologies and technical processes, the tensions stemming from soundscape composition are never far from the surface.

As I will detail shortly, some soundscape composers question the validity of phonographic works that are not clearly aligned with the concerns of acoustic ecology. The root of this attitude can be found in the views of R. Murray Schafer, who questions the artistic value of field recordings when describing his own work with the World Soundscape Project in the early 1970s:

A lot of these recorded soundscapes began to be [Schafer laughs] regarded as art. And it started actually in Germany with quite a famous German producer, radio producer, by the name of Klaus Schöning, at the West German Radio in Cologne. He played the whole of the Vancouver 
Soundscape recording as if it were a new kind of composition, a musical composition that we'd put together, and he got so excited about it that he then commissioned more city portraits. (Schafer, 2005)

Schafer continues,

However, my purpose and our purpose was not to make works of art, but simply [to] supply ourselves with a catalogue of information; sound information that we could measure (2005)

Schafer, heavily invested in acoustic ecology, may have found no artistic value in field recording, a view that is hardly surprising given his concept of schizophonia. His colleagues however, particularly Hildegard Westerkamp and Barry Truax, would find ways to bend their inherent desire to work creatively with field recordings to fit the concerns of the movement. Westerkamp describes this process, stating that soundscape composition 'can make use of the schizophonic medium to awaken our curiosity and to create a desire for deeper knowledge and information about our own as well as other places and cultures.' (2002, p. 52) Soundscape composition, as it eventually became known, would become a creative arm of, and perhaps even a promotional tool for, the concerns of acoustic ecology and soundscape studies.

As mentioned, some composers question the value of environmental sound compositions that do not reflect the concerns of acoustic ecology. Westerkamp is exemplary in this regard. In her paper "Linking Soundscape Composition and Acoustic Ecology" (2002), Westerkamp makes clear the relationship between soundscape composition and acoustic ecology, suggesting that the link between the two is what gives meaning to environmental sound composition. In her paper, she recounts the words of composer Michael Rüsenberg when describing one of his works. Detailing a particularly noisy tram station in Germany, Rüsenberg argues for a distinction between an acoustic ecologist and soundscape composer: 
The acoustic ecologist would rather do a noise level survey from different perspectives and conduct interviews with commuters. But the soundscape composer - very perversely - might discover for example, the beauty within the Doppler effect of a passing Harley Davidson and enhance the descending pitch. (Rüsenberg in Westerkamp, 2002, p. 54)

Rüsenberg displays an unusual perspective. Here, he considers himself a soundscape composer without being an acoustic ecologist. His particular interest in this environment is based in its spatio- and spectromorphological properties. Westerkamp questions this stance:

If certain aspects of a soundscape recording become enhanced through processing, what is the composer trying to say with this and how does it contribute to a deeper understanding or a renewed relationship to the soundscape or to our own listening. Or, if processing is done for the pure pleasure of it, why would the composer want to create a soundscape of noise in the concert hall?... is it not the soundscape composer's responsibility to act like an acoustic ecologist? (2002, p. 54)

Westerkamp may be correct in this regard. Many would agree that soundscape composition and acoustic ecology are forever connected through the work of Schafer and the World Soundscape Project. However, what if Rüsenberg did not identify himself as a soundscape composer? It seems to me his stance is rather less dogmatic than that of Westerkamp. In fact, his intentional bracketing finds greater commonality with Cage or Lopez. I argue that Westerkamp would level the same criticism at Rüsenberg regardless of how he perceives himself. For Westerkamp, meaning is imbedded in the materials themselves, and a composer must work to uncover them:

No matter what the composer's intent may have been from the start, the materials inevitably speak with their own language, whose deeper meanings may only emerge with repeated listening and sound processing. (2002, p. 53-54)

Here, Westerkamp articulates a fundamental tenant of soundscape composition, which I am querying: that the materials themselves intrinsically carry the 
concerns of acoustic ecology with them, somehow independently of the composer, and that a composer's role is to enhance this 'deeper' meaning through composition. This view is particularly problematic given the role of schizophonia within soundscape composition. As quoted previously, Westerkamp believes composers should 'make use of the schizophonic medium', yet this medium negates the possibility of recordings to carry such meaning. This paradox raises questions surrounding the ability of soundscape compositions to convey meaning, thus striking to the very heart of the phonograph's ontology (I will address such questions in the discussion of soundscape composition and semiology that follows this section). Regarding Westerkamp's assertion, it is difficult not to conclude that for her and those who share or support her views, environmental sound compositions that do not promote the assumedly intrinsic meanings of source materials are essentially missed opportunities and otherwise meaningless. In other words, in Westerkamp's opinion, all environmental sound compositions - soundscape composition or otherwise - have an obligation to the materials themselves to promote acoustic ecology.

In essence, Westerkamp intimates that in order for an environmental recording to have any value, it must meet a number of different criteria that typically involve the integration of her very particular, ecologically charged, intentionality with that of the listener. Though a rift appears between Schafer's previous comment (cited above), one that would seem to deny phonography any artistic merit, and Westerkamp's compositional practice, they both insist on the power of the phonograph to educate a listener about the soundscape. A key difference between the two is that Westerkamp, and soundscape composers more generally, attempt to do this through artistic intervention while Schafer does not. It is the insistence on soundscape theory that inextricably binds soundscape composition with acoustic ecology, though the perspective of artists such as Michael Rüsenberg suggests otherwise. To build a better understanding of what acoustic ecology is and the precise role it plays within soundscape composition, I will now turn to the early writings of R. Murray Schafer. 


\section{Soundscape theory and acoustic ecology}

The term 'soundscape' was coined by Schafer in the late 1960s and thoroughly discussed in his influential book The Tuning of the World (1977). In essence, Schafer's soundscape is analogous to landscape (though Ingold clearly refutes this comparison, as noted earlier). At a basic level it denotes all sounds heard in a particular environment. However, Schafer's understanding of soundscape is not this prosaic. Indeed, his detailing of it reveals a very particular stance regarding how it should be researched, comprehended and ultimately, orchestrated:

Today, all sounds belong to a continuous field of possibilities lying within the comprehensive domain of music. Behold the new orchestra: the sonic universe! And the new musicians: anyone and anything that sounds! (1977, p. 5)

With an enthusiasm redolent of an Italian Futurist, Schafer proclaimed the world a musical orchestra. Unlike Cage's environmental orchestra, Schafer's idea of a new 'orchestra' with new 'musicians' was underpinned by a new notion: that of good music and bad music. The positing of 'anyone and anything that sounds' as musicians in an orchestra, implies that 'the piece', or 'the soundscape', had the potential to be ruined by a poor performance. In fact, Schafer believed that the soundscape had already been ruined by the industrial and electric revolutions, and his mission became that of defining a good soundscape and training hitherto oblivious musicians to improve it. As such, Schafer became chiefly concerned with the removal of certain sounds from the soundscape (noise abatement) and the championing of others (preservation) in order to keep the soundscape 'hi-fi':

The soundscape is no accidental byproduct of society; rather it is a deliberate construction by its creators, a composition that may be as much distinguished for its beauty as for its ugliness. When a society fumbles with sound, when it does not comprehend the principles of decorum and balance in soundmaking, when it does not understand that there is a time to 
produce and a time to shut up, the soundscape slips from hi-fi to lo-fi condition, and ultimately consumes itself in cacophony. (1977, p. 237)

Though Schafer has acknowledged the influence Cage had on his concept, 53 it seems unlikely that Cage would share his enthusiasm for such a composition. During his career, Cage sought to diminish the presence of the composer in his compositions, instead opting for chance procedures and advocating for composing with 'indifference'. ${ }^{54}$ Schafer's view of the soundscape and its orchestration was not an indifferent one.

The work of the World Soundscape Project can be summarised as an empirical evaluation of the aesthetically pleasing and displeasing sounds found in a soundscape conducted through extensive interviews, questionnaires and audio recordings. The project also sought to classify soundscape features in terms of their communicative qualities and cultural significance, while encouraging reduced noise levels and the preservation of important sounds as identified by communities. ${ }^{55}$ Given this, the obvious criticism of Schafer's soundscape is that it makes judgments regarding the aesthetic value of sounds within the environment that have potentially far-reaching consequences. Schafer acknowledges this criticism:

Sorting sounds according to their aesthetic qualities is probably the hardest of all types of classification. Sounds affect individuals differently and a single sound will often stimulate such a wide assortment of reactions that the researcher can easily become confused or dispirited. As a result, study of this problem has been thought too subjective to yield meaningful results. Out in the real world, however, aesthetic decisions of great importance for the changing soundscape are constantly being made, often arbitrarily. (1977, p. 146)

\footnotetext{
${ }^{53}$ In his pamphlet The Music of the Environment (1973/2004), Schafer acknowledges the ideas of Cage as influential in his concept of the soundscape

${ }^{54}$ See Douglas Kahn (1999, p. 165-174) for an interesting appraisal of Cage's philosophy during the 1940s

${ }^{55}$ A detailed summary of the World Soundscape Project and its activities can be found at the Simon Fraser University Website: http://www.sfu.ca/ truax/wsp.html (accessed 3/7/12)
} 
Given Schafer's attitude towards industries that produce excessive noise, and his resolve to combat such pollution through research, education and political lobbying, he might be forgiven for subjecting the world to his own utopian attitude to the sonic environment. In my view, his work in the 1960s and 1970s to raise awareness of noise pollution and sonic preservation should not be undervalued. By way of example, the introduction of the Environmental Noise (England) Regulations (2006) prompted the Department for Environment Food and Rural Affairs to produce the Noise Mapping England website, a site which maps noise levels in urban centers across England and is intended as a resource for noise action plans for various industries. ${ }^{56}$ These kinds of local and national noise plans and resources can trace their roots back to Schafer's pioneering work of this period. Despite the widespread success of Schafer's work in raising concern regarding noise and noise pollution, in recent years a number of voices have arisen that criticise Schafer's concept of the soundscape.

The criticism that Schafer's work is too subjective is encapsulated in the statement 'distant ambulance sirens may be one person's purgatory and another's poetry' (Toop, 1995, p. 253). However, the extent to which subjectivity pervades soundscape studies has far greater consequences than this suggests. In his article "Rethinking the Soundscape" (2010), Ari Y. Kelman insists that in promoting a 'hi-fi' soundscape (one with a large dynamic range between the 'noise floor' and 'important' sounds) over a 'lo-fi' soundscape (one with little or no differentiation between noise and preferred sounds), Schafer is essentially marginalising sonic experience in urban environments. Kelman cites Sophie Arkette as taking exception to this:

to say that the urban supervenes upon the natural soundscape, and that urban sounds can be cleaned up to resemble natural sounds is to misread the dynamics of city spaces. A city wouldn't exist if it mirrored agrarian sonic space. (Arkette in Kelman, 2010, p. 217)

\footnotetext{
${ }^{56}$ The Noise Mapping England website can be found at http://services.defra.gov.uk/wps/portal/noise (accessed $7 / 05 / 13)$
} 
Just as Schafer misreads the dynamics of city spaces, so too does he misread the dynamics of rural spaces; Schafer's general description of urban environments as 'lo-fi' and rural settings as 'hi-fi' (Schafer 1977, p.43) can easily be shown to be thoroughly inaccurate. Though there are undoubtedly higher levels of what Schafer would consider 'noise' in some urban environments this is not always the case. For example, in 2006 I made a series of recordings in Tokyo, a city with the most populous Metropolitan Area on earth (more than 37 million people). Tokyo is also known for what Schafer might describe as its 'impassioned devotion to machines' (1977, p. 237): cars, electronic goods, and loudspeakers are readily observed in Tokyo, as they are in most modern cities. I made two recordings from a second floor apartment window less than 1 kilometer from the bustling Shibuya train station. ${ }^{57}$ The first recording was at night during a thunderstorm. The distal city hum was quiet compared with the sound of thunder and the light rain on the street below. Very occasionally, a car would slowly move along the street, providing a very clear acoustic image of the space below and then disappearing into the horizon of audibility. The next morning I produced a second recording from the same apartment window. The sun was out and the streets had dried. The distal city hum was a little louder than the previous night, with the addition of 2 or 3 helicopters fading in and out of earshot. Above the city hum, light insect sounds merged with the crows of Saigoyama Park a few hundred meters away. A slowly moving rubbish truck collecting non-recyclable household items provided the only proximate sound, and like the vehicles of the previous night, outlined the acoustic space below. A recorded voice played on a loop from a loudspeaker mounted on the roof of the vehicle, informing residents of which items were appropriate for collection. This phonograph reveals what Schafer might term a 'hi-fi' soundscape, without a trace of the kind of sonic confusion he attributes to hi-density, mechanically driven city soundscapes. From my considerable experience in making field recordings, I would say that though densely populated, Tokyo has a number of very quiet areas for which a Schaferian 'lo-fi' assessment is inappropriate.

\footnotetext{
${ }^{57}$ These recording are included in my Masters thesis portfolio, A Personal and Fragile Affair (2009). Available at http://researcharchive.vuw.ac.nz/xmlui/bitstream/handle/10063/1507/thesis.pdf?sequence=1
} 
In contrast to the wonderfully 'hi-fi' urban environment described above, I have heard and recorded many 'lo-fi' rural environments, in which the cacophony of natural features, such as wind, rivers, coastlines and wildlife, prove fatiguing to listen to, a cognitive outcome which Schafer's World Soundscape Project colleague Barry Truax usually reserves for urban settings (2001, p. 21). Further to this, the kind of aural repetition produced by steady waves encountered on a windy day at the beach, or the sound of river or stream rapids, can create a condition that Truax might term 'redundancy,' in which the listener simply cognitively tunes out the sound as a matter of attending to less repetitious sounds (p. 19). Such natural features may also produce steady state noise whereby the important communicational features of a soundscape, features Truax actively promotes, are lost in the din. Such sounds may also have the effect of diminishing the acoustic horizon, in which a listener's acoustic space is greatly diminished, lessening their connectedness to the wider environment ( $p$. 26). Though discussing the environment as a duality of 'lo-fi' and 'hi-fi' may draw people's attention to the acoustic features of an environment, while also providing some insight into the ways in which they listen, it is simply not accurate to assign 'lo-fi' and 'hi-fi' to urban and rural environments respectively. Likewise, to claim that the noise produced by a city despoils communicational listening, while neglecting to apply the same logic to natural noise, reveals a clear bias towards natural environments within the soundscape composition and acoustic ecology communities.

In a letter to Soundscape: The Journal of Acoustic Ecology, sound artist Peter Cusack notes that the hi-fi/lo-fi duality is 'too static - taking no account of the fluidity and ever-changing nature of soundscapes. Even cityscapes, often cited as lo-fi have plenty of hi-fi periods and even more varying ones somewhere in the middle,' and that the duality 'comes with the inbuilt moral assumption that lo-fi = bad and hi-fi = good.' (2000, Vol. 1, no. 2) Cusack makes a very important point. Schafer's hi-fi/lo-fi model suggests everyone listens with the same aesthetic ear regarding 'noise'. This is an oversimplification of environmental listening perspectives. To cite Cusack again: 
Personally, I like a good lo-fi cacophony, e.g. the London Underground which is very rich in its sonic detail. I do not mind that my aural space is reduced. Often this brings about an increase in imaginative space. (2000)

Cusack's perspective, similar to that of Sophie Arkette's, is that urban environments promote very different kinds of listening experiences to rural ones, and that acoustic ecology, in promoting a hi-fi/lo-fi duality, fails to account for the depth of urban listening, and the positive kinds of engagements that listener's may have. Drawing from Cusack's experience in the London Underground, I have created a piece that explores the notion of 'lo-fi cacophony', which I would like to describe here.

\section{BFMTPHNO-003 (2015)}

This piece is comprised of a single-take ambisonic recording of an electronic improvisation, using four loudspeakers in a quadraphonic array around the microphone. The recording was made on a hill above the industrial zone of Seaview, Lower Hutt. Just as Cusack (2000) writes of the London Underground, this environment is a lo-fi cacophony, and my improvisation looks to subtly extend this cacophony by introducing re-pitched recordings of the industrial zone back into the environment through loudspeakers. On listening back to this recording, it is difficult to describe what is being attended to. The distal ambient noise from the industrial zone is amplified and given a new spatial dimension; it surrounds the listener due to the quadraphonic diffusion in situ. Over the course of the piece, I fade out these re-pitched and spatially enveloping recordings, and the listener is left with the environment without intervention; the distal industrial zone now has spatial specificity and micro-details can now be determined. In addition to the augmentation of a lo-fi cacophony, this piece explores the meeting place of rural and urban. In my piece, birdlife can be heard due to the fairly isolated and 'natural' surrounds of the immediate environment, and they are extended into the improvisation through processing. In my view, not only does Schafer instate a division between lo-fi and hi-fi, a division that Cusack and others argue against, but in doing so, Schafer, perhaps inadvertently, 
promotes a distinction between 'rural' and 'urban'. In many countries where there are mixtures of rural and urban settings, such a distinction is not always easily observed. Even in large metropolitan areas where urban settings are readily found, the transition from urban to rural is not always distinct; instead the transition may take several kilometers, within which many unique sonic environments many be encountered that are not defined by a urban/rural duality.

\section{Mono-modality in soundscape composition}

In addition to criticisms of Schafer's subjective and sometimes poorly reasoned ideas about urban and rural soundscapes, there is an even more compelling observation made about the very nature of his understanding of sonic experience. In the 2007 book Autumn Leaves, Tim Ingold offers a phenomenological critique of soundscape without detracting from its initial significance, which Ingold emphasises:

\footnotetext{
Undoubtedly when it was first introduced, the concept served a useful rhetorical purpose in drawing attention to a sensory register that had been neglected relative to sight. I believe however that it has now outlived its usefulness. (Ingold in Carlyle, 2007, p. 10)
}

Ingold's first criticism of soundscape is one that I have already noted with regard to recording practices: that the world cannot be split or adequately perceived along individual sensory lines in situ, and as such, only a recording of an environment can render it a mono-modal sensory experience. This is of particular importance when considering the concept of the soundscape. In drawing our attention to the sonic environment, Schafer asks us to bracket sound, to pay attention to the soundscape as independent from the environment as a totality. While Schafer acknowledges this, stating that he does not want to 'forget that that the ear is but one sense receptor among many' (1977, p. 12), nonetheless he effectively disregards other senses in pursuit of raising awareness of the soundscape. The issue here is that although Schafer asks us to consider sound alone, he also asks us to consider sound as a signifier of other 
aspects of the environment, from the noisemakers that create sound waves, to the social and cultural weight he perceives such features to carry. In other words, Schafer asks us to reduce our experience of the environment to that of sound alone, and yet he wants us to experience the environment as more than just sound. This is seen most clearly in his delineation of soundscape features: soundmarks, signals and keynotes.

Schafer's keynotes are 'sounds of a landscape...created by its geography and climate: water, wind, forests, plains, birds, insects, and animals. Many of these sounds may possess archetypal significance; that is, they may have imprinted themselves so deeply on the people hearing them that life without them would be sensed as a distinct impoverishment' (1977, p. 9-10). Additionally, a signal 'must be listened to because they constitute acoustic warning devices: bells, whistles, horns and sirens' (p. 10). The term soundmark 'is derived from landmark which is unique or possesses qualities which make it specially regarded or noticed by the people in that community' (p. 10). In essence, Schafer is not so much concerned with sound as he is with sources; that is, machines, people, animals, cities, nature etc., and attitudes towards them. This approach conceives of the soundscape as a collection of sound-making objects. This raises a question: given Schafer's desire is to evaluate the cultural significance of these sound-making objects, surely enlisting the help of our other senses would only add greater value to this exercise. In other words, why strip objects back to their aural attributes if the aim is not to consider them in purely aural terms?

As touched on in chapter three, Ingold suggests that understanding sound as source-bonded promotes the misconception of sound-as-object. Ingold has this to say of environmental listening:

Listening to our surroundings, we do not hear a soundscape. For sound, I would argue, is not the object but the medium of our perception... soundscape places emphasis on the surfaces of the world in which we live. Sound and light, are infusions of the medium in which we find our being and through which we move. (Ingold, 2007, p.11) 
With an understanding of environmental sound a collection of source-bonded objects, the Schaferian soundscape fails to account for the spatial and temporal complexity of sound as a medium, and our presence within it. It also suggests an understanding of sonic perception that instates a division between object and subject: the object (the soundscape) exists outside the body, and we (the subjects) have an obligation to modify it for the better. Objectification of the sonic environment, as Ingold understands it, overlooks the 'fluxes of the medium' instead conceiving of sound as 'already precipitated out, or solidified' (2007, p. 12), thus denying it its temporal and spatial dimensions, dimensions upon which our perception of it is contingent.

Di Scipio has this to say of sonic objectification:

\begin{abstract}
This reductio ad objectum - the objectification of sound - consisted in the forming of cognitive processes that lead us to usually consider all sound as a hard object that can be moved in time and space, that does not belong to any time and to any space, a reservoir (Bestand) that can be recalled and represented independent of the contingencies of its coming into presence.

(Di Scipio, 2013) ${ }^{58}$
\end{abstract}

This objectification or 'solidification' of sound leads to an assumption that attitudes towards it can be collectivised. Ingold believes that where visual perception is concerned, a disregard for the 'fluxes of the medium' - in this instance, light - has resulted in a similar objectification. In support of this notion, Ihde recounts, 'Aristotle... notes, "Above all we value sight... because sight is the principle source of all knowledge and reveals many differences between one object and another"' (Ihde, 2007, p. 7). I assert that the solidification of sound, as promoted by soundscape theory, gives it the assumed truth-telling capacity Aristotle accredits visual objects. In other words, in the "fixing down" of the soundscape, itself conceived as a collection of sourcebonded objects, soundscape theorist believe they are able to determine value

\footnotetext{
${ }^{58}$ In the same address, Di Scipio suggests, as I do, that soundscape composers objectify soundscapes. Whereas I believe they do so as a result of attending to the source-bonded aspects of the environment, Di Scipio believes they do so through the act of recording such environments.
} 
between one soundscape and another, between lo-fi and hi-fi, between good and bad, while collectivising responses to it. As such, for Schafer, the soundscape inherently carries information about its value, independent from the noetic responses of individuals. His perspective is not one that conceives of a listener as ensounded within the medium of sound (to use Ingold's term), but rather one that separates a listener from the soundscape. Like the Aristotelian view of visual perception, Schafer's aural perception divides the subject and object, endowing the soundscape with universally perceptible qualities; qualities that he believes warrant greater attention. As I will make clear later in this chapter, the problematic notion that a soundscape has universally perceptible value is an idea that has been transposed from acoustic ecology to soundscape composition. Before exploring this further, I will discuss the perceived relationship between soundscapes, composers and recording technology in order to develop a broader context within which this transposition may be observed. I will begin with a more focused account of schizophonia within soundscape theory.

\section{Soundscape and schizophonia}

Soundscape composition embodies two very specific notions of the sonic environment and recording technology. The soundscape, as examined above, promotes the sonic environment as a collection of sound-making objects that carry social and cultural significance. This basic premise has not undergone any substantial modification through soundscape composition in which the core tenants of acoustic ecology persist, including noise abatement and sonic preservation. We might recall Westerkamp's question as evidence of this: 'is it not the soundscape composer's responsibility to act like an acoustic ecologist?' (2002, p. 54). Though Schafer's conception of a soundscape is a very specific reading of the sonic environment, it is not a difficult concept to grasp, due in part to its over-simplification of the sonic environment and the interpretation of it. However, as I suggested in chapter three, Schafer's schizophonia is a more problematic concept, and its understanding within soundscape composition is equally so. 
To summarise the paradox of Schaferian schizophonia: it denies a recording the power to capture and convey any source context, and yet recordings of soundscapes by the World Soundscape Project are used as a research material to categorise and measure what amounts to contextual information. This points to the inherent contradiction of schizophonia - it conceives of documentary and abstraction as mutually exclusive, championing abstraction (albeit in a negative light) yet unable to shake off what is nonetheless present, the trace of a past reality in the recording. Having identified this contradiction, the question that must be asked is this: how much information - beyond sound alone - do the promoters of schizophonia believe survives the recording process and what is the nature of this information? Does a soundscape as an ecologically framed concept, in fact survive schizophonia, a notion which Westerkamp appears to support? In order to dig further into attitudes towards abstraction and documentary in soundscape composition, I will now turn to the ideas of Barry Truax, one of the genre's most prolific composers and theorists.

In Acoustic Communication (2001), and as recounted in chapter one, Truax refers to a traditional conception of the phonograph as a 'black box' and the linear progression of materials from source to phonograph to listener as 'the black box model of electroacoustics':

\footnotetext{
The "black box model of electroacoustic communication... completely ignores the fact that the context of the original source and the reproduced signal are entirely different. The concept of fidelity puts the emphasis on the quality of the signal, and therefore completely ignores the fact that there can be no "fidelity" in context between the original and the reproduced sound. (p. 11-12)
}

Such a statement clearly endorses Schafer's schizophonia concept, promoting the basic premise that recording technology is unable to convey contextual information, and though Truax uses less emotive language than Schafer, both composers impart the strong sense that the source of a sonic environment is paramount, and that recordings of these fail to deliver this original context to a 
listener. Truax believes it is then the responsibility of the composer to reinstate the source context through composition:

\footnotetext{
Although one may become more intensely aware of out-of-context sounds simply because they are isolated and framed by technological intervention, the composer is left with the problem of how to reconstruct a meaningful utterance with them. (2001, p. 227)
}

Truax proposes that although the act of framing can make a listener 'more aware of out-of-context sounds', a composer must 'reconstruct' meaning. However, the question must be asked: if Truax believes that he can reconstruct meaning through composition, how can this happen if he believes the materials of composition are inert; that is, without recourse to the original context? Put another way, how can the use of montage, granular synthesis (for which Truax is renown) or any other manipulation or arrangement 'reconstruct' meaning from materials that are without contextual substance? If this is thought to be the case, then it follows that Truax could reconstruct the source context of an environment using any materials, as it is the syntactical arrangement of sound, not the materials themselves, that provide a listener with context. This is demonstrably not the case. I contend that Schafer, Truax and the practitioners of soundscape composition must believe that an environment's source context survives the recording process, refuting the logic of schizophonia. Further to this, Truax' notion that meaning can be reconstructed through composition suggests a situation whereby a composer can draw context from the materials, as if the process of recording codifies source context, and the composer has the power to decode and present it through studio-based transformation. This understanding of the nature of phonographic abstraction and what composition can achieve is not logically sound, yet it is a core principle of soundscape composition. Further evidence of this can be seen when examining Truax' description of what defines a soundscape work, and as such I will now return to his four-point criteria for soundscape composition:

(1) Listener recognisability of the source material is maintained, even if it subsequently undergoes transformation; 
(2) The listener's knowledge of the environmental and psychological context of the soundscape material is invoked and encouraged to complete the network of meanings ascribed to the music;

(3) The composer's knowledge of the environmental and psychological context of the soundscape material is allowed to influence the shape of the composition at every level, and ultimately the composition is inseparable from some or all of those aspects of reality;

(4) The work enhances our understanding of the world, and its influence carries over into everyday perceptual habits. (2001, p. 240)

Further to this Truax concludes that 'the real goal of the soundscape composition is the reintegration of the listener with the environment in a balanced and ecological relationship' (p. 240-241). Truax assumes that listeners will not only recognise sounds, but that they will have 'knowledge' of the soundscape's 'psychological context' and that this knowledge will be brought to bear on the decoding of the composition's meaning, as presented by the composer, and that this meaning will not only enhance a listener's understanding of the world, but that it will change their perception of it. Furthermore, Truax intimates that the materials of composition must present a listener with 'psychological context' independent of the composition, as it is the meeting of this psychological context with the composition that 'complete[s] the network of meanings.' In short, Truax, like Westerkamp, endows the soundscape with intentionality (the 'psychological context of the soundscape material'). This assignment of intentionality is made possible through the 'solidification' of the soundscape, in which the source-bonded sounds are objectified. Furthermore, Truax implies that the listener will have knowledge of this context, presumably because it is thought embedded in the phonograph and made explicit through composition. In my view this is problematic and presupposes how an audience will attend to not only the materials of composition, but also the composition itself.

Embedded in the aims of soundscape as Truax understands it are two fundamental beliefs. The first is that through composition, people can be 
reintegrated with the environment, presumably a better, more hi-fi environment than abhorrent lo-fi environments. Secondly, soundscape composition is believed to be a vehicle for the concerns of acoustic ecology, and as such soundscape works should seek to spread awareness of the movement by enhancing sonic awareness of the world and changing 'perceptual habits'. To reiterate a point made at the beginning of this chapter, composers of soundscape works have a duty to educate. We might recall once again Westerkamp's notion that environmental sound composers have a 'responsibility to act [as] acoustic ecologist[s]' (2002, p. 54), and the aversion to such a directive as observed in the comments of Kubisch (Toop, 2004), Voegelin (2010) and Lopez (1997) in particular. We might also recall Katz' appraisal of classical music recordings in early $20^{\text {th }}$ century America as detailed in chapter one, in which he discusses the perceived power of recordings to 'foster positive social change' (2004, p. 49). The promoters of soundscape composition also perceive this power in environmental sound recordings, a power that constitutes the basis upon which the notion of listener education through composition is built.

My criticism of soundscape composition is that it fails in its aims to educate listeners about the concerns of acoustic ecology on the basis that those who find themselves receiving a composer's message must have already situated themselves within the context of acoustic ecology. My view is that if the success of a soundscape composition is dependent upon the establishment of composer/listener relationships in the manner prescribed by Truax, then these relationships must be established before the composition is heard, thus defeating the genres' educational aspirations. To support this critique I will first analyse soundscape composition on a semiological level, as soundscape composers deal with signification and meaning as core elements of their works, before turning to the ideas of Jacques Rancière, which I will use to examine the role of education within the genre. 


\section{Semiology and soundscape composition}

Given that soundscape composers appear preoccupied with meaning and that the primary focus of their work is to impart it to an audience, a semiological evaluation is appropriate. Here I will utilise aspects of semiology to examine soundscape composition, further developing the notion of a disjunction between intention and reception where field recordings are concerned. This disjunction, as I have previously discussed in phenomenological terms, will be extended to include the idea of sonic signification.

A broad discussion of music and meaning is well beyond the scope of this thesis. For my purposes, a particularly useful semiological perspective is the semiological tripartition proposed by Jean Molino and described by JeanJacques Nattiez in Music and Discourse: Towards a Semiology of Music (1990). To make his argument for a semiology of music explicit, Nattiez first describes the relationship between the producer of text, that is, written words, and the subsequent decoding of their meaning:

\footnotetext{
The meaning of a text - or, more precisely, the constellation of possible meanings - is not a producer's transmission of some message that can be decoded by a "receiver." Meaning, instead, is the constructive assignment of a web of interpretants to a particular form; i.e., meaning is constructed by that assignment. (1990, p. 11)
}

Nattiez makes the point that though a producer and receiver may both construct meaning, and that these two meanings may match, there is no guarantee that such a matching will occur:

With greater clarity than most scientific investigators, Pirandello has described this phenomenon: "the sad thing is that you will never know (and I can never tell you) how I interpret what you say to me. You have not spoken in Hebrew, of course not. You and I, we use the same language, the same words. But is it our fault, yours and mine, that the words we use are empty?...Empty. In saying them, you fill them with the meaning they have for you; I, in collecting them up, I fill them with the meaning I give them. We 
had believed that we understood one another; we have not understood one another at all" (Pirandello in Nattiez: 1990, p. 11)

Clearly there is a link here with phenomenology, in that both Nattiez and Husserl consider apprehension of an object, whether understood as sign or phenomenon, as a matter of intentionality and constituted by the receiver. Where a semiological appraisal of intention and reception is particularly useful, is in considering the phonograph as a particular class of intentional object (noema): one that embodies signification or traces.

In response to the mismatch between intention and reception as highlighted above by Pirandello, Molino developed the semiological tripartition; the 'three dimensions of the symbolic phenomenon:' the poietic dimension, the esthetic dimension, and the trace. The poietic dimension describes the process of creation, irrespective of the producer's intentions. The esthetic dimension describes the act of a 'receiver' ascribing meaning(s) to the symbolic form. The trace or neutral material as Molino described it, is the embodiment of the symbolic form in a material form accessible by the receiver's senses. Nattiez dedicates an entire chapter of his book to an analysis of the tripartition within electroacoustic composition. ${ }^{59}$ Though his analysis looks specifically at Schaeffer's l'objet sonore in relation to the semiological tripartition, aspects of his analysis are applicable to the works of soundscape composition.

Regarding acousmatic music (of which soundscape composition is a genre), Nattiez posits that 'the composer attempts to present a work as it is heard in the process of creation, without mediation of an intermediary' (1990, p. 91). Assuming Nattiez is correct, and in the case of the more dogmatic composers of musique concrète I believe him to be so, at this point we might conclude that little comparison can be made between musique concrète and soundscape composition, as musique concrète insists on the phonograph as the embodiment of the work through l'objet sonore, while soundscape composition attempts to illuminate source context, referencing the past, thus making a distinction

\footnotetext{
${ }^{59}$ Nattiez' chapter on electroacoustic music is entitled 'The Status of the Sound Object' (1990, p. 91-101)
} 
between the phonograph and the materials comprising the work at a temporal level: a listener is understood to hear both the past event and the new phonographic context. This indeed proves difficult when we consider the esthetic dimension of soundscape composition, as I will soon show, but still there is a striking similarity between the prescriptive methodologies of both musique concrète and soundscape composition on a poietic level.

Regarding acousmatic music, Nattiez reinstates distance between the poietic and the neutral levels by further defining the poietic dimension:

By "poietic" I understand describing the link among the composer's intentions, his creative procedures, his mental schemas, and the result of this collection of strategies; that is the components that go into the work's material embodiment. Poietic description thus also deals with a quite special form of hearing (Varèse called it "the interior ear"): what the composer hears while imagining the work's sonorous result, or while experimenting at the piano, or with tape. (1990, p. 92)

For Nattiez, the poietic dimension of acousmatic music is not simply the production of the work in a technical sense, but one that reaches into the intentionality and mental methodology of production. Understood in this way, the poietics of musique concrète and soundscape composition are quite similar; both utilise field recording, studio technology and 'mental schemas', while undoubtedly applying 'the interior ear'. It is the use of this ear that truly binds musique concrète and soundscape composition on a poietic level.

Though Schaeffer attributes 'reduced listening' to the role of the 'receiver' in his book Traite des objets musicaux (1966), that is, as a function of esthetic appreciation, Nattiez believes that this is in fact a poietic process:

I believe that Schaeffer's "concentrated hearing" is, contrary to his own implicit claim, essentially poietical in that it is in fact hearing as experienced by a composer, who hears sounds with extreme attentiveness before integrating them into a work. (1990, p. 95) 
Soundscape composition misplaces poietic processes in a very similar manner. While soundscape composition does not promote 'reduced listening', it does promote its own special kind of listening; one that might be termed 'soundscape listening; that is, listening to the sonic environment as defined by Schafer, influenced by the concerns of acoustic ecology. 'Soundscape listening', then, might well require a listener to hear sounds as representations of certain values, such as community spirit, ecology or preservation, or as representations of moral or social decay. The major point of difference is that 'soundscape listening', though also restrictive, requires a listener to engage with the soundscape as signification, where 'concentrated' or 'reduced' listening asks a listener to eliminate signification altogether and engage in a phenomenological form of listening. To confirm that soundscape composers indeed require a specific form of listening, we will recall Truax' second condition of soundscape composition:

2) The listener's knowledge of the environmental and psychological context of the soundscape material is invoked and encouraged to complete the network of meanings ascribed to the music. (2001, p. 240)

Accordingly, Truax determines that the materials of soundscape composition be understood by listeners as carrying 'environmental and psychological context'. I would argue that this is a case of Truax asking his audience to hear with his own poietic 'interior ear.' Like Schaeffer, Truax insists on a particular mode of listening, and does so through notes on record sleeves, programme notes at acousmatic concerts, and via his publications, including his widely read Acoustic Communication. In essence, soundscape listening, like reduced listening, is a form of listening that must be taught to listeners by composers.

In many respects, the poietic/esthetic confusion described above produces further confusion regarding the trace level. In order to understand this better, we must investigate what the trace level of a phonographic work might be. Nattiez argues that where the sound object is concerned 'the only proper sphere for esthetics' is the evaluation of the relationships between Schaeffer's sound 
objects. That is, the syntactical arrangements of materials within an acousmatic work (1990, p. 95). This may also be relevant to soundscape composition, and indeed any form of acousmatic composition. In this conception, understanding the divide between intention and reception is straightforward: though a composer may insist on a particular esthetic process via poietic listening prescriptions, the reality is that their message may not be received. No matter how a composer attempts to transmit meaning through their work, listeners will build their own meanings; fundamentally as a matter of intentionality and at the level of signification. Such a condition represents the semiological basis for reception theory. However, there is another element of the trace to consider that helps to clarify this disjunction further. It is an element I have referred to throughout this thesis as granting the phonograph its documentary power.

To reiterate, the documentary feature of the phonograph exists in every recording (as detailed in chapter one), be it an environmental recording or a recording of a synthesiser. There is a singular reality that is accessible by both the composer and listener: that the sounds of a recording were made in the past. Regardless of whether a listener readily perceives the past in a recording (musique concrète discourages this as I have shown), any listener who understands the technical functioning of a phonograph will know that such traces are there whether a composer promotes them or not. In semiological terms, it is a poietic feature of all works that exists as a trace level, accessible by the listener. Ontologically, it is a fundamental feature of all phonographs. Promoters of soundscape composition endorse this feature tirelessly: they want an audience to hear the past reality (despite the contradiction of schizophonia) in order to build their ecologically charged arguments. However, composers of soundscape works, including Truax and Westerkamp, believe that this trace affords access to much more information than it actually does. Much like Roland Barthes' concept of the photograph (the focus of chapter six), the phonograph simply states that something existed. It cannot embed the intentionality of the composer, or any supposed 'environmental or psychological context of the soundscape material' (Truax, 2001, p. 240). 
Pierre Schaffer's efforts to remove context from his sonic objects while prescribing reduced listening to his audience actively sought to deny this trace level an existence altogether. However, and as discussed in chapters one and two, such a trace level cannot be muted as it is a fundamental feature of the phonograph's ontology. Soundscape composition has a rather more complex relationship with this trace element. From Truax' writings, we might be led to believe that the trace, a soundscape's past reality, is recognised as carrying no inherent meaning due to the schizophonic condition, and that a composer, in presenting a second trace element (the composition of materials), reconstructs 'original' meaning. However, as I have argued, soundscape composers must believe that the materials of composition contain meaning, as without this capacity, no 'original' meaning could be 'reconstructed'.

As such, it is much more likely that soundscape composition adheres to something like the following logic: a soundscape has original meaning independent of a composer's intentionality. A composer, in experiencing this original meaning, records it with the aim of 'reintegrating' a listener with it. A phonograph, in attesting to the reality of a soundscape, also attests to the reality of this meaning. A composer then creates a composition, which further serves this meaning by making the inherent qualities of a soundscape explicit. A listener, in experiencing the reality of a soundscape, and the composition of their materials is 'reintegrated' with this meaning. This is the kind of process can be traced back to Westerkamp's assertion that 'materials inevitably speak with their own language' (2002), finding further support in the compositional methods detailed by Truax. If we examine this process critically, especially from phenomenological and semiological perspectives, we might conclude that soundscape composers endow the soundscape with deeper meaning, which can only be understood as the composer's intentional perspective on an environment and its value. Composers thus mistake the phonograph's trace level which asserts that 'something has existed', as something also laden with poietic intent. They also assume that through their composition, a listener will connect back to the soundscape's deeper meaning, which amounts to an assumed merging of the composer's intentionality with that of the listener. 
Needless to say, such a transaction between soundscape, composer and listener is a complex and doubtful prospect. Indeed, I will argue shortly that the only time soundscape composition can be deemed successful in its own terms, is when a listener is already well versed in the concerns of acoustic ecology, the techniques of the composer, and the materials of composition. This success cannot be read as a transmission of a composer's intentionality to a listener, but rather a pre-arranged meeting of intentionality.

Another concept relating to the intentionality of a composer has been proposed by Denis Smalley (1996). As stated above, two traces are observed in soundscape composition: that of the composition and that of the environment itself (a past reality). The nature of this environmental trace is murky given the complex structuring of meaning within soundscape. Smalley seems to advocate for the environmental trace that carries its own meaning, which, though problematic, is widely adhered to within soundscape composition, despite the continued influence of Schafer's schizophonia. Smalley proposes a transcontextual model for evaluating environmental sound composition:

\footnotetext{
In transcontexts the composer intends that the listener should be aware of the dual meanings of a source. The first meaning derives from the original, natural or cultural context of the event; the second meaning derives from the new, musical context of the composer. (Smalley 1996, p. 99)
}

This formula is useful from the perspective of a listener. As discussed in chapter three, when confronted with environmental sounds in an acousmatic environment, listeners will construct an intentional object in the act of perception, and it seems likely that such an object might be constituted of the phonograph, as rendered by a composer, as well as the events recorded. Soundscape composers however, do not truly intend that a listener be aware of the meaning derived 'from the original, natural or cultural context of the event', though they promote a similar notion. It is with the composer's 'interior ear' that these events are selected, and further composition attempts to make this bias explicit. In short, a listener is not asked to perceive the context of an event independently from a composition, but rather, through the act of 'soundscape 
listening' a listener is asked to perceive a composer's perspective of the event. In this way, where soundscape composition is concerned, the musical context of the composer is not distinct from the context of the original materials. Both are presented through the lens of acoustic ecology, as Westerkamp makes clear. The listener, however, may experience something closer to Smalley's transcontexts: they may (or may not) search for a composer's meaning while forming an independent bond with the source materials. Nattiez would describe this as esthetic analysis of the work, and phenomenology would have it as the construction of a noema.

I would like to briefly consider yet another trace element presented by the phonograph: that is, one born of the technology used in its production. This trace element is not of particular importance to this discussion of soundscape composition, insofar as composers often marginalise 'technology', seeing it as a vehicle for their compositional concerns as opposed to a feature of the trace level for esthetic engagement. My view of technology is that sound is embodied in it, as opposed to captured by it. Just as Ingold believes we launch 'into sound like a boat on the waves', so too do environments, launching into technology; transducing electrical current, distorting and phase shifting within transformers, magnetising tape heads, or resonating within the filters of equalisers. The sonic signatures of technology are wed to the environment from the moment the diaphragm of a composer's microphone moves. The sonic signatures of technology are profoundly important when considering the types of engagements a listener may have with the phonograph, and I will return to this topic in detail in chapter six. Before moving on, it is also important to note here that all of my compositions embrace the notion of sonic embodiment within audio technology, and that some pieces in particular explore the specific sonic signatures of the technology they employ. For example, 2CHPHNO-001 (2015) explores the sonic signatures of a Sony cassette recorder, while others focus on the spatial representation of ambisonic recording, the 'hi-fidelity' performance of certain microphones, or the use of postproduction effects in the rendering of a work. 


\section{The reception of soundscape composition}

As I have made clear through discussions of phenomenology and semiology, where environmental sound composition is concerned, there is an acute division between what a composer intends and what a listener receives. Where soundscape composition is concerned, this division may seem less apparent; given the methodology discussed above, we might assume that soundscape composers believe that such a divide is routinely bridged through successful composition. However, I would argue that contrary to the notion that composition itself can reintegrate a listener with an environment and the sociocultural context it contains, regardless of whether such context is believed to be embedded in the environment itself or ascribed by the composer, it is in fact the non-compositional, communicative activities of a composer that seek to engage a listener on this level. Further to this, I will argue that the successful conveyance of a composition's meaning from composer to listener is contingent upon a listener already having decided upon a composition's meaning (or at least its kind of meaning), independent of the composition itself, and that such connection should not be misconstrued as a transmission of message from composer to listener, but rather a meeting of the two entities in the predetermined, mutually agreed milieu of acoustic ecology and composition. In order to investigate this idea, I will turn to the writings of Jacques Rancière in his book The Emancipated Spectator (2009), as well as an earlier work The Ignorant Schoolmaster (1991), in order to evaluate the role of the receiver within an educational context. As we will see, Rancière's writings represent a very particular account of education, one in which the role of the student is elevated from that of a passive receiver of information to that of an active participant (if not the sole participant) in the accrual of knowledge. Such a perspective is valuable to this thesis, as it extends the notion of phenomenology (and reader response, a related idea discussed in chapter six) into the context of learning. By placing greater emphasis on the experience of the listener (or student in this context), the shortcomings of an educational outlook where environmental sound composition is concerned, become clearer. 


\section{The Ignorant Schoolmaster}

As Rancière recounts, Joseph Jacotot, a professor of French literature at the University of Louvain, developed ideas around the transmission of knowledge from a teacher to student in 1818. To begin, Rancière notes that prior to this time, Jacotot held a common view of this relationship:

Like all conscientious professors, he knew that teaching was not in the slightest about cramming students with knowledge and having them repeat it like parrots, but he knew equally well that students had to avoid the chance detours where minds still incapable of distinguishing the essential from the accessory, the principle from the consequence, get lost. In short, the essential act of the master was to explicate... (1991, p. 3)

As the word 'explicate' suggests, Jacotot believed that the role of the teacher was to reveal knowledge, while providing students with the tools to learn:

To teach was to transmit learning and form minds simultaneously, by leading those minds, according to an ordered progression, from the most simple to the most complex. (p. 3)

It might be argued that such a view of teaching is still widely held, as witnessed in the structure of many university degrees. However, Jacotot's 'intellectual adventure', as Rancière describes it, led him to conclude that such a teaching model did not reflect the reality of explication as he came to see it. Jacotot believed that the teacher as an explicator of knowledge was in fact a widely held misconception. The basis for this argument can be viewed in the way in which children learn language. Rancière notes that children learn language at a very young age without explicit instruction:

We speak to them and we speak around them. They hear and retain, imitate and repeat, make mistakes and correct themselves, succeed by chance and begin again methodically, and, at too young an age for explicators to begin instructing them, they are almost all - regardless of gender, social condition, and skin color - able to understand and speak the language of their parents. 
And only now does this child who learned to speak through his own intelligence and through instructors who did not explain language to him only now does his instruction, properly speaking, begin. Now everything happens as though he could no longer learn with the aid of the same intelligence he has used up until now, as though the autonomous relationship between apprenticeship and verification were, from this point on, alien to him. (p. 5-6)

From this Jacotot developed an understanding of explication that was contrary to how it was generally understood: instruction of students in a manner that promoted a false division of intelligence, that of a superior intelligence held by the teacher and of inferior intelligence held by the student, produced a stultifying effect. As Rancière has it, this insistence on a gulf between the teacher and the student 'is, in the first instance, the radical difference that ordered, progressive teaching teaches the pupil. The first thing it teaches her is her own inability. In its activity, it thereby constantly confirms its own presupposition: the inequality of intelligence. This endless confirmation is what Jacotot calls stultification' (Rancière, 2009, p. 9). At the most fundamental level, Jacotot believed in an equality of intelligence; a belief that all humans learn in the same way, as exemplified in the way that children learn to speak.

At this point, it might be asked in what way could Jacotot's ideas be applied to the works of soundscape composition? To argue that soundscape composers, in their didactic method of instruction, instate a similar gulf of intelligence between themselves and their listeners may seem crude. However, such an argument is not entirely unwarranted, for though an audience is, at least on the face of it, asked to explore acoustic ecology through the ephemeral and ethereal experience of acousmatic art on their own terms, i.e., without a composer's explication, in my view it is not the art through which instruction is truly made: it is through the words of the composer, spoken or printed, that their beliefs are made public, and through these words that listeners orientate themselves in relation to the composer, and ultimately, their compositions. Through these words, a composer attempts to explicate, the success of which I will question shortly with regard to another of Rancière's texts, The Emancipated Spectator 
(2009). I would first like to consider the importance of this contextual information (that which is not embedded in the phonographic work itself) or as Weale (2005) has it, the work's dramaturgy, to soundscape composition.

Though granting access to this dramaturgical information is not an explicit part of soundscape composition methodology as described by Truax - at no point does Truax insist that soundscape compositions must, for example, all have titles, and be accompanied by detailed programme notes - such information is typically included in the presentation of works and used as an orientating device. The notes to Westerkamp's Beneath the Forest Floor (1992), provide such an example:

Beneath the Forest Floor is attempting to provide a space in time for the experience of such peace. Better still, it hopes to encourage listeners to visit a place like the Carmanah, half of which has already been destroyed by clear-cut logging. Aside from experiencing its huge stillness a visit will also transmit a very real knowledge of what is lost if these forests disappear: not only the trees but also an inner space that they transmit to us: a sense of balance and focus, of new energy and life. The inner forest, the forest in us. ${ }^{60}$

I argue that this information is in fact the only way in which a composer can communicate with a listener about their concerns, as the composition alone, stripped of this dramaturgical information, does not hold the power to transmit the intentions of a composer to a listener.

As mentioned, Robert Weale refers to this kind of accessory information as belonging to a composition's dramaturgy, as describes in his $\mathrm{PhD}$ thesis, The Intention/Reception Project: Investigating the relationship between composer intention and listener response in Electroacoustic Compositions (2005). Weale's methodology is as follows:

The Intention/Reception project's methodology, the development of which is discussed in detail in this thesis, involves introducing RWE/A [real world electroacoustic] works that are unknown to the listening subjects, and then

\footnotetext{
${ }^{60}$ For full program notes for Beneath the Forest Floor (1992) See http://www.sfu.ca/ westerka/program_notes/forestfloor.html (assessed 21 October 2014)
} 
evaluating their listening experience. Through repeated listening and the introduction of the composers' articulation of intent (through a work's title, inspiration, elements that the composer intends to be communicated, eventually elements of the compositional process itself - in short, the 'dramaturgy' of the work) listening responses are monitored. The purpose here is to investigate to what extent familiarity contributes to access and appreciation and to what extent intention and reception are meeting in this very particular corpus of E/A art music. (2005, p. 2)

Weale positions his research within the context of similar studies, most notably Leigh Landy's. As Weale recounts, Landy's initial inquiry into the relationship between intention and reception was born of a desire to increase the audience for electroacoustic music. Landy's paper at the 1990 International Computer Music Conference, "Is more than three decades of computer music reaching the public it deserves?"(Landy, 1990, p. 369), concluded that 'E/A [electroacoustic] art music could and should access an audience outside of that which it generally accessed, and that based on this premise E/A art music research and artists' endeavour should devote a certain degree of its investigative energy towards addressing this issue' (2005, p. 21). Weale's study is located within this context, insofar as he attempted to determine the kinds of conditions under which a positive connection can be made between what a composer intends, and what a listener receives. Weale holds, as Landy does, that there could be a greater audience for electroacoustic music than it has, with the theory that 'by being offered something to hold on to (e.g., dramaturgic information) inexperienced listeners will be more able to access and appreciate a work and so have an engaging and enjoyable listening experience' (2005, p. 15).

The details of Weale's testing procedure, including the selection of composers and test subjects, the design of questionnaires for both composers and participants, and the structure of the test procedure can be summarised as follows. Participants were played three compositions three times, with response assessments completed between each presentation. At first, the participants were played each of the three compositions without any dramaturgical information, and asked for their responses. They were then given the titles of 
the works and played the three pieces again, and asked a new set of questions. They were then given access to a great deal more dramaturgical information, including specific details about the composer's intentionality, replayed the works, and asked a final set of questions. This procedure was designed to see how much dramaturgical information helped to assist in the appreciation of the three pieces. Through the course of this investigation, Weale found, unsurprisingly, that 'when offered pertinent aspects of a work's dramaturgy inexperienced listeners are able to use this information to assist their listening experience' (2).

Though each of the three pieces played included some form of environmental materials, none of the composers identified their works as soundscape compositions. However, Weale does point to Andra McCartney's PhD thesis, entitled Sounding Places with Hildegard Westerkamp (McCartney, 2000), which he notes 'is the one most related to [the Intention/Reception Project's] goals in terms of collecting data regarding composer intention and listener response and investigating the communicative relationship between the composer and the listener' (Weale, 2005, p. 74). Where soundscape works are concerned,

\footnotetext{
McCartney demonstrates the importance of including the communicative intentions and compositional strategies of the composer and analysis of the listening experience to a greater degree in E/A art music research with regards to understanding the communicative qualities of the soundscape work. "My method of analysis...makes evident the diverse conversations between composer and listeners, composer and researcher, musical work as composed and as heard." (McCartney in Weale, 2005, p. 75)
}

In her thesis, McCartney notes that the growing friendship between herself and Westerkamp further deepens her appreciation of Westerkamp's works. As such, Weale concludes that 'knowing intimate aspects of the composer's biography can shed light on compositional inspiration and communicative motivations and highlight the subtler aspects of a certain works' meaning content when undergoing poietic analysis' (2005, p. 77). This particular aspect, the biographical context of a composer, is vital to the educational goals of 
soundscape composition, as is the dissemination of the genre's cultural context more generally.

Returning to an educational perspective, we might conclude that for new listeners, soundscape composition in its wealth of dramaturgical information produces Jacotot's stultifying effect; that is, rather than raise awareness of acoustic ecology, the composition raises awareness of the listener's ignorance. This assertion however is a little crude, and I believe a more sophisticated analysis is required, which I will undertake shortly. For those who are already familiar with the dramaturgy of soundscape composition, the question must be asked: what is the educational value of soundscape composition? It could be argued that soundscape composition can make people aware of new works and indeed hitherto unknown environments, but how effective is soundscape composition in raising listener awareness of the concerns of acoustic ecology more generally? In order to explore this question, I will now turn to Rancière's The Emancipated Spectator (2009).

In the Emancipated Spectator Rancière picks up on ideas developed in The Ignorant Schoolmaster, chiefly, intellectual emancipation, and examines the role of the spectator in this context. Rancière dedicates a chapter of his book to what he terms 'the intolerable image', and though many of his other ideas find commonality with phonography more generally (see chapter six), it is this particular chapter that reveals the most where soundscape composition is concerned. To claim that soundscape composition shares features with images such as those of Martha Rosler's 'Bringing the War Home: House Beautiful' (1967-1972), requires some explanation. Rosler's images are what Rancière describes as intolerable. They consist of montaged photographs of luxurious New York apartment interiors, with gruesome images of the Vietnam War. Perhaps the most famous of these depicts a Vietnamese man standing in front of a large open-plan living room with a dead child in his arms: 
of those who enjoy this happiness to the intolerability of that reality and to their own complicity, in order to engage them in the struggle. $(2009$, p. 84$)$

It might seem inappropriate to compare the horrors of war with the concerns of acoustic ecology, however it is important to remember that it is the language of acoustic ecologists and soundscape composers themselves that give this comparison some credence. Schafer reminds us that with schizophonia, 'sounds are torn from their natural sockets' (Schafer, 1977, p. 90), describing the act of recording in acutely violent terms. Likewise, in Kits Beach Soundwalk (1989) Westerkamp likens the gentle city hum to a monster: 'play with the monster, then I can face the monster,' she recites as the city reasserts itself at the end of her piece. Though in more recent years soundscape composers have been actively trying to build a more positive, celebratory notion of how the soundscape should be promoted, a tenacious idea persists: in order to raise awareness surrounding acoustic ecology, particularly noise abatement, noise, be it the city hum or a product of schizophonia, needs to be portrayed in a negative light, as seen in Kits Beach Soundwalk, in order to make the point explicit. ${ }^{61}$ Soundscape composition of this order designates noise as 'intolerable', and uses techniques that bear comparison to Rosler's in order to make the point.

Using Kits Beach Soundwalk as an example, Westerkamp accentuates the intolerable city hum by accentuating it through the loudspeakers; the very medium that facilitates schizophonia, which, perhaps inadvertently, draws attention to our complicity as consumers of audio technology in the increase of the global noise-floor. Just as Westerkamp 'plays with the monster' between the loudspeakers, so does Rosler, playing with her monsters here on the stairwell or there outside the window of the luxurious apartment. Rancière notes:

\footnotetext{
The view of the dead child in the beautiful apartment, with its bright walls and vast proportions, is certainly difficult to tolerate. But there is no particular reason why it should make those who see it conscious of the reality of imperialism and desirous of opposing it... for the image to produce
}

\footnotetext{
${ }^{61}$ In 'Acoustic Ecology of Great Places' (2014), John Drever asserts that acoustic ecology is developing a more positive ear towards the soundscape. http://www.academyofurbanism.org.uk/acoustic-ecology-of-great-places/ Accessed 27/11/2014
} 
its political effect, the spectator must already be convinced that what it shows is American imperialism, not the madness of human beings in general. She must also be convinced that she is herself guilty of sharing in the prosperity rooted in imperialist exploitation of the world. And she must further feel guilty about being there and doing nothing; about viewing these images of pain and death, rather than struggling against the powers responsible for it. In short, she must already feel guilty about viewing the image that is to create the feeling of guilt. $(2009$, p. 85$)$

In much the same way, soundscape composers attempt to raise awareness about the increasing global noise-floor, the endangerment of certain soundscapes and the struggle to preserve them. However, their works fail to raise awareness, to inflict a sense of guilt or to shock a listener into action, as in order to deduce a political message from soundscape composition, a listener must already be convinced of the work's politics. By way of example, in order to understand Westerkamp's city hum as monstrous, a listener must already believe the city hum to be a negative environmental feature, and be closed to its aesthetic interest. In addition, listeners must also be aware of their complicity in the production of noise. In short, soundscape composers do not achieve their desire to raise awareness of acoustic ecology through composition, as those listeners who successfully generate the intended meaning from the work, are already orientated within acoustic ecology.

This observation is related to my belief that the sonic environment does not carry any inherent meaning. Much like Fisher (1998), I understand the sonic environment as a phenomenon that lacks the appropriate features to afford a collective aesthetic response to it, ${ }^{62}$ and as such, an environment's 'deeper meaning' can only be understood as what an individual composer has it to mean. I also contend that soundscape composers cannot impart their intentionality through composition alone. Dramaturgical information must be used in order to orientate the listener to the composer's 'interior ear', and without this information, a listener is left with no way of knowing what a composition may

\footnotetext{
${ }^{62}$ While I believe some societies, such as the Kaluli of Papua New Guinea (Feld ,1994), may exhibit a heightened and perhaps more communal appreciation of the sonic environment, I do not believe that sonic environments can carry meaning independent of those who hear them.
} 
mean, as regardless of what composers believe, neither environmental materials nor their arrangement and manipulation have the ability to convey their intentionality, let alone any intentionality they may ascribe to an environment. As such, soundscape composition alone lacks the ability to educate listeners about the concerns of acoustic ecology. Extensive dramaturgical information is employed to help orientate listeners, but even this does not guarantee that listeners will respond to works in the desired manner. For example, I am empathetic to the concerns of acoustic ecology, though I, like Cusack and Kubisch, do not hear the city in the same negative way that Westerkamp does; I am not shocked by it.

\section{Summary}

Where the production of environmental sound composition is concerned a number of interesting perspectives emerge. As discussed, phonographers attempt to reveal the world to their listeners through the methods of temporal and spatial framing. There is no underlying dogma associated with phonography: within the genre we might include the works of Lopez with his focus on spatio- and spectromorphology, those of Ferrari, with his focus on narrative, and also Winderen, with her desire to impart a sense of place. At the far end of this spectrum, where a composer attempts to communicate ecological concerns directly to a listener through composition, we find soundscape composition. Soundscape composers, exemplified in the writings and works of artists such as Truax and Westerkamp, manifest confused relationships with the concepts promoted by the genre. The concept of schizophonia, while supporting the phonograph's abstractive ontology, does so while muting its documentary power; the two are considered mutually exclusive. Yet it is the documentary power of the phonograph that is exploited in order to support the genre's educational aspirations. Indeed the notion of a soundscape's 'deeper meaning' and its transmission through the phonograph to a listener relies on this feature. Intentionality is also an area of confusion within soundscape composition. Though not all composers endow the soundscape with intentionality as Westerkamp does, many composers assume that their own intentional 
perspective of the environment will be transferred to a listener through the recording process and made explicit through composition; that their intentionality is embedded in the phonograph at the trace level.

Though a phenomenological, semiological and educational perspective might reveal some of the shortcomings in poietic and esthesic understandings of an environmental sound composition, it is largely from a poietic perspective that we understand the failings of intention and reception. An esthesic analysis of environmental sound composition remains rare and elusive. In other words, though environmental sound composers, soundscape composers especially, are a great pains to describe the what and why of their works, the how of their reception remains largely untouched, and it is this inability to adequately account for phonographic reception that undoes much of the creative energy invested in environmental sound composition. Though The Intention Reception Project looks at audience reception of environmental sound works, it does so from the hypothesis that an increase in dramaturgy aids in the aligning of a composer's intentionality with that of the listener's, and that such an alignment results in a richer experience for the listener. This bias is clearly observed in the project's test methodology. The Intention Reception Project thereby reinforces the same notion found in soundscape composition: that compositions are successful if the composer's intentionality is communicated through the work and met by the intentionality of the listener (albeit through the work's dramaturgy). I argue, as Rancière might, that the provision of such dramaturgy does not effectively educate the audience, nor does a composition without dramaturgy. By orientating the listener to their concerns before listening, soundscape composers might increase awareness of their poietic intent, but from an educational perspective, the listener must already have decided where they stand with regard to acoustic ecology in order to "hear the message". As argued throughout chapters three and four, a transaction whereby a composer educates a listener is flawed, given the complexity and specificity of composer intentionality and the inability for the phonograph to embody this intentionality. More importantly, the model fails to acknowledge the complex and specific nature of listener intentionality. Though some listeners may wish to 
seek out a composer's poietic intent, this may not be a significant aspect of a listener's total experience of the work. As I will argue in chapter six, the composer's intentionality may in fact be the least engaging aspect of a listener's experience, or in some cases, it may even hinder a positive response. 


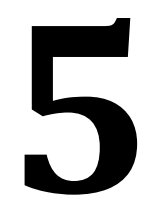

\section{Aural and Visual Experience: Phonography, Film and Photography}

In order to gain a better understanding of the way in which listeners engage with the phonograph, especially where environmental sound composition is concerned, I have undertaken research into other mediatised experiences, drawing the conclusion that photography provides the best points of comparison. At face value, a comparison between phonography and photography may seem unlikely to yield meaningful results, due chiefly to the temporal difference between the two: the camera's freezing of an instant versus the speaker's replay of a scene. Film, as a medium with a similar temporal dimension to that of the phonograph may at first appear to provide a more fruitful comparison. As I will discuss however, the multi-modality of film, and its 'audiovisual contract' as Michel Chion (1994) puts it, complicates the comparison on a number of levels. Silent film might therefore seem to provide the best comparison: it has a single modality and a comparable temporality. Perhaps even 3-D silent film, with enhanced spatiality would provide the best comparison. However, the moving image produces quite a different mediatised experience to that of recorded sound. Photography, as I will make clear, provides phonography with the most useful comparison, despite the obvious difference in their temporal form. The comparison I will make draws from Roland Barthes' hermeneutic analysis of the photograph in Camera Lucida (1981), augmented by my own analysis of the phonograph, as developed in this thesis. A detailed account of the relationship between photography and phonography follows in 
chapter six. This chapter looks at why other mediatised experiences are less appropriate, though in the process, a great deal is revealed about the specificity of the phonograph.

Before beginning an initial discussion of photography and the phonograph, it should be noted that a comparison of the phonograph with film and photography necessarily includes a comparison of aural experience and visual experience. In The Audible Past (2003), Jonathan Sterne asserts that such comparisons can contribute to what he describes as the 'audiovisual litany', a phrase he uses to denote a perspective of sound and vision that reinstates 'the long standing spirit/letter distinction in Christian spiritualism' (p.16). While Sterne questions 'the purportedly special capacities of each sense as the starting point for historical analysis' (p. 15-16), that is not to say that such a comparison is without value. I will argue that aural and visual perceptions are undoubtedly different (without wishing to promote Christian spiritualism or any such theology), and that in exploring their unique characteristics much can be learned about the experience of mediatised events.

\section{Photography and the nominalist 'work' conception}

A comparison between phonography and photography is relatively common. In his book Absolute Music, Mechanical Reproduction (2010), Arved Ashby connects the similarity between the photograph and audio recording to the nominalist conception of the work as presented by Lydia Goehr, discussed in chapter one. Within the nominalist conception, the temporal dimension of performance, playback and musical appreciation is not considered a part of the work itself, but rather a product of the work's actualisation. The work itself is understood as a unified, independent structure, accessed by the composer, who renders approximations of it through scores and performances, thus granting limited access to the work to listeners. Ashby provides many examples of individuals, conductors especially, referring to recordings as providing such limited access and having a moment-in-time, snapshot quality. The most explicit example of this is articulated by Boulez: 
"A recording, you know, is a picture," Boulez points out. "You take a picture of a work at a certain time." (Boulez in Ashby, 2010, p. 199)

Ashby notes, 'clearly, in Boulez's view a classical composition is something to be "taken" or "snapped" rather than "shot", as one does a movie' (p. 199). Though Ashby believes Boulez to hold a clear view on this matter, it should be noted that Boulez understands picture taking as analogous to audio recording, which makes his view less precise than Ashby suggests. The discussion that follows questions the value of this analogy.

To begin with, there can be little doubt that through this analogy, Boulez seeks to promote an understanding of the work as nominalist; an object that could be "snapped" if it could be seen. While this analogy is clearly figurative insofar as the abstract, non-sounding work cannot be "snapped", "shot" or mediatised in any way, it does have the effect of diminishing the value of the phonograph by marginalising its temporal realisation and attributes, which further diminishes the phonograph to a documentary role, in service to the (inaccessible) work proper. Boulez' comparison between the photograph and the phonograph may even be seen to strip the work of its temporal attributes altogether, promoting the work as a temporally independent object. In my view this particular analogy is without much value. It does, in fact, unnecessarily confuse the relationship between the phonograph and the nominalist work. If indeed the purpose of the analogy were to instate a clear divide between the all-important work and its subservient document, or, as Husserl would have it, the noema and its adumbration, ${ }^{63}$ it would seem more prudent to promote the temporal dimension of mediatisation by likening a recording to film, thus making a clear distinction between the static abstract work and its spatio-temporal manifestation.

By suggesting, even in passing, that the phonograph has the ability to capture the work in an instant, Boulez equates the abstract work with its performance. In

\footnotetext{
${ }^{63}$ Husserl's adumbrations, in phenomenological terms, are understood as limited aspects of the noema, inferred through hyletic data. In this way, the phonograph might be understood as an adumbration of the nominalist work. See chapter four for my analysis of the adumbration within the context of sound, and Philipse, (1995, p. 239-323) for a broader perspective.
} 
doing so, we might conclude that Boulez holds a nominalist conception of the work that is Aristotelian in nature: a view in which the work, though abstract, is dependent upon performance in order to be actualised. However, even if this were the case Boulez' picture would position the phonograph as a picture of the work and not the work itself. In order for Boulez to believe a recording is in any way a picture of a work, he would need to hold what Goehr describes as the Analytical view of the work, in which the work has no abstract existence independent of a performance or recording. In this instance, however, Boulez wouldn't be able to ascribe the work a unified and static existence capable of being captured in an instant; rather it would hold the same temporal qualities as that of the phonograph, a format that would be considered excellent for capturing the work conceived of in this analytic form. Though I will not unravel Boulez's particular conception of musical works any further, his insistence that a 'recording... is a picture' suggests a nominalist view of instrumental composition in some capacity.

Another genre where the photographic analogy might be applied, on the basis that the musical work is nominalist, is soundscape composition. As I have pointed out in chapter four, promoters of acoustic ecology can be seen to inadvertently solidify the temporal and spatial dimensions of their soundscape, effectively objectifying the environment in much the same way Boulez, Stravinsky and others objectify the musical work, yet soundscape composition explores the temporal dimension through composition in order to create narrativity and ultimately, meaning. As such, while soundscape composition may promote the soundscape as a fixed object defined by non-temporal qualities, the practice does not seek to deprive it of its temporal dimensions. With this in mind, we might conclude that the composers of soundscape works, while idealising and objectifying the soundscape never seek to separate it from its sounding.

In my view, to liken phonography to photography on the basis that they share the same moment-in-time temporality is deeply problematic. More importantly, to deny a recording this temporal dimension effectively excludes any discussion as to how a listener may perceive temporality in a recording, and what the 
implications of such perception might have on their analysis of the phonograph and/or work. The temporal dimensions of the phonograph are so central to discussions of its ontology that I assert any suggestion that a phonograph lacks temporality, either literally or figuratively, can be dismissed. Nonetheless, despite the temporal difference between the phonograph and the photograph, the apparent incongruence between the two gives way to a much greater affinity than might first be imagined. First however, it is vital to examine the phonograph in relation to film, a format that shares a similar temporality.

\section{Film and Phonography}

As suggested above, film and phonography might appear to have much in common insofar as they a share similar temporal ontology. However, given that film typically involves a marriage between moving image and audio recordings, the multi-modality of film makes this comparison somewhat difficult. Before arguing that film with sound is inappropriate for comparison with the phonograph, it is important to explore what each element has to offer the other within what Michel Chion describes as 'the audiovisual contract' (Chion, 1994).

Above all, it should not be understated just how complex the audiovisual contract is, and therefore how difficult an analysis of sound with sound and vision would be. To begin with, sound is often considered to play a supporting, subordinate role within the audiovisual contract. As a matter of attentional focus, sound is considered of secondary importance. Furthermore, the marriage of sound and vision in film presents a spectator with a unique phenomenon, which includes complex relationships involving perception of the past and present, movement and temporal progression and through visual and audial interactions. In AudioVision: Sound on Screen (1994), Michel Chion explores the inter-relatedness of the aural and visual senses in film, with a core feature being how each 'adds value' to the other. He writes:

Visual and auditory perception are of much more disparate natures than one might think. The reason we are only dimly aware of this is that these 
two perceptions mutually influence each other in the audiovisual contract, lending each other their respective properties by contamination and projection. (1994, p. 9)

Chion illuminates some interesting features of aural and visual perception in the course of his discussion. Concerning motion and stasis, Chion notes that 'sound, contrary to sight, presupposes movement from the outset' (1994, p. 9). Though sound may offer stasis in the form of steady-state sound wave propagation, such as the sound of a generator or, as Chion offers, a telephone's dial tone, 'it is rare not to hear at least some trace of irregularity and motion' (p. 9). This first point shows how sound is contingent upon motion of some description, while film may be static, moving, or a mixture, and with various states in between. Chion also intimates that humans can detect very subtle changes in sound, a point he expands on by stating that there is a disparity between the rate of perception of the visual and aural senses, claiming aural perception is much faster at processing information than visual perception:

Sound perception and visual perception have their own average pace by their very nature; basically, the ear analyzes, processes, and synthesizes faster than the eye. (1994, p. 10)

Arthur R. Jensen's research, in Clocking the Mind: Mental Chronometry and Individual Differences (2006), supports Chion's assertion, noting that 'the fastest visual and auditory SRTs [Simple Reaction Times] are about 180 and 140ms respectively' (p. 47). ${ }^{64}$ Further to this, Chion also posits that

\begin{abstract}
The eye perceives more slowly because it has more to do all at once; it must explore in space as well as follow along in time. The ear isolates a detail of its auditory field and it follows this point or line in time... so, overall, in a first contact with an audiovisual message, the eye is more spatially adept, and the ear more temporally adept. (1994, p. 11)
\end{abstract}

This particular notion appears as the tenth point on Sterne's list of audiovisual litanies: 'hearing is a primarily temporal sense, vision is a primarily spatial

\footnotetext{
${ }^{64}$ Jensen also notes that the reaction time to touch is about $150 \mathrm{~ms}$, and that reaction times to taste and smell are hard to measure, but they are 'relatively slow and imprecise' by comparison (2006, p. 47). Given the 40ms difference in reaction time between aural and visual perception, Jensen concludes that 'transduction of a visual stimulus takes longer, presumably because it involves a chemical process in the retina, whereas audition involves a quicker, mechanical action' (p. 47).
} 
sense' (2003, p. 15). Though designated as a litany, it should be noted that Sterne does little to discredit the validity of such an observation beyond questioning its social and historical origins. Though it might be argued that hearing and vision are not primarily temporal and spatial senses respectively; that is, each sense contributes to temporal and spatial awareness to some degree, the contribution of aural perception to temporal awareness should not be understated.

Chion's assertion finds support in the writing of acoustician Jian Kang, who notes that 'sound provides dynamism and a sense of reality, helping people to get the sense of the progression of time' (Kang, 2007, p. 48). This observation reveals a direct link between the perception of temporal progression and a 'sense of reality', a point that Chion pursues further in his analysis of temporality. Highlighting the temporal adeptness of the ear is of particular value to the discussion of the phonograph's ontology. Chion describes the 'three aspects of temporalisation' in the audiovisual contract: temporal animation, temporal linearisation, and temporal vectorisation. Of temporal animation, Chion states that 'to varying degrees, sound renders the perception of time in the image as exact, detailed, immediate, concrete...' (1994, p. 13). Where a largely static image is concerned, sound may provide an image with a sense of time passing which may be otherwise difficult to detect, such as an interior shot of a room filled with inanimate objects. Where an image is animated, perhaps with a mixture of moving and static objects, sound enforces a sense of real-time progression, adding micro-detail and spatial information. In other words, it is the aural dimension that renders temporality in film exact.

Temporal linearisation describes the way in which sound provides images with sequential linearity:

When a sequence of images does not necessarily show temporal succession in the actions it depicts - that is, when we can read them equally as simultaneous or successive - the addition of realistic, diegetic sound imposes on the sequence a sense of real time, like normal everyday experience, and above all, a sense of time that is linear and sequential. (1994, p. 17-18) 
This feature shows just how compelling aural perception is regarding a listener's perspective of time. An image may jump from one scene to another, to the extent that, if viewed without sound, they may be identified as unrelated. Sound, however, ties potentially unrelated images together. We cannot say that the reverse is true: a continuous camera shot will not homogenise disparate audio into a temporally believable sequence. For this reason, it is sound which has the truth-telling capacity often attributed to sight ("seeing is believing"); as Chion states above, aurality is the dominant sensorial field in the perception of realtime, linear progression.

Temporal vectorisation is another feature of the audiovisual contract that emphasises the role of aurality in temporal awareness in film. Chion provides an excellent example:

Imagine a peaceful shot in a film set in the tropics, where a woman is ensconced in a rocking chair on a veranda, dozing, her chest rising and falling regularly. The breeze stirs the curtains and the bamboo wind chimes that hang by the doorway. The leaves of the banana trees flutter in the wind. We could take this poetic shot and easily project it from the last frame to the first, and this would change essentially nothing, it would all look just as natural. (1994, p. 18-19)

Where temporal progress is visually fungible it is not so sonically. Sound, in this instance provides us with the only indication of the 'correct' progression of time. If we were to play the synchronous audio of this hypothetical scene backwards, last sample to first, we would encounter the unmistakable signatures of reversed audio, with sound's decay profiles leading attack transients and spatial information, such as a sound's reflection, preceding their stimulus. Chion concedes that in many instances the same signatures may be perceived in reversed moving images, pointing to 'inevitable gags' such as the reversal of exploding objects. However, he notes:

But much more frequently in movies, images of a character who speaks, smiles, plays the piano, or whatever are reversible; they are not marked with a sense of past and future. Sound, on the other hand, quite often consists of a marking off of small phenomena oriented in time. Isn't piano music, for example, composed of thousands of little indices of vectorized real time, since each note begins to 
die as soon as it is born? (1994, p. 20)

Chion's analysis of audiovisual temporality leads us to two important conclusions regarding the nature of sound. The first of which is that sound enforces a sense of the present with some authority. This is aided by sound's vectorisation of time, whereby the attack and decay of Chion's piano for example, indexes time, providing a sense of the present and the passing of time. As Chion concludes, when real-world experiences are mediatised, this feature of auditory perception remains intact and is exploited in film, in order to provide the film with a sense of 'the present' that mediatised visuality no longer carries with the same conviction. This is not to say that film cannot vectorise and therefore index time; indeed vectorisation is contingent upon the perception of movement, movement that can also be detected in the moving image. In Cinema 1: the Movement Image (1986), Deleuze argues that

cinema does not give us an image to which movement is added, it immediately gives us a movement image. It does give us a section, but a section which is mobile, not an immobile section + abstract movement. (1986, p. 2)

Regardless of the exact nature of movement in moving images, sound is, without question, contingent on movement, is perceived as such, and thus plays the dominant role. Additionally, the correct vectorisation of time is consistently granted a listener through aural perception, whereas sight is much less consistent, as Chion's hypothetical scene attests; visual movement can present a level of ambiguity that aural movement does not. Furthermore, the presence, immediacy and temporal believability of sound is maintained through mediatisation not simply as a result of the greater speed of aural perception, though this undoubtedly contributes to the phenomenon, but also as a result of a wedding of the source of the sound and the new acoustic environment within which it sounds. In other words, a greater sense of reality and immediacy is afforded mediatised sound on the basis that it engages with the new space in a way that visual media does not. I will return to this point with more detail later in this chapter. 
The second point that Chion's analysis reveals, is born of the first; that is, the physically determined progression from one sound to another, with all the appropriate ordering of aural information, such as the attack and decay transients of sounds, provides a listener with a detailed, believable account of real-time passing. As such, when listening to a recording of an event, a listener is made aware of a past reality. Though a listener may not be able to identify the source of a sound, so long as it obeys the laws of physics, an event will most likely be perceived as actually having happened. Composers often exploit this perception in order to make temporally disparate recordings seem congruent, as witnessed in the pseudo-documents of Glen Gould, recounted in chapter one. It is the vectorisation of time that makes such recordings believable. When we consider the shot of Chion's fictional woman, ensconced in her rocking chair moving as naturally from last frame to first as from first frame to last, we must conclude that the laws of physics are not as easy to identify where mediatised moving images are concerned, hence the value sound adds to the audiovisual contract. With these two points in mind, we can conclude that recorded sound renders the past in the present with a degree of certainty that eludes visual media, and it does this through the exceptional ability of aural perception to apprehend and process hyletic data, and its ability to account for the passing of time. I will now turn in greater detail to the way in which temporality features in our perception of 'the present'.

\section{Experience of 'the present' in aural perception}

To begin with, it should be noted that many theories of listening promote different types of attentiveness, which in turn suggests different perceptions of the present. Without detailing a myriad of possible listening modes as Hollerweger does in his PhD thesis The Revolution is Hear! (2011), or describing the analytical processes of the brain as Bregman does in Auditory Scene Analysis: the Perceptual Organization of Sound (1990), I will simply observe that a variety of conscious and subconscious listening states exist between what might be considered attentive and inattentive listening. At the most attentive end of the spectrum, Schaeffer's 'reduced listening' attunes our perception to the present, in a way that Truax' 'listening-in-search' does also (Truax, 2001: 21). Though 
Schaeffer asks a listener to strip away contextual information while Truax asks us to embrace the contextual, reduced listening and listening-in-search both envisage a listener as one whose attentional focus is attuned to the onset of aural information. At the least attentive end of the spectrum we might find one who is asleep; though their ears are functioning, their brain is resting, and thus not focused to the present. However, when we focus through aural perception on sound, be it as an act of listening to something (music, nature etc.,) or as a result of impaired vision either through acousmatic music (Schaeffer, 1966) or blindness (Copeland, 2000; Hull, 2001), we become acutely aware of the present and the succession of passing time.

Don Ihde, who considers aural focus in relation to temporality, makes the most useful analysis of attentive listening. In analysing Husserl's concept of 'inner time consciousness', Ihde notes that:

\begin{abstract}
A phenomenology of experienced temporality soon comes on the notion of a temporal span or duration of sounding that is experienced in listening. I do not hear one instant followed by another; I hear an enduring gestalt within which the modulations of the melody, the noises present themselves. The instant as an atom of time is an abstraction which is related to the illusion of a thing in itself. In terms of a perceptual field we have noted that a thing always occurs as situated within a larger unity of a field; so temporally the use of instant here is perceived to occur only within the larger duration of a temporal span, a living present. (2007, p. 89)
\end{abstract}

In suggesting that we encounter a temporal span when listening suggests that listening and temporal awareness are intrinsically related. Ihde makes this point clear:

When I listen to auditory events, there seems to be no way in which I can escape the sense of a "coming into being" and a "passing from being" in the modulated motions of sound. Here temporality is not a matter of "subjectivity" but a matter of the way the phenomenon presents itself (p. 94).

Husserl refers to this "coming into being" and "passing from being" as protentions and retentions respectively, and that the time between the onset of the 
protentions and the conclusion of retentions represents the field within which our aural perception operates. Ihde uses the term temporal focus to describe our attentional intentionality (p. 90). In Ihde's view, and as the term suggests, this temporal focus has the ability to traverse the temporal span, and with an attentional depth, understood as an ability to bring certain auditory data to the foreground of our intentionality, while relegating others to the background. This attentional depth may have a broad or narrow focus depending on the intentionality of the listener. Of this attentional depth, Ihde suggests that a broader focus may be employed while listening to relaxing instrumental music. In this instance, an individual's attention maybe less concerned with a foreground and background, and may be termed 'panoramic' (p. 90). The ability to 'pull' aural data to the foreground is often acknowledged by various researchers in different fields. Within the context of information theory, Truax notes our ability to tuneout the repetitive, redundant sounds found in urban environments in order to focus pertinent information (2001, p. 19), while Bregman recounts his own version of Colin Cherry's 'cocktail party effect', in which he displays an ability to determine the timbre of a friend's voice despite the competing frequencies of the clinking glassware at the party $(1990$, p. 2). Ihde's analysis of this narrow perceptual focus includes a compelling account of the way in which it operates within the temporal span:

\footnotetext{
If I am to be the subject of a psychological experiment in which a click is to be the signal of some action, I listen intently for that short and barely enduring sound. My protending expectation "searches" the futural "edge" of the temporal span in order to be prepared for the onset of the click. I have "pushed" all other auditory-temporal factors into the background and I listen only for the click. (2007, p. 93)
}

While it might seem likely that attentional intentionality will always focus our perception to this futural edge as we search out specific data, Ihde presents another hypothetical experiment that displays the way in which we have the ability to shift focus away from this edge:

I am now to listen to a tone to identify its position in the musical scale. Again I listen intently with the same selectivity for the tone. This time at its presence I do not attend specifically to its instantaneous source-point, but 
pay special attention to its tonal quality, which appears even more strongly in its "running off" reverberation, and I identify it as middle C. (2007, p. 93)

Here, Ihde's focus is aimed at the "running off" of the temporal span, though he listens just as intently as he did for the click in the previous example. He also notes that while temporal focus is often aimed at the futural edge (protentions) or the "running off" (retentions) of the temporal span, a broader focus may be employed. Again, Ihde uses the example of musical listening to illustrate a broader temporal focus, this time of the temporal dimension, by suggesting that 'one usually allows the full richness of the musical presence to occur in what is here a broad or open focus with the onset of each note enriched by the depth of those that have just preceded it "equally" present.' (2007, p. 94)

Ihde's appraisal of the temporal span and temporal focus tells us a great deal about the perception of time in aural experience, augmenting Chion's account of the realness of temporal progression with a phenomenological account of how temporality is manifested in aural perception. He also notes how moving phenomena specifically (spatial movement in particular), including sound, hold a privileged, direct relationship with the perception of time. As mentioned, movement in visual perception can also index time, as Deleuze (1986) would argue, though when we recall Chion's observation that 'sound, contrary to sight, presupposes movement from the outset' (1994, p. 9), we might conclude that aural perception is the more privileged of the two senses in this regard. Ihde supports this notion with the following analogy:

If I look at the calendar on the wall, it stands out as motionless and mute, and in relation to it I detect only a massive newness. Its appearance neither dramatically comes into presence nor passes from it in its motionless state. If I want to take note of its "temporality" I must already make a reflective turn to noetic phenomena: it is my consciousness that is aware of the passing of time before this object. However, if the object is moving, my son's baseball suddenly looms before me, and I must either catch it or avoid it before being hit - in the duration of the event of the ball coming toward me the moving ball allows a shift toward the noematic appearance of successive time. (2007, p .94)

With this in mind, we can conclude, as Ihde does, that aural awareness is 
predisposed to the perception of the present, understood not as an instant, but as a temporal span between the protending edge and the horizon of retention, in a way the visual awareness is not. To focus on the passing of time in visual perception requires a conscious shift that aural perception does not require. Though movement within the visual field seems to hasten this process, as I will discuss later in this chapter, the ability to engage with the temporal span through visual perception is of an entirely different order to that of aural perception.

Applying this phenomenological approach to mediatised events, we can see the perception of the present remains largely unaffected by recording, as the passing of time is perceived in the consciousness of the individual. As such the passing of time can be perceived in a situated experience of a day at the beach, or a recording of one, such as Ferrari's Presque Rien No. 1, made close to half a century ago. Though, as I will discuss in chapter six, we may be able to detect and contemplate the past in such a recording, we do so presently; that is, with an acute awareness of the present: a presence of the present. Unlike photography, our present cognition is not drawn to the past. This is an important observation and I will expand on it shortly within the discussion of protensity.

Returning for now to Chion's audiovisual contract we can conclude that a comparison between film and phonography is not of significant value here, as the multi-modality of film does not readily allow for singular points of comparison with the mono-modality of the phonograph. However, Chion's analysis of the audiovisual contract provides an excellent account of how the perception of sound operates, how it differs to visual perception, and especially the role sound plays in our perception of temporality, presence and reality. Ihde's phenomenological approach enhances Chion's observations. With these aspects of the phonograph's temporality to hand, it can be seen why a comparison between audio recordings and silent film isn't particularly beneficial, insofar as their temporal dimensions are not as compatible as they might first appear. In short, silent film lacks the kind of immediacy and 'realness' of phonography, especially in instances where visual editing is apparent, without the soundtrack to provide linearity and a sense of real-time progression. Perhaps then a single shot, fixed position silent film reveals a greater similarity with the phonograph, 
but further investigation into this comparison will reveal the role of visuality in our perception of the real as a confounding factor in this comparison.

\title{
Silent Film
}

Single shot silent films were common in the late nineteenth century, and they were indeed noted at the time for their extraordinary realism. Famously, the first screening of Auguste and Louis Lumière's short film L'arrivée d'un train en gare de La Ciotat (1895) in Paris is said to have caused the audience to retreat in panic to the back of the cinema as the train approached the camera, such was the realism of the film. However, Martin Loiperdinger dismisses this story as fiction with some conviction. Loiperdinger suggests that audience members attending this film, though encountering what must have been a new and fantastic experience, would not have had difficulty distinguishing fact from fiction. He suggests that 'the reputed cries of fear among the audience can hardly be attributed to a confusion of reality and projected image' (2004, p. 104). He recounts the experience of Tom Gunning, who likens his experience of L'arrivée d'un train en gare de La Ciotat to that of taking rides at a fun park:

\begin{abstract}
The on-rushing train did not simply produce the negative experience of fear but the particularly modern entertainment form of the thrill, embodied elsewhere in the recently appearing attractions of the amusement parks (such as the roller coaster), which combined sensations of acceleration and falling with a security guaranteed by modern industrial technology. (Gunning in Loiperdinger, 2004, p. 104)
\end{abstract}

Gunning's assessment might be more broadly contextualised within what Coleridge famously described as a 'willing suspension of disbelief', in which an audience puts aside their understanding of fiction in order to enjoy the content as if it were real, as a matter of 'poetic faith'. 65 The nature of this suspension has been the subject of some debate which I cannot enter into here, but applied to L'arrivée d'un train en gare de La Ciotat, we might conclude without much difficulty that audiences, from the safety of their seats, enjoyed the thrill of seeing a train approaching, giving themselves over to the fantasy that it might breach the

\footnotetext{
${ }^{65}$ Samuel Taylor Coleridge first used the term in his Biographia Literaria (1817), Chapter XIV, regarding his works of poetry, and how he believed his 'supernatural' content should be understood by his readers.
} 
fourth wall of the cinema screen, without actually fearing for their lives. As such, though 2D moving images project a sense of 3D space behind the screen, they do not project into the theatre in the way promoted by the panic story (or 'myth', as Loiperdinger has it) of L'arrivée d'un train en gare de La Ciotat.

It is worth considering the difference between the cinematic capture of this train, and a hypothetical audio recording of it (a comparison with historical valence, given the key role of train recordings in Schaeffer's early work). At a very basic level, the two are similar: they are abstracted from reality via their respective mediatisation, and are in no way 'real' trains. However, audio recordings offer a sense of the real that visual recordings do not, beyond the phenomenological discussion of temporality addressed in the preceding section, and this is related to their method of playback. The cinema screen positions the abstracted reality of the train at a specific location, usually in front of the viewer at some distance. There is a distinction between the screen and the materials projected on it, and the space of the room, where a spectator sits remotely from the images. It is this remove that allows the spectator to easily distinguish between the action on the screen, and their present reality. However, audio playback blurs this distinction. It does this by utilising the architecture of the room of audition. Though the speaker(s) may also be placed in front of the spectator just as the screen is, the sound of the room becomes more pronounced the louder the amplification, emphasising the space of audition, thus wedding the sonic aspects of the past reality with the new reality of the theatre. ${ }^{66}$ As such, as the train approaches the camera, the room becomes sonically enlivened by it, at once announcing the presence of the room, and the sound of a train within it.

In contrast to this assertion, Michal Chion suggests that:

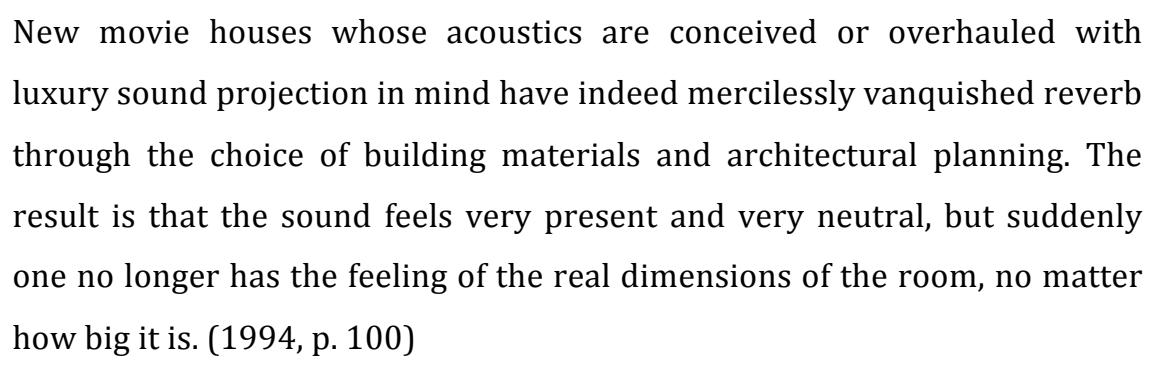

\footnotetext{
${ }^{66}$ Blesser and Salter (2007) address the role of architecture in aural experience. This notion sits within the basic premise of their thesis.
} 
Though Chion attests to the presence of sound in the modern movie theatre, he believes that the presence of the room has been muted. This may indeed be the aim of modern theatre design, but I argue that to say 'one no longer has the feeling of the real dimensions of the room' fails to account for the complexity of acoustic experience in such conditions. Chion's conclusion conceives of a room's acoustic profile as simply a matter of reverberation, and when such reverberation is removed, the theatre no longer "speaks". In fact, movie theatres have a very specific acoustic profile, a profile that is becoming increasingly common due to the adherence of many theatres to industry standards, ${ }^{67}$ and as such, not only do theatres "speak", but they speak with an increasingly standardised voice.

To further argue his point, Chion recounts the sound demonstrations typically found before the start of movies at industry-regulated movie theatres. Of the THX demonstration, Chion suggests:

\begin{abstract}
We may note two characteristics of this sound demo that typify current taste. First, the bass sound that the glissando ends on is clean of all distortion and secondary vibrations, even though very low sounds in the real world have the necessary consequence of causing small objects to vibrate - for example, a passing semi truck sets the furniture or the dishes to shaking. What the demo short is doing to stir the audience's admiration, far from any idea of fidelity, is showing off the technical capacity to isolate and purify the sound ingredients. Second, one finds no trace in the demo of the reverberation that normally accompanies and muddles loud sounds in an enclosed space. (1994, p. 100-101)
\end{abstract}

While this may be the case, I argue that these acoustic signatures, while lacking the overt expression of a reverberant concert hall, are nonetheless signatures that may be easily identified by listeners. As such, while the THX demonstration may attempt to focus our attention to the superior performance of the sound system by shortening reverberation times across the full spectrum of sound in the room, that is not to say the audience won't be aware of the space of audition. In fact,

\footnotetext{
${ }^{67}$ THX certification is one such standard common in theatres worldwide, requiring specific acoustical and visual performance from theatres and the technologies they use. See http://www.thx.com/professional/cinemacertification/thx-certified-cinemas/ (accessed 5 May 2014). Likewise, numerous theatres worldwide have adopted the theatre design specifications of Dolby Cinemas. Dolby Cinemas have announced (April 9, 2015) that AMC Prime Theatres will open more than 100 new theatres across North America with Dolby's new specifications. See http://investor.dolby.com/releasedetail.cfm?ReleaseID=905675 (accessed 9 June 2015).
} 
quite the opposite is true: that an audience does not hear rattling or muddled sounds reminds them that they are in the highly controlled acoustic space of a cinema. In other words, just because a cinema is not a reverberant space, does not mean an audience is less aware of it.

To determine this is indeed the case, imagine for a moment an audience sat in a movie theatre listening to the sound of train approaching without a visual referent. As the amplitude of the recording intensifies, the theatre responds in a manner directly related to its architectural profile. Though the cinema is a controlled space, it is nonetheless a specific and identifiable space to someone sat in it, not least of all because the acoustic profile is determined as much by the architecture of the room as it is by the number and composition of people within it. Simply put, a full theatre sounds different to an empty one. The most important thing to note is that to some extent, even a great extent, the acoustics of the cinema behave in much the same way as they would if a real train were approaching, depending, of course, on the quality of audio capture and playback. Increasingly, the quality of audio playback both at home and in cinemas is very high: often full range with dedicated subwoofers. Using Ingold's analogy as recounted in chapter four, as the train 'launches' into the medium of sound in situ, the loudspeakers of the cinema transfer this energy into the new space, and sense of 'the real' is achieved, with the new space behaving in ways appropriate to its acoustic profile, whatever that profile may be or the intentions of those who specified it.

This coupling of the medium of sound in situ with the medium of sound in the new environment, gives it a heightened sense of reality that eludes the visual coupling of light between the past event and the theatre. Though it might be argued that, in theory, cinema responds to changes in light from the screen in a similar manner to that of sound from speakers, in my view the results are quite different. When a train resounds throughout a cinema, the space is engaged. A listener becomes ensounded by the train in the theatre, giving the train a sense of presence. As such, the room simultaneously announces its own presence, while announcing the presence of a sound within it. Though it is beyond the scope of this thesis to thoroughly examine the differences between light waves and sound waves as 
Kock (1965) does, I will simply say here that moving images do not impart the same sense of presence and reality in the cinema that sound achieves as a matter of course. Though light may reflect, diffract and dissipate in a cinema space as sound does, the moving image is forever tied to the screen upon which it is projected. As Truax (2001) has it: 'the sound wave arriving at the ear is the analogue of the current state of the physical environment' (p. 17). At this basic level, light waves arriving at the eye from the screen do not provide us with the same level of environmental information, thus we are less aware of the cinema itself and the interplay of light within it, not least of all as our visual field is a front facing "fan" rather than the omnispherical field of sonic perception (Ihde 2007, p.75).

In summary, though the moving image and its sonic counterpart are of the same order in terms of their relationship to a past reality, sound asserts itself through a presence that is not found in 2D cinema. In this way audio recordings are perceived as both temporally and spatially present whereas visual recordings are perceived as spatially removed from the spectator (they occur 'over there, on the wall') with a level of temporal ambiguity; that is, without the convincing animation, linearisation and vectorisation of temporality as outlined by Chion.

\section{D film}

Though 3D films protrude into the theatre in a way that 2D films do not, the distinction between reality and fiction does not seem to have undergone any modification. Though the illusion of images invading the space between the spectator and the screen makes for a more thrilling experience, I believe the order of an audience's suspension of disbelief has not changed, nor does it approach the kind of experience afforded by sound. Unlike the audio recording, 3D film is still tied to the screen through the process of encoding and decoding, in which the resultant 3D image is in no way related to the 'current state' of the room or theatre in which the viewing occurs. As such, 3D film remains an optical illusion, while sound reverberating off walls presents a sonic reality, coupled with the playback of a past reality. Expanding on the ideas of the cinema's 'aural architecture' (Blesser and Salter, 2007) as discussed above, the surround sound 
format of many modern cinemas seeks to minimise the sound of the room just as the acoustic design of the room itself does, in pursuit of enhanced spatial 'realism'. However, no matter how complex THX or Dolby room specifications become, or any future format for that matter, listeners will always be able to hear reflections in the room and every nuance of the acoustic environment, such as the acoustic effects of seats, people, their popcorn boxes, the large screen in front and so on. As such, though the iconic thud of the tyrannosaurus approaching the car in Jurassic Park may envelope the audience through spatial distribution and loudness, the room still speaks, giving immediate presence to the thud. The surround format does not modify this sense of presence; rather it modifies the sense of space.

To conclude that 3D film alone does not blur the lines between past reality and present audition, we might look to the recent filmic realisation of J.R.R Tolkien's book The Hobbit. Imagining for a moment the film is silent, regardless of whether the fictitious dragon Smaug from director Peter Jackson's The Desolation of Smaug (2013) is viewed in 2D or 3D, it is likely considered no more or less real, just more or less thrilling. However, this is not to say that 3D films are unable to grant greater access to attributes of an unmediatised 'reality' as captured on film. Indeed director Werner Herzog, an outspoken critic of 3D films, shot his film Cave of Forgotten Dreams (2010), a documentary depicting the 32,000 year-old cave paintings on the walls of the Chauvet Cave in the south of France, in 3D:

I've never used the process in the 58 films I made before and I have no plans to do it ever again, but it was important to capture the intentions of the painters. Once you saw the crazy niches and bulges and rock pendants in the walls, it was obvious it had to be in 3D. (Herzog in Goldstein and Rainy, 2010)

For Herzog, 3D filming was essential for conveying a sense of spatial depth, but in general terms, he views 3D technology as largely distracting:

We shouldn't ever have a romantic comedy in 3D, because we, the audience, have an emotional approach to the storytelling which leaves open a lot of narrative possibilities...you wonder as you watch - will the young man and the woman find each other? Fall in love? We start to fantasize, which you 
could never do in 3D, where you would be in the handcuffs of the technological effects. With cinema, your fantasies should always be free. (Herzog in Goldstein and Rainy, 2010)

Wim Wenders, a director often associated with Herzog through their mutual association with the Neuer Deutscher Film movement, offers a different opinion of 3D film. In his film Every Thing Will Be Fine (2015), Wenders promotes the power of 3D to augment the film, helping to "bring out the emotions" of his characters:

I could see it created a whole different presence in close-up. It has a magnifying effect, it's like a magnifying glass, making everything stand out. (Wenders in Connolly, 2015)

Though Wenders' point of view may seem to refute Herzog's, from a poietic perspective Wenders' use of 3D in Every Thing Will Be Fine, is much like that of Herzog's in Cave of Forgotten Dreams, insofar as 3D is used to reveal the contours of the subject matter in order to draw closer attention to their respective properties. In Herzog's case, 3D is used to connect the audience to the motivations of the artist(s) by revealing the contours of the cave walls, while Wenders uses 3D to connect the audience to the motivations of his characters through magnification of their faces. It should also be noted that early reviews of Every Thing Will Be Fine suggest that the use of 3D may not have achieved the kind of magnification of character emotion that Wenders sought, with one reviewer referring to some of the performances, perhaps wryly, as 'shockingly flat'. ${ }^{68}$ Film critic Nicholas Barber notes that unlike his earlier film Pina (2011), in which 3D was used to enhance the spectacle of dance, Wenders 'does so little with the 3D that it only deepens the impression that Everything Will Be Fine is a missed opportunity. ${ }^{69}$ While the criticism of 3D in Every Thing Will Be Fine focuses around what it does not achieve, it is plausible that, as Herzog might have it, the use of 3D distracts audiences, perhaps unconsciously in this instance, from connecting with the characters in the way Wenders imagines.

\footnotetext{
${ }^{68}$ 'Every Thing Will Be Fine' is a Major Disappointment sourced at http://www.indiewire.com/article/berlinreview-wim-wenders-every-thing-will-be-fine-is-a-major-disappointment-20150210 accessed 8 April 2015 ${ }^{69}$ Barber, 2015: http://www.bbc.com/culture/story/20150211-is-james-francos-latest-a-bore
} 
In contrast to Wenders, Herzog wishes to contemplate a film at a remove, so that he may hypothesise about the past and future of the subject matter. He conceives of spectator 'freedom' as afforded by a kind of spectator dislocation, which, as Robert D. Romanyshyn might agree, is born of the notion of seeing that instates distance between the object and the self. ${ }^{70}$ Accordingly, the perception of distance between the self and the image, measured as the exact distance between the screen and the eyes, is the ingredient that allows a spectator to build narrative, reflect on the past and imagine the future. For Herzog, film - as an illusion - encourages interpretation, much as the novel does; it is a hermeneutic form. Herzog's criticism of 3D film suggests that by encroaching on the space between the self and the screen, 3D materials distract from this process; arresting us in cognitive 'handcuffs', which is to say that 3D movement commands attention, making a spectator aware of the present in a way that detracts from the quality of their noetic response. As such, we might conclude that there is indeed a parallel between experience of the phonograph and 3D silent film, in that they both command attention to the present. However, I believe, as Herzog suggests, that 3D film does so in a way that does not allow for contemplation beyond the present, whereas the phonograph retains this capability. Indeed, and as I will discuss in chapter six, this capability provides the phonograph with some of its most unique features.

\section{Movement, attentional focus, protensity and the photograph}

In light of the preceding discussion, I will assert here that 'the present' is perceived differently in aural and visual perception, as outlined previously by Ihde, and that our ability to shift temporal focus in aural perception is not an ability readily afforded visual perception. Returning to Ihde, we note that in order to be aware of temporality from a visual perspective, we must undertake a maneuver that amounts to a cognitive turn to a noetic phenomenon. In the case of his son's baseball moving towards him, the 'noematic appearance of successive time' results. As Ihde makes clear, it is movement that aids in our ability to engage with temporal flow from a visual perspective. 3D movement, that is the perception of movement between the screen and eyes must further

\footnotetext{
${ }^{70}$ See Romanyshyn, R. D. (1989).
} 
aid in this process, much like the movement of the baseball towards Ihde's face in his previously recounted example. However, though the audition of sound and the viewing of 3D moving images may produce temporal awareness, the order of temporal focus along the temporal span is quite different. As Ihde notes:

In visual perception, the shape of focal attention found its locus in a central core within the visual field...the gravitational shape of this visual focus is weighed in the center of this visual field as a phenomenological structure. (Ihde, 2007, p. 91)

Ihde's comment is underpinned by the idea that the attentional focus of visual perception involves a spatial center. Unlike aural attention, which involves a shifting temporal focus, visual attention involves a spatial focus, presumably also at the 'leading edge' of the temporal span (as his example of the baseball, above, suggested). In other words, though moving images may make us aware of the succession of time, we are not able to focus across the temporal span in the same way afforded in aural perception. Further to this, I maintain that with a relaxed or 'broad' temporal focus, aural perception may be allowed to reach back beyond the horizon of Husserl's retentions, into memories of past experiences. We might recall Voegelin's experience of generating 'morning-parkness' in 2008 at Waterlow Park in London. While listening broadly to the sounds of the park at dawn, she is able to reach back to her experiences of parks, which in turn shape her perception of it. ${ }^{71}$ In this process, Voegelin is aware of the current temporal span, and the sounds operating within it. Without paying particular attention to any one point along this span, she is able to turn her focus to past memories. In this way, Voegelin exhibits a dual focus: she is aware of the past in the present. This particular condition of listening provides the phonograph with a unique characteristic, allowing for a very particular kind of engagement, one in which a listener is able to attend to both the phonograph in audition and memories associated with it. In contrast, a duality of focus comes far less readily in visual perception.

\footnotetext{
${ }^{71}$ Augoyard and Torgue (2005) refer to the phenomenon of sounds evoking memories of the past as anamnesis (21). Additionally, they refer to a sound imagined, but not actually heard as phonomnesis (85). In the instance of Voegelin recalling her 'morning-park-ness' the sounds of Waterlow Park produce an anamnesis of her past experiences of parks.
} 
As I will recount in chapter six, Jacques Rancière (2013) discusses the 'brute presence' of the photographic referent, one in which our attentional focus is forever tied to that which is photographed. Herzog makes this same criticism of 3D film - we are 'handcuffed' to the visual effect - while Roland Barthes, as I will discuss shortly, believes this to be a condition of the moving image, regardless of the image's dimensional attributes. As these examples attest, to varying degrees visual perception is largely bound to the visual referent. The phonographic referent, however, is only one of many possible referents that a listener may engage with when listening to a recording. Though Barthes would argue the photograph too holds this ability, I believe that the phonograph compels a listener to engage with ideas, experiences and memories well beyond that which is directly presented in the phonograph. The photograph allows for such musing, though this engagement is forever tied to the referent, such is the power of its brute presence. In other words, while the photograph may produce an array of personal responses, such is Barthes' thesis, these responses are born of and wed to that which is photographed. The phonograph can evoke responses that, though born of the phonograph, are not necessarily wed to the referents contained therein. I will return to these points in chapter six.

With the preceding discussions in mind, we can consider the core reasons why film, whether silent, single shot, 2D, 3D or otherwise, is inapt for comparison with the phonograph. Though cinema and the phonograph both share a temporal dimension in that movement may be detected in both (not forgetting that sound is contingent on movement), the perception of moving images occurs at a fixed temporal and spatial location: in front of us, as Ihde has it, in the center of our visual field, and most importantly, at the edge of the protending future, without the ability to envelope us or easily marry itself to a new environment as sound does. Nor does it allow our attention to explore the temporal span of the present reality. Furthermore, visual perception arrests our attention in the present in a way that aural perception does not. Though Herzog ties this condition to 3D film, others, including myself, would argue that this is a condition of all film. Roland Barthes makes this contention in Camera Lucida (1981): 
Do I add to the images of movies? I don't think so; I don't have time: in front of the screen, I am not free to shut my eyes; otherwise, opening them again, I would not discover the same image; I am constrained to a continuous voracity; a host of other qualities, but not pensiveness; whence the interest, for me, of the photogram. (1981, p. 55)

Here Barthes levels the same criticism at 2D film as Herzog does at 3D film, though for Herzog his inability to adequately engage with the materials is born from a perceived invasion of space, whereas Barthes' experience is confounded by the fact that the images are moving, an invasion of his time. That the images are moving means, for Barthes, that real-time interpretation of film is not possible, as he simply does not 'have time'. As the above quote suggests, Barthes understands moving images to lack the ability to allow for a personal adventure to occur. As I will explore in chapter six, Barthes believes that the still image encourages a hermeneutic response, one in which a kind of personal adventure may take place.

Further to Barthes' assessment of the moving image as demanding 'continuous voracity,' he notes that film shares the same temporal progression as our standard perception of time:

Like the real world, the filmic world is sustained by the presumption that, as
Husserl says, "the experience will constantly continue to flow by in the same
constitutive style"; but the photograph breaks the "constitutive style" (this
is its astonishment); it is without future (this is its pathos, its melancholy); in
it, no protensity, whereas the cinema is protensive, hence in no way
melancholic (what is it, then? - It is, then, simply "normal", like life).
Motionless, the Photograph flows back from presentation to retention. (1981, p. 89-90)

Barthes' assessment suggests that the 'constitutive style' of film, that is, its likeness to real-time, is what makes it protensive. Such temporality impedes Barthes' interpretation, rendering film inferior to his engagement with the photograph, a medium in which he is able to detect pathos. It should be noted here that Barthes' film presumably involves the marriage of sound and vision, and in some respects his comparison of film and the photograph encounters the very stumbling block I have been at pains to avoid - the comparison of a mono- 
modal format with a multi-modal format. It is unclear as to how Barthes may have felt about silent film or indeed the phonograph, but we might be safe to assume that he would have detected the same 'constitutive style' in both formats, and on this basis, labeled them both protensive.

The word 'protensive', as employed by Barthes, is used to describe the reaching of the one point in time to another, in the case of film, from the past to the present. As mentioned, we might conclude that Barthes' may have considered the phonograph to convey the same protensity as the moving image, insofar as the constitutive style of a past reality is replayed in the present. Likewise Chion might argue that the protensity of the phonograph is even greater, given its power to define the present and the successive passing of time, the role it plays in the audiovisual contract. However, the protensity of the phonograph requires further analysis. To begin, Barthes detects melancholy in all photographs; that is to say, this melancholy is ontological, born of the medium itself. This can be detected in the viewer's movement between the present to the moment of capture. Barthes conceives of this movement as a spectator's attentional focus to 'the pose':

What founds the nature of Photography is the pose. The physical duration of this pose is of little consequence; even in the interval of a millionth of a second (Edgerton's drop of milk) there has still been a pose, for the pose is not, here, the attitude of the target, or even a technique of the Operator, but the term of an "intention" of reading: looking at a photograph, I inevitably include in my scrutiny the thought of that instant, however brief, in which a real thing happened to be motionless in front of the eye. I project the present photograph's immobility upon the past shot, and it is this arrest which constitutes the pose. (1981, p. 78)

Barthes continues, suggesting that it is 'the pose' that defines the photograph, separating it from the related practice of film:

This explains why the Photograph's noeme deteriorates when this photograph is animated and becomes cinema: in the Photograph, something has posed in front of the tiny hole and has remained there forever (that is my feeling); but in cinema, something has passed in front of this same tiny hole: the pose is swept away and denied by the continuous series of images: it is a different phenomenology, and therefore a different art which begins 
here, though derived from the first one. (1981, p. 78)

Accordingly, does the 'constitutive style' of the phonograph always result in the same impaired engagement Barthes ascribes to film? Does the phonograph attest that something has passed before the microphone, and is the phonograph, then, always protensive, reaching from the past to the present in the same manner as film, without a 'pose' to return to? Does the temporal flow of the phonograph demand the same 'continuous voracity'?

I argue that though audio recordings and films both direct an audience's attention to the present, they do so with different results. As Herzog suggests, 3D film attunes our attention to the present at the expense of our ability to ruminate; it is commanding to the point where there is no room for a spectator to 'fantasize' about the materials, which, for Herzog, is what gives film its value. Audio recordings, though commanding our attention toward the present as a key feature of auditory perception, do not encroach on our noetic ability in the same way. When listening attentively we are keenly aware of the present, yet we are also able to perceive of the past with ease, explore it, and bring it to bear on our noetic aural perception of the present. Our ability to do this while viewing a film is greatly dependent upon the kind of movement present on the screen. As Ihde suggests, movement in visual perception can result in a turn to the noetic phenomenon of passing time. When there is no movement on screen, we may be less aware of this 'constitutive style'. Imagine for a moment a static shot of New Zealand's southern alps (akin to a photograph). Such a shot may allow for a similar delving into past experiences, even a return to 'the pose', but this process is abruptly cut short as the camera begins to move, or if the objects in the frame become animated; we are now aware of time passing, and indeed our attention is now focused to not only the present, but to the protending future. This attentional command on a spectator's focus is even greater in films with strong narratives and those that are anthropocentric, as Barthes would have surely agreed. By comparison, that sound may be ever-changing, densely layered or fast moving is of limited consequence in our ability to perceive the past, as our ability to perceive sound and analyse it at speed, as suggested by Chion, is far greater than our ability to do so with moving images. James Joyce articulates this particular 
duality of auditory perception in Ulysses, while considering how a phonograph might act in a similar fashion to the photograph:

Besides, how could you remember everybody? Eyes, walk, voice, yes: Have a gramophone in every grave or kept in the house. After dinner on a Sunday. Put on poor great-grandfather. Kraahraark! Hellohellohello amawfullyglad kraark, awfullygladaseeagain hellohello amawf krpthsth. Remind you of the voice like the photograph reminds you of the face (Joyce in Toop, 2004, p. 107)

Though great-grandfather is clearly absent, his voice arrests the listener as if he were present, at the grave or the dinner table. Joyce suggests that while listening to his voice, he is also reminded of great-grandfather's eyes and walk. As such, Joyce describes an experience unique to the phonograph, which separates it from other experiences of mediatised reality: the materials encountered in the phonograph reach forward from the past, rendering the distance between the moment of capture and moment of audition almost imperceptible, insofar as the materials arrest our attention in present and engage with the auditory architecture of our environment. However, we are simultaneously able to 'fantasise' about the materials, which may include, at the most basic level, memories of the past as evoked by the materials in audition. An interesting relationship between the past and present is thus revealed. As noted by Barthes, where a photograph is concerned, the spectator is immediately drawn back to the moment of capture: the present reaches for the past as a photograph is encountered. Audio recordings present an opposing movement in that the materials immediately reach forward from the past to the present. However, having heard the materials in the present, a listener is able to reach back into the past.

Listeners are also afforded the mental space to 'fantasise' about the materials, in which they engage with the past reality of the materials while generating a vast array of unique responses. This is achieved through the broadening of focus along the temporal span, coupled with a broad focus on the materials in audition, which amounts to a collapsing of the distance between foreground and background materials. Therein lies the distinction between moving images and the 
phonograph: moving images are protensive, moving along in the 'constitutive style' of real-time, directing our attention without allowing much room for realtime contemplation, especially where the content is moving and changing rapidly. Phonographs, while protensive, also allow a listener to travel back to the moment of capture, a perceptual mobility which is inherent in listening. As I will argue in the following chapter, the phonograph also holds the unique ability to allow a listener to travel beyond the specific moment of the recorded event(s) to other corners of their memory. 


\section{6}

\section{Studium, Punctum, Anamnesis: toward an ontology of the phonograph}

Barthes' Camera Lucida (1981) provides a compelling account of the photograph's ontology from a deeply personal perspective. Given that my investigation into the phonograph's ontology places similar emphasis on the experience on the listener, his landmark work provides some interesting points of comparison. It is important to note that Barthes' analysis of photography is one of several interesting accounts, however, in my view, it is Camera Lucida that presents a broad and persuasive perspective on interpretation, such that its application can be extended well beyond the terms within which it was conceived. Indeed Douglas R. Nickel, while noting Camera Lucida's 'stature as the most sustained and thoughtful contemplation of a vernacular photograph extant in the literature', suggests that 'the author elaborated a hermeneutic model that opens up possibilities for analysis of a much greater range of non-art objects and practices that has heretofore been the province of traditional photographic history' (2000, p. 232). I share Nickel's perspective, and it is on this basis that I extend Barthes' perspective on the photograph to that of the phonograph. It should be noted here that Barthes' work is fundamentally a hermeneutic analysis of the photograph, as Nickel suggests. However, there are also aspects of phenomenology that can be seen to appear in his work, and while I will be focusing primarily on Barthes' analysis of interpretation, I will also look at how experience influences his writing. As I will argue, there are some features of Barthes research that are easily applicable to certain phonograph types, and here 
his ideas require very little modification. However, my deeper purpose is to explore how Barthes' analysis of the photograph might inform and extend what is known about the phonograph, in order to help build a more complete understanding of the nature of listener engagement.

Camera Lucida, is divided into two parts. Part One constitutes Barthes appraisal of his own engagement with the photograph, exploring its affective qualities. In Part Two, Barthes finds a picture of his mother, who had recently passed away, and declares it the photograph that conveys her air where all other photographs fail. This photograph becomes the central focus of this second section of the book, which constitutes a pursuit of the photograph's universal (ontological) qualities. Both sections provide excellent points of comparison with the phonograph, and it is to Part One that I will first turn.

Of Part One, Jacques Rancière notes:

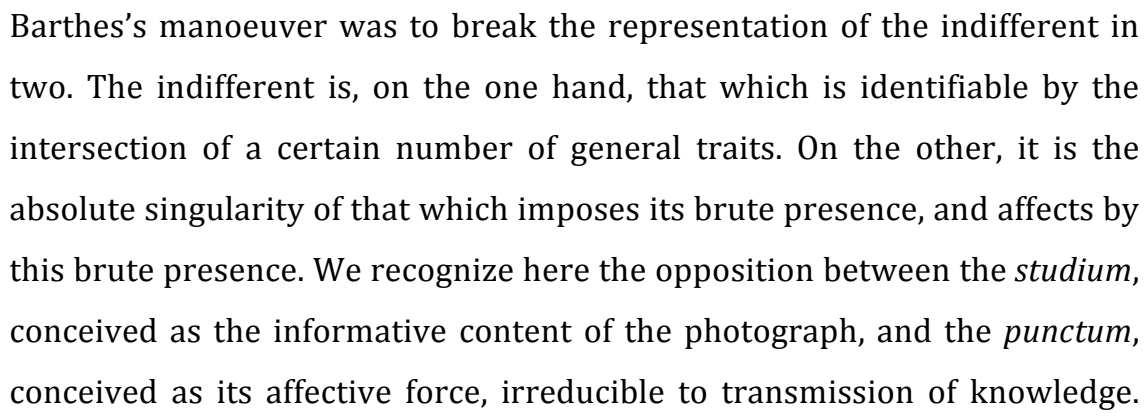
(2013, p. 87)

Barthes investigates the studium and punctum in some detail. He suggests that the term studium 'doesn't mean, at least not immediately, "study", but application to a thing, taste for someone, a kind of general, enthusiastic commitment, of course but without special acuity' (1981, p. 26). Of his experience of the punctum, Barthes suggests that it 'will break (or punctuate) the studium. This time it is not I who seek it out (as I invest the field of the studium with my sovereign consciousness), it is this element which rises from the scene, shoots out of it like an arrow, and pierces me' (p. 26). Such an appraisal may at first appear rather simplistic; Barthes' distinction seems to 
make an argument for a differentiation between the 'general traits' of a photograph and its affective properties, which it does to a certain extent. However, the implications of such a manoeuver reach much further than a simple intellectual/emotional, head/heart description of engagement. Rancière again:

In a certain manner, Barthes contorts the formalist modernist, who opposed the form (artistic/pictorial) to the anecdote (empiricist/photographic). Barthes diverts the opposition by transferring the anecdote to the studium, in order to pit it against not the artistic form, but an experience of the unique that refutes the pretension to art as well as the platitude of information. (2013, p. 87)

This reassessment of the 'formalist modernist' approach, in which the form and anecdote are grouped and juxtaposed with the unique experience of the individual viewing the photograph is of considerable significance when evaluating the phonograph, especially where environmental sound composition is concerned, insofar as the tension between form and anecdote is, as I have argued, a significant point of contention between environmental works and the broader genre of acousmatic music. As I will explore, if we are to uphold the notion that the form and anecdote of the phonograph belong to the same order (the studium), the tension surrounding the use of mimetic materials in electroacoustic composition is subverted. The focus moves from the materiality of the phonograph and the poietic process of the composer to the affective qualities of the work: qualities that are solely governed by the esthetic process of the listener. It is my strong belief that there is much more to be learned about the phonograph's ontology from a hermeneutic perspective when composerdriven poiesis is removed from consideration. This opinion finds support in Barthes' early assessment of engagement with the photograph in Camera Lucida.

There are two important observations made by Barthes in the early stages of his discussion, before his description of the studium and punctum begins. The first observation is the notion of adventure whereby Barthes' engagement with a 
photograph is contingent upon being animated by it (1981, p.19). ${ }^{72}$ The second is the notion of the Operator (the photographer) and the Spectator (the person attending to the resultant photograph) and how their perspectives differ. These two observations help to understand the terms within which Barthes' assessment operates. It also allows us to consider how his appraisal of the photograph might be further expanded.

Barthes notes that the world is flooded with photographs, and that he simply does not attend to all of them. We might say that world is also flooded with phonographs, as I'm sure those who promote Schafer's 'schizophonia' would agree, and that we too, in our selective listening practices, cannot attend to every recording we encounter. Barthes relegates the majority of photographs to that of objects without affect. For Barthes, these photographs do not exist, for they do not produce an internal advenience (adventure, animation), understood as the primary condition by which any kind of engagement with the photograph can commence:

The principle of adventure allows me to make Photography exist. Conversely, without adventure, no photograph. (1981, p. 19)

Barthes here exhibits what I take to be a phenomenological perspective, and it is in the notion of the picture that advenes that we discover the importance of Barthes' intentionality in the assessment of the phonograph's ontology. Barthes' 'adventure' is not chiefly a result of his cognitive interpretation of a still image, rather it is a product of his primary engagement with a photograph, one that catches him unaware:

In this glum desert, suddenly a specific photograph reaches me; it animates me and I animate it. So this is how I must name the attraction which makes it exist: an animation. The photograph itself is in no way animated (I do not believe in "lifelike" photographs), but it animates me: this is what creates every adventure. (1981, p. 20)

72 Barthes uses the terms animation, adventure and advenience interchangeably throughout his discussion. 
In phenomenological terms, Barthes insistence that a photograph only exists if it advenes, echoes Husserl's phenomenological concept of the intentional object (noema) as a product of noesis (intentionality). It also resounds within the context of reader-response theory, which Barthes contributed significantly to, a theory in which the audience or reader constructs meaning from the work, not the writer (Barthes, 1967). We might also recall Nattiez' semiology, particularly regarding Pirandello's observation that the reader "fills up" words with the meaning they have for them, as previously quoted in chapter four. As such, Barthes' insistence that a photograph's existence is contingent upon his intentionality finds support in a number of different theoretical fields.

It is from this position, one in which adventure is aroused, that we may begin to detect the affective features of the phonograph, including the studium and punctum. Barthes' initial observation is used to define the three kinds of photographs we encounter: those without adventure, those with adventure and, necessarily, a discernable studium, and finally those with adventure, a studium, and a punctum. The most important revelation of Barthes' advening picture is this: access to the ontological features of the photograph is only granted through the intentionality of the one who attends to it; that is, the photographer does not hold the key to the photograph's ontology, the intentionality of the Spectator does.

In an earlier essay entitled The Death of the Author (1967), Barthes promotes a reader-response theory that sets the foundation for this argument. Of traditional models of interpretation, he notes:

The explanation of the work is always sought in the man who has produced it, as if, through the more or less transparent allegory of fiction, it was always finally the voice of one and the same person, the author, which delivered his “confidence." (1967, p. 2)

Barthes dismisses this appraisal of the work and the presence of the author within it, stating that 'language knows a subject, not a person' (p. 3), and that the notion of interpreting the author's intentions in text is redundant: 
Once the Author is gone, the claim to "decipher" a text becomes quite useless. To give an Author to a text is to impose upon that text a stop clause, to furnish it with a final signification, to close the writing. This conception perfectly suits criticism, which can then take as its major task the discovery of the Author (or his hypostases: society, history, the psyche, freedom) beneath the work: once the Author is discovered, the text is "explained". (p. 5)

Barthes' appraisal of the author promotes a semiology of written language in which the author's poietic processes can be largely disregarded: for Barthes, the author is 'dead'. I will discuss the role of semiology in Barthes perspective in greater detail shortly with regard to the role of the operator and spectator, but for now I will note that in Camera Lucida, Barthes' analysis of the photograph also mutes the poietic processes of the photographer, proclaiming instead that the esthetic processes of the spectator are the most relevant in the activity of interpretation. He states: "certain details may "prick" me. If they do not, it is doubtless because the photographer has put them there intentionally" (1981, p. 47). Though Barthes' comment comes across as a thinly veiled ridicule of operator intentionality, he does not go as far as to say he can detect what such intentionality might be; to do so would be to hold that poietic processes do in fact feature in esthetic interpretation. What is revealed here is that Barthes believes a photographer's intentionality does not hold the power to inflict a punctum. Such a power is born solely of a spectator's personal response.

Regarding Barthes' second observation, in the opening pages of Camera Lucida, he notes 'that the photograph can be the object of three practices (or of three emotions, or of three intentions): to do, to undergo, to look' (1981, p.9). Of these three practices he describes the participants as the Operator (the photographer) the Spectrum (that which is photographed) and the Spectator (the viewer of the rendered photograph). Barthes notes that the operator's photograph is fundamentally different to that of the spectator, as the operator is concerned with the 'formation of the image through an optical device', while the spectator's experience is 'descended essentially, so to speak, from the chemical revelation of 
the object' (p. 10). Barthes' distinction recalls Molino's semiological tripartition of the poietic, neutral and esthetic levels (discussed in chapter four). It is important to note that Barthes concerns himself chiefly with human subjects in his analysis. Indeed of the 24 photographs reprinted in Camera Lucida, only one photograph lacks a human in the frame. His fixation with human subjects in his discussion is tied to a conception of the middle state, ('to undergo') as one concerned with mortality. Of having his own picture taken, Barthes notes:

I am neither subject nor object but a subject who feels he is becoming an object: I then experience a micro-version of death (of parenthesis): I am truly becoming a specter. (1981, p. 14)

The spectrum as spectre recalls a term employed by Nattiez to describe the neutral level of Molino's semiological tripartition, the trace:

We employ the word trace because the poietic process cannot immediately be read within its lineaments, since the esthetic process (if it is in part determined by the trace) is heavily dependent upon the lived experience of the "receiver." (1990, p. 11)

The notion of the photograph's trace as an emanation of a past reality, one in which the certainty of the moment passed is observed in a frozen and disembodied image, is a notion picked up by Dugal McKinnon, who insists that the phonograph too holds this ability:

Likewise, in the recording, sound spectra are preserved so as to allow departed but audible presences to emerge from black boxes: spectres from spectra. (2006)

It should be noted that, in case of Barthes 'becoming a specter', his transformation is in fact a poietic process; a kind of negotiation or performance between himself and the operator as the finger moves to the button that governs the operation of the shutter. The same might be said of audio recordings involving human beings, particularly musicians who strive to produce their best performance for the microphone as a matter of preservation; the phonograph 
"lives on" once the performer has passed away. Barthes' account of death in photography is complex and pervasive throughout Camera Lucida, and I will return to this topic in greater detail later in this chapter as it pertains to the phonograph. For the moment, I will note that Barthes' neutral level is largely tied to notions of death: as the shutter clicks, the spectrum is produced, and that which 'has been' is preserved. For the operator, access to this death is through the 'optical device' with a level of culpability, while the spectator's access to this death is one of chemical (or digital) revelation, a past reality now somehow present. I follow McKinnon in suggesting that phonography too presents this trace (indeed, I have identified it as an essential feature), though as I will discuss later in this chapter in relation to listener engagement, and alluded to in chapter four, it is not the only trace found in audio recordings. There is also a technological evaluation of the neutral level that is worth some consideration. On this basis we can see a further commonality between the photograph and the phonograph insofar as the neutral level ('to undergo') is a transitive and technologically driven state. Just as the sonic signatures of audio technology imbed themselves in a work at both ends of the transduction process (and indeed in the medium), so too do the visual signatures of the camera and the viewing medium, be it paper or pixels.

Making a distinction between the operator's photograph and the spectator's photograph is important. For a photographer, their choice of camera and lens, along with their knowledge of their camera's technical operation and performance is unique to their experience. As quoted above, they are still primarily concerned with the 'formation of the image through an optical device'. However, a spectator familiar with photography may exhibit an awareness of such technology, and though they will never share the same experience of the technology as that of the operator, engagement with their technological practices; that is, an intentionality that seeks out the technical production features of a work, may be a primary feature of their engagement. In other words, a spectator may too be interested, albeit at a remove and with a high level of subjectivity, with the same 'formation' of the photograph at a technical level. Such an esthetic response may even match poietic intent, though as Nattiez 
would agree, such a transaction does not constitute a transmission of intention, rather, a chance alignment of intentionality at the neutral level. Where Barthes is concerned, no such intentionality is expressed. Barthes claims that his assessment of the photograph excludes any recourse to that of the perspective of the operator. However, had Barthes acknowledged the presence of the operator, even as one who necessarily exists in order to facilitate a spectators' experience by operating the shutter, his assessment of the studium and punctum may have taken on some additional dimensions. It is my view that these technological dimensions are important to explore, not least of all given the proliferation of photographic, and phonographic, technology since the 1980s. While we might muse that Barthes himself would have considered engagement with a photograph's technological realisation on any level a feature of the studium, that is not to say that others may not be 'pierced' or 'wounded' by such an engagement. As I will later explore, a spectator's imaginative engagement with the operator's photograph is also exhibited in phonography, providing a rich field of engagement that is not explored in Camera Lucida.

Moving now to Barthes' studium and punctum, it is useful to detail his specific examples in order to reveal how they function. Let us consider for a moment, as Rancière does, Barthes' reaction to Lewis C. Hine's photograph Idiot Children in an Institution (1924). The photograph, showing 'two retarded children [with] monstrous heads and pathetic profiles' reveals the punctum, for Barthes, not in their unusual physiques '(which belong to the studium)', but in their attire: 'the little boys huge Danton collar, the girl's finger bandage' (Barthes, 1981, p. 51). For Barthes, it is this type of obscure detail that constitutes the punctum, which he describes as 'that accident that pricks me (but also bruises me, is poignant to me)' (p. 27). Of this designation, Rancière notes that 'the punctum, thus marked, in fact follows the same formal logic as the repudiated studium. It concerns, in both cases, features of disproportion' (Rancière, 2013, p. 87). Rancière makes a valid point, highlighting the lack of formal distinction between the two concepts in Barthes' example: as such, we must understand the designation of studium and punctum as entirely dependent on the interpretive process of the spectator. Barthes does not refute this, stating 'it is not possible to posit a rule of 
connection between the studium and the punctum (when it happens to be there). It is a matter of a co-presence, that is all one can say' (1981, p. 42).

Turning now to the phonograph, a fundamental question must be asked: do we detect a duality between the affective and the formal/anecdotal when attending to audio recordings? In other words, does my engagement with the phonograph produce a studium and punctum as the photograph does? In my view, such a duality does indeed exist. It is important to reiterate that I find the notion of the studium and punctum compelling, and like Nickel, see this as a readily translatable hermeneutic model. I also believe that the studium and punctum can and should be greatly expanded within the territory of phonography. Before exploring the diversity of the punctum, I will first consider a simple example, in which, like the Danton collar of Hine's Idiot Children in an Institution (1924), the punctum is understood as a detail, perhaps even an unintentional one.

\section{Punctum as detail}

David Toop's album Black Chamber (2003) contains three environmental sound compositions of a kind often associated with phonography (considered as a genre); single-take recordings with limited or no editing. Silver Birds is one of these tracks. For the 2'24" duration, Toop quietly moves through a night market in Chiang Mai with his recorder. The piece begins abruptly with a version of Felice and Boudleaux Bryant's “All I Have to Do is Dream" (1958) playing on a radio, which quickly fades as the incessant sound of electronic trinkets take over, including the crowing of electronic roosters and a device with a repetitive, modulating high frequency oscillator. All the while, small engine motorcycles and scooters strain past and barely audible excited conversations can be heard. The audio quickly fades out at the end. It is a hectic piece, and strangely familiar.

First and foremost, for me, this phonograph advenes. Perhaps this is a result of the level of activity in the materials, or perhaps it is because I have also experienced night markets in Thailand, but for whatever reason, I am animated by this work. The studium for me resides with the diversity of the human 
activity, the interesting sounds of the market gadgets, the traffic, conversations, and perhaps Toop's intentionality; though I can never know for sure, I might assume that, at the very least he found the environment sonically stimulating, which fueled his desire to record and present it. If we are to focus on Toop the composer we might recall Smalley's notion of transcontexts, in which the composer intends that we are aware of the environmental context and a new compositional context created from it, though in this instance, such an evaluation seems of little value. The composer, in this work, does not feature strongly and any consideration of his intention for me, rests with the lesser affecting force of the studium; it is one of several 'general traits' of the piece. The punctum of this work arrives at $0^{\prime 2} 25^{\prime \prime}$. A male voice calls to his companion in Japanese, and is then immediately gone, disappearing from the scene. From this moment the phonograph takes on a different hue. The fleeting sound of a Japanese tourist in a night market may not produce a punctum for many, but it does for me.

I, like many young foreigners who have lived in Japan, developed a deep fascination with Japanese culture (I worked there for two years in my early twenties). In my mid-twenties, I was involved with the Peace Boat, a Japaneserun NGO that sails approximately 1,500 Japanese people west around the world beginning and ending in Kobe, stopping in various ports, assisting with community activities, and engaging in cultural exchange. I was a guest speaker and musical director on this boat between Jamaica and Hawaii for five weeks in 2006. When I hear the Japanese man call to his companion in Toop's piece, I do not simply identify a Japanese tourist, and relegate that presence to the studium, rather, I recall the kind of people I travelled with, often very eager and excited young Japanese people just out of high school, undertaking an unusual voyage outside the boarders of their country. I recall a pronounced negative stigma attached to the Peace Boat from the Japanese public, and indeed a negative stigma towards anyone taking extended absences from Japan and working life more generally. Many of the people I met on board the Peace Boat were rebellious in this regard, and many more simply did not fit in to Japanese life at home. The Peace Boat represented a kind of cultural freedom for so many 
people, and it is this freedom that I detect in Silver Birds, from a small and undoubtedly unintended moment. This phenomenon is what Augoyard and Torgue would describe as anamnesis (2006, p. 21), a phenomenon I will return to with greater analysis later in this chapter. However, this is not simply a matter of reflection, though reflection is often a feature of phonographic listening. In the true spirit of Barthes' punctum, I am bruised by the voice. It is not a feature I seek out, but rather one that pricks me.

In many respects, the punctum is the most easily translatable feature of Barthes' hermeneutic model, and particularly so in this instance, as the moment at 0'25" is quite definitely a detail, fleeting and inconspicuous against the louder, more dominant material. For me, my fascination with the spectromorphological properties of the materials, my appreciation of the composer and his choices, his use of technology and the sonic signatures they impart, and also my recollections of similar Thai markets (I experienced a number of them in 2002), all belong to the studium of Silver Birds. Of the temporal distinction between photography and phonography, I will simply note that, in my view, the fact that the photograph presents the studium and punctum together in an instant does not mean that simultaneity is required for their respective detection. They are not limited to the photographic medium. Temporality does, however, play a part in the ability for a phonograph to advene.

\section{Advenience in the phonograph}

As noted previously in this chapter, Barthes believed that his engagement with the studium and possibly punctum of a photograph is contingent upon being animated by it. I believe this to be true of the phonograph also: for the most part, recordings pass us by in everyday life without producing any kind of engagement or adventure. However, I believe that the phonograph holds a greater ability to animate than the photograph, when they are being attended to. As I flick through the images reprinted in Camera Lucida, I might view 24 images in as many seconds, quickly deciding that all 24 images are without advenience. Though my engagement with each image is fleeting, I am 
nonetheless able to engage fully, as the content is all there, already there and always there. Though it might be argued that a photograph requires more than one second to be fully attended to, the amount of time required to observe the photograph is not dictated by the photograph itself; this is the prerogative of the spectator. 24 phonographs, however, require a much greater investment of my time: I cannot claim to have heard a 3-minute phonograph unless I have listened to all of it. Unlike the photograph, attentive engagement with the phonograph requires a fixed span of time. The vast majority of phonographs that do not advene in daily life are those that are fleeting and heard in part; a passing car with the radio turned up, or a largely inaudible stereo in a busy café, for example. However, if one attends to a phonograph in its entirety, it will more often than not produce advenience, even if it is of the most mundane variety. This is due to the demands on our attention over time. Not only does the phonograph have a greater opportunity to advene given the greater amount of time dedicated to it, but the 'constitutive style' of time passing observed in the phonograph may also correlate to the constitutive style of advenience: as the recording moves from one moment to the next, so too does the internal adventure of our response. In other words, the phonograph has the ability to hold our hand, so to speak, on an adventure that the photograph does not, despite having no control or understanding of what our adventure may be. This leads me to my next point.

The presence of the phonograph, that is, the ability for the past reality of a recording to engage in the present, both spatially and temporally, also promotes an adventure that the photograph does not. This is in part related to the 'constitutive style' as mentioned above, but also as a result of the phonograph's ability to physically alter the state of the environment's air pressure and velocity, and the listener's intermingling with it as per the discussion of Ingold's ensounding in chapter five. With these aspects of the phonograph in mind, understood in general terms as its spatio-temporal demands on our attention, an internal animation can be observed that is not apparent in photography. For this reason, I believe that an engagement with the studium and possible punctum of a phonograph comes more readily than that of the photograph. 
That is not to say that all phonographs, listened to in their entirety with attentional focus, produce a high level of adventure, a deep engagement or an enlivened internal animation. I suspect that the opposite may be true; that the phonograph so often results in adventure may result in a kind of experiential numbing for some, in which more often than not, listeners are only concerned with the aspects of a phonograph found in the studium. In many cases, experiential numbing may come as a result of a dominating human voice in audio recordings focusing our attention to matters of language, an idea I will further pursue shortly. For now I will state that though advenience comes with some ease to the phonograph, the animation may be of a superficial variety, and that the ability to produce a punctum is limited as a result. We might consider the genre of muzak as being a musical genre that exploits this quality: the listener animation in response to muzak is hoped to be limited, the music designed to impart a sense of mood and pace (light and slow), with only the slightest engagement with the studium (a familiar tune, a soaring string section) with no desire to inflict a punctum: the true aim of muzak is one of commercial coercion, not of penetrating artistic or affective engagement. Barthes' refers to photographs without a punctum as 'unary', and muzak is a prime example of the unary phonograph, if they are heard at all. For many, muzak does not advene, indeed Barthes might have said it does not exist.

\section{The unary phonograph}

I would like to provide an example of the unary phonograph where environmental sound composition is concerned, as it helps to illustrate not only the phonograph with studium but without punctum, but it also illustrates how a composer's intentionality can fail to deliver the desired result to a listener on a hermeneutic level. I take a work I have referred to previously in this thesis: Hildegard Westerkamp's Kits Beach Soundwalk (1989). As recounted earlier, Kits Beach Soundwalk is an exemplary work of soundscape composition, with Westerkamp admonishing the sounds of the city, while promoting the sounds of nature and her 'inner world of listening' (Westerkamp in LaBelle, 2006, p. 209). 
For me, Kits Beach Soundwalk advenes. My animation is chiefly a result of my attentional focus, and as someone who regularly listens to a variety of recordings with a critical ear, this is not unusual: for me, most phonographs advene. The recording interests me beyond this though, largely from a technological perspective; which microphones were employed, which stereo configuration, which recorder, how close to the source, which filters, and so on. Westerkamp exhibits in this work and many others, a deft touch with field recording and studio production, and it is this aspect of the work that engages me the most. In many circumstances a technological engagement with a work may produce for me a punctum, but not in this case. The opportunity is removed with the arrival of Westerkamp's voice, which embarks on a running commentary, describing what is heard, and later, the very studio technology and techniques my ear strains to hear. Westerkamp's commentary interrupts my ability to traverse the temporal span of the phonograph. As is no doubt Westerkamp's intention, my ear is drawn to her voice, and as a result, to the futural edge of the work, scanning the words as they arrive so that I may hear and anticipate what she has to say. The other qualities of the sound environment become the background of my sharp attentional focus, and had her voice not been present, I may have been able to explore the sounds of the barnacles and the high frequency content that make up the majority of the piece; an exploration that Westerkamp, ironically, is at pains to promote.

Westerkamp's voice confounds my experience on another level. The content of the words, as described, serve to promote her poietic intentions, in an attempt to make clear what her work is about, and possibly to assist, as Truax would have it, with the 'reintegration of the listener with the environment in a balanced and ecological relationship' (2001, p. 240-241). Though it is the presence of her voice, not the content of her words, that is the inhibiting factor in my experience of her piece, it is nevertheless important to acknowledge the role of her voice as a dramaturgical device within the piece. As I have argued in chapter four, my contention is that soundscape composers fail to meet their own criteria for successful works on the basis that their hopes for listener 'reintegration' rest on a poorly conceived semiological and educational model. 
For me, Westerkamp's words achieve the opposite of their intended function. They do not 'reintegrate' me with the environment; rather, they alienate me from it. We might recall again Barthes' comment that if details fail to 'prick' him, 'it is doubtless because the photographer has put them there intentionally' (1981, p. 47). Likewise, in this instance, I remain unmoved by Kits Beach Soundwalk due, in part, to Westerkamp's desire to move me; this phonograph is, for me, unary: a work without punctum. As such, there is no formal distinction between my interpretation of this work, and the majority of muzak. While I'm sure any composer would be outraged to have their work compared with something as insidious as muzak (soundscape composers particularly, and Westerkamp especially so, given her own research into the genre), I do not intend to offend, rather to bluntly illustrate a point: the composers of muzak operate with didactic intent; they intend that I should be affected in a certain way, though I remain largely unaffected. In Kits Beach Soundwalk, Westerkamp, to once more quote Voegelin, exhibits a more 'didactic composing at the listener' (2010, p. 32), and I also remain largely unaffected. In other words, it is Westerkamp's demands on my attentional focus and intentional response that stop me from exploring the work in the way she prescribes, and as a result, there is nothing about this work that deeply affects me. In this way, I too exhibit a bias found in Barthes. It is the explicit intentionality of the artist, the aspects they invest with the greatest meaning that move me the least. Not all soundscape compositions produce this effect for me (as I will later articulate), but what I am trying to make clear here is that for me, heavy-handed poietic contextualisation can have the effect of hindering the advience, studium and punctum of engagement with the phonograph in general terms.

It is important to note that my engagement with Kits Beach Soundwalk does not constitute a re-division of the anecdotal and the formal in my appreciation of the phonograph. Though Westerkamp's voice is clearly 'anecdotal', it is primarily the presence of her voice that renders the piece unary, not the words she recites. As such, I find most, if not all pieces with narration to be unary: audiobooks and radiophonic works (with narration) do not engage me (produce adventure, reveal a punctum), on the same level as phonographs without 
narration. As such, had Westerkamp presented her piece without narration, I would have been able to (potentially) experience a studium and punctum, though the anecdotal, environmental sounds of Kits Beach remain.

\section{Punctum as death}

Returning now to the varieties of punctum described by Barthes, I would like to address the notion of death in the phonograph as alluded to previously in this chapter. Recalling Barthes' analysis of protensity, he holds that photography presents a melancholy that is not observed in other forms of mediatised reality. In his view, the photograph is not protensive, in that the moment of capture does reach forward to the spectator as cinema does. Rather, the spectator is drawn to the past, to 'the pose'. Here Barthes articulates his conception of the photograph's essence (its noeme) with clarity, distinguishing it from film, and indeed phonography, as a matter of temporal incongruence. Barthes' promotion of the pose in the Photograph leads to the detection of death:

\footnotetext{
By giving me the absolute past of the pose (aorist), the photograph tells me death in the future. What pricks me is the discovery of this equivalence. In front of the photograph of my mother as a child, I tell myself: she is going to die: I shudder, like Winnicott's psychotic patient, over a catastrophe which has already occurred. Whether or not the subject is already dead, every photograph is this catastrophe. (1981, p. 96)
}

Can the same be said of the phonograph? I believe Barthes would suggest not, due to the nature of its temporal flow. However, he does concede that he 'can never see or see again in a film certain actors whom I know to be dead without a kind of melancholy: the melancholy of Photography itself (I experience this same emotion listening to the recorded voices of dead singers)' (79). This raises an interesting question. Barthes experiences a kind of photographic melancholy when hearing dead singers, therefore can we conclude that melancholy is detected in every phonograph in which the people recorded are know to have passed from existence? Barthes' observation is presumably based on the notion that in this instance, the phonograph brings the dead to life, and it is this 
confrontation between the deceased (in a 'life-like' state) and the listener in the present that promotes his melancholy. Unlike the photograph, in which melancholy is tied to its noeme, I do not believe we can make the same argument for the phonograph on the basis that the deceased no longer exist, or that they are destined for extinction. However, though death is not an ontological feature of the phonograph this is not to say that we cannot experience the kind of melancholy born of the knowledge that someone has passed on. Unlike the photograph however, this melancholy is a matter of degree, and I contend, in the most severe instances, far more potent than the melancholy of the photograph. This potency derives from a coupling of the past and present through the medium of sound, a coupling that does not occur in the photograph and its viewing.

Bing Crosby died in 1977, two years before I was born. When I hear his version of "I've Got the Girl!" recorded in 1926 with Don Clark and His Los Angeles Biltmore Orchestra, I do not experience any melancholy whatsoever, though I know him to be dead. For me, Crosby's voice enlivens the room in which I sit, though his voice emanates from the past, and though the recording must surely hold spectre-like qualities, his death is not embodied in this recording, nor the deaths of the Los Angeles Biltmore Orchestra. Even singers I have known to be alive in my lifetime who have since passed away do not necessarily produce this affect. However, in cases where I was once invested in a singer's (or musician's) work while they were alive, who have since passed away, I experience a melancholy, with the melancholy becoming more pronounced the more invested I am. For example, upon hearing recordings of artists such as Kurt Cobain (d. 1994), or rapper Christopher Wallace (aka Biggie Smalls, d. 1997), tracks produced by James Yancey (aka J Dilla, d. 2006), or Michael Jackson (d. 2009), I experience the kind of melancholy Barthes attributes to the photographic image. For me, listening to the recorded voices of those you knew in person, that is some one you knew in a personal context, and shared unmediated sonic space with, produces an entirely different affect. 
In 2001, I was the bass player in a rock group that performed around

Wellington. The project was brief; we only played together a few times before we disbanded. We produced one recording, a live concert of five tracks performed in early 2001 at a local venue. A few months after this recording was made, our vocalist went missing, and was eventually found dead, her life taken by an abusive partner. Needless to say, the recording we made is difficult for me to listen to. Unlike recordings of dead people I have never met, this recording is deeply unsettling. When I hear this recording, her voice, moving air particles in the room as they once did in person, is spectre-like in a way the eludes the photograph. I recently viewed a photograph of her, one that was displayed at her funeral, and though I experienced melancholy, a bruising punctum as Barthes might have it, it is nothing like the bruise inflicted by the sound of her voice in the phonograph. Where my mind returns to the pose of her photograph, her voice reaches forward in the phonograph, at once collapsing the distance between the past and present, yet simultaneously instating a clear division between her audible presence and her departure some 14 years ago. In this instance, the punctum as death is acute, far more so than anything I have experienced in photography. ${ }^{73}$

\section{Punctum as technology}

I have mentioned the role of audio technology in the reception of the phonograph in numerous contexts throughout this thesis, specifically with regard to the phonograph's sonic signatures and the role technology plays in the production of various phonograph types. In this context, I would like to consider technology as an element of the phonograph's trace, and as a specific type of punctum. As a recording engineer, the technological realisation of a phonograph, through all levels of transduction, is a primary concern for me. I am interested in both the technology of the sounds captured in a recording, including instrument

\footnotetext{
${ }^{73}$ It is important to note that this is not an isolated punctum for me. I have recently encountered a similar experience upon calling the household of a man who had recently passed away, his voice still emanating from the answering machine down the telephone line. I experience this punctum too when listening to New Zealand composer Jack Body's piece The Street Where I Live (2007). Having passed away earlier this year, his voice produces a melancholy that I am sure is experienced by many of his friends, colleagues and students.
} 
design, acoustic space and environmental concerns, and also recording technology itself; including microphone types, preamplifier specifications, storage mediums and playback systems. I am also interested in the acoustic profile of the playback environment. In other words, I am most often fixated on the signal path of a phonograph, from the first oscillation of a moving body in situ to the last oscillation detected by the eardrum of the remote listener. It is with this perspective that I approach the vast majority of phonographs. My perspective is perhaps one shared by many audio engineers, though the specificity of my engagement is entirely unique, as are theirs. Some would argue that my engagement is of a specialist variety, in which I have access to aspects of the phonograph that others may not, but this is untrue. I engage with features of the phonograph that are accessible to all, but given my background, I am inclined to hear that which others may relegate to secondary or tertiary features of the recording. As such, a specialist ear is not one of privileged audition (one hears something that another cannot), but rather a matter of attentional focus, born of a certain set of intentional concerns. In many respects, such intentional focus bears similarity to Schaefer's reduced listening and the environmental listening practices of soundscape composers. However, while these listening practices promote the intrinsic and extrinsic nature of compositional materials respectively, my experience with the production of audio recordings inclines me to a more technologically-focused appreciation, one that conceives of materials and technology as amalgamated, and it is this particular type of appreciation that I will now discuss.

As mentioned earlier in this chapter, Barthes' photograph is a spectator's photograph. He does not consider himself a photographer, nor does he discuss any experiences he may have had as an operator. This serves his purposes well; as he is primarily concerned with hermeneutic evaluation of the photograph, any recourse to the poietic processes of the operator are viewed as redundant to his interests. However, as I am unable to take such a stance with regard to the phonograph, I feel compelled to explore the technical processes, or at least what I imagine them to be, of certain recordings. To begin with, it should be noted that recording technology plays a significant role in the trace of the phonograph 
(as alluded to in chapter four). Though we may discuss the trace of a singer, saxophone or sonic environment in a phonograph, the trace is in fact wed to the technology that captured it, and reproduces it. In other words, the trace is not simply the remnants of a past reality, but of a past reality expressed through technology. By way of example, my recording of a ship negotiating the Gatun Locks of Panama Canal in 2006 is captured through two small diaphragm condenser microphones in an ORTF array, converted to digital at $96 \mathrm{kHz} / 24 \mathrm{bit}$ with a low gradient anti-aliasing filter. The trace level of this phonograph is not the spectre of the Gatan Locks, but these locks with the sonic signatures of recording technology embedded in them. With this particular recording, I am both the operator and the spectator of the phonograph, and as such, I am privy to a great deal more contextual information surrounding the technology used than anyone else. When I listen to phonographs of which I am not the author, I often (not always) imagine how the recording was made at a technical level. This is without question a kind of advenience for me, which can produce a completely distinct set technologically informed studium and punctum. I will now return to environmental sound composition to provide an example.

As I have noted throughout this thesis, Luc Ferrari's Presque Rien No. 1 is a landmark composition. It is considered such a work because the composer used vividly mimetic materials in its creation, within the context of a genre that actively sought to silence mimesis. Furthermore, it was very well received globally and helped to give credence to a nascent genre of experimental music. In my view, the impact of Ferrari's piece cannot be overstated. While I can appreciate the gravitas of such a work, it is not Presque Rien No. 1's status as a revolutionary work that provides me with a punctum. Nor does the content alone, understood here as the past reality of the Yugoslavian fishing village. These aspects belong to the studium. The aspect that pricks me is the sonic signatures of the recording system used: my $44.1 \mathrm{kHz} / 16 \mathrm{bit}$ version on compact disk reveals the combined noise floor of the analog recorder, microphones and preamps employed, the gentle rolling off high frequency content, and the subtle frequency modulation of tape across recording heads perhaps in need of some maintenance. The depth and clarity of the recording also prick me. It is the 
subtle harmonic saturation with clear fundamental tones that grabs my imagination, drawing me in to the work. An investigation into Ferrari's poietic processes reveals that he used a Nagra tape recorder in the creation of his early anecdotal works. These were the most highly regarded recorders of the era, used extensively by film sound engineers, musicologists and composers alike. It is likely that he used this machine in the creation of Presque Rien No 1. I do not require this information to experience this punctum, rather my curiosity is set in motion by it. I have never used a Nagra recorder, though I have used portable tape recorders in my own practice, and I can testify that the use of tape in the field delivers a kind of hue that I have not heard in digital recorders, devices that also impart their own hue. The hue of Presque Rien No.1 delivers a punctum. That is not to say all recordings made on a Nagra or indeed on tape will deliver such a punctum; it is the intermingling of the specific content with the technology, attributes made inseparable in the phonograph, that punctures.

Just as a recording system can provide a technological punctum, a playback system can offer a similar experience. In my personal studio I have a pair of Tannoy SRM-12B monitors powered by a Perreaux amplifier. The sound of this speaker/amplifier combination can produce a punctum when combined with certain materials. As mentioned, it is the intermingling of materials with the tape machine that produces the punctum I attribute Presque Rien No. 1. Likewise, when certain materials engage with my monitoring system, a technological punctum may be produced. That is not to say all materials played back through this system will produce a punctum. I do not experience this type of punctum when listening to Presque Rien No. 1 on this system. However, Sly and the Family Stone's album There's a Riot Going On (1971) wed to the Perreaux/Tannoy combination, always produces this punctum for me. The exact reason is difficult to pinpoint, but it is true to say that I do not experience the same punctum on other systems when playing this album, nor do I experience this punctum with every recording on this system. To my mind, this punctum is related to the performance of the Perreaux/Tannoy combination in relation to the materials it reproduces. 
It should be emphasised that the detection of the studium and punctum in every recording is dependent on playback technology: put simply, without playback, a listener cannot experience the materials of a recording. Indeed the quality of an audio playback system can greatly assist in the detection of certain puncta; the 'depth and clarity' I attribute Presque Rien No. 1 is an example of this. However, this should not be confused with the reproduction punctum. Though my Tannoy monitors emphasise this depth and clarity, I can hear this depth and clarity on other systems, on headphones, and even my internal laptop speakers (though to a lesser extent). I hear the depth and clarity of this work as born of the tape machine upon which it was recorded. This leads me to a very important point: though we are dependent on each aspect of the phonograph (production, storage and reproduction) for phonographic appreciation, that does not mean a listener cannot attend to and analyse the sonic signatures of these aspects independently from each other. It is a matter of attentional focus.

As mentioned earlier, Barthes would quite possibly relegate a technologically focused engagement with production of a phonograph to the studium, given that this trace is a result of poietic process and in many cases, the composer intends that the listener is aware of technology. Westerkamp's work, within the frame of acoustic ecology, is exemplary here. However, in my case, technological engagement forms a substantial part of my esthetic analysis of the phonograph, and as such my ear searches out the technological coupling with materials irrespective of composer intention; indeed technological coupling occurs as a matter of due course, regardless of whether a composer intends a listener should be aware of it or not. For me, technological listening can result in a punctum; and such listening is accommodated in Barthes' own analysis. Of the punctum, he concludes that 'whether or not it is triggered, it is an addition: it is what I add to the photograph and what is nonetheless already there' (1981, p. $55)$. 


\section{Punctum as blind field}

Barthes' suggestion that a punctum is born of esthetic analysis, yet something embedded at the trace level; that is, something 'already there', is significant. It ties spectator engagement to the 'brute presence' (Rancière, 2013, p. 87) of the referent, a point I alluded to in chapter five. Barthes expands this argument, introducing the concept of the blind field:

\footnotetext{
The cinema has a power which at first glance the Photograph does not have: the screen (as Bazin has remarked) is not a frame but a hideout; the man or woman who emerges from it continues living: a "blind field" constantly doubles our partial vision. (1981, p. 55-56)
}

At first glance, we might conclude that the 'blind field' of cinema, and indeed the 'deaf field' of the phonograph, is a condition born of temporal progression in mediatised events, and that photography would fail to produce a blind field on the basis that the photograph is without temporal progression. However, Barthes suggests that a blind field is born when a punctum is detected. He detects such a field in James Van der Zee's "Family Portrait" (1926):

\footnotetext{
When we define the Photograph as a motionless image, this does not mean only that the figures it represents do not move; it means that they do not emerge, do not leave: they are anesthetized and fastened down, like butterflies. Yet once there is a punctum, a blind field is created (is divined): on account of her necklace, the black woman in her Sunday best has had, for me, a whole life external to her portrait. (1981, p. 57)
}

I do not doubt that such a condition exists. Though we are ceaselessly drawn to the pose of the photograph, where a punctum is detected our imagination takes over to some degree. However, as Barthes suggests, our imagination is still tied to the photograph itself. However fanciful we imagine this blind field to be, it is not an alien field; that is, our imagination is always tied to 'what nonetheless is already there' (1981, p. 55), the brute presence of the referent always returns our imagination to the 'that has been' of the photograph. This is where the photograph and phonograph differ. Though when listening to a phonograph, an 
individual may 'divine' a second field related to the source materials, they can also divine other experiences that find no foundation in the materials themselves. This is perhaps its most extraordinary feature; a feature that I will now turn to.

\section{Punctum as memory}

Barry Truax' Riverrun (1986) produces a punctum for me, though this is largely because I do not detect his ecological concerns in this work. This punctum is of a very common variety in phonography, yet entirely absent from photography: the punctum that recalls a certain person, time, or event in our lives. Importantly, this person, time or event is not captured in the phonograph itself, rather the phonograph compels us to engage with our memories of that person or event. In short, where this punctum is concerned, the sounds embedded in the phonograph reference a specific past reality (whatever that may be), yet we are engaged with another. In Riverrun I am transported to the $9^{\text {th }}$ floor of the Victoria University Library some 17 years ago, where I analysed this piece for a 200-level electroacoustic composition paper. I sat there, with a classmate, and we discussed this work having listened to it together. Upon hearing this work today, I immediately consider my classmate, a close friend at the time, though someone I have not seen nor thought about much since that time.

This experience appears to be widely appreciated. Hans Baumgartner's "Remembrance of Things Past: Autobiographical Memory and Emotion" (1992), presents his research into such a phenomenon. Baumgartner asked 73 students if they could think of a piece of music that reminded them vividly of a person or past event. Only 3 students (2.19\%) identified that they could not:

The results of this study suggest that the subjects who participated in this research had experienced a situation in which a piece of music had become associated with an event from their lives so that hearing the piece of music evoked memories of the original episode. Most of the instances of this phenomenon reported by undergraduate marketing majors involved relationships with past or present lovers or experiences with family and 
friends, and although some people described unpleasant episodes, overall there was a significant bias toward remembering happy events. Most personal experiences for which the phenomenon occurred were strongly affectively charged, and the recollections triggered by the music were described as vivid and emotional and as involving a reliving of, and being accompanied by imagery descriptive of, the original episode. (Baumgartner, 1992)

In Music in Everyday Life (2000), Tia DeNora recounts the experiences of Lucy, a woman who regularly listens to some of the Schubert Impromptus as a way to 'foster a sense of inner calm':

First, for Lucy, the works are associated with comfort; they are bound up with a complex of childhood memories and associations. Her late father, to whom she was very close, used to play the piano after dinner and these works, wafting up the stairs, were ones Lucy used to hear as she was falling asleep. (DeNora, 2000, p. 42)

Lucy exhibits the kind of associative listening phenomenon that Baumgartner describes. As noted earlier, in Sonic Experience, a Guide to Everyday Sounds (2006), Augoyard and Torgue term this experience as anamnesis. Anamnesis is described as:

an effect of reminiscence in which a past situation or atmosphere is brought back to the listener's consciousness, provoked by a particular signal or sonic context. Anamnesis, a semiotic effect, is the often involuntary revival of memory caused by listening and the evocative power of sounds. (2006, p. 21)

Anamnesis may also be detected through olfactory experience; that is, smell also has an ability to involuntarily evoke memories and associations. Though the mediatisation of olfactory experience seems a long way from the level of sophistication found with aural and visual mediatisation, it is important to note that smell holds an anemnetic ability. ${ }^{74}$ Where sound is concerned, it should be noted that anamnesis is a feature of sonic experience in general terms, whether

74 Trygg Engen provides an interesting account olfactory experience in Odor Sensation and Memory (1991). 
experienced through the phonograph or via a sonic trigger experienced in situ. As such, both a recording of a song, or the sound of a train horn may trigger anamnesis. Augoyard and Torgue do not make a distinction between situated anamnesis and anamnesis experienced through the phonograph, their examples are wide ranging and include film soundtracks and recorded songs to the sounds of machinery and fun parks, but I believe such a distinction is worth making. Anamnesis in phonography is of a particular variety, one that differs significantly to that experienced in situ.

As Mark Katz notes in Capturing Sound (2004) that a phonograph may be replayed is one of its core features. Though no two copies, digital or otherwise, can ever be the same from a phenomenological perspective, I believe we are able to determine that two recordings have the same source. For example, though my vinyl copy of The Flamingos' 1959 recording of Warren and Dubin's "I only Have Eyes For You" is different from my digital version (presumably transferred from tape), through the sonic signatures of the respective technologies, I can detect that the source material is ostensibly 'the same'; that is, my ear can focus on the phonograph at the initial stage of production - a captured mix on tape - while ignoring the signatures of the storage format and the final stage of transduction. I believe that this ability is widespread, or perhaps it is better to say that the storage format and final stages of transduction are not widely considered. Either way, I am able to determine the Flamingo's version regardless of the delivery format, and I suggest that the vast majority of listeners familiar with the recording do also. This is not to say that the sonic signatures of recording formats are unimportant where anamnesis is concerned; indeed a particular version of a record may produce anamnesis where others do not, the point I am trying make clear is that though the delivery format may change (even radically), a listener is able to detect that one version is essentially a repeat of a previously heard version. It is the repeatability of the phonograph that gives us an ability not afforded in situated experience. Repeated listens to (largely) the same movement of air creates an indelible code in our memory. If we begin to associate a particular person or event with a piece of music, our access to that person or event is all the more vivid, on the basis 
that our connection to the past is temporally collapsed in the phonograph: it is the same recording we hear now as we heard then. With this in mind, we might conclude that though Lucy's recording of Impromptus produces an anamnesis for her, it would be a far more vivid anamnesis if she had listened to this particular recording with her father, and more vivid still, if her father had been the performer in the recording.

For me, The Flamingo's version of “I Only Have Eyes For You” produces an anamnesis, a punctum. When I hear this recording, I am immediately and involuntarily reminded of my parent's record player, the wooden cabinet it sat in, and ultimately, the experience of playing my parents records as a child. This recording has other puncta for me, of a technological variety, but I am chiefly 'punctured' by this experience; pulling records from their sleeves and setting them on the platter, needle to the groove. A deeper affecting power of this anamnesis is a kind of discovery: through their taste in recordings, as a child I discovered a part of my parents that defined them as individuals, with interests other than parenting. The punctum here is the realisation of a child who, through his parent's records, discovers that his parents are not only his parents. I rediscover this fact upon every replay of the song. When I whistle this song, or hear another version of it, I do not experience the same punctum. This punctum is tied to this recording alone, not just experienced upon hearing the original vinyl record my parents played, but any and all reproductions of it. It is born of its very specific features. I am not only familiar with these features, but with countless listens over the years, every nuance of this recording including the sonic signatures of its initial production, has become ingrained in my memory; my memory is not just familiar with this recording, it is wed to it.

Where environmental sound is concerned, there can be little doubt that it holds the power to connect a listener to past experiences in a process of anamnesis. By way of example, Peter Cusack's Dawn Chorus, Chernobyl Town (2012), as discussed in chapter three, is a recording of birds at dawn in a desolate environment. When I hear it, I am reminded of the many dawn choruses I have experienced while travelling. The bird life is foreign to me - I am unable to 
identify any of the birds I hear - and it is this foreignness that provides a punctum. Having recorded many dawn choruses myself, I am drawn, in a process of anamnesis, to prior experiences of recording such environments; the mixed feelings of being away from home, the feeling of privilege at being up so early to experience something that others are not, the excitement of recording something new and the feeling of potential that such recordings offer. Though my particular anamnesis is one related to field recording, others may experience a less technologically-focused variety. I think it likely that anyone who has experienced a dawn chorus in a foreign country will engage with their own experiences of such moments. Cusack, and people familiar with the Ukrainian wildlife, may have a more specific anamnetic experience when listening to Dawn Chorus, Chernobyl Town.

We may even consider acoustic ecology to be greatly informed by the phenomenon of environmental anamnesis, whereby listeners are conceived as connected to past events or people, or concepts such as community, family or civic duty through sonic triggers. In other words, the power of environmental sound to reference the non-auditory is the basis upon which the arguments of acoustic ecology are mounted, though as I have asserted in chapter four, acoustic ecologists take this relationship a step further, tacitly presuming to know the kind of experiences these acoustic triggers will produce in listeners.

Augoyard and Torgue appear to support an inter-subjective reading of anamnesis:

Although it is essentially subjective, anamnesis also has an archetypal dimension. Specific sounds can produce common references for a given culture: sounds of flowing water, rain, crackling fire, thunder, and singing birds, but also sounds of industrial automatic devices, cars, and urban drones. There are many shared backgrounds over which individual perceptions are laid. Archetypal anamneses are less conscious than others, but they can be just as effective. (2006, p. 23) 
I refute the notion of inter-subjective anamnetic experience. While it is conceivable that individuals may encounter similar types of memories due to shared cultural norms and histories (as Augoyard and Torgue suggest), the authors effectively disregard the specificity of an individual's memories and their unique intentional perspective in the anamnetic experience. From a phenomenological perspective, the supposed 'archetypal dimension' of anamnesis does not hold much value. Furthermore, I do not believe that one person can access another's anamnetic experience, which I believe to be the fundamental misstep of soundscape theory.

Though I am convinced of the power of environmental sound to create anamnesis in listeners, I am yet to encounter a sound in situ that draws me to a specific time, place or person with the accuracy of recorded sound. This may be due in part to the fact that situated sound can never be repeated with the same level of accuracy as recorded sound, but I also believe that the multi-modality of situated experience confounds our ability to attend to the specific in the same way mono-modal experience does, as our attentional focus is distributed between our other senses. We might recall Ingold's assertion that:

\footnotetext{
The environment that we experience, know and move around in is not sliced up along the lines of the sensory pathways by which we enter into it... in ordinary perceptual practice these registers cooperate so closely, and with such overlap of function, that their respective contributions are impossible to tease apart. (Ingold, 2007, p. 10)
}

Where environmental sound composition is concerned, it might be argued that the primary form of anamnesis encountered is closer to that of situated anamnesis, certainly upon first listen. By way of example, if I were to hear the Eastbourne fire station's 1pm siren (a soundmark of my home village, every Saturday, for as long as I can remember), in an environmental sound composition, I would no doubt encounter the less specific form of anamnesis I associate with the situated experience. However, on repeated listens to the specific composition, further, more specific anamnesis may arise, those related to the phonograph and perhaps events, people or circumstances related to my 
current context, rather than the anamnesis produced by hearing the siren itself. In this way, anamnesis in environmental sound composition has an ability to become multifaceted with some ease. Truax' Riverrun provides an excellent example of this. When I first heard Riverrun, the sounds of running water may have provoked anamnesis (I do not recall this happening, though it is entirely possible). These days, my anamnesis is related specifically to my classmate. The question of whether one can experience multiple anamnesis in a single instance is worth further research, but for now I will note that, at the very least, this punctum has the power to change, and that, where environmental sound composition is concerned, the movement from anamnesis associated with situated listening to a phonograph-specific anamnesis appears to be an easy one to make. It could also be argued that the ability for environmental sound composition to encourage anamnesis akin to that of situated listening is indeed its tacitly acknowledged primary power: environmental sound has a pronounced capacity to engage listeners with memory on first listen.

The very notion of anamnesis expands the notion of temporality in the phonograph from one conceived as a duality between the past reality of the event and the present reality of audition, and provides a third dimension, measured at the distance between the present audition and the moment our memory is involuntarily taken to. In this way, where anamnesis is at play, we can talk about the present reality of audition, the past reality of the content, and the very personal reality of our memories. Such a punctum is not a feature of photography. As Barthes has it, 'the Photograph is violent: not because it shows violent things, but because on each occasion it fills the sight by force, and because in it nothing can be refused or transformed' (1980, p. 91). The continued pervasiveness of the spectrum, its 'brute presence' as Rancière has it, is identified by Barthes as the Photograph's noeme. It is this very feature that precludes a kind of photographic anamnesis:

Not only is the Photograph never, in essence, a memory... but it actually blocks memory, quickly becoming counter memory. One day, some friends were talking about their childhood memories; they had any number; but I, 
who had just been looking at my old photographs, had none left. (1981, p.

91)

With this in mind, we can conclude that the phonograph gives us a unique blend of experiences that simply do not exist for other kinds of mediatised reality. Firstly, the phonograph resounds and engages with the present through a pairing with the acoustic space and the intentional listener. It is a spatially and temporally compelling presence, one in which the ear scans along the temporal span with varied attentional focus in the ongoing analysis of the auditory scene. As discussed in chapter five, the cognitive processes of the listener and the physical reality of sound waves moving air attunes the listener to the present in a manner not observed in photography or film. Secondly, like the photograph, the phonograph attests to a past reality. Barthes concludes that 'the noeme of Photography is simple, banal: no depth: 'that has been' (1981, p. 115). We too may conclude that the phonograph declares 'that has passed', a testament to its documentary power, but it is not this aspect that lies at the heart of the phonograph's noeme, though it is an aspect of it. In truth, the past is ceaselessly pulled into the present with a vigor that eludes photography, so while we are aware of the past reality, our ability to perceive the past is confounded by our keen attentional focus in and to the present. Nevertheless, the past is most certainly embodied in the phonograph. The third experience that is unique to the phonograph is its ability to compel a listener to a referent completely independent of the phonograph itself, an anamnesis, a punctum. This is truly a unique feature of the phonograph with no equivalent in visual media, for our sight is violently 'filled by force' in photography and, perhaps with even greater force, cinema (especially sound cinema), revealing nothing but the referent, or 'blind fields' tied to the referent. Yet as the phonographic referent too fills our ears by force, we are at times simultaneously forced to recall another referent altogether; anamnesis is a powerful force, one over which we have no control, and one that appears to have at least as much power as the past reality itself. Finally, though we may too experience anamnesis in situ, the phonograph offers a unique form of this, born of its ability to be repeated, providing a level of specificity to anamnetic experience that aural experience in situ cannot. 
Though we may conceive of these core aspects of the phonograph discretely, it is the intermingling of them that produces the thoroughly unique experience of the phonograph, in which a rich and changing dialogue between the mediatised referent, the present audition and the experiences of the listener creates an object so heavily invested in the noetic processes of the listener that defining the phonograph as an noematic object has thus far proven elusive. It is my belief that in allowing the phonograph to be viewed in the terms laid out in this thesis, that genres of phonographic works, especially those of environmental sound composition, may be allowed to blossom in new directions. 


\section{Volume II}

\section{Portfolio of Compositions}

\section{Introduction}

Before detailing the compositions of this portfolio, I think it is important to place them within the broader context of environmental sound art. As noted in Volume I my pieces are primarily concerned with the phonograph, and it is the interaction of the environment with acousmatic art that constitutes the frame within which these works were created. It should be noted that there is a long history of phonograph-specific environmental sound works in Australasia, and it is in this context that my works also reside. New Zealanders Douglas Lilburn, Jack Body, Susan Frykberg, John Cousins, Chris Cree Brown, Annea Lockwood and Reuben Derrick, along with Australians Garth Paine, Andrew Skeoch, David Lumsdaine, Ros Bandt and Leah Barclay, are just some of the composers who have worked with environmental sound and the phonograph. I myself have a particular affinity with the work of Douglas Lilburn, as his work involves a particular intersection of technology and environmental sound that I find compelling. I will show how his work has influenced my own in the detailing of my pieces that follows shortly.

Jose Iges (2000), in his paper "Soundscapes: A Historical Approach", writes:

Three very different tendencies have emerged within soundscape in general. The first... was represented by the more or less direct adherents of Murray Schafer. The second involved what we could call freer work with the sound environment, in some cases incorporating elements of poetry, documentary or reportage, and in others creating "sound bridges" between two natural or urban environments, relating them directly or with the help of telephone lines or communications satellites... The third tendency has been represented in recent years by the work of sound artists like the Spaniard Francisco López. In his case, the acoustic environment is considered to all 
effects as the most complete "synthesiser", providing rich, diverse raw material for his work (2000).

Though Iges considers these three categories as subgenres as 'soundscape' (Lopez would undoubtedly refute this notion, given his views on "environmental sound matter" [1997]), I feel his appraisal is accurate if we consider the three categories as subgenres of environmental sound art. In one category we have soundscape composition and environmental sound compositions that seek to promote the documentary feature of the phonograph ahead of its abstractive feature. In this category, the phonograph plays a central role. Likewise, in the third category we find works tied to the phonograph, though in this instance, the abstractive quality is paramount. As Iges writes, in his middle category we find "freer work", which I take to mean works that are not bound to the phonograph or the concept of acousmatic art. We might trace the origins of this category from the early environmental listening practices of Luigi Russolo, the situated listening practices of John Cage, the sound installations of Max Neuhaus, Bill Fontana, and indeed contemporary practices including Florian Hollerweger's real-time situated processing works. In the context of Australasia, we find the sound installations and radiophonic works of Ros Bandt and Leah Barclay, along with the environmental sound installations of Sam Hamilton. In many respects, this second category represents an area of environmental sound art that has grown the most, perhaps due to its ability to engage with other arts practices, often with audiences appreciating the works collectively and in real time. It may also have grown, in part, due to the lack of problems associated with the combination of environmental sound and acousmatic art as outlined in Volume I. Indeed, as Iges notes, this is considered a category comprised of "freer works"; presumably they are "freer" than category one and three works as they are not tied to fixed media representation.

In my opinion, this second category of freer works, while very important to environmental sound art, has less to offer the discussion of environmental sound composition, and by that I mean acousmatic compositions dependent on the phonograph for appreciation. It is the division between the recording and its 
replay - a temporal and spatial division - and the relationship with environmental materials to the listener in this divide that defines my field of research. With this in mind, I suggest that we might simplify Iges' delineation of environmental sound art, by considering just two categories of works: phonographic works, and non-phonographic works. In the context of this thesis, I am solely concerned with phonographic works, as I seek to contribute to discussions surrounding the phonograph in environmental sound art. It is my hope that in reassessing these roles, that phonographic works might attain the level of freedom Iges detects in non-phonographic works of environmental sound art.

Before describing my compositions in detail, it is important to note that some of my works include practices that involve category two type installations; specifically, site-specific and temporary installations, live improvisation and realtime processing. To be clear, the compositions in this portfolio are acousmatic compositions, not documents of live performances (where such performances are recorded). Though I may wish to exploit the documentary aspect of the phonograph in some cases, audiences may not hear this feature, and overtime, the abstractive feature of the works, promoted not least of all through the repeatability of the phonograph, may become the dominant reading. Regardless of the specificity of listener responses, what is guaranteed is that a listener will experience a phonographic work in each instance. In other words, though a listener's esthetic evaluation of the work may change over time and indeed upon every listen, the phonograph becomes the frame through which all understandings are formulated, hence its importance.

Finally, I would like to draw attention to what may seem like a paradox within this thesis. In Volume I, I argue that the dramaturgical information that often accompanies environmental sound compositions is the very thing that can hinder a fulsome response from listeners, yet what follows here in Volume II constitutes the kind of dramaturgical information I argue against. However, I think it is important to note that in the context of this thesis such information does not serve to orientate the listener towards my poietic concerns as a means 
to guarantee the success of the works themselves; my desire for listeners to engage with these pieces on their own terms far outweighs my desire for them to understand my poietic processes. Rather, the information that follows serves simply serves simply to make the relationship between my practical research and my theoretical research explicit. As such, the inclusion of dramaturgical information in this thesis is designed to demonstrate how the technical and conceptual methodology of my compositions actively explore the notions of the composer, the sites of production, the role of the phonograph, while probing the various temporal relationships between the materials, the composer and the listener. In particular, I describe the way in which particular methodologies may elicit new kinds of listener responses, without dictating what those responses might be.

\section{Methodology}

The compositions that comprise this second volume were created at various times over a four - year period, between 2011 and 2015. As discussed in Volume I, it is often the case that environmental sound compositions, where fixed media phonographic works are concerned, follow a methodology that involves the recording of an environment first, followed by manipulation and arrangement at a later time in the studio. At the beginning of my research, I devised a compositional methodology that sought to combine the site of recording and the site of production into one time and place. I did this by bringing studio production equipment (loudspeakers, computers, microphones, MIDI controllers etc.), into the environments I was recording, and I would improvise with these tools in situ. The subsequent single-take recordings of these events might be considered documents of such improvisations, but as I have already alluded to, they are much more than this. This methodology was used in the creation of more than half the works presented in this portfolio.

Other pieces follow a more traditional path (site recording first, production later), while others are more closely aligned with the genre of phonography, in which the recordings are largely untransformed. In both instances, my pieces 
explore the established methodologies of such practices, while looking to highlight some of the concerns as laid out in Volume I. A final piece, the first in this portfolio, is a complex hybrid work of methodologies. It involves real time improvisation in situ, and the secondary site of studio production. It employs both ambisonic and stereo diffusion. For each work I will detail the methodology and compositional concerns in written format, followed by a visual representation of the sites and my intervention, along with a brief technical description of the equipment used. I have included a key at the beginning of my portfolio that describes the equipment used.

As far as the ordering of works is concerned, it is desired that a listener follows the order I have prescribed, as there is a certain rationale I have followed in the programming. The first track is the most complex while the last track is perhaps the most simple. However, in following the ordering from first to last, a listener might be led to hear the final track in less simplistic terms, and by this I mean with an ear to the complexities of phonographic framing. In other words, it is the development of ideas over the course of this portfolio that leads to a particular listening perspective, and while I do not presuppose what this perspective might be for the listener, I do believe that in following the particular order I have prescribed, a listener's perspective will change throughout the portfolio in a manner that is more coherent than a random audition of tracks would allow.

Finally, I have included the individual tracks in digital format so that a listener may import the files into their preferred digital audio workstation, and set up the surround diffusion as they prefer. However, I have also provided a Reaper session file (with audio), set up for octophonic diffusion. For those interested in using this format, please follow the instructions below:

1) Download and install Reaper from http://www.reaper.fm/download.php

2) Download the Tetra Mic plugin VVMicVST from http://www.vvaudio.com/downloads and place in the appropriate folder, as specified by the prompter (this depends on your operating system). 
3) Make sure the octophonic array is set up correctly (audio interface output 1 - 8 connects to speakers 1 - 8). It does not matter if the array is set in pairs or in a ring format, but make sure that the VVMicVST plugin decodes to the speakers in the format you select. For example, you will need to use these azimuth settings for the following octagon configuration (Fig. 2):

Mic 1: 22

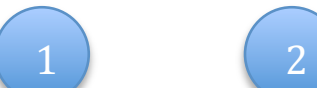

Mic 2: -22

Mic 3: - 67

8

Mic 4: -112

Mic 5: -157

Mic 6: 157

Mic 7: 112

Mic 8: 67

Figure 2: Octagon Diffusion Format

4) On the DVD provided, navigate to REAPER SESSION $>$ PORTFOLIO $>$ and open the Reaper session file named - VOYCE PORTFOLIO LISTENING SESSION_1.RPP - then push play when your VVMicVST is correctly set. 


\section{Figures Used in This Portfolio}

In the description of my compositions, I employ visual images to assist the reader in gaining an appreciation of where the recordings were made, and how audio technologies were used. I employ maps and photographs specifically. The maps used show the wider terrain, and the specific location where the recordings were made. The photographs used are taken from the site of recording, and I have superimposed icons over them. These icons represent the technologies used in the creation of recordings. The icons below will appear in visual diagrams that follow written descriptions of each piece in the portfolio. As many of these icons appear in multiple figures, it is useful to introduce them here so that the reader may become familiar with what they represent.
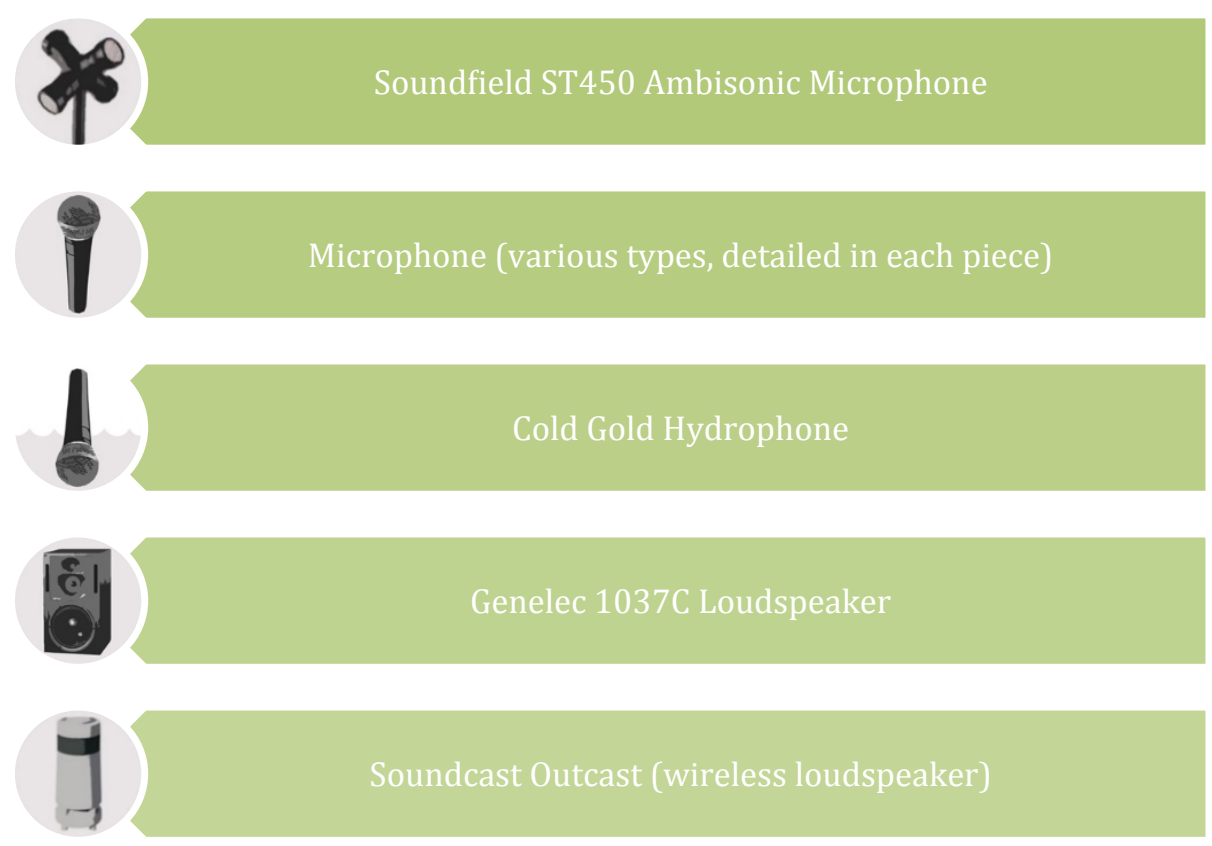

\section{$\cos$}

Sony WM-D6C Cassette Recorder
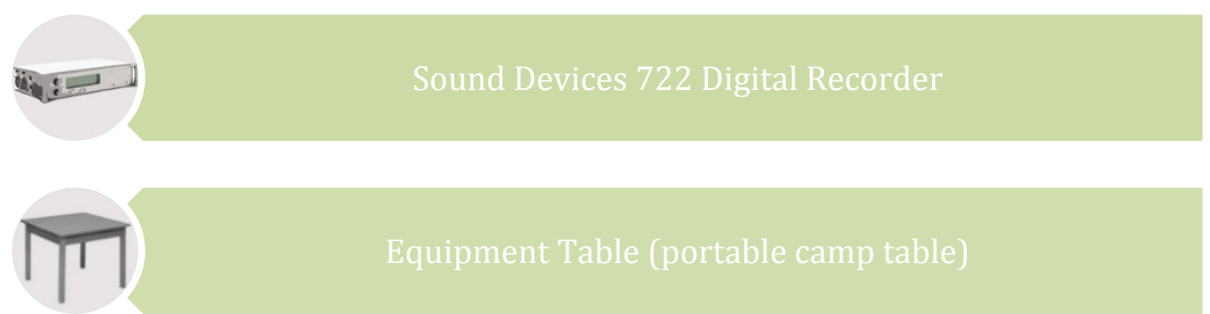

Equipment Table (portable camp table)

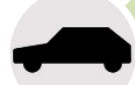

Daihastsu Sirion GS (2003)

Figure 3: Key to Portfolio Icons - rendered by Jina Yoon 


\section{Portfolio of Compositions}

\section{TRACK 1}

Title:

BFMT + 2-CHPHNO-001 (2015)

Duration:

$12^{\prime} 06 "$

Format:

B-format and 2-channel phonograph with improvisation on site and post-production

Location: Various

This piece was created around a single 2-channel improvisation made in a car, in a car wash. Using the Synth-In-A-Case VSTi (a software emulation of the EMS Synthi AKS by sound designer ZooTook) with playback through my car stereo, I improvised with the Synthi in counterpoint with the car wash as it cleaned the exterior of my car. I recorded this improvisation with the Soundfield ST450 microphone, which was placed in the middle of the car between the front and rear seats at head height. I also recorded the dry audio from the Synthi, that is, the output of the VSTi before it was sent to loud speakers. This dry recording formed the basis of my next series of recordings.

I then took my car to various locations around Wellington and Lower Hutt cities, including parking buildings, marinas, and windy suburban streets. I would lower the windows of the car (sometimes by only an inch or so) so that the environmental sound would drift into the vehicle. I then played the dry recording of my Synthi improvisation through the car stereo at exactly the same volume as the original improvisation in the car wash. I recorded each of these instances with the Soundfield ST450 microphone, itself placed in the same position as the initial car wash recording.

Next, I took the dry Synthi recording and played it back through portable loudspeakers (two 'Outcast' speakers made by Soundcast), in various environments, including quiet walkways in bush settings, at the beach, and in an 
art gallery. I again used the Soundfield ST450 microphone to capture these events. I measured the decibel levels at the Soundfield ST450 so that they were similar to those in the car. The reason for this (and the matching of playback volumes within the car) was so that in each recording, the level of the Synthi improvisation would be the same relative to the dynamic range (and noise floor) of the recording system. Finally, I took all these ambisonic recordings - the original improvisation in the car wash, the subsequent replays of it in the car, and the replays of it in other environments - into the studio and began to structure this piece. My main objective was to explore the idea of environmental space and situated listening space, and to do this I decided to structure the piece into three sections. In each section the original improvisation plays in its entirety.

\section{Recording Locations (Wellington Region, New Zealand):}

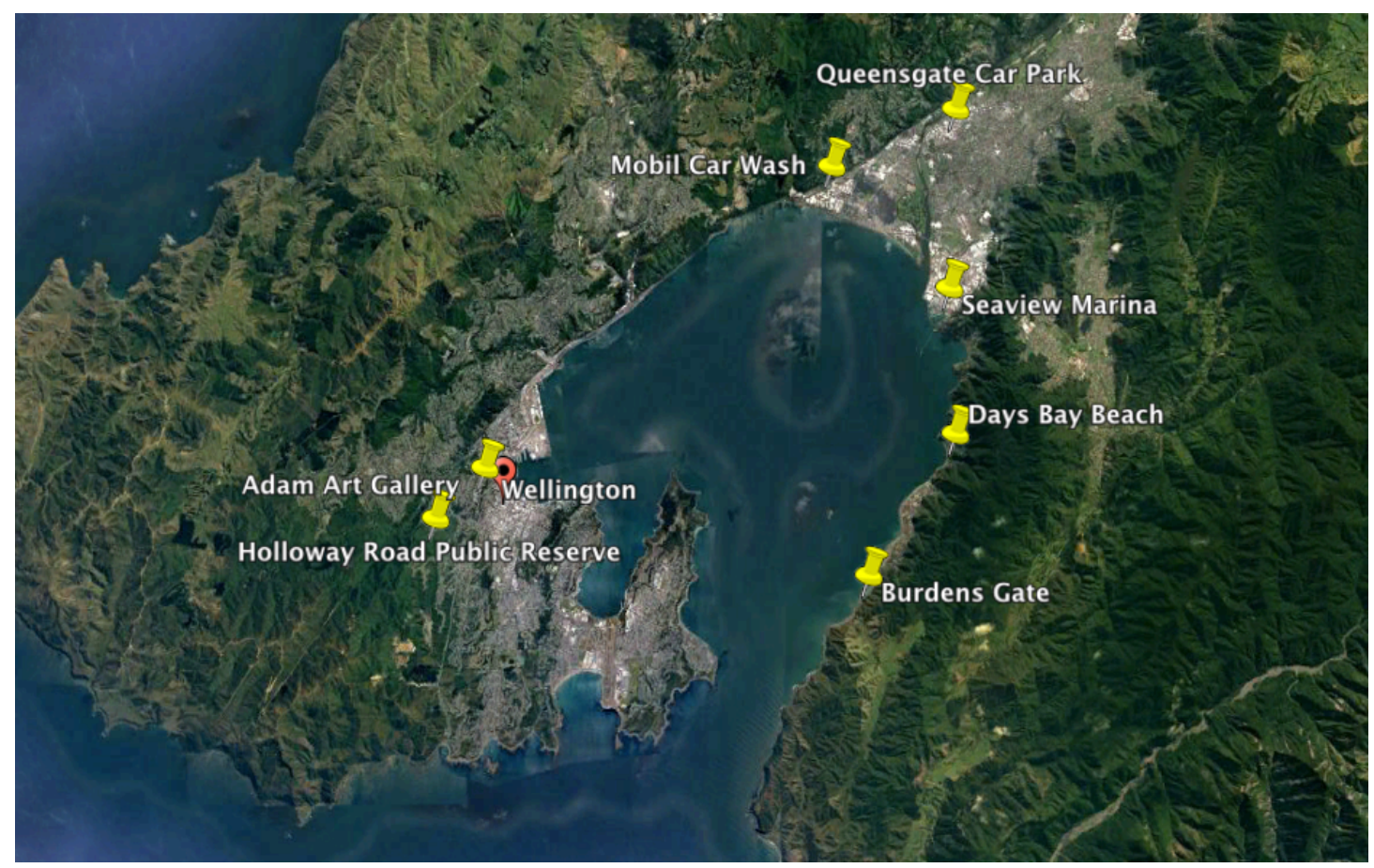

Figure 4: Google, Digital Globe 2016.

\section{Section 1:}

In this section, I explore the notion of 'nested space' (Smalley, 2007), that is, a space within a space. In this instance, the nested space (the car) is consistent 
between recordings while the wider space (the external environment) changes. In order to achieve this, I set one car recording in motion (made at a marina), and then I spliced it together with another car recording (made on a windy street with the windows open a fraction). As such, when the first recording plays, we hear the Synthi recording through the car stereo and the sound of the environment outside the car. When this recording cuts to the next, the Synthi recording and the nested space remain the same (due to the matching of $d B$ levels). The only perceptible difference is that the exterior space of the car is now a windy street, or with the next splice, a parking building. This section ends with the original recording: the Synthi improvisation in a car wash.

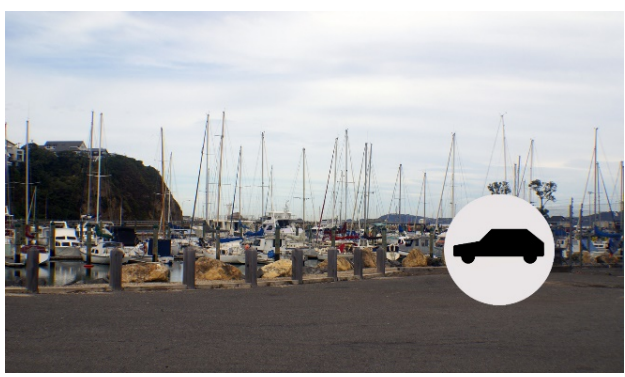

Seaview Marina (Seaview, Lower Hutt City)

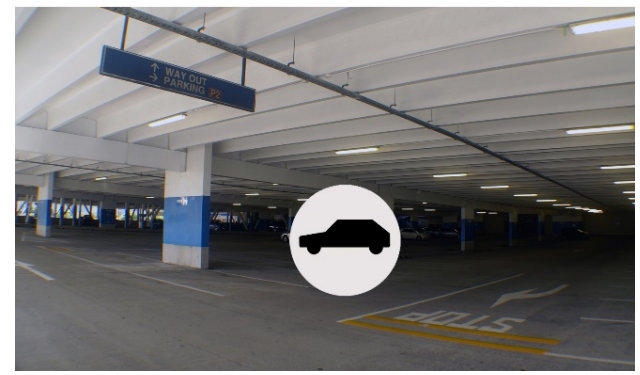

Queensgate Car Park (Lower Hutt, Lower Hutt City)

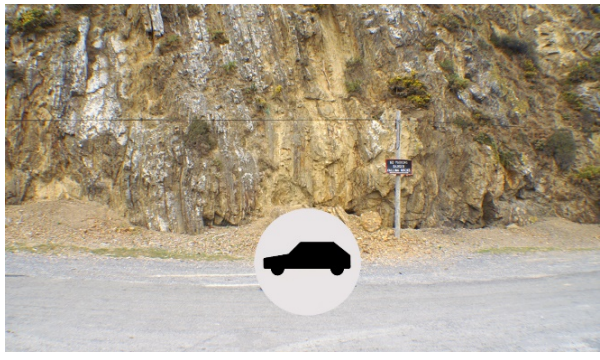

Burdens Gate (Eastbourne, Lower Hutt City)

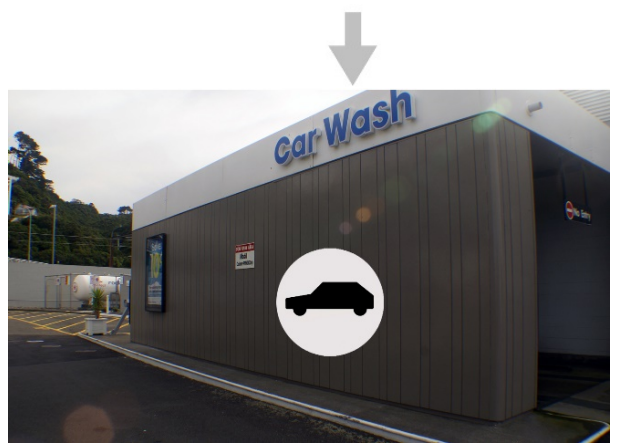

Mobil Car Wash (Petone, Lower Hutt City)

Figure 5: Section 1 Locations - Photographs: Thomas Voyce; Icons and Layout: Jina Yoon 


\section{Section 2:}

This section explores environmental space. The nested space of the car is no longer present: the recording of the Synthi improvisation is now played in the environment, and the result is that the recording is now part of the wider environment. Much like the first section, various environments are spliced together, with the matched $\mathrm{dB}$ levels of the Synthi recording allowing for comparable levels between recordings, though unlike the first section, crossfades are used to make the transitions smoother. This section begins with a quiet walkway by a stream, which then moves to a beach setting, which then moves to the reverberant interior of an art gallery. In each transition, the environmental space changes quite significantly, while the Synthi recording remains consistent. This section concludes with the last notes of the improvisation reflecting around the gallery space, then fading into the ambient noise floor.

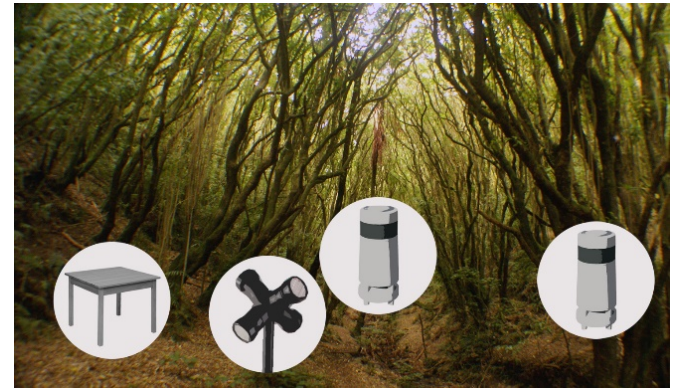

Holloway Road Public Reserve (Te Aro, Wellington)

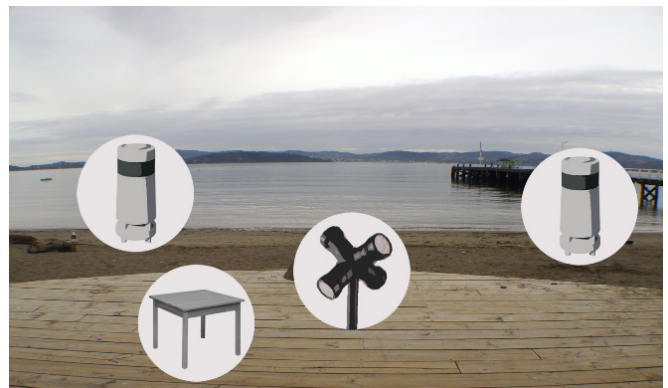

Days Bay Beach (Eastbourne, Lower Hutt City)

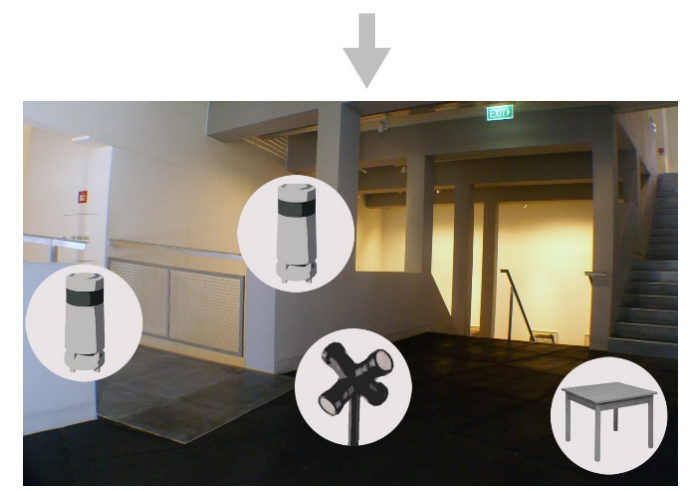

Adam Art Gallery (Te Aro, Wellington)

Figure 6: Section 2 Locations - Photographs: Thomas Voyce; Icons and Layout: Jina Yoon 


\section{Section 3:}

This final section is a replay of the dry Synth-In-A-Case improvisation in 2channel format without any environmental or surround sound component. From the front 2-channels of the listener's diffusion set up (channels 1 and 2) the recorded improvisation plays in its entirety. With no environmental space or surround diffusion to accompany this recording, the listener may be made aware of their current listening environment: the spatial transition from the car, to the environment(s) to the listening room is thus made possible by the consistent level of the improvisation throughout. In this way, it is hoped that the Synth-InA-Case holds the listener's hand in what is, in essence, a piece that deals primarily with environmental space.

It should be noted that the sound of the EMS Synthi holds significance for me in this piece and others in this portfolio. Douglas Lilburn, founder of New Zealand's first experimental electronic studio at Victoria University in 1966, used the EMS Synthi in a number of his works throughout his career. To my ears, the combination of this synthesiser and the sonic environments of New Zealand form a unique sonic signature within genre of electroacoustic music, and my use of the VSTi emulation and the current sonic environment of New Zealand looks to extend this signature into contemporary practices. My use of this combination is born not of a desire to appropriate Lilburn's work or methodology (my recordings are very different from Lilburn's on a number of levels); rather my use of the EMS Synthi emulation and the sonic environment recognises the fact that these trace elements have produced a punctum for me; one that is in part born of their historical usage in Lilburn's work, but also due to their raw materiality. 


\section{TRACK 2}

Title:

BFMTPHNO-001 (2015)

Duration:

6'07"

Format:

B-format phonograph with improvisation on site

Location:

Lowry Bay, Eastbourne

This piece might initially be understood as a single-take phonograph of a live improvisation. It is the first of five such recordings in this portfolio. This improvisation took place along a walking track in Francis Bell Reserve, Lowry Bay, Eastbourne. A Soundfield ST450 microphone was used to capture the performance. Four speakers were placed in a quadraphonic array in relation to it. My improvisation utilised four sine tones, each feeding one of the four speakers. A small amount of digital reverberation was used to enhance and extend the space: if a sine tone were mapped to the left front speaker, the remaining three speakers would carry the reverb signal. I mapped the amplitude and frequency of these tones to my Allen and Heath K2 controller. As such, I was able to manually fade in and out sine tones at various pitches.

The piece begins and a loud plane promptly emerges, passing overhead. After a slow glissando down, the plane tone arrives at a single low frequency, and then disappears from audibility. Before it begins to disappear, it is extended by the low sine tones I played into the environment. It should be noted that no additional EQ or processing has been added: the low tones of the plane were not that loud in situ, but the Soundfield microphone has responded with increased bass response, perhaps due to its height above the ground and/or capsule design. The sound of the plane is thus the impetus for the improvisation that follows. Taking cues from the birds of the reserve, I then play a series of notes that (loosely) attempt to mimic their songs. A relationship is established between my improvisation and the birds in a kind of call and response. This may be an imagined, one-sided relationship; there is no indication that the birds are in fact responding to my sounds though I may be responding to theirs. I then 
cease to improvise, allowing the environment to sound without my aural intervention. I imagine, with a degree of fantasy, that the birds have changed their songs in response to my improvisations. Regardless, the resultant phonograph presents a work in which a loud plane passes a sonic baton to me, which I in turn pass back to the environment.

An interesting feature of this phonograph is that it allows for the improvisation to be listened to repeatedly, and on repeated listens, I intentionalise a myriad of relationships that I did not experience in situ. As such, the phonograph renders the improvisation a concrete work. When I listen back to my improvisation, I am now able to hear formal qualities, born of the phonographic frame I have provided it with. Perhaps the most important feature of this work is that my role is different from that of many environmental sound composers. As discussed in chapter four, when environmental sound composers make overt manipulations in the studio, they may be perceived as creating a meta-document akin to the dub meta-document: they stand between the materials and the listener in an intermediate space acting as a kind of curator. This piece, however, is ontologically different, in that it is a single-take live recording, not a metadocument, and my intervention does not take place in the intermediate space of the studio, but in situ. If a listener is able to determine that the improvisation takes place in the environment (which I believe is entirely possible and aided greatly by the enhanced spatial dimensions of ambisonic recording), they might then begin to wonder about the role of the composer in such a work. They might then wonder about the function of the phonograph in this intervention.

It should be noted that the success of the work is not predicated on the listener's identification of the work's specific ontological features as rendered through the compositional methodology. In Barthesian terms, such identification is likely to belong to the studium of the work. My view, as detailed in Volume I, is that it is the generation of a punctum within a listener that determines a deeper engagement, an engagement that the composer has no control over. As such, from a poietic perspective, my goal in this work is to create an environmental sound composition with a unique ontology (which I have succeeded in doing), 
but the apprehension of this feature by the listener is not necessarily a pertinent esthetic outcome. It is my hope, however, that the poietic processes employed might lead to a unique experience for a listener; that is, the phonograph they encounter is unlike any they have heard before.

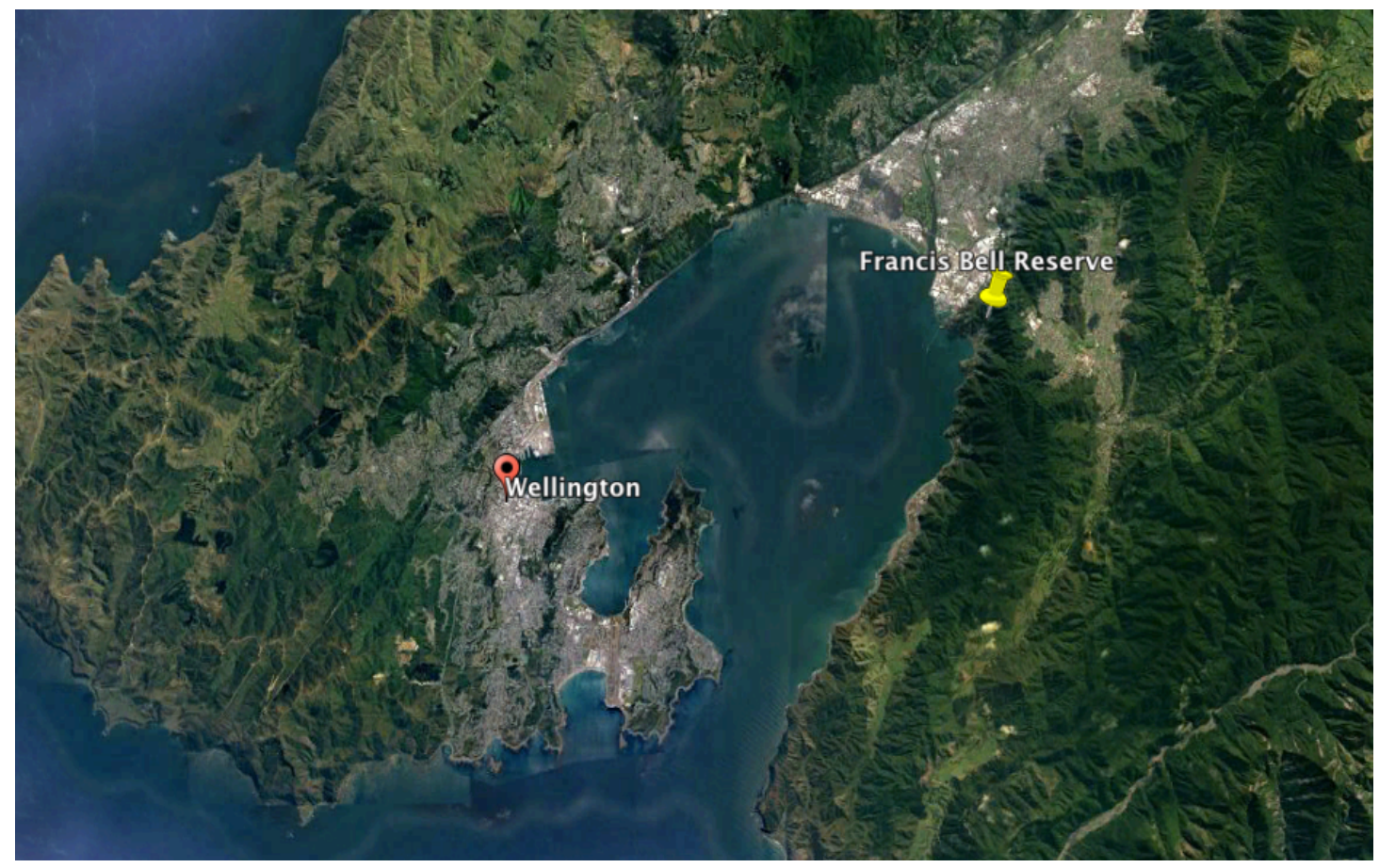

Figure 7: Google, Digital Globe 2016.

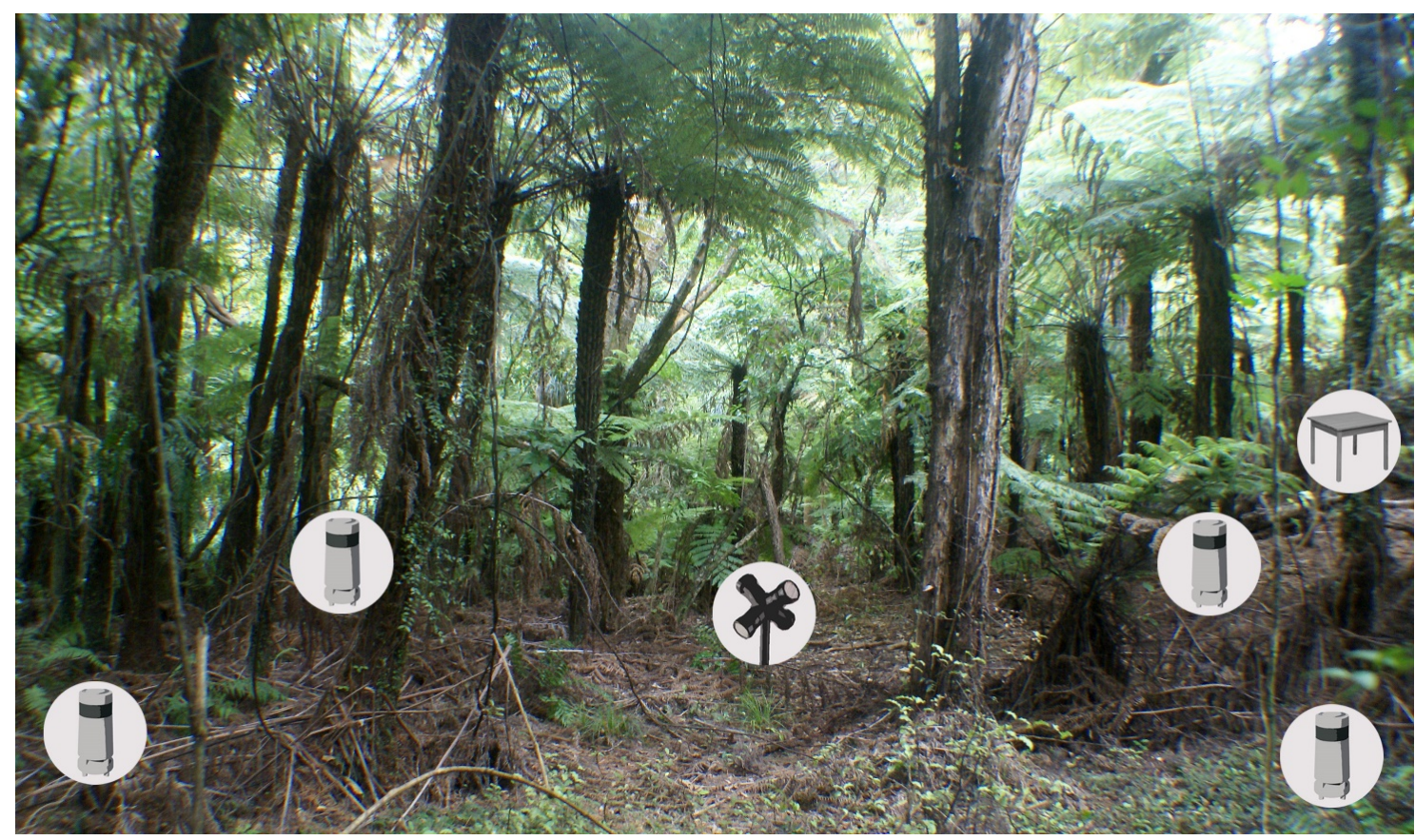

Figure 8: Francis Bell Reserve Configuration - Photograph: Thomas Voyce; Icons and Layout: Jina Yoon 
PARTIAL LIST OF EQUIPMENT ON TABLE (excluding cables, adaptors etc):

- Macbook Pro laptop powered by Hyper Juice external battery pack

- Ableton Live software (audio diffusion and B Format recording)

- Allen and Heath K2 Controller

- Metric Halo 8228 Audio Interface

SIGNAL PATH:

1) Recording

- ST450 microphone

- ST450 Preamp

- Metric Halo 8228 A/D converters

- Ableton Live recording tracks (W, X, Y, Z)

2) Diffusion

- Software: Ableton Live

- $4 \mathrm{x}$ sine tone oscillator

- Reverb (Live 8 Audio Effects "Dark Hall" preset with extended decay)

- MIDI control (K2 Controller)

- Discrete outputs (Metric Halo 8228 D/A channels 1-4)

- Wireless connection to $4 \mathrm{x}$ Soundcast Outcast speakers 


\section{TRACK 3}

Title:

BFMTPHNO-002 (2015)

Duration:

5'58"

\section{Format:}

B-format phonograph with improvisation on site

Location:

Adam Art Gallery, Victoria University, Wellington.

This piece is composed of a 4-channel improvisation captured in one take by a Soundfield ST450 microphone. This improvisation utilises four large Genelec 1037C studio monitors placed at various positions throughout Victoria University of Wellington's Adam Art Gallery. In this piece I elected to use the Synth-In-A-Case VSTi as the instrument to improvise with (two of them in this instance, in 2-channel pairs, thus the four speakers are fed by two stereo instruments). The improvisation was made at night with technical support from sound artists Andy Cummins and Chris Wratt. The gallery itself had few sounds in it at this time, and with the air conditioner turned off, the noise floor was very low.

My primary concern in this work is the engagement of aural architecture (Blesser and Salter, 2007). Though this environment is largely silent, when fed sounds through speakers the gallery speaks with a unique voice, born of its particular architecture, and it is this voice I wanted to engage with. The Synth-InA-Case has the benefit of being capable of producing very low sounds and very high sounds at significant amplitudes. This helps to engage the various resonances of the reverberant gallery in dramatic ways. Additionally, the gallery has a very unique spatial profile, born of its unique, multi-level, multi-gallery layout. I placed each of the four speakers on different levels, in different galleries in order to exploit this aspect. The Soundfield ST450 microphone was placed in a fairly central location, one that acts as an intermediate space between various parts of the gallery, thus no one speaker is in immediate proximity to the microphone. The floor beneath the microphone is made of rubber, and as such, this space is much less reflective than the other rooms, which use concrete and 
wooden flooring. This has the effect of helping make the individual rooms more directionally focused, as there are few additional reflections around the microphone to cloud directivity.

The improvisation was conceived in order to engage with the spectral and spatial qualities of the gallery, and therefore the performance utilises full spectrum audio (low and high sounds), wide dynamic range (quiet and loud sounds), and durational variation (short and long sounds). As I had not determined the exact acoustical properties of the art gallery before the recording, particularly the frequencies of room modes and decay times of reverberation, my improvisation seeks out these features, through the inclusion of low frequency to high frequency sweeps (albeit arpeggios as opposed to glissando - the usual acoustic test method), and short sounds with less pitch content to reveal the various decay profiles. As such, this piece is designed to impart, primarily, the architecture of the space using the sonic signatures of the Synth-In-A-Case.

Part way through the improvisation, I introduce the sound of a natural environment (re-pitched down an octave), using a recording I made in the hills behind Eastbourne. The use of this recording is to once again reference Douglas Lilburn by bringing the notions of the natural environment and technology into the work. I also wanted to create an unusual presence in the room, highlighting the notion that one environment is coupled to another through audio playback. In this instance, the result is a little unnerving, as the coupling of birds with a reverberant gallery space feels compelling, yet unnatural, partly because they are re-pitched, but also because birds are out of place in a reverberant space. The affect of this reminds me of Schafer's schizophonia, and though I do not fundamentally agree with his concept (see chapter four), I also feel that the inclusion of bird recordings might demonstrate the 'nervous' condition he refers to. 


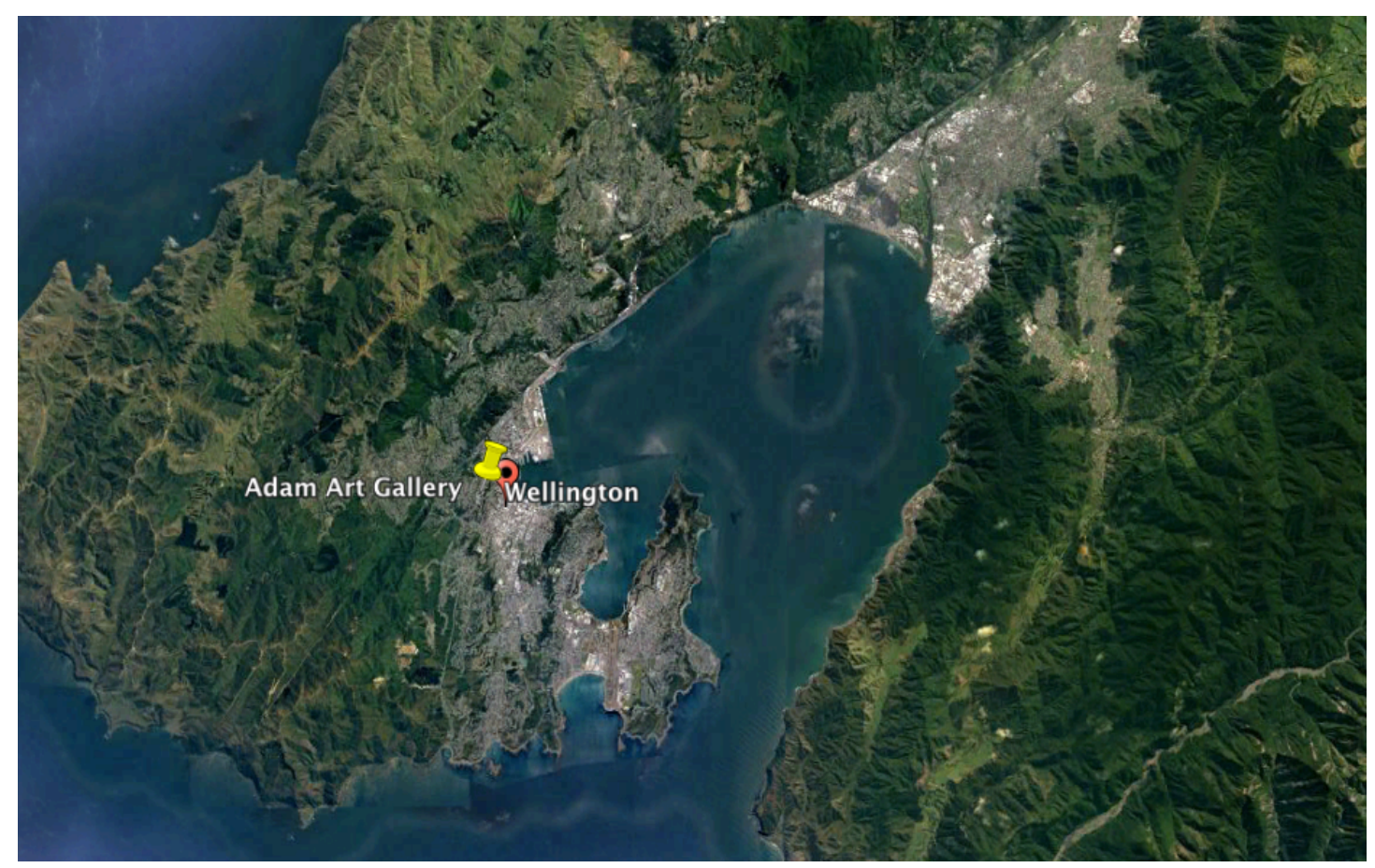

Figure 9: Google, Digital Globe 2016

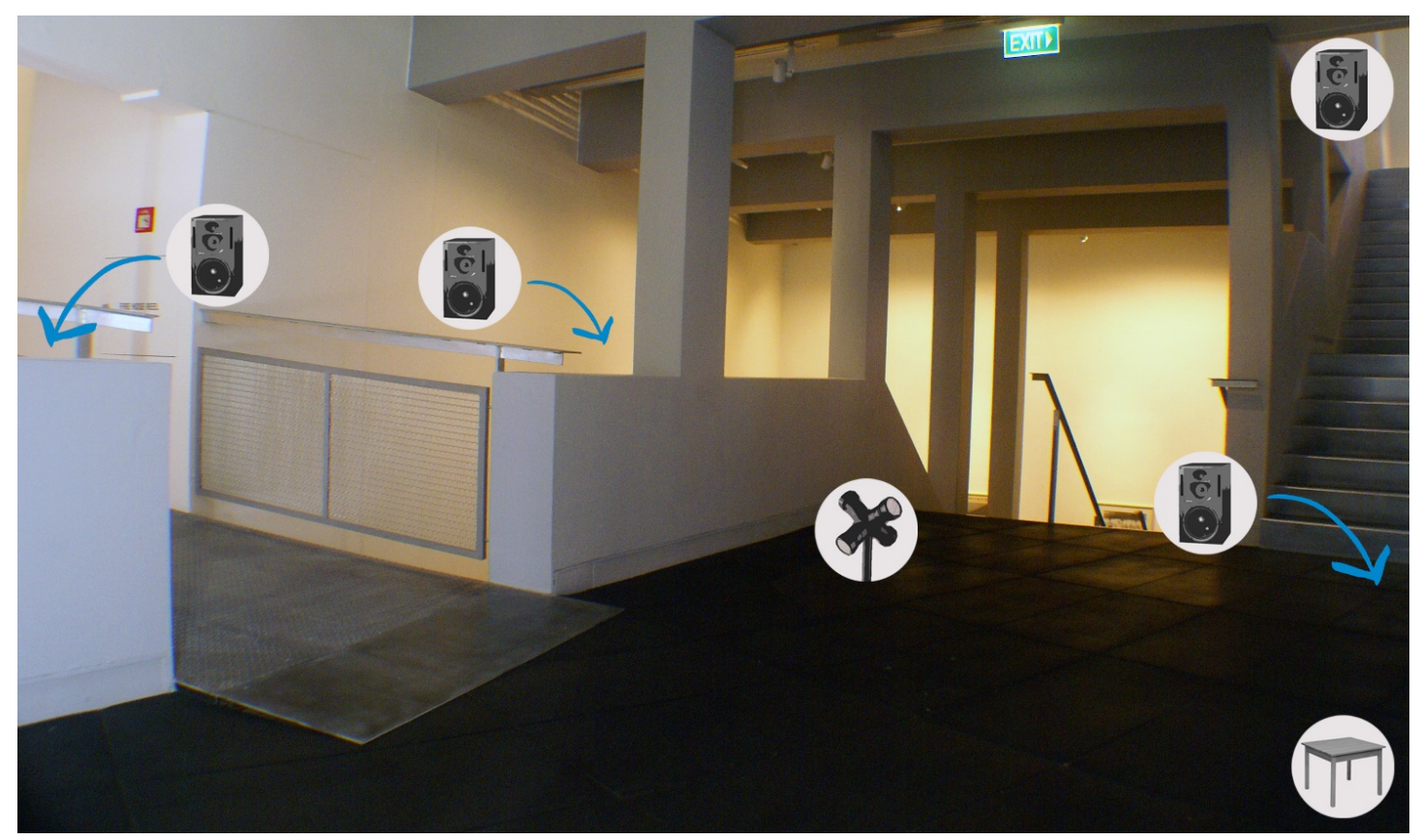

Figure 10: Adam Art Gallery Configuration - Photograph: Thomas Voyce; Icons and layout: Jina Yoon 
PARTIAL LIST OF EQUIPMENT ON TABLE (excluding cables, adapters etc):

- Macbook Pro laptop powered by Hyper Juice external battery pack

- Pro Tools software (diffusion and B-Format recording)

- Reaktor software platform running Synthi-In-A-Case VSTi

- Allen and Heath K2 Controller

- Metric Halo 8228 Audio Interface

SIGNAL PATH:

\section{1) Recording}

- ST450 microphone

- ST450 Preamp

- Metric Halo 8228 A/D converters

- Pro Tools Record Tracks (W, X, Y, Z)

\section{2) Diffusion}

- Software: Pro Tools with Reaktor Plug-In (Synthi-In-A-Case VSTI)

- MIDI control (K2 Controller)

- Discrete outputs (Metric Halo 8228 D/A channels 1-4)

- Wired connection to 4x Genelec 1037C Loudspeakers 


\section{TRACK 4}

Title:

BFMTPHNO-003 (2015)

Duration:

3'25"

\section{Format:}

B-format phonograph with improvisation on site

Location:

Point Howard Water Reservoir, Lower Hutt

This piece involves a 4-channel improvisation captured with a Soundfield ST450 microphone. The improvisation occurred at the Point Howard Water Reservoir, a site that overlooks the large industrial area of Seaview, Lower Hutt. The Soundfield microphone sat in the middle of a grass clearing next to the large concrete reservoir tank. The microphone extended 3 meters into the air, and faced down the steep hill toward the industrial area. Four Soundcast speakers encircled it in a quadraphonic array. I set up two microphones (2xAKG c451b), one facing down the hill (capturing the activity of the industrial zone) and one facing bush (capturing bird song and other natural features). The signals of these two microphones were recorded, and then pitch-shifted down an octave, with the related effect of slowing the sounds to half speed. The processed audio was then played back into the environment, which has the effect of extending the range of atmospheric noise down an octave, while relocating some of the very high pitch content into the more easily heard upper-mid range of human hearing, at half the original speed.

The piece begins with these processed recordings intermingling with the distal noise of the industrial zone. Over the course of the piece, the processed sounds decrease in amplitude, and are eventually silenced, leaving only the sound of the environment. Spatially, the piece is immersive, with little distinction between proximate and distant sounds. This particular recording was made on an early spring morning, the frost from the night before was melting as the sun rose over the steep hill behind. Quiet micro events, including defrosting water dripping from nearby trees, unprocessed bird sound and the occasional truck reversing 
indicate that the environment in audition is 'real', though my intention is that the mixture of environment and intervention remains ambiguous.

This piece has been inspired to some extent by the environmental 'land art' sculptures of Andy Goldsworthy (Goldsworthy, 2015). In many of his works, Goldsworthy manipulates materials found in an environment creating intricate and temporary sculptures. The environments he works in often deconstruct the resultant sculptures. His methodology has been documented in the film Rivers and Tides: Andy Goldsworthy Working with Time (2001). In this piece, the sun melts the frost on the tree leaves, creating a new sound as they hit the wet grass below. As this new sound appears, my intervention, itself a kind of sonic sculpture, disappears, leaving the environment as it was before I arrived.

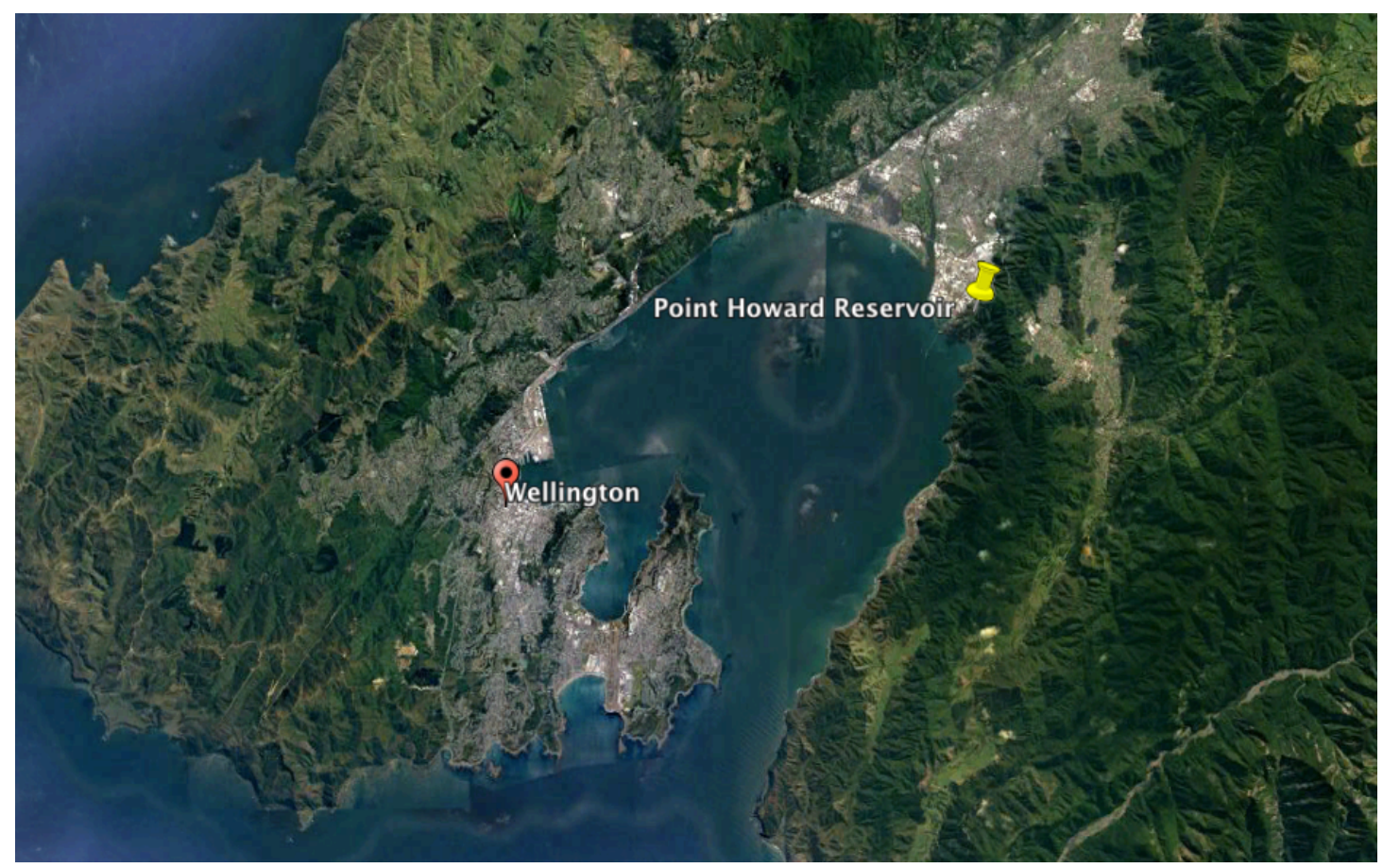

Figure 11: Google, Digital Globe 2016 


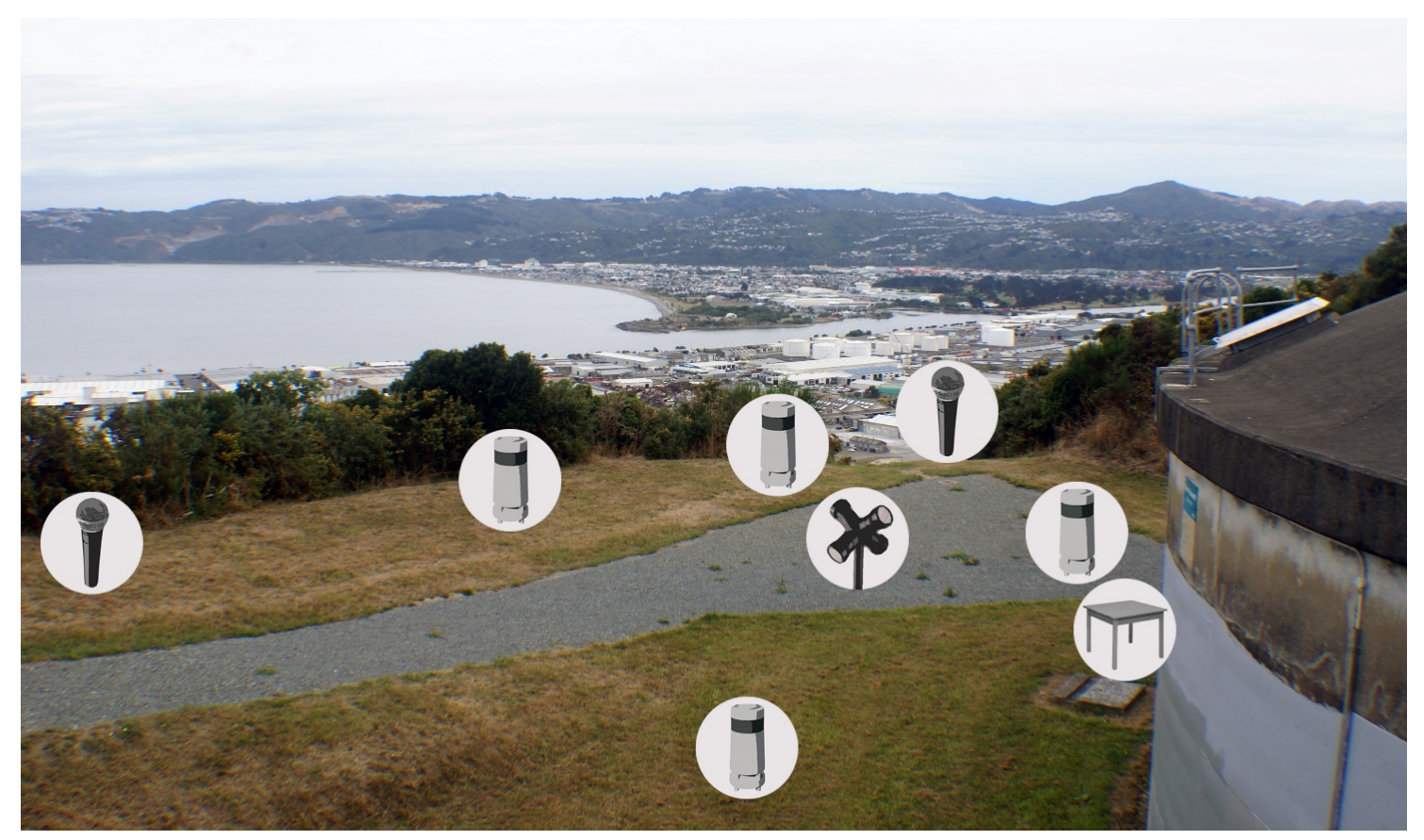

Figure 12: Point Howard Configuration - Photograph: Thomas Voyce; Icons and layout: Jina Yoon

PARTIAL LIST OF EQUIPMENT ON TABLE (excluding cables, adaptors, etc):

- Macbook Pro laptop powered by Hyper Juice external battery pack

- Pro Tools (audio diffusion and B Format recording)

- Allen and Heath K2 Controller

- Metric Halo 8228 Audio Interface

SIGNAL PATH:

\section{1) Recording}

- ST450 microphone

- ST450 Preamp

- Metric Halo 8228 A/D converters

- Pro Tools Record Tracks (W, X, Y, Z)

\section{2) Diffusion}

- Environmental capture: 2x AKG c451b small diaphragm condensors

- 2x Preamps (Metric Halo 8228) 
- Software: Pro Tools

- MIDI control of re-pitched recording volume (K2 Controller)

- Discrete outputs (Metric Halo 8228 D/A channels 1-4)

- Wireless connection to Soundcast Outcast loudspeakers 


\section{TRACK 5}

Title:

BFMTPHNO-004 (2015)

Duration:

4'36"

Format:

B-format phonograph with improvisation on site

Location:

Burdens Gate, Eastbourne, Lower Hutt

This piece comprises an improvisation through four speakers at the shoreline near Burdens Gate, Eastbourne. The performance is captured by the Soundfield ST450 microphone, which is raised 3 meters and directed towards the water. 2 hydrophones are used in this work, buried in the stones and shingle at the shoreline. These hydrophones are processed live through a combination of comb-filters and reverb, producing a drone-like effect, the volume of which is controlled with faders. The speakers are not in a quadraphonic array like previous works, instead they are positioned in a straight line, three meters between each, running parallel to the shoreline. The ST450 microphone is positioned between the speakers and the water.

The initial aim of this improvisation was to use the sound of the stones and shingle beneath the waves to generate new but related materials in the environment through the speakers. This is indeed the result in the first section of the work. However, there were some unexpected results. The first is that the speakers, perched along the ridge of a bank of stones and shingle (created by the high-tide), began to disturb the terrain as the amplitude of the speakers increased. The result is that the shingle began to cascade down the bank during louder sections of the improvisation, providing interesting micro detail and a new proximate space. These are the kind of unexpected results that occur when working with environmental sound in situ: not only can the environment itself produce unexpected sounds, but the audible interaction between the speakers and the physical environment can also. 
The second, more dramatic and equally unexpected result derives from the technology used in the improvisation. As the battery in my computer began to run out of power, the computer struggled to provide bus power to my audio interface. The result is a noticeable digital artifact, a kind of rhythmic digital glitch that I could not hear in situ, but was able to easily determine on playback, as it only affected the Soundfield ST450 recording. In my view, this adds a great deal to the work. Without my intention, the sonic signatures of the recording system make themselves known through the phonograph. In many respects, this piece represents an intervention not readily observed in other pieces in this portfolio. In most cases, my recordings of these improvisations are designed to promote the documentary feature of the phonograph, in much the same way classical recordings do. In this case, the process of abstraction is made apparent, unintentionally so. In this way, while I improvise with the environment, the phonograph also 'improvises', adding its own spatial, spectral and timbral gestures to the piece.

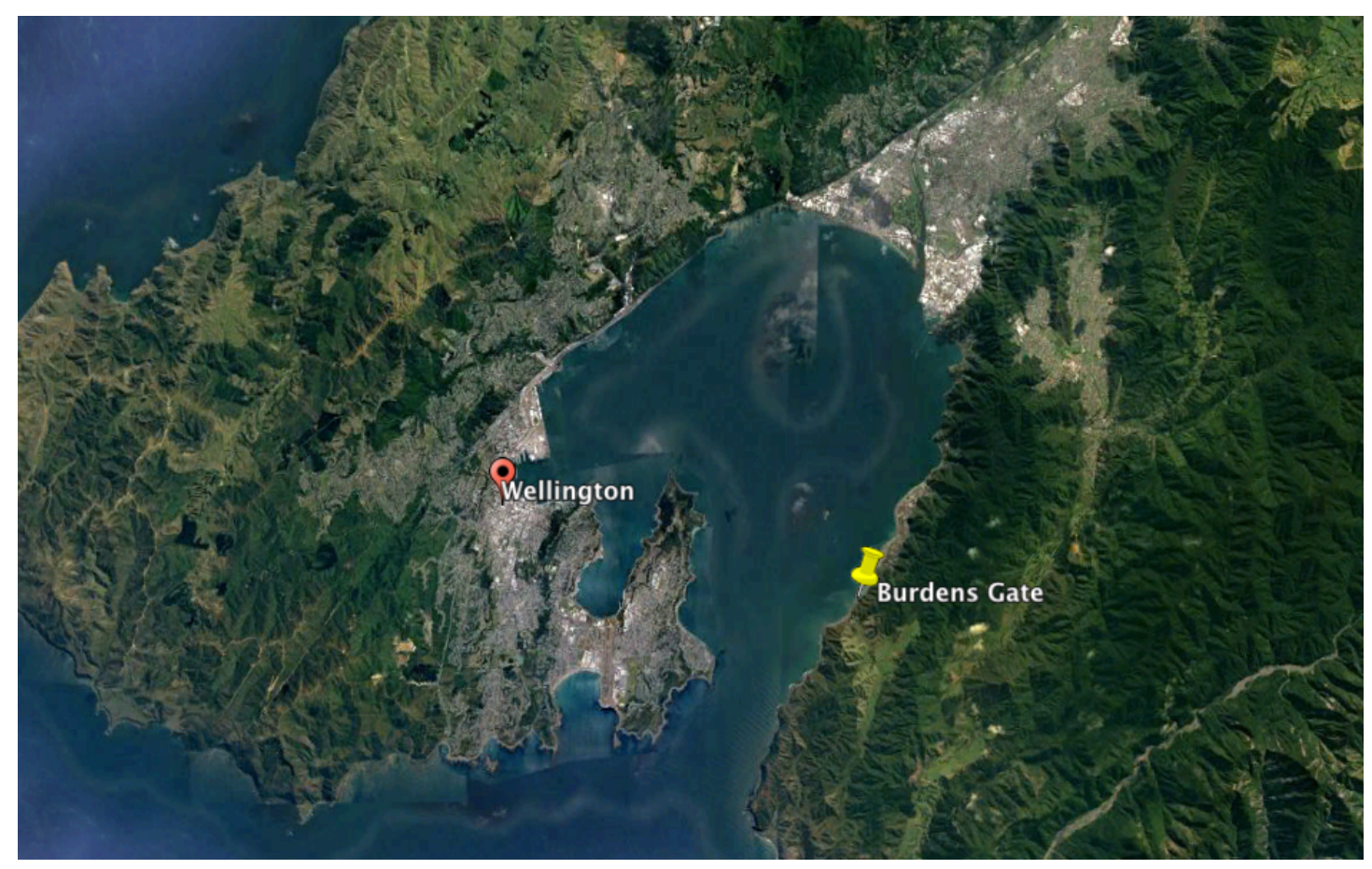

Figure 13: Google, Digital Globe 2016 


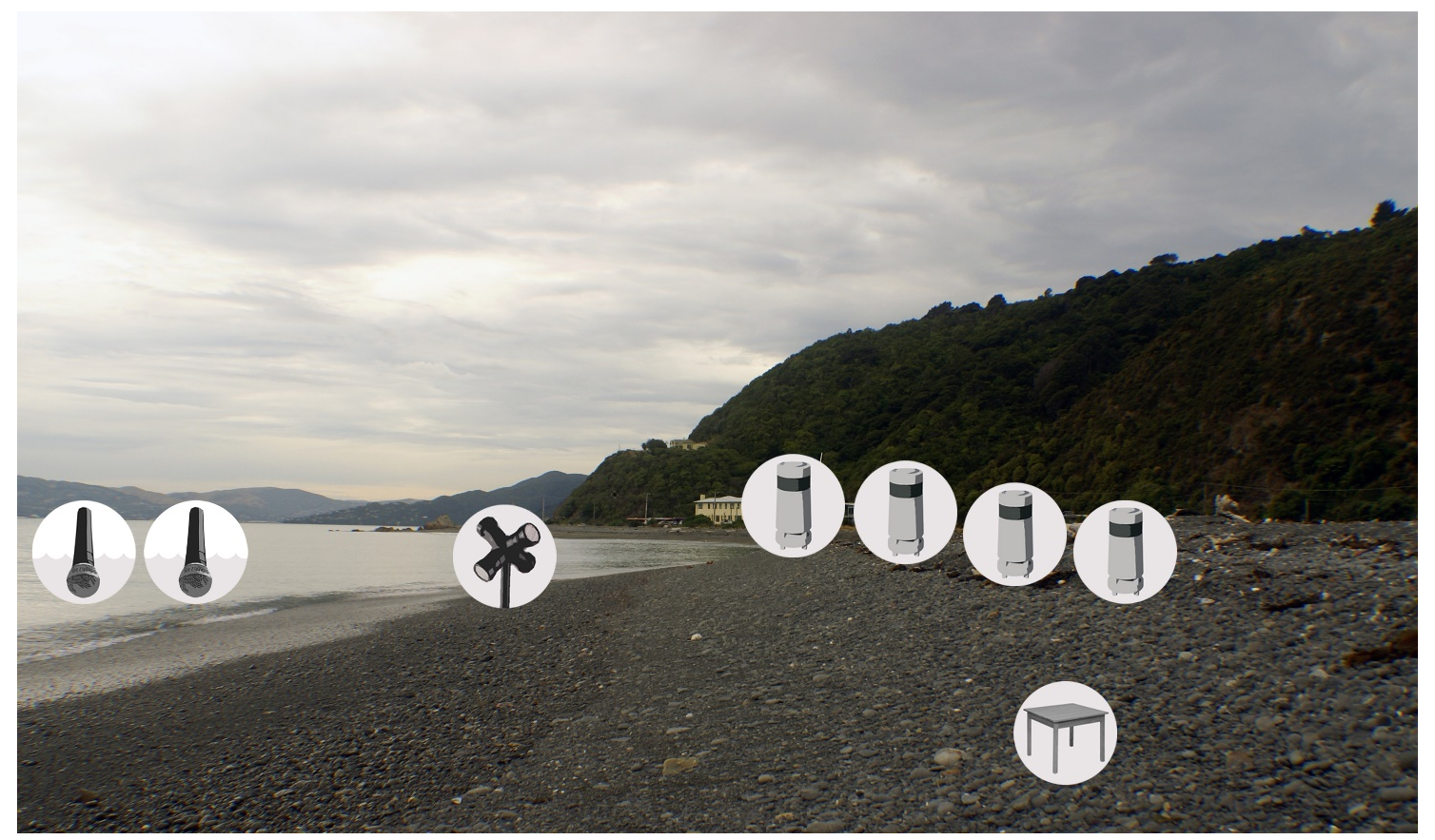

Figure 14: Burdens Gate Configuration - Photograph: Thomas Voyce; Icons and layout: Jina Yoon

PARTIAL LIST OF EQUIPMENT ON TABLE (excluding cables, adapters etc):

- Macbook Pro laptop

- Pro Tools (audio diffusion and B Format recording)

- Allen and Heath K2 Controller

- Metric Halo 8228 Audio Interface

1) Recording

- $\quad$ ST450 microphone

- ST450 Preamp

- Metric Halo 8228 A/D converters

- Pro Tools Record Tracks (W, X, Y, Z)

\section{2) Diffusion}

- Environmental capture: 2x Cold Gold Hydrophones

- A/D Conversion (Metric Halo 8228)

- Software: Pro Tools with Reaktor VSTs (Anima feeding Spacemaster 2)

- MIDI control (K2 Controller)

- Discrete outputs (Metric Halo 8228 D/A channels 1-4)

- Wireless connection to Soundcast Outcast loudspeakers 


\section{TRACK 6}

Title:

BFMTPHNO-005 (2015)

Duration:

5'58"

Format:

B-format phonograph with improvisation on site and post production

Location: Williams Park, Days Bay, Eastbourne.

This phonograph was recorded on a very windy night at Williams Park, Days Bay, Eastbourne. The Soundfield ST450 microphone was positioned in the middle of the park, with four speakers placed in a quadraphonic array around the microphone. I used the Synthi VSTi as the instrument for improvisation. On this particular evening, and while I was setting up my equipment, the wind changed from a dead-still south easterly, to a very strong north westerly, the prevailing wind of the Wellington region. My intention had been to record quiet sounds in a quiet, expansive space, but the change in environmental conditions meant I too needed to change my plans.

The 'noise' component of the environment is easily heard; the sound of the wind in the trees and the waves crashing along the nearby beach create a swirling white noise-like texture, within which the improvisation occurs. In order to cut through this noise, I needed to make strong gestures with the Synthi (loud, full range gestures). When I listened to the results later in the studio, I decided that the interesting aspect of the recording was not the spatial aspect of the environment, nor the spectromorphological content of my improvisation (as had been the intention in situ). I was, instead, attracted to the quality of the noise. To begin with, it was unclear to me if the noise component of the work was technological or environmental, especially at the beginning of the work. In fact, the majority of the noise is environmental, though the noise floor of the recording system is also audible (only just). This led me to consider that, despite 
my initial intentions, the interest in this recording resides with the ambiguity of the noise component. As such, I looked for ways to extend this idea.

In post-production, I decided to add some saturation to the signal to further emphasise the noise elements of the work. When the louder gestures from the Synthi come in, they distort with increased harmonic saturation. At the beginning of the work, it is unclear if the noise is environmental or technical, and when the Synthi distorts, a listener might hear the noise as produced by technology. However, as the piece concludes, the iterative nature of the waves on the beach becomes more pronounced, exposing the environmental contribution to the noise. The noise element in this piece is thus both environmental and technological. In this piece, like the last, the recording of the improvisation has been further enhanced, this time intentionally, which has highlighted the role of abstraction in the work.

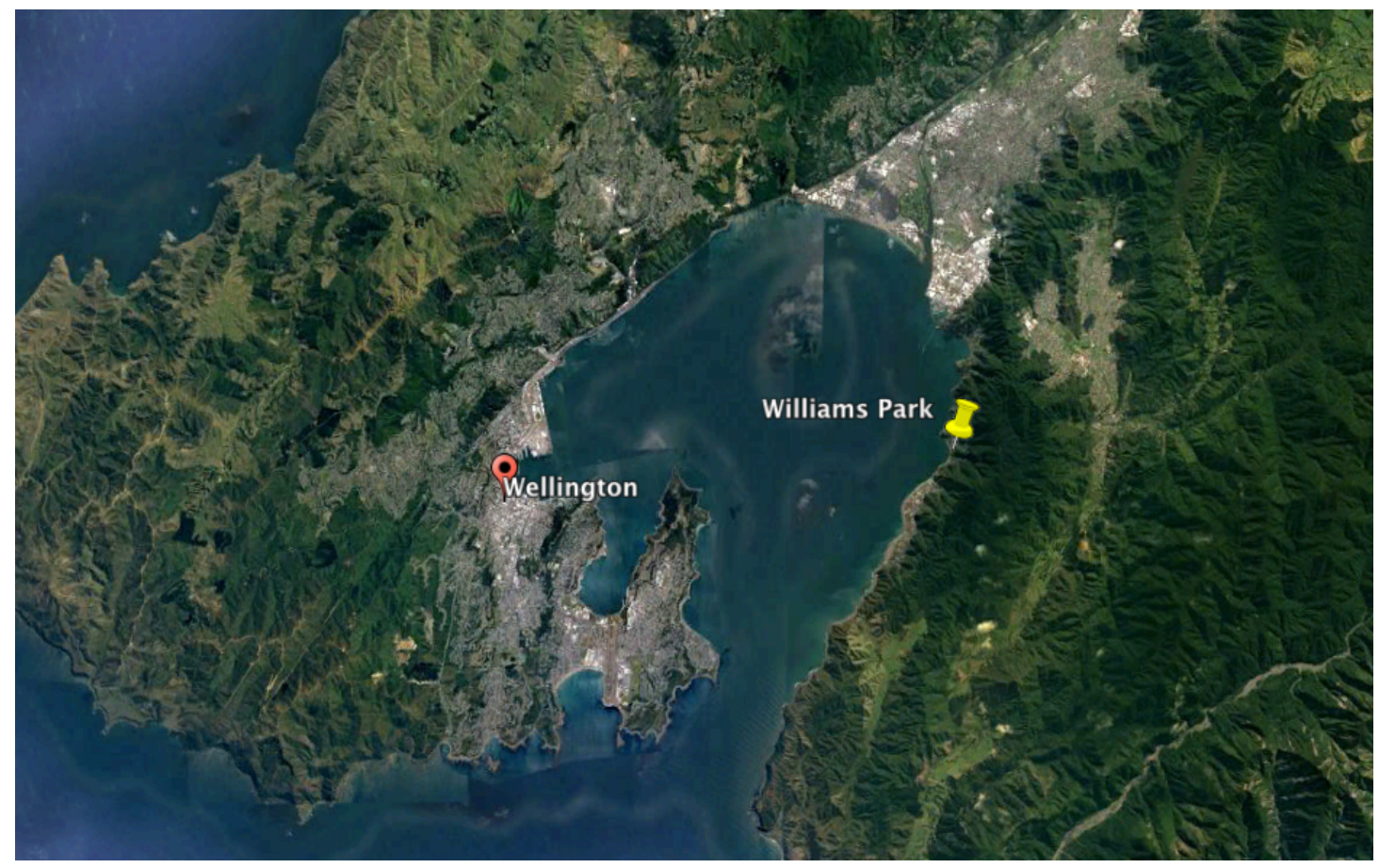

Figure 15: Google, Digital Globe 2016 


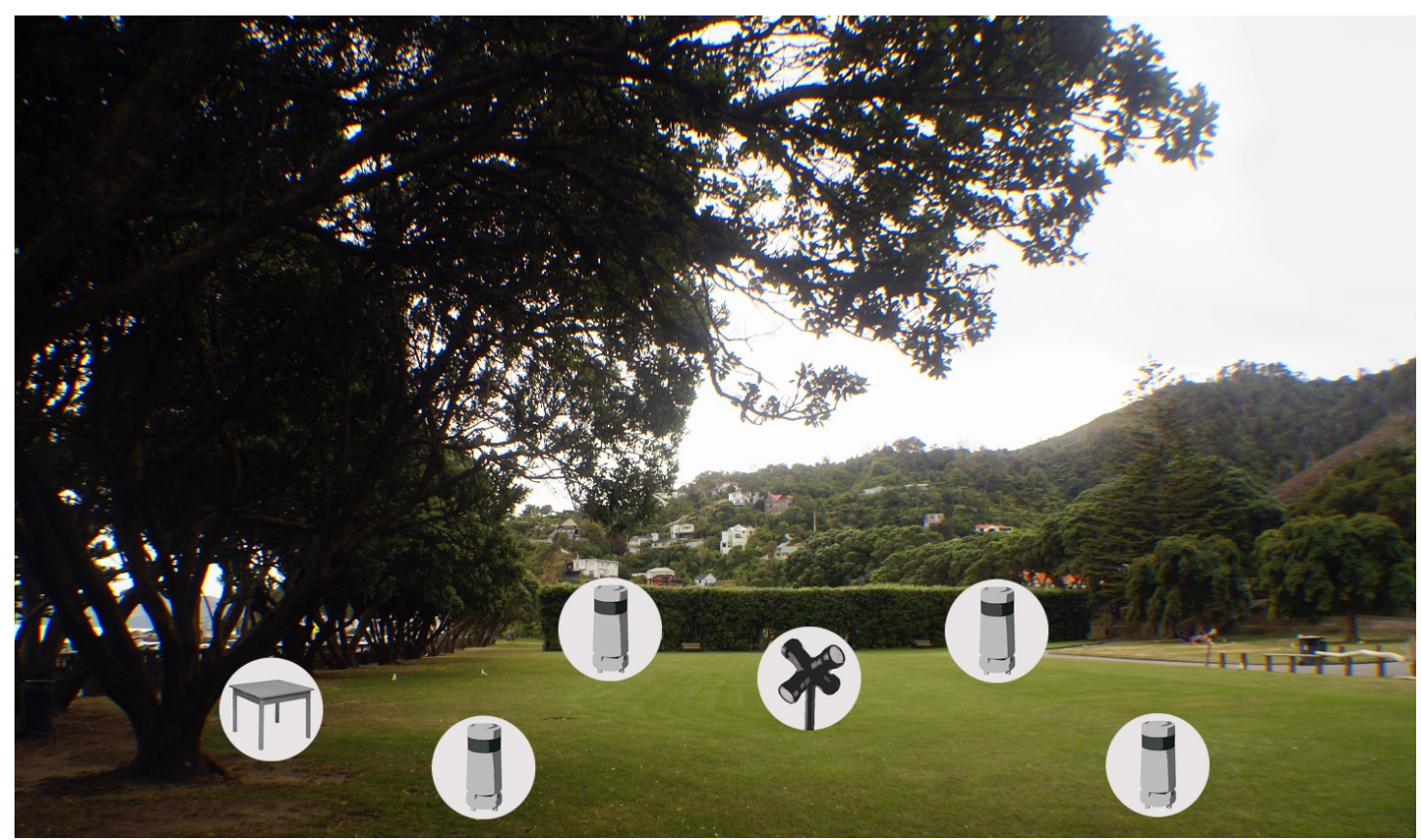

Figure 16: Williams Park Configuration - Photography: Thomas Voyce; Icons and Layout: Jina Yoon

\section{PARTIAL LIST OF EQUIPMENT ON TABLE:}

- Macbook Pro laptop

- Pro Tools (audio diffusion and B Format recording)

- Allen and Heath K2 Controller

- Metric Halo 8228 Audio Interface

\section{1) Recording}

- ST450 microphone

- ST450 Preamp

- Metric Halo 8228 A/D converters

- Pro Tools Record Tracks (W, X, Y, Z)

\section{2) Diffusion}

- Software: Pro Tools with Reaktor VSTi (Synthi-In-A-Case)

- MIDI control (K2 Controller)

- Discrete outputs (Metric Halo 8228 D/A channels 1-4)

- Wireless connection to Soundcast Outcast loudspeakers 


\section{TRACK 7}

Title:

Duration:

Format:

Location:
2CHPHNO-003 (2015)

$11^{\prime} 7^{\prime \prime}$

Stereo phonograph with improvisation on site

York Bay, Eastbourne.

\section{Description:}

Like the previous five pieces, this piece is composed of a single take recording of an improvisation in situ, though this work is diffused in stereo, captured by a middle and side microphone configuration, and unlike the previous piece, there is no post production. The recording was made in the summer of 2014/2015, one kilometer from the York Bay entrance into the East Harbour Regional Park, in Lower Hutt. I routed a microphone, placed $300 \mathrm{~mm}$ above stream, through a variable comb-filter (controlled live a MIDI controller), into a reverb with an infinite decay. I also set up a white noise generator on a separate channel. My third channel contained the Synthi VSTi. I controlled the parameters of this software synthesiser with extensive MIDI mapping on the Allen and Heath K2, as I have done with other pieces that involve this instrument.

The recording of this environment was made with Sennheiser RF condenser microphones into a Sound Devices 722 recorder, a system that display an incredibly low noise floor and excellent frequency response. The speakers form an equilateral triangle with the microphones (at a distance of 3 meters) to preserve the stereophony of the effects and synthesiser. In this piece, it was my intention to capture an excellent (high detail) stereo image given the performance of the equipment used, and the relatively restricted spatial profile of stereo when compared with ambisonic capture. In my view, this piece is successful from this perspective.

As the environment was full of the sound of cicadas, creating a pulsating white noise effect, my first decision was to reveal this texture by masking it with white 
noise, played at high levels back into the environment through the Soundcast speakers. The piece begins abruptly in white noise. I decreased the level of white noise over time, allowing the sound of the cicadas to become present. This might be understood as an unveiling of the environment through technology. I then introduced expansive textures into the environment, born of the filter/reverb combination described earlier. A fly inspects the microphone capsules on a number of occasions, adding extreme foreground and 'microphone space' (Smalley, 2007) to what is largely a distal environment. The stream, occupying an intermediate space between the fly and the cicadas presents a number of interesting sounds, which I emulate and extend using the Synthi VSTi. At the end of my improvisation, I introduce the white noise back into the environment, gradually increasing the volume until it masks the environment, thus framing my improvisation and the resultant phonograph at the beginning and end.

The use of white noise in situ defines and extends the inevitable non-site framing that occurs when the temporal frame of a recording - the beginning and end - is selected in post-production. In many of my improvisations, I begin performing when something attracts my ear, and later, I will include this aspect when I frame the work, often by fading in the recording sometime before the improvisation begins. In this case, I deliberately define the frame to be used by projecting white noise into the environment at the beginning and end of the work. This has an interesting effect, in that it is difficult to tell, at least at the beginning of the piece, if the white noise is environmentally embedded or if it is layered over the recording in the studio. As such, like the previous piece, a certain ambiguity around technological and environmental noise is explored.

This piece is also the one most conceptually related to the work of Douglas Lilburn. In Soundscape with Lake and River (1979), a work in which the Synthi plays a central role, Lilburn uses the water sounds of Lake Taupo, and sets them in a kind of counterpoint with the Synthi. My intention in this work is to shift the site of composition from the studio to the environment, in a maneuver that I consider to be an extension of Lilburn's methodology. 


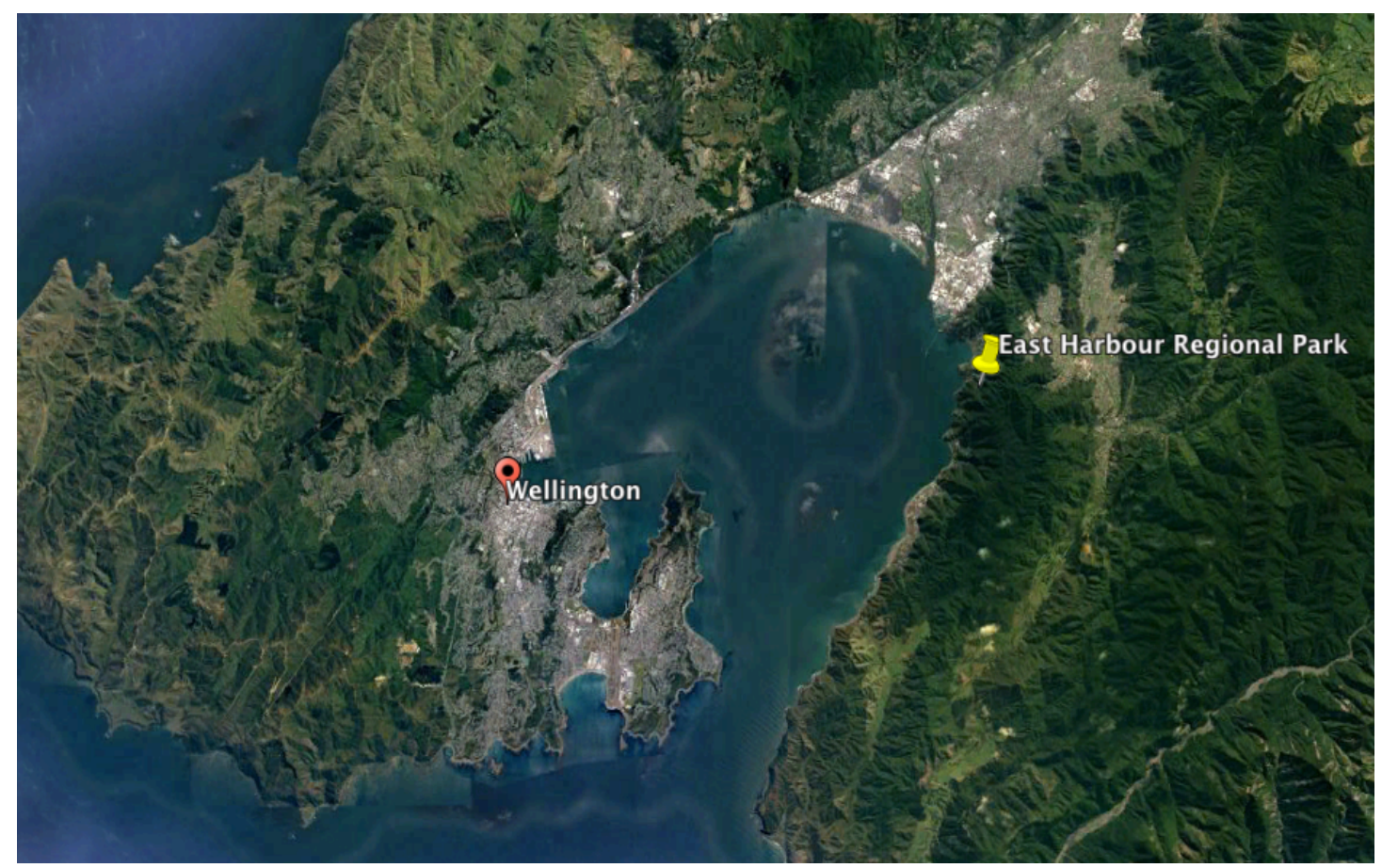

Figure 17: Google, Digital Globe 2016

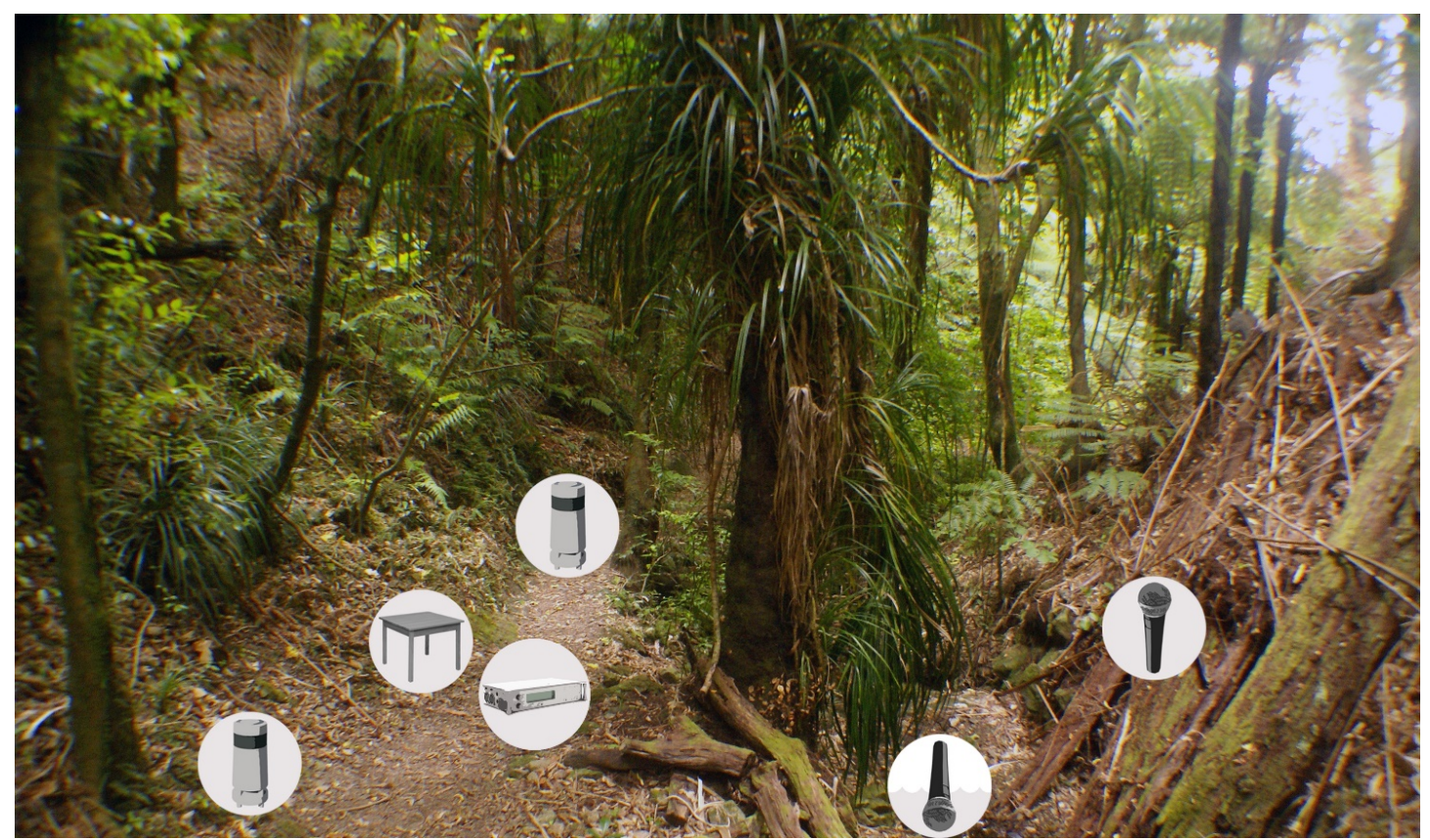

Figure 18: East Harbour Reserve Configuration - Photograph: Thomas Voyce; Icons and Layout: Jina Yoon

\section{PARTIAL LIST OF EQUIPMENT ON TABLE:}

- Macbook Pro laptop powered by a Hyper Juice external battery pack

- Pro Tools (white noise generation, audio diffusion)

- Reaktor software running Synthi-In-A-Case VSTi

- Allen and Heath K2 Controller 
- Metric Halo 8228 Audio Interface

- Sound Devices 722 recorder

\section{1) Recording}

- Sennheiser MKH 20 P48 + Sennheiser MKH 30 P48 in M/S Configuration

- Sound Devices 722 digital recorder

\section{2) Diffusion}

- Software: Pro Tools (White Noise Generator) with Reaktor (Synthi-In-ACase, Amine, Spacemaster 2)

- MIDI control (K2 Controller)

- Discrete outputs (Metric Halo 8228 D/A channels 1-2)

- Wireless connection to Soundcast Outcast loudspeakers 


\section{TRACK 8}

Title:

Duration:

Format:

Location:
2CHPHNO-002 (2015)

$11^{\prime} 22^{\prime \prime}$

Stereo phonograph with additional recording

Terrace Tunnel, Te Aro, and Eastbourne, Lower Hutt.

This piece deals directly with the meta-document of environmental sound composition, in which the notion of the event and the location of the composer are explored. The recording was made in Wellington, during the summer of 2014/2015 in the early hours of the morning above the Terrace Tunnel, a busy location along State Highway 1 for vehicles travelling to and from Wellington city. For this recording, like the last, I used a Sennheiser MKH 20-P48 and a Sennheiser MKH 30-P48 in Middle and Side configuration, recording onto a Sound Devices 722 field recorder for a precise stereo field, extremely low noise floor, and a full frequency range. There is no improvisation in situ, the recording is devoid of such intervention. The recording made is also unedited, and presented as such in this piece.

The recording has then been routed through a stereo channel of an Allen and Heath Xone 92 mixer. Hardware effects have been applied to the audio, using the auxiliary busses and the headphone output as a subgroup. The effect loops used include a Mu-Tron Bi-Phase feeding a Korg GR-1 spring reverb, a Roland RE-201 tape echo and a Eventide H3000 digital effector set to a custom algorithm that uses a mixture of comb-filtering and reverberation. Using headphones for monitoring, I then played the Terrace Tunnel recording through the mixer, while improvising with the mixer and effects, recording the outputs of the mixer into a DAW. My improvisation uses techniques commonly found in dub mixing, particularly in the use of spring reverb, tape delay and modulation effects. Only the effects channels were recorded. Using the same Sennheiser microphones, I also recorded the room sound in which this improvisation took place - a spare bedroom in my house that doubled as a temporary home studio. These 
microphones picked up the sound of my improvisation without the fruits of it; that is, the sounds of my shuffling from one piece of technology to another, flicking switches and turning dials. The mechanical sound of the equipment is also present in this recording, the tape machine is particularly noisy as the tape spools around the unit. After the improvisation is complete, I open the window and switch off equipment.

The piece is thus composed of three core materials: the original recording, the separately recorded effects, and the room sound of my home studio. The piece begins with the original recording. Increasingly, the effects become apparent (as per the original improvisation). By $08^{\prime} 00^{\prime \prime}$, the original recording has disappeared from the mix, and we hear only the effects channel. By 09'00", the room sound is introduced - a relationship between the audible manipulations of equipment and the auditory effect of such manipulations is established, before the room recording takes over altogether, concluding with sound of the environment outside my home studio coming in through the open window.

The piece seeks to make explicit what is often muted in environmental sound composition: the presence of the composer and further, the location of their composing. In this piece, a recording is at first presented much like many works of the genre of phonography. At this stage of the piece, the composer is perhaps understood as present within the environment. Manipulation is introduced, subtly at first, and then with more vigor. The composer is then understood as existing temporally between the moment of capture and the moment of audition. This vague temporal location is then given a concrete physical location as the room sound enters the piece. The baton passes from the original site of recording to the second site of composition, exposing the multiplicity of environments in much environmental sound composition. It is my hope that such a movement may result in a listener considering their own environment at the conclusion of the piece, thus completing the set of environments that are implicit in the production and audition of many environmental sound compositions. 


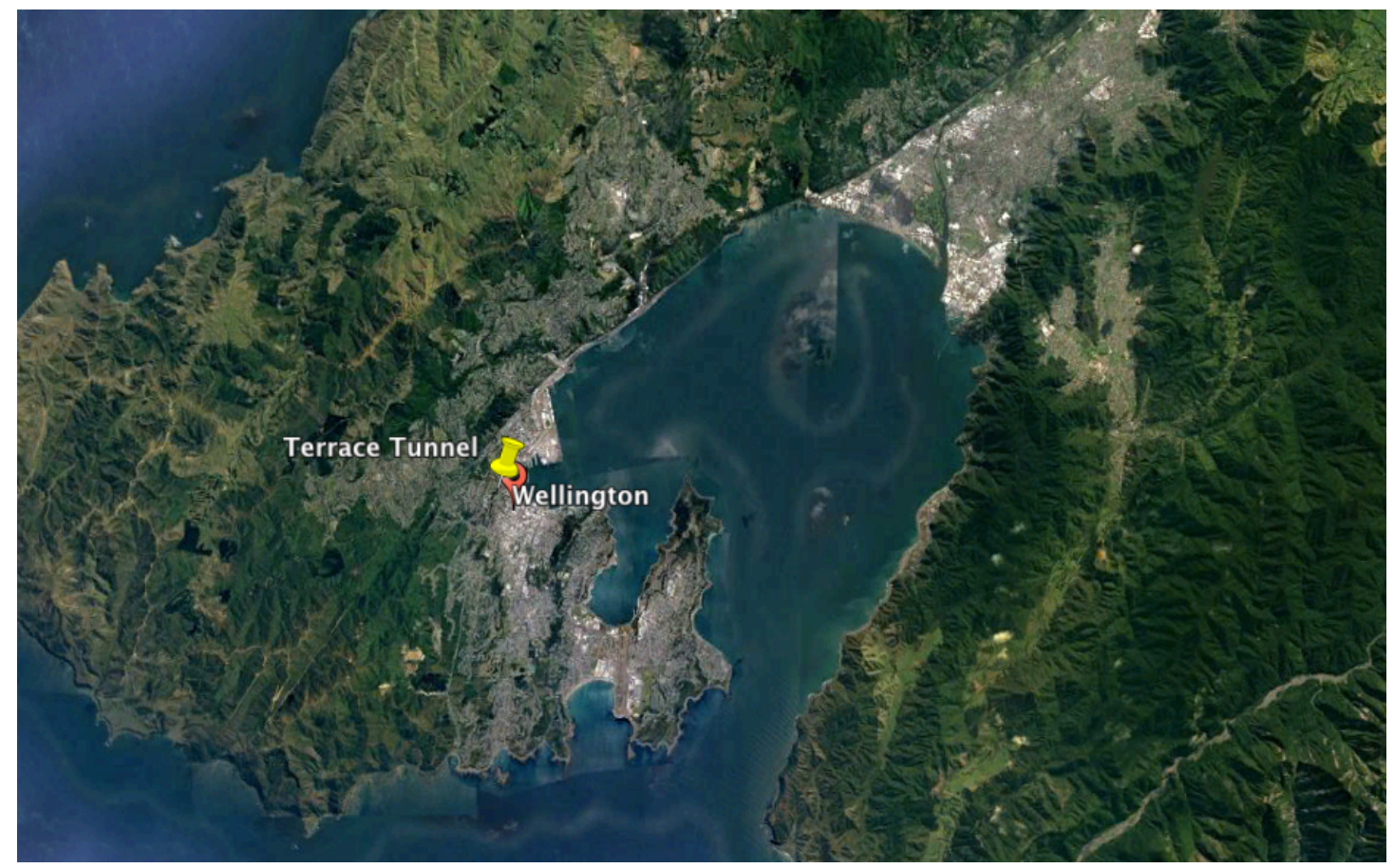

Figure 19: Google, Digital Globe 2016

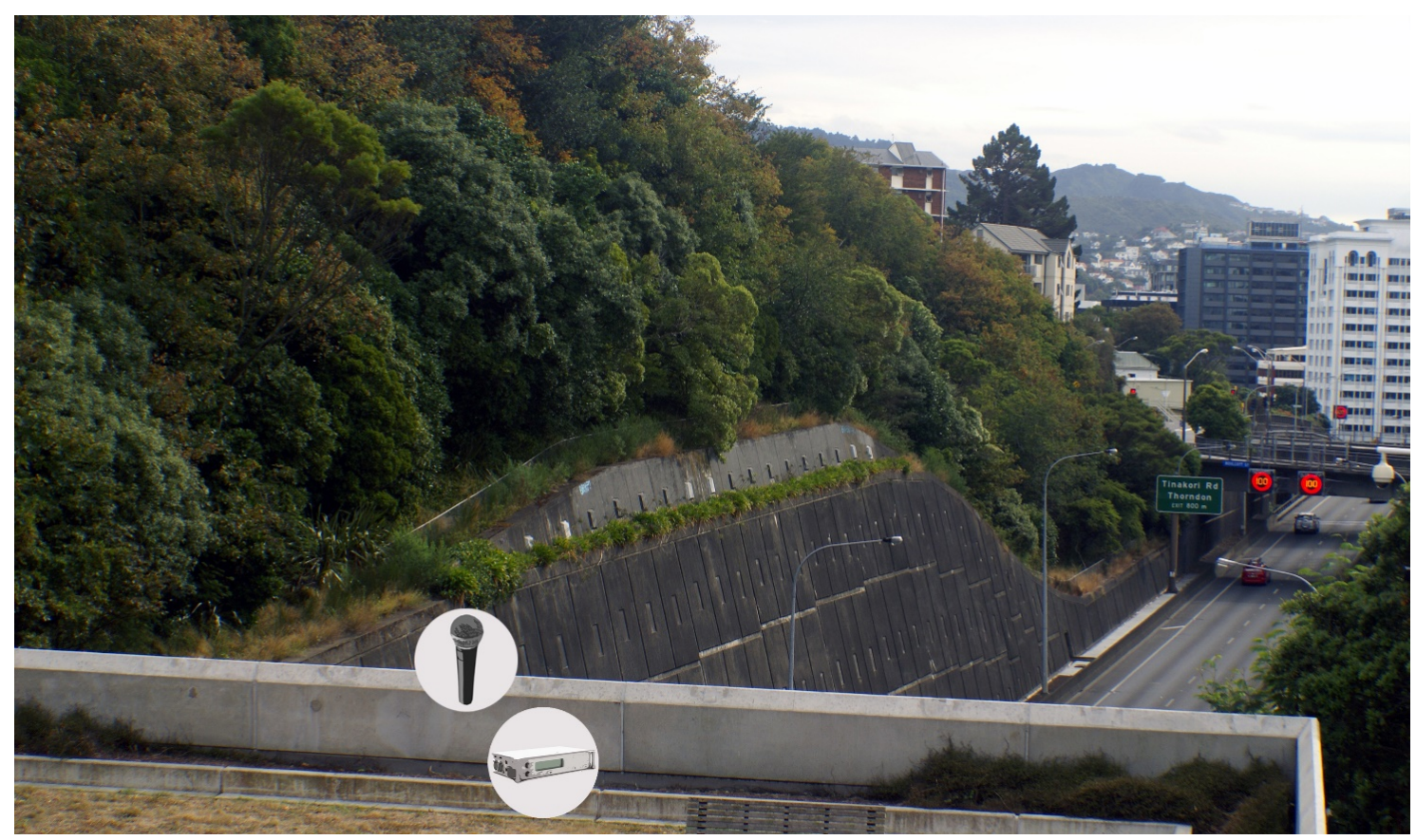

Figure 20: Terrace Tunnel Configuration - Photograph: Thomas Voyce; Icons and Layout: Jina Yoon

NB: My home studio was also used in the creation of this piece. Photograph and location not provided. 
PARTIAL LIST OF EQUIPMENT USED IN HOME STUDIO:

- Macbook Pro laptop

- Pro Tools (recording and diffusion via headphones)

- Allen and Heath Xone 92 mixer

- Metric Halo 8228 Audio Interface

- Roland RE 201 Space Echo (tape delay)

- Mu Tron Bi Phase (phasor)

- $\quad$ Korg GR-1 (spring reverb)

- Eventide H3000 (digital multi-effects unit)

- $\quad$ Sennhesier MKH 20-P48

- $\quad$ Sennheiser MKH 30-P48

\section{1) Recording:}

- Sennheiser MKH 20 P48 + Sennheiser MKH 30 P48 in M/S Configuration

- Sound Devices 722 digital recorder (Terrace Tunnel)

- Pro Tools (home studio)

\section{2) Diffusion}

- Terrace Tunnel recording sent from Metric Halo 8228 (Channels 1-2)

- Received by Allen and Heath Xone 92 (Stereo channel 1) output muted

- Aux 1 (pre-fade mode) sent to Roland RE201 Space echo, returned to Xone 92

- Aux 2 (pre-fade mode) sent to Mu-tron Biphase, then Korg GR-1, returned to Xone 92

- Headphones sent to Eventide H3000, returned to Xone 92.

- Output of Xone 92 (effects only) recorded to Pro Tools, combined with Sennheiser M/S recording (home studio) and original recording (Terrace Tunnel) and arranged in Pro Tools 


\section{TRACK 9}

Title:

Duration:

Format:

Location:
2CHPHNO-001 (2015)

7'36"

Stereo phonograph with post production

Yeoju, Gyeonggi Province, South Korea

This piece is composed of a single take stereo phonograph, recorded in Yeoju, Gyeonggi-do, South Korea in April 2015. The phonograph was recorded live onto cassette tape using a Sony WM-D6C Professional Walkman (with Dolby B noise reduction) and a Sony ECM-MS957 electret microphone. The recording was then re-recorded (bounced) to cassette five times (using a digital recorder as an intermediary device), each time with a small increment in gain in order to saturate the tape and introduce noise: the first recording is 'clean', while the fifth recording is heavily distorted. All five recordings were then mixed together, in unison with varying levels; the beginning presents all five recordings at full level, and by the end of the piece, the original recording plays by itself. The other four recordings are faded out at various times throughout the piece. The temporal integrity of the original recording is intact (no editing), and no additional processing has been employed, such as equalisation, dynamic or spatial processing. As such, this piece, while heavily processed through layering, is in fact a single take phonograph.

This piece constitutes an exploration of my Sony WM-D6C's sonic signatures. Every tape machine is different given the mechanical nature of such devices, and mine, a 20-year-old unit in good condition, exhibits a number of characteristics. The first is a fairly stable quartz lock capstan servo system, which provides consistent tape speed. However, with a level of predictability, when the recordings are overlaid a small amount of wow and flutter (with occasional dropouts) is detectable, along with a very slow phasing effect, born of the slight differences in tape speed between recordings. This is most apparent at the beginning of the piece. The second feature of my Walkman is that it exhibits a 
very effective Dolby B noise reduction system: though I have re-recorded this original recording many times to tape, the noise floor (tape hiss) remains fairly inconspicuous, especially when compared with the tape saturation. The tape saturation is of particular interest, as it performs in a slightly unexpected way. I was anticipating a particularly resonant and distorted mid-range by the last bounce, but the tape has compressed this mid-range, allowing for a fairly even frequency response across all recordings. The end result is that the recordings, though distorted, have a pleasing mid-range, as well as an extend frequency response.

The original recording was made shortly before midnight on a dirt road overlooking a rice field in rural Yeoju. The location was chosen for a number of reasons. The first is that the wildlife presents interesting materials in terms of space, timbre and rhythmic interplay. It is also situated some distance from a busy road, along which many military vehicles travel to and from a military base located a few kilometers from the site. At this time of night, vehicles pass very rarely, and when they do they present a very distinct acoustic event, uncluttered by the sound of multiple vehicles. Lone vehicles at night usually produce a sound with the hallmarks of phasing and the Doppler effect as they pass. Given my predetermined process of overlaying the recordings together, I anticipated phasing to be a feature of the sonic signature of the technological procedure and hoped for some commonality between the materials of the environment and the effects of my methodology. As is often the case, this primary poietic intention though successful in some respects, presents just one of a multitude of relationships between the event and the technology employed. In fact, this particular poietic motivation now has very little to do with my appreciation of this work.

The final reason I chose this site was because of the unique spatial characteristics it presented: across the field some 70 meters away a large concrete wall, more than three meters high, had just been erected, the other side of which constitutes the grounds of a newly established 'religious community' according to a local resident. This wall provides a reflective surface that serves to define the spatial signature of the location. The sound of dogs at $01^{\prime} 10^{\prime \prime}$ and the 
siren, re-emerging at 2'30" engage this spatial attribute; they are, as Smalley (2007) might agree, bearers of space, and it is this aspect, not their sourcebonded signification, that presents me with the most interest in this sonic environment.

In particular I like the unveiling of space in this work. Though the piece starts in a cacophonous roar of distortion, the last 90 seconds are very pleasing to listen to. In fact the materials at the end are very similar to the materials at the start; it is the process of overlaying the recordings with increased distortion that creates this effect. As such, this piece explores abstraction and documentary in equal measure.

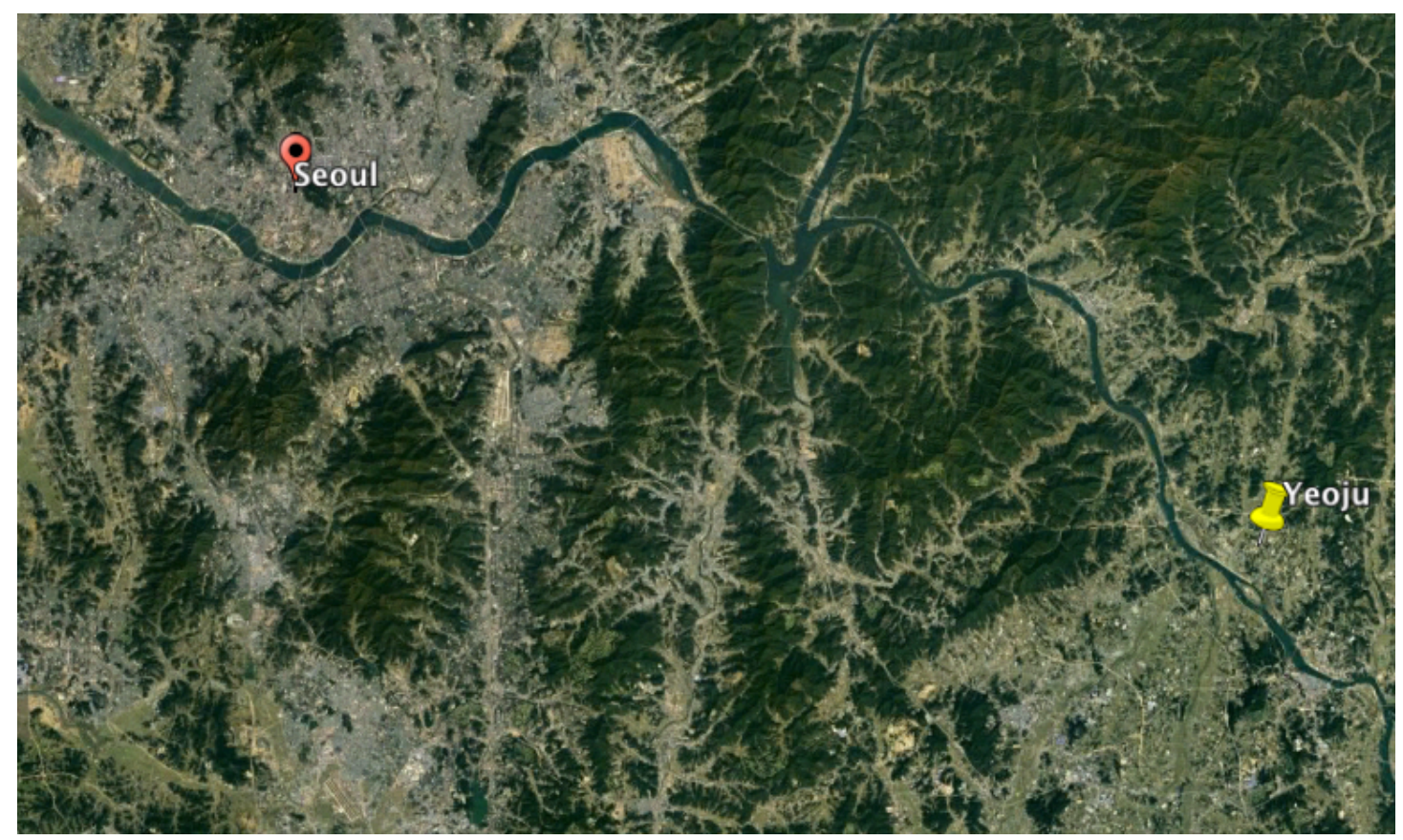

Figure 21: Google, Digital Globe 2016 


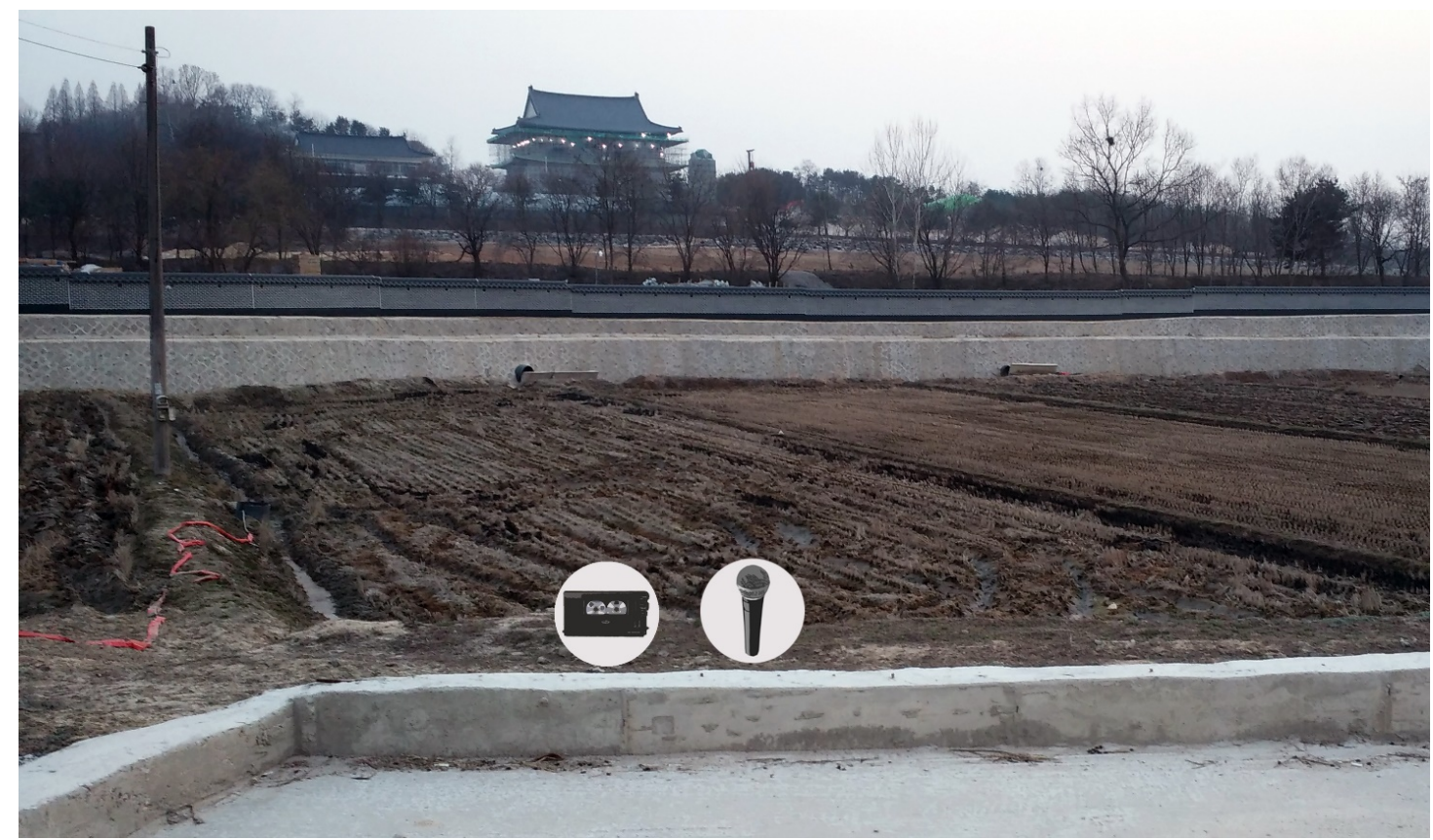

Figure 22: Yeoju Configuration - Photograph: Hong Mi Lee (used with permission); Icons and Layout: Jina Yoon

\section{PARTIAL LIST OF EQUIPMENT USED IN PRODUCTION:}

- Sony ECM-MS957 microphone

- Sony WM-D6C cassette recorder

- Sound Devices 722 digital recorder

- Macbook Pro

- Pro Tools 


\section{TRACK 10}

Title:

Duration:

Format:

Location:
2CHPHNO-004 (2015)

7'00"

Stereo phonograph

Seocho-gu, Seoul, South Korea

This piece comprises a triptych of field recordings, all made one Autumn evening in 2012 in Seocho-gu, Seoul, South Korea. Recorded in the hills that rise behind the Seoul Arts Center, the piece captures three distinct perspectives. The first perspective focuses on the chirping of a small insect, while a vehicle (one with audible mechanical problems) negotiates its way out of the car park below. The second section is focused to the city, the sounds of sirens engaging its aural architecture. An intoxicated man walks the footpath below, singing as he stumbles along. The final section returns to the chirping of the insect, though from a greater distance. The microphones are now closer to the source of music, played through loudspeakers, that permeates each section.

From a poietic perspective, this piece is concerned with framing: temporal framing, spatial framing and cognitive framing. To begin, the temporal frame I have imposed on these recordings, with the use of uniform fades, represents a deliberate attempt to endorse the documentary feature of the phonograph. In allowing the environmental recordings to emerge and recede in the piece, I attempt to promote an understanding of the environment as independent from the recording: it exists before and after the recording takes place. In other words, I use a technique of abstraction to promote the work's documentary feature. However, in presenting the piece as a triptych (a formal framing device often used in art and photography), I also promote the abstractive feature of the phonograph, and my role as an author of it. Presenting the work in three distinct sections tacitly suggests that relationships might exist between them. As a matter of a listener's noetic response to the framing device employed, 
relationships between sections might be divined, though I do not suggest what these relationships might be.

The spatial frames I employ include what Smalley terms perspectival space and vantage point shifts (2007). Though other spatial frames are present, including the previously detailed microphone space of 2CHPHNO-003 (2015), along with utterance space, panoramic space and distal space, I use perspectival space and vantage point shifts between sections as intentional framing devices, which in turn reveal these other spatial features. Each section presents a unique perspectival space. The first section has a very proximate space, which serves to mask the distal space of the city to a degree. The insect sound, located in the centre of the stereo field demands attentional focus at the beginning of the work, though my experience of this changes over time: I am able to cognitively tune out this sound as it becomes repetitive, instead focusing on the distal sounds that occupy the panoramic space of the image. The sound of the vehicle aids in this cognitive transition. With a change of section comes a shift in vantage point. The perspectival space of the second section has much less proximate features, allowing the distal sounds of the city, the sirens in particular, to highlight the aural architecture of the city. This panoramic perspective is interrupted by the intoxicated singer, who presents an utterance space. A second utterance space emerges, as a song begins to play on the large outdoor PA system some distance away. The final section presents a new vantage point. This time, the microphones are closer to the PA system, which is silent to begin with. The perspective is ambiguous, as there is less activity in this section than the preceding ones. The city hum and insect sounds do not present a clear foreground and background. An orchestral piece begins on the PA system (an unknown rendition of Ennio Morricone's piece “Gabriel's Oboe" from the film The Mission [1988]) and though it is clearer and louder than before, it still presents a distal space. The spatial atmosphere of this section is quite homogenous, and is only punctuated by the roar of a motorcycle engine towards the end of the work.

Though I do not presuppose how a listener will cognitively frame this work, I will make some observations from my own experience of it. As mentioned, the insect 
sound in the first section quickly becomes 'steady state' (Truax, 2001), and thus I am able to attend to other aspects of the environment. In the second section, I am able to focus more broadly along the temporal span (Ihde, 2007), though this is interrupted by the intoxicated singer. My attention is now focused to the futural edge of the piece as I listen to his voice in order to determine the content of his words and perhaps the state of his health. In the final section, I also listen with a broad focus along the temporal span, with an equally broad attentional focus. While this focus remains through most of the section, my sense of temporal progression becomes focused to the music on the PA, as it leads me at both the speed of its tempo and the rate of its harmonic change. The conclusion of the piece is thus guided by a kind of musical listening that I have not encountered previously in the piece. This phonograph, for me, encourages a variety of listening experiences, some of which are related to environmental listening, others are related to phonographic listening.

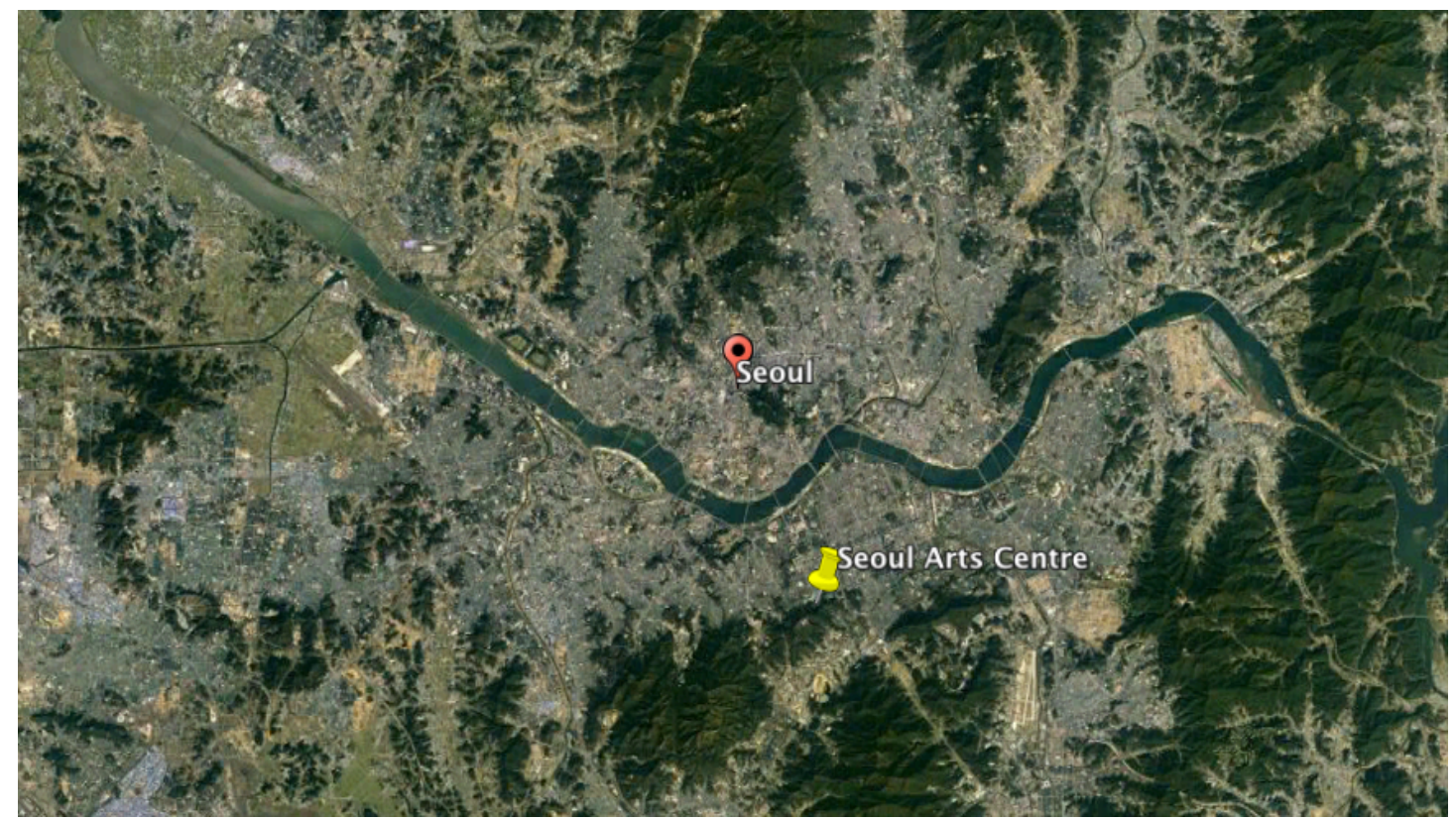

Figure 23: Google, Digital Globe 2016 


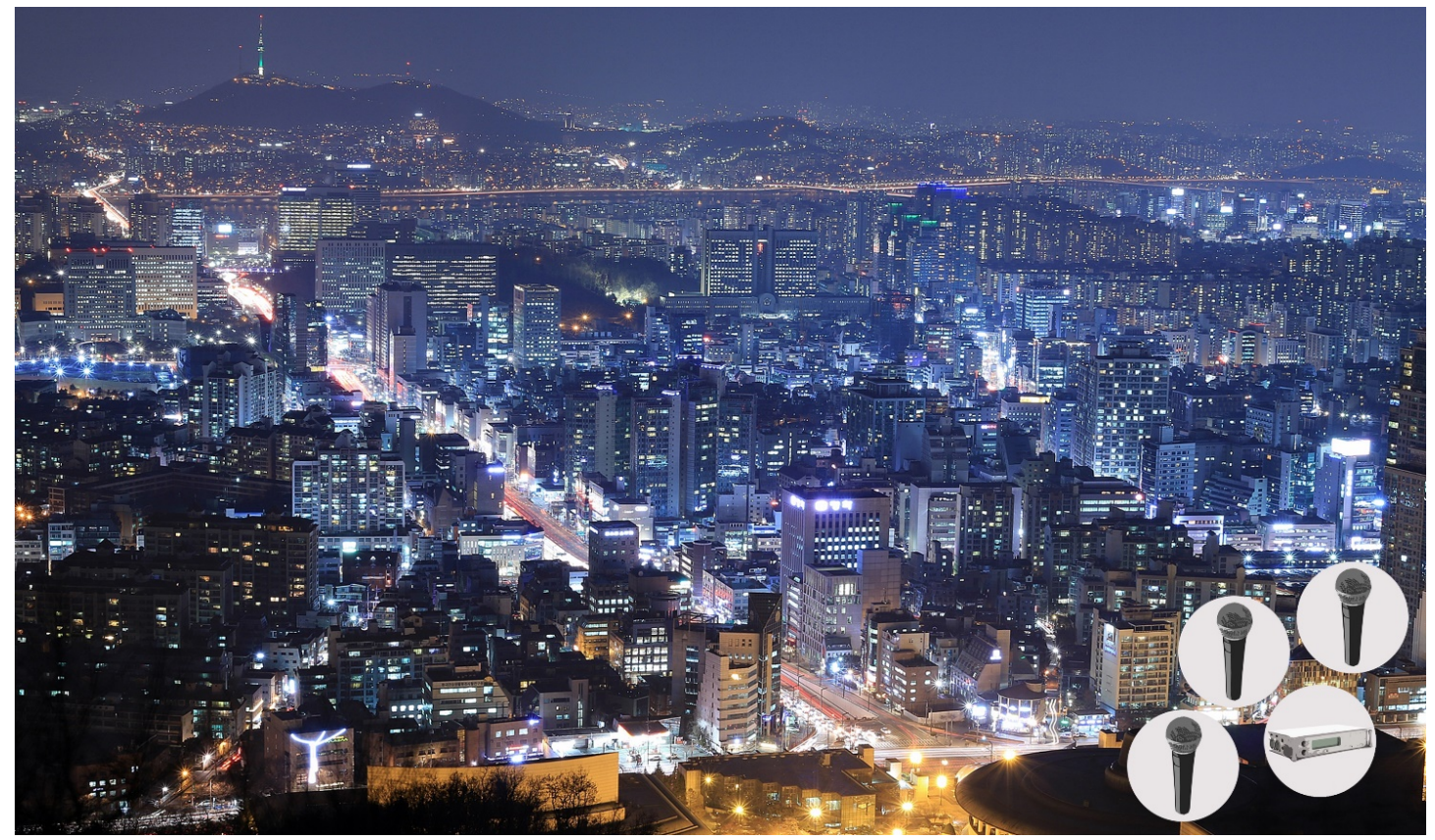

Figure 24: Seoul Art Centre Configuration - Photograph: Donghyun Kim (used with permission); Icons and Layout: Jina Yoon

\section{LIST OF EQUIPMENT USED (IN SITU):}

- Sound Devices 722 recorder

- 2x AKG c451b microphones, set in three different locations behind the Seoul Arts Centre 


\section{Filmography}

Boyens, Phillipa., Jackson, Peter., del Toro, Guillermo and Walsh, Fran. (2013).

"The Desolation of Smaug" [Motion picture]. Directed by Peter Jackson, Wellington: Wingnut Films

Crichton, Michael. (1993).

"Jurassic Park" [Motion picture]. Directed by Steven Spielberg, Los Angeles: Universal Pictures

Herzog, Werner. (2010).

“Cave of Forgotten Dreams" [Motion picture]. Directed by Werner Herzog, Paris: Ministère de la Culture et de la Communication

Johannessen, Bjørn Olaf. (2015).

"Every Thing Will Be Fine" [Motion picture]. Directed by Wim Wenders, Berlin: Neue Road Movies

Lumiere, August and Lumiere, Louis. (1895).

"L'arrivée d'un train en gare de La Ciotat" In "Early Cinema - Primitives and Pioneers" [Motion picture 2005]. Directed by Auguste Lumière and Louis Lumière, London: BFI Video Publishing

Natal, Bruno. (2008).

“Dub Echoes" [Motion picture]. Directed by Bruno Natal, London: Soul Jazz Films

Riedelsheimer, Thomas. (2001).

"Rivers and Tides: Andy Goldsworthy Working with Time" [Motion picture]. Directed by Thomas Riedelsheimer, Berlin: Mediopolis Film und Fernsehproduktion 
Sebestik, Miroslav. (1992).

“Ecoute" [Motion picture 2003]. Directed by Miroslav Sebestik: Paris: JBA Production

Wenders, Wim. (2011).

"Pina" [Motion picture]. Directed by Wim Wenders, Berlin: Neue Road Movies 


\section{Discography}

Barry, J., Greenwich, E and Spector, P. (1963).

“Be My Baby" [Recorded by The Ronettes] On Be My Baby [7" Single]

Philles Records

Bauduc, R., Crosby, B., Haggart, B and Rodin, G.,(2000).

"Swing Set" [Recorded by Jurassic 5] On Quality Control [CD], Interscope

Beck. (2002).

Sea Change [CD], Geffen

Birgisson, A and Wroldsen, I. (2010).

"Impossible" [Recorded by James Arthur] On James Arthur [CD], Simco, (2013)

Body, J. (2007).

The Street Where I Live [Recorded by Jack Body and Stephen de Pledge] On Passing By [CD], Rattle

Brown, H. (1982).

“Ten Dangerous Matches 5" [recorded by Johnny Osbourne] On Scientist Wins the World Cup [LP], Greensleeves Records

Bryant, R and Taylor, J. (1971).

"Fire Eater" [Recorded by Rusty Bryant] On Fire Eater [LP], Prestige

Cage, J. (1953).

Williams Mix [Recorded by John Cage] On Electronic Music Sources Vol. 1 [CD, 2010], Smith and Co 
Crumb, G. (1973).

Black Angels [Recorded by The Kronos Quartet] On Black Angels [CD], Nonesuch, (1990)

Cusack, P. (2012).

Dawn chorus, Chernobyl town [Recorded by Peter Cusack] On Sounds From Dangerous Places [CD], ReR Mega Corp

Donaldson, W. (1926).

"I've Got the Girl" [Recorded by Bing Crosby, Al Rinker, Don Clark and His Los Angeles Biltmore Orchestra] [LP], Columbia

Dubin, A and Warren, H. (1934).

"I Only Have Eyes for You" [Recorded by The Flamingos] On Flamingo Serenade [LP], Reo

Feld, S. (1991).

Voices of the Rainforest [CD], Rykodisk

Ferrari, L. (1970).

Presque Rien No.1/Société II [LP], Deutsche Grammonphon

Getz, S and Gilberto, J (1964).

Getz/Gilberto [LP], Verve Records

Jurassic 5. (2000).

Quality Control [CD], Interscope

Lennon, J and McCartney, P. (1962).

"Love Me Do" [Recorded by The Beatles] On Love Me Do [7" Single], Parlophone 
Lennon, J and McCartney, P. (1967).

"Strawberry Fields Forever" [Recorded by The Beatles] On Strawberry Fields Forever [7" Single], Parlophone

Lennon, J and McCartney, P. (1967).

"I am the Walrus" [Recorded by the Beatles] On Hello Goodbye/I am the Walrus [7" Single], Parlophone

Lennon, J., McCartney, P. and Ono, Y. (1968).

Revolution 9 [Recorded by The Beatles] On The Beatles [LP], Apple Records

Lopez, F. (1998).

La Selva: Sound Environments from a Neotropical Rainforest [CD], Sub Rosa

Lord Finesse. (2014).

The SP1200 Project: A Re-Awakening [CD], Slice of Spice

Miller, J and Swaby, H. (1975).

"Baby I Love You So" [Recorded by Jacob Miller and Augustus Pablo] On Baby I Love You So [7" Single], Yard Music

My Bloody Valentine. (1991).

Loveless [CD], Creation Records

Nirvana. (1989).

Bleach [CD], Sub Pop

Nirvana. (1991).

Nevermind [CD], DGC 
Nirvana. (1993).

In Utero [CD], DGC

Parmegiani, B. (1976).

De Natura Sonorum [LP], INA-GRM

Portishead. (1994).

Dummy [CD], Go! Beat

Public Enemy. (1987).

Yo! Bum Rush the Show [12" LP], Def Jam

Radiohead. (1997).

OK Computer [CD], Parlophone

Ruttmann, W. (1930).

Wochenende [recorded by Walter Ruttmann] On An Anthology of Noise and Electronic Music Vol. 1 [CD, 2006], Sub Rosa

Saddler, J. (1981).

"The Adventures of Grandmaster Flash on the Wheels of Steel" [Recorded by Grandmaster Flash] On The Adventures of Grandmaster Flash on the Wheels of Steel [12" Single], Sugar Hill Records

Schaffer, P. (1948).

Cinq Etudes de Bruits [LP, 2010], Disques Dreyfus

Sharon Jones and the Dap Kings. (2005).

Naturally [CD], Daptone Records

Smashing Pumpkins. (1993).

Siamese Dream [CD], Virgin 
Smith, W. (1985).

"Under Mi Sleng Teng" [Recorded by Wayne Smith and King Jammy] On Under Mi Sleng Teng [7" Single], Jammy's/Greensleeves

Stockhausen, K. (1954).

Studie II [recorded by Karlheinz Stockhausen] On Elektronische Musik 1952-1960 [CD, 1991], Stockhausen-Verlag

The Cinematic Orchestra. (1999).

Motion [CD], Ninja Tune

Ticklah. (2007).

Ticklah vs Axelrod [CD] Easy Star Records

Toop, D (2003)

Silver Birds [Recorded by David Toop] On Black Chamber [CD], Sub Rosa

Truax, B. (1986).

Riverrun [Recorded by Barry Truax] On Digital Soundscapes [CD, 1987] Cambridge Street Records

Westerkamp, H. (1989).

Kits Beach Soundwalk [recorded by Hildegard Westerkamp] On Transformations [CD, 2010], empreintes DIGITALes

Westerkamp, H. (1992).

Beneath the Forest Floor [recorded by Hildegard Westerkamp] On Transformations [CD, 2010], empreintes DIGITALes 


\section{References}

Ashby, A. (2010).

Absolute Music, Mechanical Reproduction. Berkeley: University of California Press

Augoyard, J. F and Torgue, H. (2006).

Sonic Experience: a guide to everyday sounds [trans. Andra McCartney and David Paquette]. Montreal: McGill-Queen's University Press

Azerrad, M. (1993).

Come As You Are: The Story of Nirvana. New York: Doubleday

Azerrad, M. (2001).

Our Band Could Be Your Life. New York: Back Bay Books

Barber, N. (2015, 11 February).

Is James Franco's Latest Movie a Bore? British Broadcasting Corporation. Retrieved from http://www.bbc.com/culture/story/20150211-is-jamesfrancos-latest-a-bore

Barrow, S and Dalton, P. (1997).

Reggae: The Rough Guide. London: Rough Guides

Barthes, R. (1981).

Camera Lucida, [trans. Richard Howard]. New York: Hill and Wang

Barthes, R. (1967).

The Death of the Author [trans. Richard Howard]. Aspen, No. 5-6. New York: Roaring Fork Press. Retrieved from http://www.ubu.com/aspen/aspen5and6/threeEssays.html\#barthes 
Baudrillard, J. (1994).

Simulacra and Simulation [trans. Sheila Glaser]. Michigan: University of Michigan Press

Baumgartner, H. (1992).

Remembrance of Things Past: Autobiographical Memory and Emotion. Advances in Consumer Research, Vol. 19, 613 - 620. Retrieved from http://www.acrwebsite.org/search/view-conferenceproceedings.aspx? $\mathrm{Id}=7363$

Benjamin, W. (2003)

The Work of Art in the Age of its Technical Reproducibility (3 $3^{\text {rd }}$ version). In Eiland and Michael W. Jennings [Eds.] Walter Benjamin: Selected Writings Volume 4, 1938-1940 (p. 251-283). Cambridge: Harvard University Press. (Original work published 1939)

Blesser, B. and Salter L. (2007).

Spaces Speak, Are You Listening?: Experiencing Aural Architecture. Cambridge: MIT Press

Bogost, I. (2012).

Alien Phenomenology or What its Like to be a Thing. Minneapolis: University of Minnesota Press

Boulez, P. (1968).

Notes of an Apprenticeship, [trans by Herbert Weinstock]. New York: Knopf

Bregman, A. S. (1990).

Auditory Scene Analysis: the Perceptual Organization of Sound. Cambridge: The MIT Press 
Buskin, R. (2007, April)

Classic Tracks: The Ronettes 'Be My Baby'. Sound On Sound. Retrieved from

http://www.soundonsound.com/sos/apr07/articles/classictracks_0407. htm

Cage, J. (2004).

The Future of Music: Credo. In C. Cox and D. Warner (Eds.) Audio Culture: Readings in Modern Music (p. 25-28) London: Continuum. (Original work published 1937)

Carlyle, A., \& Lane, C. (2013).

Into the Field: the art of field recording. Devon: Uniform Books

Chion, M. (1994).

Audio-Vision: Sound on Screen. New York: Columbia University Press

Chion, M. (2009).

Guide to Sound Objects: Pierre Schaeffer and Musical Research [trans. John Dack and Christine North). Paris: Institut National De L'audiovisuel \& Éditions Buchet/Chastel

Chua, D. K. L. (1999).

Absolute Music and the Construction of Meaning. Cambridge: Cambridge University Press

Clark, W., \& Cogan, J. (2003).

Temples of Sound: Inside the Great Recording Studios. San Francisco: Chronicle Books 
Coleridge, S. T. (1983).0

Biographia Literaria. In James Engell and Jackson Bate (Eds.). New Jersey: Princeton University Press. (Original work published 1817)

Connolly, K. (2015, 11 February).

Wim Wenders: 3D is like a magnifying glass. The Guardian. Retrieved from http://www.theguardian.com/film/2015/feb/11/wim-wenders-3dberlin-film-festival-every-thing-will-be-fine-james-franco

Connor, S. (2000)

Dumbstruck: A Cultural History of Ventriloquism. Oxford: Oxford University Press

Copeland, D. (2000).

Associative Listening. Soundscape: The Journal of Acoustic Ecology, Vol. 1, No. 1, p. 23-25

Cusack, P. (2000).

Dialogue. Soundscape: The Journal of Acoustic Ecology. Vol. 1, No. 2, p. 8

Cusack, P. (2012).

Sounds From Dangerous Places. Surrey: ReR Megacorp

Dellaira, M. (1995).

Some Recorded Thoughts on Recorded Objects. Perspectives of New Music, 33 (1\&2), p. 192-203

Deleuze, G. (1986)

Cinema 1: The Movement Image [trans. Barbara Habberjam and Hugh Tomlinson]. Minneapolis: University of Minnesota Press 
DPA Microphones

3506A Stereo Kit with 4006A Omnis, (n.d.). Retrieved 12 December, 2014 from

http://www.dpamicrophones.com/en/products.aspx?c=Item\&category= 123\&item $=24512$

DeNora, T. (2000).

Music in Everyday Life. Cambridge: Cambridge University Press

Di Scipio, A. (2013).

Sound Object? Sound Event! Ideologies of Sound and the Biopolitics of

Music. Keynote Address: Musique et écologies du son (Université Paris 8),

Paris. Retrieved from: http://www-artweb.univ-

paris8.fr/spip.php?action=acceder_document\&arg=1386\&cle=4aa3c296e

0ad91b8703cfdd10fb432a0c38c35fc\&file=pdf\%2Fdi_scipio.pdf

Drever, J. L. (2014).

Acoustic Ecology of Great Places. Retrieved 27 November, 2014 from http://www.academyofurbanism.org.uk/acoustic-ecology-of-greatplaces/

Drott, E. (2009).

The Politics of Presque Rien. In R. Adlington (Ed.), Sound Commitments: Avant Garde Music and the Sixties. New York: Oxford University Press

Eco, U. (1989)

The Open Work [trans. Anna Cancogni]. Cambridge: Harvard University Press (originally published in 1962)

Edison, T. (2012)

The Phonograph and its Future. In M. Katz, T. Grajeda \& T.D. Taylor (Eds.), Music Sound and Technology in America (p. 29-37). Durham: Duke University Press. (Original work published 1878) 
Eimert, H. (1957).

What is Electronic Music. Die Reihe, Vol. 1, Theodore Presser Co, p. 1-10

Emmerson, S. (1986).

The Relation of Language to Materials. In S. Emmerson (ed.), The

Language of Electroacoustic Music. London: Macmillan, p. 17-39

Emmerson, S. (2013).

Wandering uneasily in a familiar landscape. eOREMA Journal, No. 1.

Retrieved from http://www.orema.dmu.ac.uk/?q=content/wanderinguneasily-familiar-landscape

Engen, T. (1991)

Odor Sensation and Memory. New York: Praeger Publishers

Eno, B. (2004)

The Studio as a Compositional Tool. In C. Cox and D. Warner (Eds.), Audio Culture: Readings in Modern Music (p. 127-130). London: Continuum

Erlmann, V. (2010).

Reason and Resonance: A History of Modern Aurality. New York: Zone Books

Feld, S. (1994).

Schizophonia to Schismogenesis: On the Discourses and Commodification Practices of "World Music" and "World Beat". In S. Feld and C. Keil (Eds.), Music Grooves. Chicago: Chicago University Press, p. 257-289

Fisher, J. A. (1998).

What the Hills Are Alive With: In Defense of the Sounds of Nature. The Journal of Aesthetics and Art Criticism, Vol. 56, No. 2, p. 167-179 
Goehr, L. (1992).

The Imaginary Museum of Musical Works: An Essay in the Philosophy of Music. Oxford: Oxford University Press

Goldstein, P., \& Rainy, J. (2010, 13 September).

Is Werner Herzog's new 3-D documentary a huge forward leap or total folly? Los Angeles Times. Retrieved from http://latimesblogs.latimes.com/the_big_picture/2010/09/is-wernerherzogs-new-3d-documentary-a-huge-forward-leap-or-total-folly.html

Goldsworthy, A. (2015).

Andy Goldsworthy: Ephemeral Works: 2004-2014. New York: Abrams Books

Goodman, S. (2010).

Sonic Warfare: Sound, Affect, and the Ecology of Fear. Boston: MIT Press

Gould, G. (2004).

The Prospects of Recording. In C. Cox and D. Warner (Eds.), Audio Culture: Readings in Modern Music (p. 115-126). London: Continuum. (Original work published 1966)

Harman, G. (2005).

Guerrilla Metaphysics: Phenomenology and the Carpentry of Things. Peru: Open Court

Henriques, J. (2011)

Sonic Bodies: reggae sound systems, performance techniques, and ways of knowing. London: Continuum 
Hodgkinson, T. (1986, 2 April).

Pierre Schaeffer: an interview with the pioneer of musique concrète.

Interview retrieved 27 March, 2011 from http://www.ele-

mental.org/ele_ment/said\&did/schaeffer_interview.html

Hollerweger, F. (2011).

The Revolution is Hear! (PhD thesis, Queen's University Belfast, Belfast, Northern Ireland)

Homer, M. (2009).

Beyond the Studio: The Impact of Home Recording Technologies on Music Creation and Consumption. Nebula, Vol.6, No. 3 p. 85-99

Hull, J. (2001).

Sound: An Enrichment or State. Soundscape: The Journal of Acoustic Ecology, Vol. 2, No. 1, p. 10-15)

Iges, H. (2000).

Soundscapes: A Historical Approach eContact!, Vol. 3, No. 4 Montréal: CEC.

Ihde, D. (2007).

Listening and Voice: Phenomenologies of Sound (2nd ed.). Albany: State University of New York Press

Ingold, T. (2007)

Against Soundscape. In A. Carlyle (Ed.), Autumn Leaves: Sound and the Environment in Artistic Practice. Paris: Double-Entendre

Jacob, P. (2010).

Intentionality. In E. N. Zalta (Ed.), The Stanford Encyclopedia of Philosophy (Fall 2010 Edition). Retrieved from http://plato.stanford.edu/archives/fall2010/entries/intentionality/ 
Jay, M. (1988).

The Rise of Hermeneutics and the Crisis of Ocularcentrism. Poetics Today, Vol. 9, No. 2, p. 307-326

James, R. (Ed.). (1992).

Cassette Mythos. New York: Autonomedia

Jensen, A. R. (2006).

Clocking the Mind: Mental Chronometry and Individual Differences. Oxford: Elsevier

Kahn, D. (1999).

Noise Water Meat: A History of Sound in the Arts. Massachusetts: MIT Press

Kane, B. (2014).

Sound Unseen: Acousmatic Sound in Theory and Practice. Oxford: Oxford University Press

Kang, J. (2007).

Urban Sound Environment. New York: Taylor \& Francis

Katz, M. (2004).

Capturing Sound: How Technology Has Changed Music. Berkeley: University of California Press

Katz, D. (2003).

Solid Foundation: An Oral History of Reggae. New York: Bloomsbury

Kehew, B., \& Ryan, K. (2006)

Recording the Beatles: The Studio Equipment and Techniques Used To Record Their Classic Albums. Houston: Curvebender Publishing 
Kelman, A. Y. (2010)

"Rethinking the Soundscape: A Critical Genealogy of a Key Term in Sound Studies”. Senses \& Society, Vol. 5, No. 2, p. 212-234

Kock, W. E. (1965)

Sound Waves and Light Waves: The Fundamentals of Wave Motion. New York: Doubleday

Kolber, D. (2002)

Hildegard Westerkamp's Kits Beach Soundwalk: shifting perspectives in real world music. Organised Sound, Vol. 7, No. 1, p. 41-43

La Belle, B. (2006).

Background Noise: Perspectives on Sonic Art. London: Continuum

Landy, L. (1990).

Is more than three decades of computer music reaching the public it deserves? Proceedings: International Computer Music Conference, Glasgow:

p. $369-372$

Leyshon, A. (2009).

The Software Slump: digital music, the democratisation of technology and the decline of the recording studio sector within the musical economy. Environment and Planning A, Vol. 41, No.6, p. 1309 - 1331

Loiperdinger, M. (2004).

Lumiere's Arrival of the Train: Cinema's Founding Myth. The Moving Image, Vol. 4, No. 1, p. 89-118

Lopez, F. (1997).

Schizophonia vs l'objet sonore: soundscapes and artistic freedom. Retrieved from http://www.franciscolopez.net/schizo.html 
Lopez, F. (1998).

Environmental Sound Matter. Retrieved from

http://www.franciscolopez.net/env.html

Maconie, R. (2005).

Other Planets: The Music of Karlheinz Stockhausen. Lanham: Scarecrow Press

Marquardt, S. (n.d.)

Jack Endino Artist Biography. Retrieved 12 September, 2014 from http://www.allmusic.com/artist/jack-endino-mn0000104515/biography

McCartney, A. (2000)

Sounding Places with Hildegard Westerkamp. (PhD thesis, York University, Ontario, Canada)

McIntyre, R., Smith, D. (1989).

Theory of Intentionality. In J. N. Mohanty \& W. R. McKenna (Eds.), Husserl's Phenomenology: A Textbook. Washington: University Press of America

McKinnon, D. (2008).

Spectral Memories: the Aesthetics of the Phonographic Recording. $\mathrm{Hz}$ Journal No. 12. Retrieved from http://www.hzjournal.org/n12/mckinnon.html

Montgomery, W. (2009)

Beyond the Soundscape: Art and Nature in Contemporary Phonography. In J. Saunders (Ed.), The Ashgate Research Companion to Experimental Music. Bath: Ashgate 
Nattiez, J. (1990).

Music and Discourse: Towards a Semiology of Music [trans. Carolyn Abbate]. New Jersey: Princeton University Press

Nickel, Douglas R. (2000).

Roland Barthes and the Snapshot. History of Photography, vol. 24, no. 3, p. $232-235$

Neil, J. (2014, 22 September).

Surround Mixing for Film. CMP0383 lecture, New Zealand School of Music

Ogbar, J. O. G. (2007).

Hip-Hop Revolution: The Culture and Politics of Rap. Kansas: University Press of Kansas

Philipse, H. (1995).

Transcendental Idealism. In B. Smith \& D. W. Smith (Eds.), The Cambridge Companion to Husserl. Cambridge: Cambridge University Press, p. 239323

Pattison, M. (2015, 10 February).

Wim Wenders' 'Every Thing Will Be Fine' is a Major Disappointment. Indiewire. Retrieved from http://www.indiewire.com/article/berlinreview-wim-wenders-every-thing-will-be-fine-is-a-majordisappointment-20150210

Rancière, J. (1991). The Ignorant School Master: Five Lessons in Intellectual Emancipation [trans. Kristin Ross]. Stanford: Stanford University Press

Rancière, J. (2009).

The Emancipated Spectator [trans. Gregory Elliot]. London: Verso 
Rancière, J. (2013).

Notes on the Photographic Image [trans. Darian Meacham]. In N. Mirzoeff (Ed.), The Visual Culture Reader ( $3^{\text {rd }}$ ed.). London: Routledge

Ribowsky, M. (1989).

He's a Rebel: Phil Spector. New York: Cooper Square Press

Romanyshyn, R. D. (1989).

Technology as Symptom and Dream. London: Routledge

Russell, M. (2006).

Husserl: A Guide for the Perplexed. London: Continuum

Russolo, L. (2004).

The Art of Noises: Futurist Manifesto. In C. Cox and D. Warner (Eds.), Audio Culture: Readings in Modern Music (p. 10-14). London: Continuum. (Original work published 1913)

Ruttmann, W. (1930).

Weekend Remix. Retrieved 8 April, 2009 from http://www.medienkunstnetz.de/works/weekend-remix/

Schaeffer, P. (1966).

Traite des objets musicaux. Paris: Èditions du Seuil

Schaeffer, P. (2012).

In Search of a Concrete Music [trans. John Dack and Christine North]. Berkeley: University of California Press

Schafer, R. M. (1977).

The soundscape: our sonic environment and the tuning of the world. New York: Knopf 
Schafer, R. M. (2004).

The Music of the Environment. In C. Cox \& D. Warner (Eds.), Audio Culture: Readings in Modern Music (p. 29-39), London: Continuum. (Original work published 1973)

Schafer, R. M. (2005, May).

Presentation at The Canadian Centre for Architecture, Montreal [transcription by T Voyce]. Audio recording retrieved 7 May, 2009 from http://www.blesser.net/SP_downloads.html

Smalley, D. (1996).

The Listening Imagination: Listening in the Electroacoustic Era. Contemporary Music Review, Vol. 13, Part 2, p. 77-107

Smalley, D. (1997).

Spectromorphology: explaining sound-shapes. Organised Sound Vol. 2, No. 2, p. $107-126$

Smith, S. (2013)

Hip-Hop Turntablism, Creativity and Collaboration. Surrey: Ashgate

Sontag, S. (1977).

On Photography. New York: Penguin Books

Sterling, I. (n.d.).

What is Phonography? Retrieved 14 April, 2008 from http://phonography.org/whatis.htm

Stolzoff, N. C. (2000).

Wake the Town and Tell the People: Dancehall Culture in Jamaica. Durham: Duke University Press 
Stravinsky, I. (2012).

Meine Stellung zur Schallplatte. In M. Katz, T. Grajeda \& T.D. Taylor (Eds.), Music Sound and Technology in America (p. 113). Durham: Duke University Press, (Original work published 1930)

Stravinsky, I. (2012).

An Autobiography. In M. Katz, T. Grajeda \& T.D. Taylor (Eds.), Music Sound and Technology in America (p. 107). Durham: Duke University Press, (Original work published 1936)

Szarkowski, J. (1966).

The Photographer's Eye. New York: Doubleday

Szarkowski, J. (1973).

Looking at Photographs. New York: Bulfinch Press

Tannenbaum, Mya. (1987)

Conversations with Stockhausen [trans. David Butchart]. Oxford: Claredon Press

Taylor, T. D. (1993).

The Gendered Construction of the Musical Self: The Music of Pauline Oliveros. The Musical Quarterly, Vol. 77, No. 3, p. 385-396

Teruggi, D. (2007).

Technology and musique concrète: the technical developments of the Group de Recherches Musicales and their implication in musical composition. Organised Sound, Vol. 12, No. 3, p. 213-231

Toop, D. (1995).

Ocean of Sound: Aether Talk, Ambient Sound and Imaginary Worlds. London: Serpent's Tail 
Toop, D. (2000).

Rap Attack ( $3^{\text {rd }}$ ed.). London: Serpent's Tail

Toop, D. (2004).

Haunted Weather. London: Serpent's Tail

Truax, B. (2001).

Acoustic Communication (2 ${ }^{\text {nd }}$ ed.). Connecticut: Ablex Publishing

Voegelin, S. (2010).

Listening to Noise and Silence. London: Continuum

Voyce, T. (2009).

A Personal and Fragile Affair: the sonic environment and its place in my compositions. (MMus thesis, New Zealand School of Music, Wellington, New Zealand)

Warburton, D. (1998, 22 July).

Luc Ferrari. Interview retrieved 19 December 2008 from http://www.paristransatlantic.com/magazine/interviews/ferrari.html

Watson, A.

Cultural Production in and Beyond the Recording Studio. New York:

Routledge

Weale, R. (2005).

The Intention/Reception Project: Investigating the Relationship between Composer Intention and Listener Response in Electroacoustic Compositions. (PhD Thesis, De Montfort University, Leicester, UK)

Westerkamp, H. (2002).

Linking Soundscape Composition and Acoustic Ecology. Organised Sound Vol. 7 No. 1, p. 51-56 
Westerkamp, H. (1992)

Beneath the Forest Floor (programme notes). Retrieved 21 October 2014 from http://www.sfu.ca/ westerka/program_notes/forestfloor.html

Wrightson, K. (2001)

An Introduction to Acoustic Ecology. Soundscape: The Journal of Acoustic Ecology, Vol. 1, No. 1 p. 10-13

Wyatt, D. (2012, 11 December).

James Arthur's 'Impossible' becomes fastest-selling X Factor single. The Independent. Retrieved from: http://www.independent.co.uk/artsentertainment/tv/news/james-arthurs-impossible-becomesfastestselling-x-factor-single-8406195.html 